\begin{tabular}{|l|l|}
\hline $\begin{array}{l}\text { 2. To: (Receiving Organization) } \\
\text { Distribution }\end{array}$ & $\begin{array}{l}\text { 3. From: (Originating Organization) } \\
\text { Waste Product Technology }\end{array}$ \\
\hline 5. Proj./Prog./Dept./Div.: & 6. Cog. Engr.: \\
TWRS LLW Program & C. N. Wilson (509) 376-1141
\end{tabular}

4. Relațed EDT No.:

7. Purchase order No.:

MMI-SVV-384211 through $-384217$

8. Originator Remarks:

LLW vitrification Phase 1 melter vendor testing offgas and mass balance evaluation report for issuance.

9. Equip./Component No.:

N/A

10. System/Bldg. / Facility:

N $/ A$

11. Receiver Remarks:

$\mathrm{N} / \mathrm{A}$

12. Major Assm. Dwg. No.: N: $/ A$

13. Permit/Permit Application No.: N.A

14. Required Response Date:

N/A

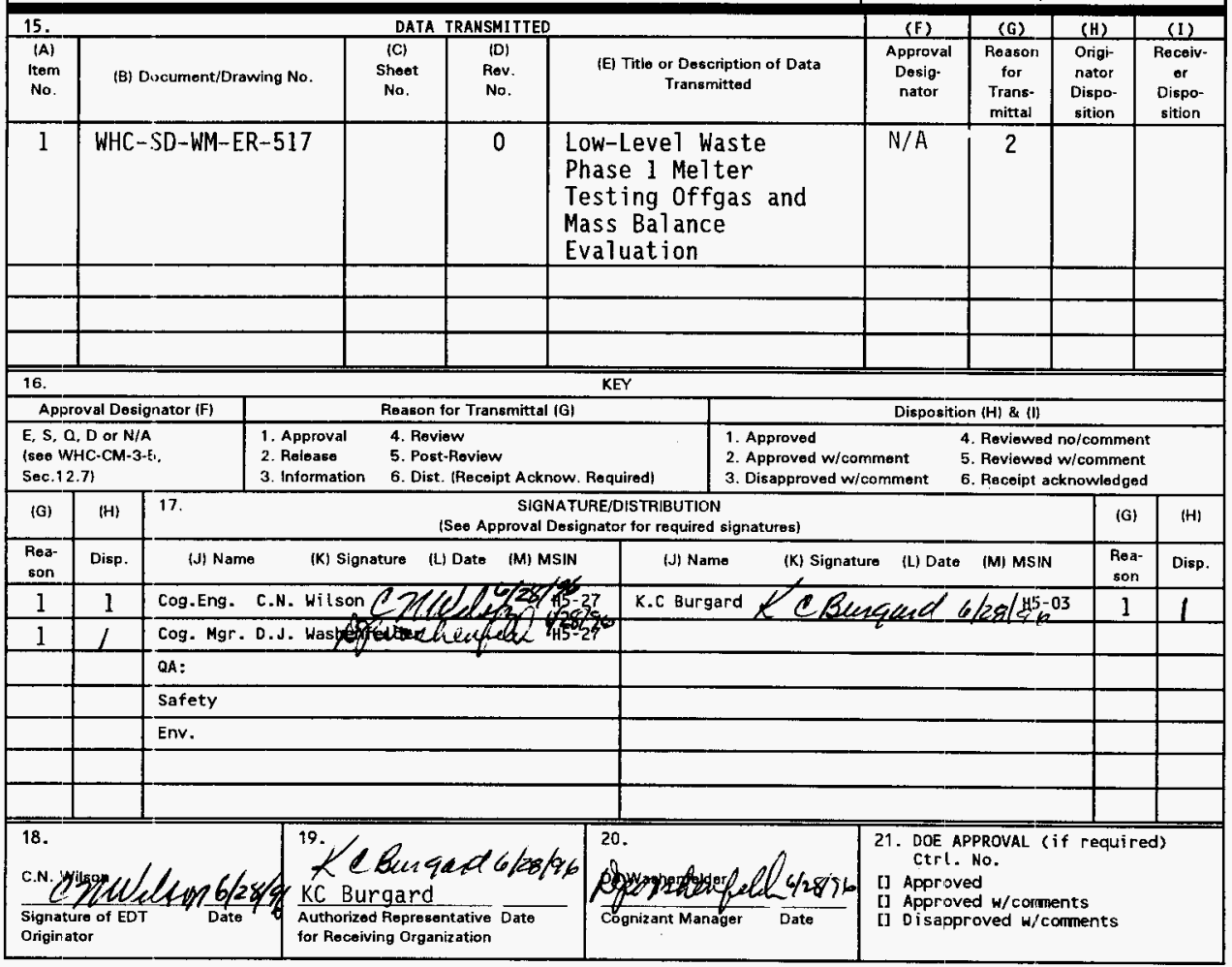




\section{Low-Level Waste Phase 1 Melter Testing Offgas and Mass Balance Evaluation}

C. N. Wilson

Westinghouse Hanford Company, Richland, WA 99352

U.S. Department of Energy Contract DE-AC06-87RL10930

\begin{tabular}{|c|c|c|}
\hline $\begin{array}{l}\text { EDT/ECN: } \\
\text { Org Code: } \\
\text { B\&R Code: }\end{array}$ & $\begin{array}{l}611893 \\
73510 \\
\text { EW3130010 }\end{array}$ & $\begin{array}{l}\text { UC: } 2020 \\
\text { Charge Code: } \\
\text { Total Pages: }\end{array}$ \\
\hline
\end{tabular}

Key Words: Low-level waste, vitrification, melter testing, Hanford Site tank waste, offgas

Abstract: Commercially available melter technologies were tested during 1994-95 as part of a multiphase program to test candidate technologies for vitrification of the low-level waste (LLW) stream to be derived from retrieval and pretreatment of Hanford Site tank wastes. Seven vendors were selected for Phase 1 testing to demonstrate vitrification of a high sodium content liquid LLW simulant. The tested melter technologies included four Joule-heated melters, a carbon electrode melter, a combustion melter, and a plasma melter. Various dry and slurry melter feed preparation processes were also tested. Various feed material samples, product glass samples, and process offgas streams were characterized to provide data for evaluation of process decontamination factors and material mass balances for each vitrification technology. This report describes the melter mass balance evaluations and results for six of the Phase $1 \mathrm{LLW}$ melter vendor demonstration tests.

TRADEMARK DISCLAIMER. Reference herein to any specific comercial product, process, or service by trade name, trademark, manufacturer, or otherwise, does not necessarily constitute or impl.y its endorsement, recommendation, or favoring by the United states Government or any agency thereof or its contractors or subcontractors.

Printed in the United States of America. To obtain copies of this document, contact: WHC/BCS Document Control Services, P.O. Box 1970, Mailstop H6-08, Rich/and WA 92352 , Phone (509) 372-2420; Fax (509) 376-4989.
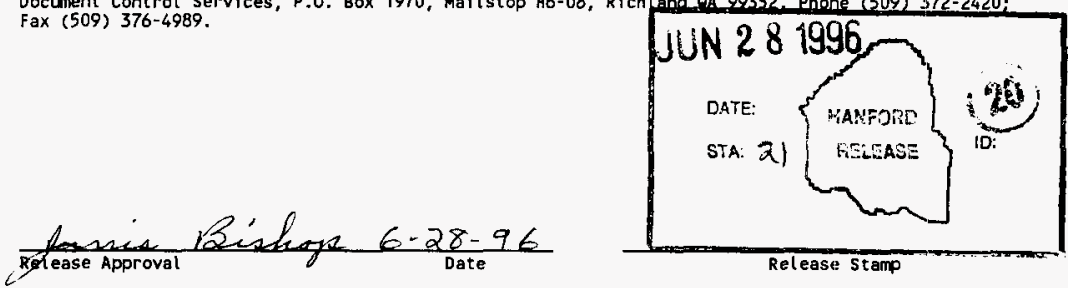

\section{Approved for Public Release}


WHC-SD-WM-ER-517

Revision 0

\section{LOW-LEVEL WASTE PHASE 1 MELTER TESTING OFFGAS AND MASS BALANCE EVALUATION}

G. A. Whyatt and M. R. Powell

Pacific Northwest National Laboratory

G. E. Stegen and C. N. Wilson

Westinghouse Hanford Company

June 1996 
WHC-SD-WM-ER-517

Revision 0

This page intentionally left blank. 
WHC-SD-WM-ER-517

Revision 0

\section{CONTENTS}

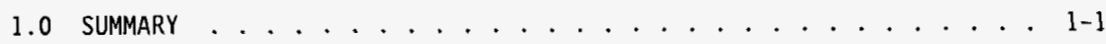

2.0 BACKGROUND . . . . . . . . . . . . . . . . . . . . 2-1

3.0 OFFGAS AND MASS BALANCE EVALUATION . . . . . . . . . . . . . . . . 3-1

3.1 MASS BALANCE EVALUATION METHODOLOGY . . . . . . . . . . . . . . $3-1$

3.2 ESTIMATES OF GROSS ENTRAINMENT OF FEED ............. . . $3-2$

3.2.1 B\&W . . . . . . . . . . . . . . . 3-3

3.2.2 WSTC ...................... 3-3

3.2.3 USBM .................... . . . . . . .

3.2 .4 Vectra... . . . . . . . . . . . . . . . 3-4

3.2 .5 Duratek . . . . . . . . . . . . . . . 3-4

3.2.6 Envitco ................. . . 3-5

3.3 ESTIMATES OF VOLATILE LOSSES OF FEED COMPONENTS . . . . . . . 3-5

3.3.1 B\&W . . . . . . . . . . . . . . . 3-5

3.3.2 WSTC . . . . . . . . . . . . . . .

3.3 .3 USBM ..................... 3-8

3.3.4 Vectra .................. . 3-9

3.3.5 Duratek . . . . . . . . . . . . . . 3-10

3.3.6 Envitco . . . . . . . . . . . . . . . . . 3-11

3.4 TOTAL PARTITIONING TO OFFGAS .................... . . . . . .

3.5 NO EMISSIONS . . . . . . . . . . . . . . . . . . . 3-12

3.5.1 B\&W ..................... 3-12

3.5 .2 WSTC . . . . . . . . . . . . . . 3-13

3.5 .3 USBM . . . . . . . . . . . . . . . . 3-14

3.5.4 Vectra................. . . . . . . . . . .

3.5.5 Duratek . . . . . . . . . . . . . . . . 3-15

3.5 .6 Envitco . . . . . . . . . . . . . . 3-16

3.6 AMMONIA EMISSIONS . . . . . . . . . . . . . . . 3-16

3.6.1 USBM ........................... . . . . . . . . . . .

3.6.2 Vectra................... . . . . . . 16

3.6.3 Duratek . . . . . . . . . . . . . . . 3-16

3.6.4 Envitco ................. . . 3-17

4.0 REFERENCES . . . . . . . . . . . . . . . . . . . . . 4-1

5.0 GLOSSARY . . . . . . . . . . . . . . . . . . . . GL-1 
WHC-SD-WM-ER-517

Revision 0

\section{LIST OF TABLES}

3-1 Percent Gross Entrainment Estimates . . . . . . . . . . . . . 3-3

3-2 Weight Percent Selective Loss of Feed Components . . . . . . . . . 3-6

3-3 Percent of Feed Components Lost to Offgas for Evaluated Melter Systems ..................... . . . . . . . . .

3-4 Percent Yield $\mathrm{NO}_{\mathrm{x}}$ Based on Melter Feed Nitrate and Nitrite Concentrations.................... . 3-14

3-5 Offgas for U.S. Bureau of Mines Type B Feed Reaction and Vectra Feed

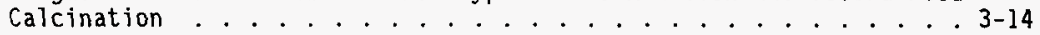


WHC-SD-WM-ER-517

Revision 0

\section{LOW-LEVEL WASTE PHASE 1 MELTER TESTING OFFGAS AND MASS BALANCE EVALUATION}

\subsection{SUMMARY}

A multiphase program was initiated in 1994 to test commercially available melter technologies for vitrification of the low-level waste (LLW) stream to be derived from retrieval and pretreatment of defense wastes stored in underground tanks at the Hanford Site. Testing was conducted at vendor facilities using a nonradioactive chemical simulant representing the composition of double-shell slurry feed tank waste. The double-shel1 slurry feed tank waste is expected to be a major early feed material to an LLW vitrification facility. Seven vendors were contracted for testing and six vendors were successfui in obtaining data. The six melter configurations from which offgas and mass balance data were obtained included a gas-fired cyclone combustion melter, a plasma torch-fired cupola furnace, a carbon electrode melter, two high-temperature molybdenum electrode Joule-heated melters, and a low-temperature Inconel ${ }^{*}$ electrode Joule-heated melter. In addition, each vendor was responsible for the approach to feed preparation before melting. The focus of this report is to review the data and test reports from each run and summarize calculated results for each melter system. The results summarized in this report include the following areas:

- Nonselective loss of feed (assumed to be gross entrainment)

- Selective loss of feed components (assumed to be volatization)

- NO generation from feed nitrates and nitrites.

Gross entrainment was severe $(8.7 \%)$ and significant $(2.7 \%)$ for the combustion melter and the plasma torch furnace, respectively. One Jouleheated melter had very low entrainment $(0.05 \%)$ using a dried feed that was rewet before feeding to reduce dusting. Entrainment in other systems ranged from $0.6 \%$ and $1.2 \%$.

Volatile losses of halogens, alkali metals, boron, molybdenum $\left({ }^{99} \mathrm{Tc}\right.$ surrogate), phosphate, and sulfate were severe for the non-Joule-heated systems. Volatile losses from Joule-heated systems were less severe but still significant. One Joule-heated system obtained low volatile losses using a fu11 coverage dry feed batch blanket. This melter also demonstrated the significant result of only $10 \%$ volatile loss of 1 . The second best melter system for I retention lost $82 \%$ of the I to volatility.

The molar yield of $\mathrm{NO}_{\mathrm{x}}$ to the offgas system (including feed preparation and melter offgas) ranged from $2.6 \%$ to $68 \%$ of the molar $\mathrm{NO}_{3}^{-} / \mathrm{NO}_{2}^{-}$content in the feed. The best performance was obtained using sucrose addition to the feed before slurry feeding of a Joule-heated melter. Other methods demonstrated for $\mathrm{NO}_{x}$ reduction include the addition of powdered sugar and activated carbon to the feed followed by drying and pre-reacting the melter

"Inconel is a trademark of INCO Alloys International, Inc. 


\section{WHC-SD-WM-ER-517}

Revision 0

feeding ( $7.4 \% \mathrm{NO}_{\mathrm{x}}$ yield during feed preparation) and urea addition to the feed before slurry feeding ( $13 \% \mathrm{NO}_{\mathrm{x}}$ yield during melting). Considering the melter unit independently from feed preparation processes, the destruction of residual feed $\mathrm{NO}_{3} / \mathrm{NO}_{2}{ }^{-}$within the carbon electrode furnace resulted in a $\mathrm{NO}_{x}$ yield of only $0.03 \%$. Ammonia emissions were also summarized where data were available. 
WHC-SD-WM-ER-517

Revision 0

\subsection{BACKGROUND}

Following negotiation of the fourth amendment to the Tri-Party Agreement (Ecology et. al. 1994), vitrification became the planned treatment method for the LLW streams derived from retrieval and pretreatment of double-shell tank and single-shell tank wastes at the Hanford Site. A multiphase program was initiated in 1994 to test and evaluate commercially available promising melter technologies using nonradioactive LLW simulants. Seven vendors were selected to participate in Phase 1 testing. A detailed discussion of Phase 1 testing activities and summary results is provided in WHC-SD-WM-ER-498 (Wilson 1996a). The seven Phase 1 vendors and their respective vitrification technologies are as follows.

- Babcock \& Wilcox (B\&W), Alliance Research Center, Alliance, Ohio-Gas-fired cyclone combustion melter, slurry feed

- Envitco, Inc. (Envitco), Toledo, Ohio--High-temperature Joule-heated melter, molybdenum electrodes, wetted pelletized feed

- GTS Duratek Inc. (Duratek), Columbia, Maryland--Low-temperature Joule-heated melter, Inconel electrodes, slurry feed

- Penberthy Electromelt International Inc., Seattle, Washington--Hightemperature Joule-heated melter, sidewall molybdenum electrodes, moist mix-in-the-charger feed

- U.S. Bureau of Mines (USBM), Albany Research Center, Albany Oregon-Carbon electrode melter, prereacted dry feed

- Vectra Technologies Inc. (Vectra), Richland, Washington--Hightemperature Joule-heated melter, top-entry molybdenum electrodes, calcined, dried and slurry feed

- Westinghouse Science and Technology Center (WSTC), Pittsburgh, Pennsylvania--plasma torch-fired cupola furnace, slurry feed.

Phase 1 LLW melter demonstration tests were completed by six of these vendors (testing at Penberthy Electromelt International, Inc. was terminated before completion) from September 1994 through May 1995. A variety of samples were taken during testing by each vendor to address issues defined in WHC-SD-WM-RD-044 (Wilson 1994). Sample types included feed materials, batched melter feed, glass samples, offgas scrub and blowdown solutions, and miscellaneous deposits and residues. Analyses on these samples were performed by contracted independent and government laboratories as follows:

- Quanterra Laboratories, St. Louis, Missouri--Liquid samples including LLW simulant, offgas scrub solutions; and blowdown solutions, chemical analyses, specific gravity, and settled solids 
- Corning Laboratory Services, Corning, New York--Solid samples, feed materials, glass, miscellaneous deposits and residues; chemical analyses, microstructural analyses, phase characterization, and glass redox

- U.S. Geological Survey, Denver, Colorado--Solid samples, feed materials, glass, miscellaneous deposits and residues; chemical analyses, microstructural analyses, phase characterization, and glass redox

- Westinghouse Hanford Company (WHC) 222-S Laboratory--Liquid/slurry samples; specific gravity, and settled and centrifuged solids content

- Pacific Northwest National Laboratory--Radioactive samples (from Duratek DuraMelter-100* [DM-100] test), duplicate analyses of selected samples analyzed at other laboratories, and special and/or expedited analyses.

Results from analyses were sent to WHC for review, distribution, and integration into an LLW melter testing electronic database (Mast. 1995). In addition to these test sample analyses, each vendor contracted with a qualified air quality laboratory to perform specific offgas emissions measurements using standard U.S. Environmental Protection Agency (EPA) methods. These measurements included isokinetic sampling by Methods 5 and/or 29 for multiple metals, Method 26A for determination of hydrogen halides and halogens, and continuous emissions monitoring for $\mathrm{NO}_{x}, \mathrm{CO}_{x}$, and $\mathrm{SO}_{2}$. The air quality laboratory emissions measurement data for each test were reported in each vendor's Phase 1 test reports. These data were reviewed and used as appropriate to perform the presently reported offgas evaluation for each of the six completed Phase 1 vendor LLW melter demonstration tests.

The purpose of this evaluation is to estimate the partitioning of feed components to the offgas system and to estimate the extent to which $\mathrm{NO}_{3}^{-}$and $\mathrm{NO}_{2}{ }^{-}$in the feed are converted to $\mathrm{NO}_{x}$ in the offgas. The partitioning information will provide data needed to design offgas treatment systems. The data will also be used to assess the practicality of offgas recycle and to predict the characteristics of potential recycle streams and secondary waste streams resulting from offgas treatment.

*DuraMelter is a trademark of GIS Duratek, Inc. 


\subsection{OFFGAS AND MASS BALANCE EVALUATION}

A review was conducted of the vendor test reports (Hendrickson 1995 , Eaton 1995a and 1995b, Wilson 1996b, Higley 1995, and Stegen 1996) from each Phase 1 vendor test. The information generated, along with data from the WHC test sample analyses database (Mast 1995), was evaluated to estimate the losses due to selective and non-selective mechanisms, assumed to be volatility and entrainment, respectively. These values are combined to provide the total partitioning of elements to the offgas system. In the case of the Vectra test, where some data from dry feeding are reported, primarily data from the slurry feeding is evaluated because this is Vectra's recommended approach. In the case of Envitco, only the dry feed approach is evaluated due to the absence of offgas data and sample analyses results for the slurry feed test segment. The Duratek evaluation is based on the larger DuraMelter-1000 (DM-1000) melter test.

\subsection{MASS BALANCE EVALUATION METHODOLOGY}

The total loss from the melter is determined from estimates of the entrainment (non-selective loss) and volatility (selective loss). The conceptual model of the process is that the gross entrainment occurs first and equally entrains all components. The volatility then occurs from the remainder. The percent volatilized in this conceptual model can be determined based only on glass and feed composition with no knowledge of the degree of gross entrainment. The equation used to recombine the volatility and entrainment values to determine the fraction of each component partitioning to the offgas is shown below.

$$
L_{T}=L_{e}+L_{v}\left(1-\frac{L_{e}}{100}\right)
$$

where:

$\mathrm{L}_{\mathrm{T}}=$ Percent total loss of component to offgas

$\mathrm{L}_{v}=$ Percent volatile loss of component

$L_{e}=$ Percent non-selective entrainment of feed material.

Entrainment loss values are based primarily on aerosol sampling using EPA-approved Methods 5 or 29 for components assumed to be non-volatile, but may also include data for material that collects in downstream offgas equipment. Calculational methods used for determining volatility include a 'tie component' calculation based on glass and feed composition to determine selective losses, and/or determination of the mass of volatilized components based on offgas measurements. When selective losses are much greater than the probable errors in the glass and feed analyses, the tie component method is generally more reliable than estimates based on offgas measurements. When the 
selective loss is low relative to probable errors in the glass and feed analyses, volatility estimated based on offgas measurements is generally more accurate.

In the tie component method, a component that is known to have little or no volatility at the melter conditions is selected as an internal standard. In addition to being non-volatile, the component should be present in sufficient quantity to allow accurate analytical determination and should not be subject to contamination from non-feed sources such as refractory or electrode corrosion or other sources. Typical tie components selected for these tests include $\mathrm{SiO}_{2}, \mathrm{Al}_{2} \mathrm{O}_{3}, \mathrm{Fe}_{2} \mathrm{O}_{3}, \mathrm{CaO}$, and $\mathrm{SrO}$ with the selection depending on the details of the run. The concentration of the volatile component is compared to the tie component in the feed and glass. The equations are shown below.

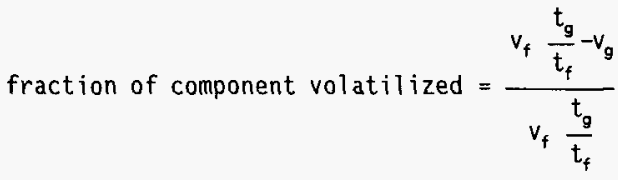

where:

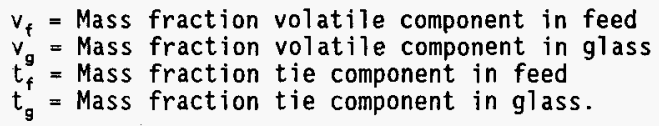

Advantages of the tie component method are that it relies only on compositional analysis and does not require accurate measurement of masses of feed and glass materials. Only the concentration ratios of the components of interest to the concentration of the tie component are important, and the accuracy of the results is not affected by loss of volatile components such as water, nitrates, or carbonates from the feed. In addition, non-selective loss mechanisms such as accumulation, feed spillage, and gross entrainment of the feed, do not affect the results. However, if the melter is not at compositional steady state due to changes in feed composition, errors can be introduced. Also, if there is a source of a component other than from the feed, such as refractory or electrode corrosion, then the selective loss results for the component will smaller than expected or even a riegative value.

\subsection{ESTIMATES OF GROSS ENTRAINMENT OF FEED}

Entrainment refers to material that is physically entrained in the offgas. Entrainment may result from dusting of a dry feed, boiling of wet feed in a cold cap, or a failure of feed to completely segregate from gases for melters such as those at B\&W or WSTC. It is assumed that the loss by entrainment is non-selective and equally entrains all components. Selective 
entrainment is possible, and if present it is included in this evaluation as volatility. Table 3-l provides the best estimates of the rate of entrainment. These values are based primarily on aerosol sampling using EPA-approved Methods 5 or 29, but may also examine material that collects in downstream offgas equipment. Table $3-1$ is organized roughly in the order of decreasing entrainment and volatilization results as observed during the Phase 1 melter vendor testing. In general, the Joule-heated melters show significantly less entrainment than other types of melters.

\subsubsection{B\&W}

The entrainment for the $B \& W$ run was the most severe of any melter system. The entrainment value for B\&W was determined based on Method 5 sample analyses along with feed sample data and using the average of results obtained using $\mathrm{Al}_{2} \mathrm{O}_{3}$ and $\mathrm{CaO}$. There is significant uncertainty in this value due to uncertainty in the feed oxide flow rate and glass production. The value for oxide content of the feed was based on the loss on ignition and loss on drying analytical results $(46.3 \%)$. This value differs significantly from the value used by $\mathrm{B} \& W(56.47 \%)$. Also, the value may be biased low due to losses in the convective pass section before the Method 5 sample point. Additional detail is provided in Appendix A.

\subsubsection{WSTC}

The WSTC entrainment value of $2.7 \%$ is arrived at by taking the difference between the mass of glass poured and the mass of glass expected. It is assumed that the presence of $\mathrm{CaO}$ or $\mathrm{Al}_{2} \mathrm{O}_{3}$ in the offgas samples is due to entrainment only and the mass fraction of $\mathrm{CaO}$ and $\mathrm{Al}_{2} \mathrm{O}_{3}$ in the Method 5 aerosol samples is representative of the composition of the total carryover. This approach results in estimates of $2.1 \%$ and $3.3 \%$ based on $\mathrm{Al}_{2} \mathrm{O}_{3}$ and $\mathrm{CaO}$ content, respectively, which averages to $2.7 \%$. The WSTC test report (Hendrickson 1995) contained a variety of conflicting values for entrainment including $0.2 \%(p .2-10), 0.5 \%$ (p. 8-31), $0.7 \%(p .8-28), 2.0 \%$ (p. $7-18)$, and

Table 3-1. Percent Gross Entrainment Estimates.

\begin{tabular}{rl|c|c|c|c|c|c|c|}
\hline B\&W & WSTC & $\begin{array}{l}\text { USBM } \\
\text { WHCl }\end{array}$ & $\begin{array}{c}\text { USBM } \\
\text { WHC2 }\end{array}$ & $\begin{array}{c}\text { USBM } \\
\text { WHC3 }\end{array}$ & Vectra & Duratek & Envitco \\
\hline 8.7 & 2.7 & 1.2 & N/A & N/A & 0.6 & 0.6 & 0.06 \\
\hline
\end{tabular}


WHC-SD-WM-ER-517

Revision 0

$2.2 \%$ ( $p, 7-14$ ). It is believed that the $2.7 \%$ entrainment value is the best available estimate of gross entrainment. Additional detail is provided in Appendix B.

\subsubsection{USBM}

The USBM conducted three test runs designated WHCl, WHC2, and WHC3. Modifications were made to the melter and operating procedures for runs WHC2 and WHC 3 aimed at reducing volatile component 1 osses. The $1.2 \%$ entrainment estimate for the USBM WHC1 test is based on the mass of $\mathrm{SiO}_{2}$ collected in downstream offgas processing units. An overall mass balance approach suggests a slightly higher value of $1.7 \%$, but the $1.2 \%$ figure is believed to be more accurate. Method 5 samples conducted at the furnace outlet generally indicated lower entrainment values of $0.11 \%$ to $0.47 \%$ based on $\mathrm{Al}$ and $\mathrm{Ca}$ results. However, these results are discounted in favor of the evaluation of silica located in the offgas system due to the difficulties experienced in the Method 5 samples such as plugged sampling lines and nozzles, constriction, and partial plugging at the melter exit which disturbed sampling conditions. Insufficient data were collected during the USBM WHC2 and USBM WHC3 tests to a) low estimation of entrainment losses for these tests. In the absence of other data, it is suggested that the USBM WHCl-based entrainment value be used. However, modifications made to reduce volatile component issses in runs USBM WHC2 and USBM WHC3 also may have resulted in reduced entrainment losses. Additional data are provided in Appendixes $C$ and $D$.

\subsubsection{Vectra}

The value of $0.6 \%$ entrainment for the Vectra MT-2 slurry feed test segment is determined based on Method 5 samples and on the glass and feed compositions to estimate total volatile losses. Total mass loss is estimated at $2.4 \%$ with 0.6 wt\% being by gross entrainment and $1.8 \%$ by volatility. The Method 5 data were sufficiently variable that the 0.6 wt\% entrainment estimate should be considered a fairly rough estimate. Entrainment data also were available from aerosol samples during the MT-3 run segment when the melter was fed dry batched material formulated to simulate a calcined feed composition, and during the MT-4 run segment when fluid bed calcined feed was run. The entrainment during MT-3 was similar to MT-2 giving an entrainment rate of $0.4 \%$ while the entrainment rate during MT-4 was on $1 y 0.07 \%$. Additional detail is provided in Appendix E.

\subsubsection{Duratek}

The Duratek entrainment value of $0.6 \%$ was obtained based on an analysis of Method 5 samples for $\mathrm{Sr}$. The Method 5 sampling data provided by the Vitreous State Laboratory of Catholic University of America (VSL-CUA) were used rather than data from Parsons Engineering Science, Inc. (PES), which was contracted to perform offgas measurements. The PES data were systematically lower which makes the VSL-CUA data more conservative. The more conservative value was selected because the sampling conditions were far from optimum in 
WHC-SD-WM-ER-517

Revision 0

terms of flow conditions and flow quantification. The entrainment value estimate based on the PES data was $0.1 \%$. Additional detail is provided in Appendix F.

\subsubsection{Envitco}

Entrainment calculated for the Envitco dry feed test was very low. The $0.05 \%$ value was calculated based on the amount of Al detected in the Method 29 samples. A comparison also was made to the total amount of material collected on the Method 29 sample. Additional information is provided in Appendix $G$.

\subsection{ESTIMATES OF VOLATILE LOSSES OF FEED COMPONENTS}

Selective loss of components through volatilization is a somewhat greater concern than gross entrainment for the LLW vitrification systems. Volatility estimates were prepared primarily using the previously described tie component method. The tie components typically used were $\mathrm{Al}_{2} \mathrm{O}_{3}, \mathrm{SiO}_{2}, \mathrm{SrO}, \mathrm{CaO}$, and $\mathrm{Fe}_{2} \mathrm{O}_{3}$. In some cases, one or more tie components could not be used because of analytical problems, or because of contamination of the tie component by other sources such as refractory corrosion. In some cases, Method 5 (or 29) offgas sampling results have been used in place of the tie component analysis because the results are believed to be more accurate.

The volatility estimate results are shown in Table 3-2. Shaded values indicate the components of concern for volatility and for which total loss estimates are later provided in Table 3-3. Negative values imply that more material was present in the glass than in the feed material. This may occur because of analytical errors, or from contamination of the glass from refractories or other sources. Component selective loss calculations for each vendor test are contained in Appendixes A through G. A17 I Toss calculations are contained in Appendix H.2.

\subsubsection{B\&W}

In general, the B\&W test showed the greatest volatile component selective losses of the melter systems tested. The volatilities were estimated using the tie component method with $\mathrm{SrO}, \mathrm{SiO}_{2}$, and $\mathrm{CaO}$ as the tie components. The values provided in Table 3-2 are the average of the three tie calculation results. The tie component calculations used analytical data from mass balance test sample sets $1,2,3$, and 5 .

All measurements of $\mathrm{SO}_{3}$ in B\&W glass samples were by USGS and were below detection limits. Corning did not analyze for $\mathrm{SO}_{3}$ in samples from the initial melter tests. Therefore, the estimate provided for $\mathrm{SO}_{3}$ volatility is a

"Mass balance sample sets included simultaneous samples from the melter feed, glass, and offgas streams. 
Table 3-2. Weight Percent Selective Loss of Feed Components. (2 sheets)

\begin{tabular}{|c|c|c|c|c|c|c|c|c|}
\hline Component & $B \& W$ & WSTC & $\begin{array}{l}\text { USBM } \\
\text { WHCI }\end{array}$ & $\begin{array}{l}\text { USBM } \\
\text { WHC2 }\end{array}$ & $\begin{array}{l}\text { USBM } \\
\text { WHC3 }\end{array}$ & Vectra & Duratek & Envitco \\
\hline $\mathrm{Al}_{2} \mathrm{O}_{3}$ & $-36^{8}$ & $.5^{8}$ & $-5.4^{\mathrm{a}}$ & $-3^{\mathrm{a}}$ & 0.49 & $\sim 0$ & -0.1 & $0^{b}$ \\
\hline $\mathrm{B}_{2} \mathrm{O}_{3}$ & 67. & 22 & 51. & 23 & 18. & 14 & $-0^{5}$ & $0.14^{6}$ \\
\hline $\mathrm{BaO}$ & $-51^{c}$ & 5.8 & $12^{c}$ & $-214^{c}$ & $-243^{c}$ & $N / A$ & 14.9 & -61 \\
\hline $\mathrm{CaO}$ & -6.5 & 2.2 & -1.5 & -5.7 & -1.6 & 2.0 & 4.9 & 14 \\
\hline 91. & 87 & 88. & 82. & $N / A$ & 97 . & 64 & 47.8 & $1.10 .13^{6}$ \\
\hline $\mathrm{Cr}_{2} \mathrm{O}_{3}$ & $-76^{\mathrm{B}}$ & 12 & $-32^{a}$ & $-80^{\mathrm{a}}$ & $-64^{a}$ & $-683^{a}$ & $0.6^{\mathrm{b}}$ & $0^{\mathrm{b}}$ \\
\hline $\mathrm{Cs}_{2}{ }^{\circ}$ & 83 & 84 & 63. & N/A & 39 & 41 & 13.2 & $0.6^{6}$ \\
\hline$f^{d}$. & .92 & 91 & 491 & N/A & -99.7 & $-15^{6}$ & -53 & $20.85^{6}$ \\
\hline $\mathrm{Fe}_{2} \mathrm{O}_{3}$ & $-86^{a}$ & -32 & -4.5 & 2.9 & -1.6 & 0.3 & -6.1 & $0^{\mathrm{b}}$ \\
\hline $11:$ & 94 & $>98$ & 95 & W/A & N1/ & 83 & 82 & 10. \\
\hline$k_{2} 0$ & 51 & 48 & 35. & -143 & 25 & 15 & $0^{6}$ & $0^{6}$ \\
\hline 1.10. & N/A & 2.8 & W/A & N/A & W/A & T/A & N/A. & W/1. \\
\hline MgO & $-29^{2}$ & -13 & -25 & -46 & -74 & -2.3 & -12.7 & 2.9 \\
\hline$\overline{\mathrm{MnO}_{2}}$ & $-18^{c}$ & $22^{c}$ & $13^{c}$ & $-686^{c}$ & $-613^{c}$ & $-563^{c}$ & $22.9^{c}$ & $0.13^{\mathrm{b}}$ \\
\hline MoO. & 60 & 24 & 47 & 50 & 45 & -49 & $0.2^{6}$ & $0^{6}$ \\
\hline $\mathrm{la}_{2} \mathrm{O}$ & 35. & 15 & 21. & 20 & 6.3 & 13 & $-0^{6}$ & $0^{6}$. \\
\hline $\mathrm{NiO}$ & $-453^{c}$ & 70 & 73 & $N / A$ & $N / A$ & $N / A$ & -630 & $N / A$ \\
\hline f. & $-41 \%$ & 43 & 41 & -42 & 54 & $1.1^{\circ}$ & $N / A^{\prime}$ & $0^{3}$ \\
\hline S & $>51$ & 34 & 88. & $303^{\circ}$ & 94. & 85 & $\mathrm{~W} / \mathrm{A}$ ' & 53. \\
\hline $\mathrm{SiO}_{2}$ & -0.3 & 11 & 0 & 13 & -0.5 & $\sim 0^{\mathrm{b}}$ & 0 & 0.8 \\
\hline Sro & 10 & -0.1 & 4.0 & -4.2 & $N / A$ & $\sim 0^{\mathrm{b}}$ & $0^{b}$ & $0.10^{\mathrm{b}}$ \\
\hline $\mathrm{TiO}_{2}$ & $-36^{\mathrm{a}}$ & $200^{c}$ & -4.7 & 5.8 & $-104^{c}$ & $\mathrm{~N} / \mathrm{A}$ & 6.6 & $-7^{c}$ \\
\hline $\mathrm{ZrO}_{2}$ & 14 & -0.6 & $-50^{c}$ & $-88^{c}$ & $-621^{c}$ & N/A & 7.5 & $-422^{b}$ \\
\hline
\end{tabular}


Table 3-2. Weight Percent Selective Loss of Feed Components. (2 sheets)

NOTE: Shaded values indicate components likely to be affected by volatility.

Negative values likely due to refractory corrosion increasing component content in the glass.

bvalues based on offgas aerosol data, all other values based on tie component method.

'Low feed concentration such that differences in detection limits between feed and glass, analytical errors, and/or minor contamination results in large errors.

Eifficulty was experienced in analyzing melter feeds for fluoride and feed targets, fluoride concentrations were used to determine all fluoride volatility results. Uncertainty also may exist with respect to fluoride glass analysis. Therefore, all fluoride results are considered approximate. See Appendix I.

Additional detail is provided in text.

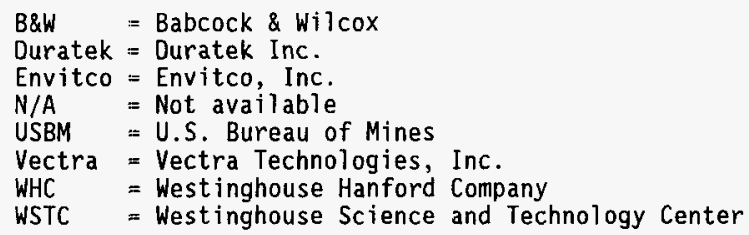

minimum value based on the USGS detection limit in glass. In sample analysis for subsequent melter tests, Corning analyzed for $\mathrm{SO}_{3}$ and achieved much better detection Timits.

The $\mathrm{P}_{2} \mathrm{O}_{5}$ volatility determination was complicated by the feed analysis being consistently below the target values and a source of $\mathrm{P}_{2} \mathrm{O}_{5}$ from refractory corrosion. The result provided is based on assuming the feed contained the target $P$ concentration and on the highest obtained loss result corresponding to a period of lower refractory corrosion.

The $F$ volatility estimate contains significant uncertainty. Analysis for $F$ in feed samples was problematic. Therefore, feed target values for fluoride were used in place of feed analysis data. Corning glass results were systematicaliy lower than USGS results. The USGS results were used to arrive at the given $F$ volatility value of $92 \%$. Volatile losses calculated for $F$ would be higher if the Corning data were used in place of the USlaS data. Additional discussion of the fluoride analytical problems is provided in Appendix I.

The I volatility was taken as the average of four data points where USGS neutron activation analysis results were available for corresponding feed and glass samples. The individual results ranged from an $88 \% 10 s 5$ to a $99 \% 10$ ss of 1 . 
Alumina, $\mathrm{Cr}_{2} \mathrm{O}_{3}, \mathrm{Fe}_{2} \mathrm{O}_{3}, \mathrm{MgO}$ and $\mathrm{TiO}_{2}$ have negative calculated volatilities most likely because the glass content of these components was increased as a result of refractory and metal corrosion. Additional information is provided in Appendix A.

\subsubsection{WSTC}

The WSTC data indicate volatility that is lower than the B\&W system for several key components including $\mathrm{B}_{2} \mathrm{O}_{3}$ and $\mathrm{Na}_{2} \mathrm{O}$. Results were determined based on mass balance sample sets $1,2,3$, and 4 using tie components $\mathrm{Ca}$ and $\mathrm{Al}$. For $\mathrm{SO}_{3}$, the analysis from sample set 3 was discarded due to suspected analytical error to arrive at the value provided. The measured glass $\mathrm{Fe}$ content is well within detection limits and indicates an unidentified source of Fe contamination exists increasing the glass content relative to the feed. The reason for the indicated $11 \% \mathrm{SiO}_{2}$ volatility is uncertain. It is not expected that $\mathrm{SiO}_{2}$ has any significant volatility. This may be the result of the unsteady mix ratio between waste simulant and glass formers. However, analytical or sampling errors and contamination of the glass with additional $\mathrm{Al}$ and $\mathrm{Ca}$ as a result of refractory corrosion also may have contributed to the calculated $\mathrm{SiO}_{2}$ volatility. Additional information is provided in Appendix $B$.

Iodine volatility was determined based on neutron activation analysis by USGS of one feed sample and one glass sample. The I in the glass sample was below detection limits and the value provided was calculated using the analytical 'less than' result.

\subsubsection{USBM}

Data from the initial USBM WHCl test indicate very significant volatility of key components such as $\mathrm{B}_{2} \mathrm{O}_{3}(51 \%), \mathrm{Na}_{2} \mathrm{O}(21 \%)$ and $\mathrm{MoO}_{3}$ (47\%). However, the volatility evaluation was complicated from contamination from 'Tree Hugger'* feed material used at the beginning of the USBM WHCl run. Results were based on an $\mathrm{SiO}_{2}$ tie component using average feed and glass concentrations after correcting $\mathrm{SiO}_{2}, \mathrm{CaO}, \mathrm{Al}_{2} \mathrm{O}_{3}$, and $\mathrm{TiO}_{2}$ for the apparent contamination. The identicaliy zero result for the $\mathrm{SiO}_{2}$ is a direct result of the selection of $\mathrm{SiO}_{2}$ as a tie component. Negative volatilities calculated for some components may be related to the addition of Tree Hugger feed components to the glass. Additional information for the USBM WHCl run is provided in Appendix C.

Calculation of selective component losses by the tie component method for the USBM tests also was complicated by the occasional addition of 1 ime to the melter as a fluxing agent when the glass became too viscous to drain, and by contamination from steel punchings used for starting the furnace in later runs. The volatilities were obtained by first adjusting the glass composition to remove excess $\mathrm{Ca}$ and $\mathrm{Fe}$ based on the silica and alumina concentrations. The results were taken as the mid-range value of the calculated results using feed analysis and feed targets with $\mathrm{SiO}_{2}$ and $\mathrm{Al}_{2} \mathrm{O}_{3}$ tie components.

*Feed material previously melted by USBM for the Tree Hugger Corporation is defined in WHC-SD-WM-VI-030, p. 3-4 (Eaton 1995a). 
The $95 \%$ I volatility result was determined using USGS neutron activation analysis results for six samples of dried and reacted feed and seven glass samples. The feed samples were used to estimate an average feed composition and loss estimates were made based on each glass sample. Loss estimates ranged from $91 \%$ to $99 \%$.

As a result of the high component volatilities observed in the USBM WHCI run, modifications were made to the melter and in the operating conditions intended to reduce volatility. The USBM reduced the voltage and power input for run USBM WHC2, and based on favorable indications, further decreased the power density on the electrodes for USBM WHC3 by increasing the electrode diameter. Significantly lower volatilities for $\mathrm{Na}_{2} \mathrm{O}, \mathrm{Cs}_{2} \mathrm{O}$, and $\mathrm{B}_{3} \mathrm{O}_{3}$ were achieved during these later runs." Selective loss calculations for USBM runs WHC2 and WHC3 are provided in Appendix 0.

The fluoride loss is based on feed target values rather than analytical feed values. However, because of the large loss, the impact of this assumption is only to increase the fluoride loss to $99.7 \%$ compared to $99.5 \%$ if feed analys is values are used.

Interestingly, while the losses of alkali metals and B were significantly reduced in the USBM WHC3 run, the volatile losses of halogens are consistently greater in the revised operating conditions. The trend is believed to be real although the mechanistic explanation is unknown. Activation analysis data were unavailable to estimate I losses for the USBM WHC2 and USBM WHC3 runs.

\subsubsection{Vectra}

The Vectra results indicated volatility somewhat similar to the USBM- 3 except that halogen losses were much lower. All results in Table 3-2 are evaluated for the slurry feeding method (run MT-2) which was the recommended feed method. Values for $\mathrm{Cs}_{2} \mathrm{O}, \mathrm{K}_{2} \mathrm{O}, \mathrm{F}, \mathrm{Cl}, \mathrm{I}, \mathrm{SO}_{3}$, and $\mathrm{P}_{2} \mathrm{O}_{5}$ were obtained based on a Sro tie component. This was done because these minor components iplus Sro) were not in the startup glass and may not have reached steady-state concentrations by the time the slurry feed MT-2 run mass balance period was started. The remaining results are the average of results obtained using $\mathrm{CaO}$, $\mathrm{Al}_{2} \mathrm{O}_{3}, \mathrm{Fe}_{2} \mathrm{O}_{3}$, and $\mathrm{SiO}_{2}$ as the tie components.

The results obtained based on Method 5 offgas analyses are adjusted assuming that gross entrainment is $0.6 \%$. In cases where the Method 5 initial result was $<0.6 \%$, the result is expressed as $\sim 0$. Aerosol sampling results are believed to be biased low due to line losses between the melter and the sample point. However, for some analytes, the aerosol samples provide improved data over the tie component result. The fluoride result was taken from aerosol sample data due to analytical difficulties experienced in the glass for fluoride. The $\mathrm{P}_{2} \mathrm{O}_{5}$ data were taken from aerosol samples due to uncertainty about the actual feed $P$ concentrations. The tie component calculation indicates an excess of $\mathrm{P}_{2} \mathrm{O}_{5}$ in the glass compared to feed target values. The WHC3.

"Based on glass sample analyses performed by USBM for runs in WHC2 and 
single feed sample that was analyzed for $P$ indicated an excess of $P_{2} O_{5}$ in the feed. The aerosol-based result is less sensitive to the feed concentration uncertainty. There was a significant discrepancy between the aerosol result and the tie component result for $\mathrm{Na}_{2} \mathrm{O}$. The aerosol result was only $4 \% \mathrm{Na}_{2} \mathrm{O}$ loss compared to the $13 \%$ loss by the tie component result. It was believed that for $>10 \%$ loss of a major component the tie component calculation was more reliable. However, there is a possibility that the volatility was lower and that the result was related to errors in the feed composition analyses.

The tie component $\mathrm{Cr}_{2} \mathrm{O}_{3}$ result was $-683 \%$ which reflects corrosion of refractory and the stainless steel electrode sheaths. Similarly, the tie component analysis result for $\mathrm{MoO}_{3}$ is $-49 \%$ which reflects the loss of electrode material. The Method 5 results for $\mathrm{Cr}_{2} \mathrm{O}_{3}$ and $\mathrm{MoO}_{3}$ indicate that $5.6 \%$ and $115 \%$ of the $\mathrm{Cr}$ and Mo contained in the feed, respectively, were volatilized to the offgas (corrected for $0.6 \mathrm{wt} \%$ entrainment).

The $83 \%$ volatility result for $I$ is based on neutron activation analysis of four glass samples by USGS using a Sro tie component calculation. Suitable feed samples were unavilable so the feed target values were used for feed I content.

In addition to the slurry feeding data, Vectra generated data from feeding of dry batched materials with a composition intended to match a calcined feed (Run MT-3) and from running actual calcined feed (Run MT-4) . Because of the short durations, tie component calculations were not possible for these testing periods. However, aerosol sample data were taken and can be used to estimate volatile losses. During Run MT-3, it appeared that the volatility was lower than for slurry feeding. During Run MT-4, it appeared that the volatility was as high or higher than slurry feeding and it appeared that significant unidentified sources of chloride and $S$ were present.

Additional information is provided in Appendix $\mathrm{E}$.

\subsubsection{Duratek}

The Duratek DM-1000 melter experienced only moderate volatility losses. Results provided in Table 3-2 are, with a few exceptions discussed below, obtained using USGS analyses results for feed and glass and using $\mathrm{SiO}_{2}$ as a tie component. Corning analytical results for feed were not used because no analysis was available for mixed feed and the simulant analys is indicated a significant deficiency in $\mathrm{Al}_{2} \mathrm{O}_{3}$ which may have been due to either sampling or analytical error. In the case of $\mathrm{MgO}$, the Corning analytical results were selected because USGS failed to obtain a result greater than detection limits. For the fluoride result, the target feed concentration was used in place of the analytical value due to the analytical problems that were generally experienced for this analyte. Additional information is provided in Appendix F.

The selective loss values obtained using aerosol results used the VSL-CUA Method 5 data given in Table 5.7 e of WHC-SD-WM-VI-027 (Eaton 1995b) and USGS feed analyses data, and were obtained by adjusting the data to remove the contribution from gross entrainment calculated assuming the $\mathrm{Sr}$ content in the Method 5 samples was due entirely to entrainment. Where the loss of a 
component was less than or equal to the Sr loss, a value of 0 is given. The VSL-CUA Method 5 results were chosen over the PES data because they were systematically higher values and provided more conservative results. This conservatism is considered warranted due to inadequate offgas flow measurement, the difficult sampling configuration, and the lack of agreement between the VSL-CUA and PES data.

A selective loss value of $-27 \%$ for $\mathrm{P}_{2} \mathrm{O}_{5}$ was obtained based on tie component calculations. The $\mathrm{P}_{2} \mathrm{O}_{5}$ content for the Duratek target glass was 0.19 wt\%. When Duratek performed laboratory-scale test melts before operation, the $\mathrm{P}_{2} \mathrm{O}_{5}$ content of the glasses was $0.32 \mathrm{wt} \%$. This result implies a $-68 \%$ volatility result for the laboratory melt when compared to feed analysis. This would tend to indicate that the feed contained too much $\mathrm{P}_{2} \mathrm{O}_{5}$. By examining the data for the $\mathrm{P}_{2} \mathrm{O}_{5}$ in the glass, it is apparent that the glass concentration, and to a lesser extent the feed concentration, is dropping over time. The ratio of $\mathrm{P}_{2} \mathrm{O}_{5}$ to $\mathrm{SiO}_{2}$ calculated for sequential glass sampling times is $7.1,7.0,6.6,6.7$, and 5.8 (all $\times 10^{-3}$ ); the sequential $\mathrm{P}_{2} \mathrm{O}_{5}$ to $\mathrm{SiO}_{2}$ ratios in the feed were $4.93,4.82$, and $3.81\left(\mathrm{a} 11 \times 10^{-3}\right)$. It is possible that there was phosphorus contamination in the system, and because the glass $\mathrm{P}_{2} \mathrm{O}_{5}$ to $\mathrm{SiO}_{2}$ ratio was lagging the feed concentration, the data indicate a volatility gain across the melter with time. Because there are no Method 5 data on which to base an alternate $\mathrm{P}_{2} \mathrm{O}_{5}$ volatility estimate, it does not appear that there is sufficient information on which to base a defensible $P_{2} \mathrm{O}_{5}$ volatility loss estimate.

A special estimate for $\mathrm{SO}_{3}$ volatility was prepared based on an average of the USGS sample analys is data providing information on $\mathrm{SO}_{3}$ (three feed, two glass) and using $\mathrm{SiO}_{2}$ as a tie component. The result indicates $\mathrm{SO}_{3}$ volatility of $7.2 \%$ with sample standard deviation of $\pm 17.3 \%$ based on analysis of the USGS sample variability. Analytical data consistently indicate that feed contained more $S$ than the feed target composition. The offgas $\mathrm{SO}_{2}$ data at the highefficiency particular air filter exit are inconsistent with feed data and indicate substantially more $\mathrm{SO}_{2}$ in the offgas than was fed to the melter.

The $82 \%$ volatility result for I was determined based on neutron activation analysis of three feed samples and two glass samples using an $\mathrm{SiO}_{2}$ tie component calculation.

\subsubsection{Envitco}

The Envitco dry feed test had the lowest selective component volatility losses of any test. Results were determined based on an average of the results obtained using $\mathrm{SiO}_{2}, \mathrm{SrO}$, and $\mathrm{Al}_{2} \mathrm{O}_{3}$ tie components. With the exception of $S$ and $I$, losses were too low to accurately determine the volatility using the tie component calculation. However, the results of the tie component calculation confirm that the volatile losses were low. Results obtained based on Method 29 sample data assume that the gross entrainment was $0.05 \%$ and have been corrected to account for entrainment. The volatile loss calculations for $\mathrm{Cl}$ and $\mathrm{F}$ were hindered by poor detection limits on the samples and are given as ranges. 
The $\mathrm{SO}_{3}$ selective loss value of $53 \%$ was based on only one glass sample for which detectable $\mathrm{SO}_{3}$ was reported. Based on a second glass sample for which a less than value was reported, the $\mathrm{SO}_{3}$ volatility loss would be $>34 \%$. offgas $\mathrm{SO}_{2}$ monitoring, which indicated $\mathrm{SO}_{2}$ emissions accounted for $37 \%$ of the feed $S$ content, also indicated significant $S$ volatility.

The I result of $10 \%$ volatility was determined using USGS neutron activation analysis data for one feed sample and three glass samples. The feed sample appeared to be slightly deficient in I and if the target feed composition is used, the volatility is calculated at $16 \%$ with a maximum individual result of $24 \%$. However, regardless of the treatment of the data the qualitative conclusion remains that the melter retained a significant fraction of the I within the glass.

\subsection{TOTAL PARTITIONING TO OFFGAS}

Total partitioning of volatile components to the offgas system obtained by combining the non-selective losses (assumed to be entrainment) in Table 3-1 with the selective losses (assumed to be volatility) in Table 3-2, using the methodology described in section 3.1, are provided in Table 3-3. Supporting calculations are provided in Appendix H.3.

\section{$3.5 \mathrm{NO}_{x}$ EMISSIONS}

The generation of $\mathrm{NO}_{\mathrm{x}}$ was examined for each of the vendor technologies: An $\mathrm{NO}_{x}$ yield was calculated by comparing the molar feed rate of $\mathrm{NO}_{3}^{-}$and $\mathrm{NO}_{2}$ : to the molar emission rate of $\mathrm{NO}_{x}$ in the offgas. No attempt was made to separate $\mathrm{NO}_{x}$ sources between thermal $\mathrm{NO}_{x}$ resulting from $\mathrm{N}_{2}$ in air and $\mathrm{NO}_{\mathrm{x}}$ resulting from feed $N$. A $100 \%$ yield was assumed to resuft in 1 mol of No for each mole of $\mathrm{NO}_{3}^{-}$and $\mathrm{NO}_{2}^{-}$in the feed to the melter. Where appropriate, $\mathrm{NO}_{x}$ yields were calculated separately for the feed preparation processes and for the actual melter unit itself. A summary of the $\mathrm{NO}_{\mathrm{x}}$ yields considering the melter unit only is provided in Table 3-4 followed by a brief description of the calculation used to arrive at the value for each vendor. Supporting calculations for $\mathrm{NO}_{\mathrm{x}}$ yields during feed preparation and melter operations are provided in Appendix H.1.

The $\mathrm{NO}_{\mathrm{x}}$ yields during preparation of the USBM Type B feed and Vectra fluid bed calcined feed are given in Table 3-5. The USBM data are from a sample of damp USBM Type B feed taken from the ribbon blender during feed production and was tested by PNNL to measure the gases released during the drying and reaction process. The Vectra calcination offgas data were measured during feed calcination by Procedyne and reported in WHC-SD-WM-VI-031, Part 1 (Stegen 1996).

\subsubsection{B\&W}

The NO yield of $68 \%$ for B\&W applies to operation without feed additives to reduce $\mathrm{NO}_{3}^{-} / \mathrm{NO}_{2}^{-}$in the feed and with neither reburn or selective noncatalytic reduction operating. The relatively high yield may be due in part 
Table 3-3. Percent of Feed Components Lost to Offgas for Evaluated Melter Systems.

\begin{tabular}{|l|l|l|l|l|l|l|l|l|}
\hline Component & B\&W & WSTC & $\begin{array}{c}\text { USBM } \\
\text { WHC1 }\end{array}$ & $\begin{array}{l}\text { USBM } \\
\text { WHC2 }\end{array}$ & $\begin{array}{l}\text { USBM } \\
\text { WHC3 }\end{array}$ & Vectra & Duratek & Envitco \\
\hline $\mathrm{B}_{2} \mathrm{O}_{3}$ & 70 & 24 & 52 & 24 & 19 & 15 & 0.6 & 0.2 \\
\hline $\mathrm{C} 1$ & 88 & 88 & 82 & N/A & 97 & 64 & 48 & 1 to 13 \\
\hline $\mathrm{Cs}_{2} \mathrm{O}$ & 85 & 84 & 63 & N/A & 40 & 41 & 14 & 0.6 \\
\hline $\mathrm{F}^{*}$ & 93 & 91 & 91 & N/A & 99.7 & 16 & 53 & 0.90 \\
\hline $\mathrm{I}$ & 95 & 98 & 95 & N/A & N/A & 83 & 82 & 10 \\
\hline $\mathrm{K}_{2} \mathrm{O}$ & 55 & 49 & 36 & SE & 26 & 16 & 0.6 & 0.05 \\
\hline $\mathrm{Li}_{2} \mathrm{O}$ & $\mathrm{N} / \mathrm{A}$ & 5.4 & $\mathrm{~N} / \mathrm{A}$ & $\mathrm{N} / \mathrm{A}$ & $\mathrm{N} / \mathrm{A}$ & $\mathrm{N} / \mathrm{A}$ & $\mathrm{N} / \mathrm{A}$ & $\mathrm{N} / \mathrm{A}$ \\
\hline $\mathrm{MoO}_{3}$ & 64 & 26 & 48 & 51 & 46 & $\mathrm{SE}$ & 0.8 & 0.05 \\
\hline $\mathrm{Na}_{2} \mathrm{O}$ & 41 & 17 & 22 & 21 & 7.6 & 13.5 & 0.6 & 0.05 \\
\hline $\mathrm{P}$ & 46 & 45 & 42 & SE & 55 & 1.7 & SE & 0.05 \\
\hline $\mathrm{S}$ & 55 & 36 & 88 & SE & 94 & 85 & SE & 53 \\
\hline
\end{tabular}

"Analytical difficulties experienced with fluoride analysis make all values approximate. See Appendix I.

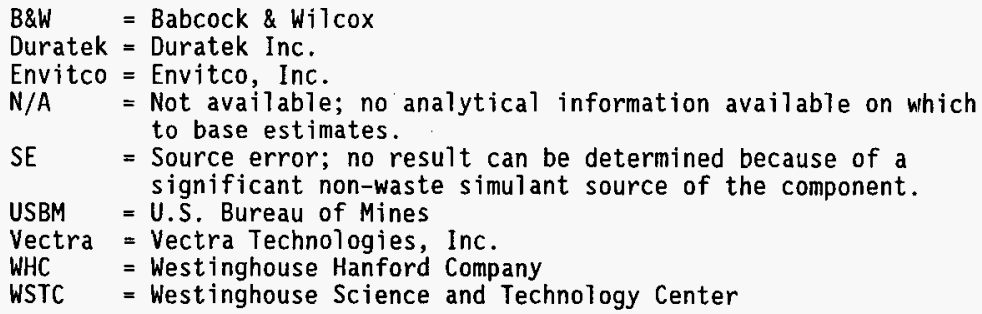

to a relatively high offgas flow rate and generation of thermal $\mathrm{NO}_{\mathrm{x}}$. A substantial fraction of the $\mathrm{NO}_{x}$ measured may be thermal $\mathrm{NO}_{\mathrm{x}}$ and not originate from feed $\mathrm{N}$.

\subsubsection{WSTC}

Additives were not used to reduce $\mathrm{NO}_{3}^{-} / \mathrm{NO}_{2}^{-}$in the WSTC feed. The $\mathrm{NO}_{\mathrm{X}}$ yield for the WSTC plasma torch was calculated as $8 \%$ based on online monitor data. The $\mathrm{NO}_{\mathrm{x}}$ is attributed to thermal sources and no change in the online reading was observed between feeding and non-feeding operating conditions. Bomb samples indicated $\mathrm{NO}_{\mathrm{x}}$ levels 5 to 20 times higher than the online 
Table 3-4. Percent Yield NO ${ }_{x}$ Based on Melter Feed Nitrate and Nitrite Concentrations.

\begin{tabular}{|c|c|c|c|c|c|c|c|}
\hline B\&W & WSTC & $\begin{array}{l}\text { USBM } \\
\text { WHC1 }\end{array}$ & $\begin{array}{l}\text { USBM } \\
\text { WHC2 }\end{array}$ & $\begin{array}{l}\text { USBM } \\
\text { WHC3 }\end{array}$ & Vectra & Duratek & Envitco \\
\hline 68 & $\sim 8$ & $0.03^{*}$ & N/A & N/A & 2.6 & 13 & $\sim 33^{\text {a }}$ \\
\hline
\end{tabular}

"Prereacted feed. Does not include $\mathrm{NO}_{\mathrm{x}}$ generation in feed

preparation. Values including the feed preparation process emissions are USBM $W H C l=7.4 \%$, Envitco $=25 \%$ to $50 \%$.

$\begin{array}{ll}\text { B\&W } & =\text { Babcock \& Wilcox } \\ \text { Duratek } & =\text { GTS Duratek, Inc. } \\ \text { Envitco } & =\text { Envitco, Inc. } \\ \text { USBM } & =\text { U.S. Bureau of Mines } \\ \text { Vectra } & \text { Vectra Technologies, Inc. } \\ \text { WHC } & =\text { Westinghouse Hanford Company } \\ \text { WSTC } & =\text { Westinghouse Science and Technology Center }\end{array}$

Table 3-5. Offgas for U.S. Bureau of Mines Type B Feed Reaction and Vectra Feed Calcination.

\begin{tabular}{|l|c|c|c|}
\hline \multirow{2}{*}{$\begin{array}{l}\text { Gaseous } \\
\text { species }\end{array}$} & \multicolumn{2}{|c|}{$\begin{array}{c}\text { U.S. Bureau of Mines } \\
\text { Type B feed } \\
\text { laboratory data }\end{array}$} & $\begin{array}{c}\text { Vectra Technologies, } \\
\text { Inc. fluid bed } \\
\text { calcination }\end{array}$ \\
\cline { 2 - 4 } & $\begin{array}{c}\text { mmol } \\
\text { release }\end{array}$ & $\begin{array}{c}\text { \% of gaseous } \mathrm{N} \\
\text { released as }\end{array}$ & $\begin{array}{c}\text { \% of gaseous } \mathrm{N} \\
\text { released as }\end{array}$ \\
\hline $\mathrm{NO}_{\mathrm{x}}$ & 23 & 9.6 & 3.7 \\
\hline $\mathrm{N}_{2} \mathrm{O}$ & 18.8 & 15.6 & 4.7 \\
\hline $\mathrm{NH}_{3}$ & 23.5 & 9.8 & 0.4 \\
\hline $\mathrm{N}_{2}$ & 78.2 & 65 & 91 \\
\hline
\end{tabular}

instruments. The average yield would be approximately $62 \%$ if the results of bomb samples were used. The results for Ar from the bomb samples were uncertain and WSTC believed that the bomb samples were more likely the incorrect analys is for $\mathrm{NO}_{x}$. However, there was significant potential to lose some $\mathrm{NO}_{\mathrm{x}}$ due to condensation in sample lines and subsequent scrubbing of $\mathrm{NO}_{\mathrm{x}}$. Therefore, a value higher than $\sim 8 \%$ yield may be justified depending on the use of the data.

\subsubsection{USBN}

In the USBM feed preparation process, powdered sugar and activated carbon were added to the feed and the mixture was heated to dry the feed and react 
the nitrates before feeding the melter. The feed preparation process removed $77.5 \%$ of the $\mathrm{NO}_{3}{ }^{-} / \mathrm{NO}_{2}{ }^{-}$from the feed. Offgas data were unavailable from the feed preparation process. However, a sample of the unreacted feed was reacted in the laboratory (at PNNL) and the gases evolved were measured and are shown in Table 3-5. It is estimated that of the nitrogen released as gas in the feed preparation reaction, $9.6 \%$ of it is released as $\mathrm{NO}_{\mathrm{x}}$.

The calculated efficiency based on the $\mathrm{NO}_{3}{ }^{-} / \mathrm{NO}_{2}^{-}$destruction within the melter itself is extremely high $(99.97 \%)$. This vafue is based on the remaining residue of $\mathrm{NO}_{3}^{-} / \mathrm{NO}_{2}{ }^{-}$within the feed and the $\mathrm{NO}$, data resulting from monitoring the offgas. Thus, the majority of the $\mathrm{NO}_{\mathrm{x}}$ was evolved during the feed preparation process while the amount of $\mathrm{NO}_{\mathrm{x}}$ generated within the inelter was negligible. Looked at as an integrated process, the overall $\mathrm{NO}_{\mathrm{x}} \mathrm{y}$ ield is $(9.6 \%)(0.775)=7.4 \%$.

Because the majority of the $\mathrm{NO}_{\mathrm{x}}$ would be expected to be generated during the feed preparation process, it would be expected that USBM WHC2 and USBM WHC3 would have roughly the same $\mathrm{NO}_{x}$ yield as USBM WHCl. However, offgas data were not collected during the WHC2 and WHC3 runs to allow independent $\mathrm{NO}_{\mathrm{x}}$ yield estimates to be made for these runs.

\subsubsection{Vectra}

The Vectra slurry feed test (MT-2) used sucrose as a feed additive. The $2.6 \% N_{x}$ yield was the lowest yield obtained from any of the combined feed preparation/melter systems. The estimate was based on two offgas sampling periods during slurry feeding operation that were designated Run 1 and Run 2 . The feed characterization data were lacking and the results have been adjusted upward to account for a potential $\mathrm{NO}_{3}{ }^{-} / \mathrm{NO}_{2}^{-}$shortage in the feed corresponding to the apparent shortage of $\mathrm{Na}$.

Feed for the Vectra calcined feed test (MT-4) was prepared from LILW simulant with sucrose added which was calcined in a fluid bed calciner at $\sim 500{ }^{~ " C}$ with glass formers. The estimated fate of the $\mathrm{N}$ contained in the simulant $\mathrm{NO}_{3}^{-} / \mathrm{NO}_{2}^{-}$is provided in Table 3-5 for the calcination run. The conversion of $3.7 \%$ of the feed nitrogen in $\mathrm{NO}_{3}{ }^{-} / \mathrm{NO}_{2}^{-}$to $\mathrm{NO}_{x}$ is fairly

consistent with the data observed with slurry feeding of the meiter. However, the data appear to have been taken from a sample point downstream from a caustic scrubber which introduces uncertainty that $\mathrm{NO}_{2}$ may have been scrubbed before measurement. In any case, the reduction in the $\mathrm{NO}_{\mathrm{x}}$ emission during calcination was clearly significant. The data indicate more efficient conversion to $\mathrm{N}_{2}$ than was experienced in the USBM-1 test with much lower ammonia emissions.

\subsubsection{Duratek}

The Duratek test added urea to the feed and used a slurry feeding approach. The result of the 13\% NO yield was based on feed analysis and an

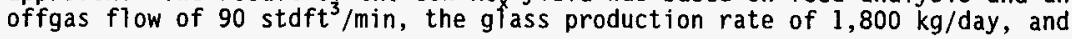


glass and feed analysis results. The result is reasonabiy consistent with results obtained by Duratek (Eaton 1995b). The $\mathrm{NO}_{\mathrm{x}}$ yield was calculated based only on the feed $\mathrm{NO}_{3}^{-} / \mathrm{NO}_{2}{ }^{-}$and does not include $\mathrm{N}$ contained in the urea.

\subsubsection{Envitco}

The Envitco test approach added activated carbon to the feed s1urry before a spray dry operation. The spray dry operation removed $20 \%$ to $30 \%$ (assumed $25 \%$ for calculations) of the $\mathrm{NO}_{3}^{-} / \mathrm{NO}_{2}^{-}$from the feed. However, information is unavailable on what fraction of the $\mathrm{NO}_{3}^{-} / \mathrm{NO}_{2}{ }^{-}$destroyed during feed preparation was evolved as $\mathrm{NO}_{\mathrm{x}}$. The melter itself evolved $33 \%$ of the $\mathrm{NO}_{3}^{-} / \mathrm{NO}_{2}^{-}$remaining in the spray-dried feed as $\mathrm{NO}_{x}$. When considering the feed preparation and melter as an integrated system, the melter accounts for a yield of $25 \%$ based on $\mathrm{NO}_{3}{ }^{-} / \mathrm{NO}_{2}{ }^{-}$in the feed to the spray dry process, which represents the minimum system $\mathrm{NO}_{\mathrm{x}}$ yield. An upper bound of $50 \% \mathrm{NO}_{\mathrm{x}}$ yield is estimated by assuming that all missing $\mathrm{NO}_{3}^{-} / \mathrm{NO}_{2}^{-}$in the spray-dried feed was evolved as $\mathrm{NO}_{\mathrm{x}}$.

\subsection{AMMONIA EMISSIONS}

Ammonia emissions were not monitored in all offgas runs. The data that are available are summarized below. Supporting calculations are provided in Appendix H.1.

\subsubsection{USBM}

As shown in Table 3-5, the reactions during feed preparation generated 9.8 mol of $\mathrm{NH}_{3}$ per $100 \mathrm{~mol}$ of $\mathrm{N}$ released as gas. The feed preparation process destroys $77.5 \%$ of the total feed $\mathrm{NO}_{3} / \mathrm{NO}_{2}^{-}$. Therefore, the $\mathrm{NH}_{3}$ yield is $(9.8)(0.775)=7.6 \%$ based on the total molar feed rate of $\mathrm{NO}_{3}{ }^{3} / \mathrm{NO}_{2}^{-}$fed to the process. The melter itself produces an $\mathrm{NH}_{3}$ yield of $0.9 \%$ based on the $\mathrm{NO}_{3}{ }^{-} / \mathrm{NO}_{2}{ }^{-}$remaining in the melter feed after the feed preparation process. The overalf $\mathrm{NH}_{3}$ yield based on the feed preparation and melter system is $7.6+(0.9)(0.225)=7.8 \%$.

\subsubsection{Vectra}

Vectra did not collect data on $\mathrm{NH}_{3}$ emissions for slurry feeding. However, data were available for $\mathrm{NH}_{3}$ emissions for the fluid bed calciner feed preparation run. As shown in Table $3-5,0.4 \%$ of the feed $N$ contained in $\mathrm{NO}_{3} / \mathrm{NO}_{2}$ was emitted as $\mathrm{NH}_{3}$ during calcination of the feed slurry.

\subsubsection{Duratek}

The Duratek $\mathrm{NH}_{3}$ yield is calculated neglecting the addition of urea to the feed and is based only on the $\mathrm{NO}_{3} / \mathrm{NO}_{2}{ }^{-}$content of the feed. On this basis, the melter emitted $\sim 8.8 \%$ of the molar feed $\mathrm{NO}_{3}{ }^{-} / \mathrm{NO}_{2}^{-}$as $\mathrm{NH}_{3}$. 
WHC-SD-WM-ER-517

Revision 0

\subsubsection{Envitco}

Data are unavailable from the spray dry process. However, looking only at the melter and using the $\mathrm{NO}_{3}{ }^{-} / \mathrm{NO}_{2}{ }^{-}$content of the dried feed, the $\mathrm{NH}_{3}$ yield is $4.1 \%$. 
WHC-SD-WM-ER-517

Revision 0

This page intentionally left blank. 


\subsection{REFERENCES}

Ecology, EPA, and DOE, 1994, Hanford Federal Facility Agreement and Consent Order, as amended, Washington State Department of Ecology,

U.S. Environmental Protection Agency, and U.S. Department of Energy, Olympia, Washington.

Eaton, W. C., 1995a, U.S. Bureau of Mines, Phase 1 Hanford Low-Level Waste Melter Tests: Final Report, WHC-SD-WM-VI-030, Rev. 0, Westinghouse Hanford Company, Richland, Washington.

Eaton, W. C., 1995b, GTS Duratek, Phase 1 Hanford Low-Level Waste Melter Tests: Final Report, WHC-SD-WM-VI-027, Rev. 0, Westinghouse Hanford Company, Richland, Washington.

Hendrickson, D. W., 1995, Evaluation of Plasma Melter Technology for Vitrification of High-Sodium Content Low-Level Radioactive Liquid Wastes--Demonstration Test Number 4 Final Test Report, WHC-SD-WM-VI-016, Rev. 0, Westinghouse Hanford Company, Richland, Washington.

Higley, B. A., 1995, Glass Melter System Technologies for Vitrification of High-Sodium Content Low-Level Radiaactive Liquid Wastes--Phase 1: SBS Demonstration with Simulated Low-Level Waste--Final Test Report, WHC-SD-WM-VI-024, Rev. 0, Westinghouse Hanford Company, Richland, Washington.

Mast, E. S., 1995, Low-Level Waste Vitrification Phase 1 Vendor Testing Sample Analyses Data, WHC-SD-WM-DP-131, Rev. O, Westinghouse Hanford Company, Richland, Washington.

Stegen, G. E., 1996, Vectra GSI, Inc. Low-Level Waste Melter Testing Phase 1 Test Report, WHC-SD-WM-VI-031, Rev. 0, Westinghouse Hanford Company, Richland, Washington.

Wilson, C. N., 1994, Evaluation of Melter System Technologies for Vitrification of High-Sodium Content Low-Level Radioactive Liquid Wastes, WHC-SD-WM-RD-044, Rev. 0, Westinghouse Hanford Company.

Wilson, C. N., 1996a, Low-Level Waste Vitrification Phase 1 Melter Vendor Demonstration Testing Summary Report, WHC-SD-WM-ER-498, Rev. 0 , Westinghouse Hanford Company, Richland, Washington.

Wilson, C. N., 1996b, Envitco, Inc., Low-Level Waste Phase 1 Melter Demonstration Test Report, WHC-SD-WM-VI-023, Rev. 0, Westinghouse Hanford Company, Richland, Washington. 
WHC-SD-WM-ER-517

Revision 0

This page intentionally left blank. 


\section{WHC-SD-WM-ER-517}

Revision 0

\subsection{GLOSSARY}

\section{ABBREYIATIONS AND ACRONYMS}

$\begin{array}{ll}\text { B\&W } & \text { Babcock \& Wilcox } \\ \text { Duratek } & \text { GTS Duratek, Inc. } \\ \text { Envitco } & \text { Envitco, Inc. } \\ \text { EPA } & \text { U.S. Environmental Protection Agency } \\ \text { LLW } & \text { low-level waste } \\ \text { USBM } & \text { U.S. Bureau of Mines } \\ \text { Vectra } & \text { Vectra Technologies, Inc. } \\ \text { WHC } & \text { Westinghouse Hanford Company } \\ \text { WSTC } & \text { Westinghouse Science and Technology Center }\end{array}$


WHC-SD-WM-ER-517

Revision 0

This page intentionally left blank.

GL-2 
WHC-SD-WM-ER-517

Revision 0

APPENDIX A

REVIEW OF BABCOCK AND WILCOX TEST REPORT 
WHC-SD-WM-ER-517

Revision 0

This page intentionally left blank. 


\section{DON'T SAY IT -- Write It!}

To: Greg Whyatt
Date: September 29, 1995

From: Charles Freeman

Subject: Review of Babcock \& Wilcox Off-Gas Data

(Revised 3/13/96 by Greg Whyatt)

The following review is of the Babcock \& Wilcox (B\&W) LLW Phase-1 testing off-gas results. The data used for this review came primarily from B\&W's final test report along with Westinghouse Hanford's analytical database. Although the B\&W report gave results from their scoping tests, only the 24-hour test data was reviewed for the present effort.

\section{Sample Selection:}

Table 1, attached, shows the samples collected during the 24 hour B\&W test along with the sampling time. Samples were originally collected to provide information for a total of five mass-balance points. However, the method-5 sample for the fourth mass-balance set was collected over one hour prior to it's corresponding glass sample. Additionally, the nearest feed sample was collected three hours later. As a result, the fourth mass-balance point was not calculated for this effort. The samples used for calculating the other four mass-balance points are identified in the sixth column of Table 1 . Unfortunately, a good chronology of events during the run was not given in the $B \& W$ report. A shutdown was discussed but the times at which it occurred were not clear. Therefore, Table 1 also shows some of the events which were described at run times as best as they could be determined.

\section{Condensible Off-gas Constituents:}

The non-condensible off-gas data was given but only as averaged points for a two hour time frame. The raw data for these values were also not included making it impossible to verify the calculations. As a result the non-condensible off-gas value calculations could not be verified beyond what was reported in Table B.2-4 of the B\&W report.

\section{Combined Entrainment \& Volatility (Carryover):}


There is considerable uncertainty in the carryover estimates made from the B\&W mass-balance run data, but it is expected that total carryover is on the order of $13 \%$ or greater. The analyses used to derive this estimate are described below.

The only feed, off-gas and glass stream mass data given in the B\&W report are totalized values reported in mass balance Figure 5-2, page 5-13. Unfortunately, two problems are apparent in B\&W's total mass balance analysis. First, B\&W shows the percent theoretical oxides in their feed slurry to be $56.47 \%$. There are no references as to how this value was obtained. By using the average loss on drying and loss on ignition values reported by Corning and USGS for the feed samples the percent theoretical oxides in the slurry becomes $46.3 \%$. This is a substantial difference from the value used by B\&W in their mass balance calculations. Second, $\mathrm{B} \& W$ reports a cumulative glass mass, however, test observations recall that the quenched glass frit was weighed wet. A similar fritting process used by Vortec produced cullet which held up as much as $23 \%$ water by mass after draining (PNL laboratory record book number 55332, pg. 41). It is assumed and concurred by Bruce Higley, that B\&W did not account for water hold-up when reporting their cumulative glass mass.

The uncertainty in the actual mass of glass produced (which results from the unknown amount of water hold-up) and the uncertainty in the slurry feed oxide loading prevent an accurate estimation of total entrainment and carryover. For the purpose of the analyses described herein, it is assumed that the $\mathrm{B} \& \mathrm{~W}$ glass exhibited a $23 \%$ water hold-up and the slurry contained $46.3 \mathrm{wt} \%$ glass-forming and waste simulant oxides. These calculations should be revisited if either of these estimates are subsequently improved.

Table 6 shows the steps taken to correct the reported $B \& W$ feed and glass totals, as described above. The revised feed and glass masses were used to estimate the total carryover during the 24-hour test. The total oxide carryover estimates are given in Tables 2 through 5. The total carryover values are reported as a percentage of the total expected glass production rate when $100 \mathrm{lbs} / \mathrm{h}$ of $46.3 \mathrm{wt} \%$ oxide feed is processed. The overall carryover estimates range from $9.8 \%$ to $15.9 \%$ of the total theoretical glass production. The average carryover rate is about $13.2 \%$. If the $56.47 \%$ oxide feed loading given by $\mathrm{B} \& \mathrm{~W}$ is used instead of $46.3 \%$, then these overall carryover estimates are about $82 \%$ as large as those shown in Tables 2 through 5 .

The data in Table 6 indicate that the overall feed oxide carryover was approximately $28 \%$. This is considerably higher than the $13.2 \%$ carryover estimate obtained from the Method 5 aerosol sampling data. The Table 6 carryover estimate, however, is highly-dependent on the assumed fraction of water hold-up in the product glass. If it is assumed that water hold-up was insignificant, then a calculation like that shown in Table 6 leads to an estimated carryover (combined entrainment and volatility) of only $6.5 \%$. A water hold-up fraction of about $7.2 \%$ 
yields a overall carryover estimate of $13.2 \%$ (which is consistent with the carryover estimate given by the Method 5 data). It is clear that an accurate measure of water hold-up is needed to refine the carryover estimate given in Table 6 .

Another estimate of total carryover was attempted by using B\&W's reported cumulative dry scrubber and baghouse masses (also reported in Figure 5-2 of the B\&W report). This value came out to be approximately $57 \%$ of the theoretical glass mass. However, since the caustic scrubber utilized a liquid slurry of calcium carbonate, I am suspicious of the equivalent oxide values shown entering and exiting that unit. Additionally, if the baghouse solids were: somewhat wet it could have resulted in this higher carryover estimate. Therefore, I would only recommend using the feed and glass masses and the Method 5 samples to estimate carryover.

\section{Fractional Volatilities:}

The feed analytical data provided by USGS was reported on a dry basis. Apparently, no data indicating water content was analyzed. Therefore, the subsequent calculations were made using only the Corning feed analytical data which did provide water content. Water content was critical for the total carryover calculations since the feed rate reported by B\&W was in pounds per hour of slurry. This same approach was used by the authors of the B\&W report. Unlike the feed data, the glass analyses from Corning were averaged with the USGS values for the calculations. The average of these two samples is denoted in the attached tables by an " $\mathrm{X}$ " in place of a " $U$ " or a " $C$ " in the sample number. Where only one analysis provided data for an analyte, the single value was used. In the case of fluoride, the feed target was selected as being more accurate that the analytical results. For feed iodine, the result from neutron activation analysis of USGS samples were used and ratioed back to the corning sample based on sodium content. The glass and feed values were used along with "tie" components to calculate constituent losses to the off-gas due to volatility.

Tables 2 through 5 show the results of the volatility and carryover calculations for mass balance sets 1,2,3, and 5, respectively. Each table shows volatility calculations for each constituent using five different tie components; $\mathrm{Al}, \mathrm{Fe}, \mathrm{Si}, \mathrm{Ca}$ and $\mathrm{Sr}$. These constituents were chosen since the volatility estimation equation assumes the tie component to have zero volatility. However, by looking through each mass balance set it is apparent that the values using the $\mathrm{Al}$ and $\mathrm{Fe}$ tie components are biased higher than each of the other components. It is believed that corrosion of the refractories if the cause of this bias. As a result, the values generated from the other tie components ( $\mathrm{Si}, \mathrm{Ca}$ and $\mathrm{Sr}$ ) were assumed to be more comparable for the present effort and, correspondingly, averaged for each mass balance set to produce the 
average volatilities shown (third to last column of Tables 2 through 5).

Two constituent showed consistently irregular volatility values. Barium and nickel were found to have highly negative estimated volatilities for all the mass balance/tie component sets. This would suggest more of this constituent in the glass than in feed. $\mathrm{NiO}$ is below detection limits in all feed samples and barium is at low levels in the feed. Although contamination sources have not been identified for these constituents, very little contamination could account for the result due to the small feed inventory. It is also possible that systematic analytical errors could contribute to this result.

Mass balance sets 1 and 3 showed highly negative volatility values for $\mathrm{Al}, \mathrm{Cr}, \mathrm{Fe}$, and $\mathrm{Ti}$, indicating much more of this material in the glass than in the feed. Indeed, the report describes the use of Plibrico 85-S and Shamrock 881-Plastic refractories. The Shamrock contains $9.8 \mathrm{wt} \% \mathrm{Cr}_{2} \mathrm{O}_{3}$ and both refractories contain aluminum as a major component with iron and titanium as minor components. One peculiarity is that the mass balance set in between these two sets (number 2) shows reasonable (around zero volatility) values for the $\mathrm{Al}$, $\mathrm{Cr}, \mathrm{Fe}$, and $\mathrm{Ti}$ constituents. One possible explanation is that the first mass balance set was taken while the refractory was still new(?) and sloughing material into the glass. Next, the second mass balance set was taken after a significant amount of material had been processed and a three hour feed stoppage (see Table 1) which cleaned the system of accumulated material. However, the third mass balance set was taken after cyclone gas inlet locations were modified to heat the front end more. This change could have produced more refractory corrosion on these "newly" heated areas and consequently generated the elevated levels of refractory constituents seen in the glass. After a number of hours the fifth mass balance set showed no apparent elevated levels of the refractory constinuents. This may have been a result of the large amount of feed material processed through the system since the third set.

Results provided for fluoride are based on USGS glass results. The USGS glass analysis consistently gave substantially higner fluoride concentrations while Corning analysis found the fluoride to be below detection. If the Corning results are correct then volatile loss of fluoride was higher than indicated.

All analytical results for $\mathrm{SO}_{3}$ in the glass were below detection limits. All calculations were performed using the detection limit. Therefore, the results from the tie component calculation provide the minimum loss while the maximum may range up to $100 \%$.

Feed analysis for $\mathrm{P}_{2} \mathrm{O}_{5}$ content consistently provided results that were substantially below the target composition. The results calculated in the attached sheet utilize the analyzed result for the feed and result in an average of a $70 \%$ increase in $\mathrm{P}_{2} \mathrm{O}_{5}$ content over the feed. If 
recalculated using the feed targets values, the volatile losses for the four mass balance periods are $26,41,-53$, and $26 \%$ respectively. Because the Plibrico Special $85-S$ and the Shamrock 881 Plastic refractories contain $\mathrm{P}_{2} \mathrm{O}_{5}\left(2.7 \mathrm{wt} \%\right.$ and 2.5 wt \% respectively) a source of $\mathrm{P}_{2} \mathrm{O}_{5}$ other than the feed is known to be present. Based on the results for $\mathrm{Al}$ and $\mathrm{Fe}$, the refractory corrosion appears to be lowest during mass balance periods 2 and 5 . Of these two periods the second mass balance period provides the highest volatility of $41 \%$. It is assumed that the loss result is highest during this period because the source from the refractory is minimum. As a result, it is suggested that an approximate estimate of $41 \%$ volatile loss for $\mathrm{P}_{2} \mathrm{O}_{5}$ be considered.

The average results for volatile components of interest are provided below:

\begin{tabular}{|l|l|l|}
\hline $\begin{array}{l}\text { Oxide } \\
\text { Component }\end{array}$ & $\begin{array}{l}\text { Average \% } \\
\text { volatility }\end{array}$ & Notes \\
\hline $\mathrm{B}_{2} \mathrm{O}_{3}$ & 67 & \\
\hline $\mathrm{Cs}_{2} \mathrm{O}$ & 83 & \\
\hline $\mathrm{K}_{2} \mathrm{O}$ & 51 & \\
\hline $\mathrm{MoO}_{3}$ & 60 & \\
\hline $\mathrm{Na}_{2} \mathrm{O}$ & 35 & \\
\hline $\mathrm{P}_{2} \mathrm{O}_{5}$ & $\sim 41 \%$ & $\begin{array}{l}\text { Maximum loss based on feed target values. Due to } \\
\text { refractory source, result is approximate. }\end{array}$ \\
\hline $\mathrm{SO}_{3}$ & $>51$ & All glass SO3 results are below detection limit \\
\hline $\mathrm{Cl}$ & 87 & \\
\hline F & 92 & $\begin{array}{l}\text { based on feed target and USGS glass data, higher losses } \\
\text { predicted using Corning data }\end{array}$ \\
\hline $\mathrm{I}$ & 94 & Neutron activation data \\
\hline
\end{tabular}

\section{Entrainment:}


The Method 5 sample elemental analyses were used, along with the feed sample data, to generate the fractional carryover values for each mass balance set. These values are shown in the last column of Tables 2 through 5 under the column title of "\% Carryover Based on Feed and Method 5 Samples." In order to estimated entrainment for the entire off-gas stream the carryover of a constituent with a low volatility, such as alumina, calcia, or silica, can typically be used. However, the silica carryover values for each mass balance set were low (ahout $1 \%$ to $3 \%$ ) compared to the alumina and calcia values (5\% to $11 \%$ ). This may be a result of a silica-based filter having been used for the Method 5 sampling. If this were the case the analytical error for silica in the Method 5 sample would be high. Mass balance set 5 showed low/reasonable volatility data for alumina and calcia. The corresponding carryover values were $9.4 \%$ and $7.4 \%$, respectively.

The average uniform entrainment is estimated to have been $8.7 \%$. This value was obtained by averaging the \% carryover estimates (right-most column) for alumina and calcia given in Tables 2 through 5. It must be stressed that the accuracy of this value depends to a sizeable extent on the actual amount of feed oxides processed by the melter during the mass balance test. 
WHC-SD-WM-ER-517

Revision 0

\section{Table 1. Samples Taken for $24 \mathrm{hr}$ B\&W Test}

\begin{tabular}{|c|c|c|c|c|c|c|}
\hline $\begin{array}{l}\text { Sample } \\
\text { Number }\end{array}$ & $\begin{array}{l}\text { Sampling } \\
\text { Medium }\end{array}$ & $\begin{array}{c}\text { 24hr Test } \\
\text { Date }\end{array}$ & \begin{tabular}{|c|} 
Sampling \\
Start \\
Time \\
\end{tabular} & $\begin{array}{c}\text { Sampling } \\
\text { End } \\
\text { Time } \\
\end{array}$ & $\begin{array}{c}\text { Mass } \\
\text { Balance } \\
\text { Calculation \# }\end{array}$ & Comments \\
\hline $\mathrm{B} 1 \mathrm{~F} 7-002 \mathrm{Cl}$ & Feed & $10 / 13 / 94$ & $13: 30$ & & 1 & Assumed Start \\
\hline B1F7-002U1 & Feed & $10 / 13 / 94$ & $13: 30$ & & 1 & Time of Run \\
\hline B1G9-002C1 & Glass & $10 / 13 / 94$ & $13: 30$ & & 1 & \\
\hline B1G9-002U1 & Glass & $10 / 13 / 94$ & $13: 30$ & & 1 & \\
\hline B1G9-005U2 & Glass & $10 / 13 / 94$ & $13: 30$ & & & \\
\hline B1G9-(1006U & Glass & $10 / 13 / 94$ & $13: 30$ & & & \\
\hline B1G9-008U3 & Glass & $10 / 13 / 94$ & $13: 30$ & & & \\
\hline B\&W-M15-R1 & Method 5 Filter & $10 / 13 / 94$ & 13:44 & $14: 14$ & 1 & \\
\hline $\mathrm{B} 1 \mathrm{~W} 10-002 \mathrm{Cl}$ & Baghouse & $10 / 13 / 94$ & 14:00 & & & \\
\hline B1D12-002Q1 & Dry Scrubber & $10 / 13 / 94$ & $14: 15$ & & 1 & Feed Stopped at \\
\hline B\&W-IMP-R 1 & Impactor (particle) & $10 / 13 / 94$ & $14: 43$ & $14: 49$ & 1 & $\sim 15: 40$ for 3 hours, \\
\hline BI W10-003C3 & Baghouse & $10 / 13 / 94$ & $19: 28$ & & & Accumulated Material \\
\hline $\mathrm{B} 1 \mathrm{G} 9-005 \mathrm{C} 2$ & Glass & $10 / 13 / 94$ & 19:50 & & 2 & Melted Away \\
\hline BIG9-005U2 & Glass & $10 / 13 / 94$ & $19: 50$ & & 2 & \\
\hline B\&W-IMP-R2 & Impactor (particle) & $10 / 13 / 94$ & $20: 09$ & $20: 14$ & 2 & \\
\hline B1F7-005C2 & Feed & $10 / 13 / 94$ & $20: 10$ & & 2 & \\
\hline B1F7-005U2 & Feed & $10 / 13 / 94$ & $20: 10$ & & 2 & \\
\hline $\mathrm{B} \& \mathrm{~W}-\mathrm{M} 5-\mathrm{R} 2$ & Method 5 Filter & $10 / 13 / 94$ & $20: 30$ & $21: 30$ & 2 & Off-gas Damper \\
\hline B1G9-006C & Glass & $10 / 13 / 94$ & $21: 30$ & & & Plugging Noted, \\
\hline B1G9-006G & Glass & $10 / 13 / 94$ & $21: 30$ & & & Gas Inlets Modified \\
\hline B1F7-01)8C3 & Feed & $10 / 14 / 94$ & $1: 20$ & & 3 & to Redistribute: Heat \\
\hline B1F7-008U3 & Feed & $10 / 14 / 94$ & $1: 20$ & & 3 & \\
\hline $\mathrm{B} 1 \mathrm{G} 9-008 \mathrm{C} 3$ & Glass & $10 / 14 / 94$ & $1: 25$ & & 3 & \\
\hline B 1 G9-008U3 & Glass & $10 / 14 / 94$ & $1: 25$ & & 3 & \\
\hline B1W10-004C3 & Baghouse & $10 / 14 / 94$ & $1: 40$ & & & \\
\hline B\&W-IMP-R3 & Impactor (particle) & $10 / 14 / 94$ & 1:44 & $1: 49$ & 3 & \\
\hline B\&W-M5-R3 & Method 5 Filter & $10 / 14 / 94$ & $2: 00$ & $3: 00$ & 3 & \\
\hline BHE-MR-R I & Baghouse & $10 / 14 / 94$ & 2:00 & $3: 00$ & 3 & \\
\hline $\mathrm{B} 1 \mathrm{D} 12-003 \mathrm{Q} 3$ & Dry Scrubber & $10 / 14 / 94$ & 2:04 & & 3 & \\
\hline B\&W-IMP-R4 & Impactor (particle) & $10 / 14 / 94$ & $6: 40$ & $6: 45$ & & \\
\hline B\&W-M5-R4 & Method 5 Filter & $10 / 14 / 94$ & $6: 55$ & $7: 55$ & & \\
\hline B1G9-011C4 & Glass & $10 / 14 / 94$ & 8:13 & & & \\
\hline BIG9-0I1G4 & Glass & $10 / 14 / 94$ & $8: 13$ & & & \\
\hline B\&W-IMP-R5 & Impactor (particle) & $10 / 14 / 94$ & $10: 18$ & $10: 23$ & 5 & \\
\hline B\&W-M 5-R5 & Method 5 Filter & $10 / 14 / 94$ & $10: 27$ & $11: 27$ & 5 & \\
\hline B1F7-013C & Feed & $10 / 14 / 94$ & $11: 30$ & & 5 & \\
\hline B $1 F 7-013 \mathrm{U}$ & Feed & $10 / 14 / 94$ & $11: 30$ & & 5 & \\
\hline BIMI4-00IC & Method 5 Probe & $10 / 14 / 94$ & $11: 30$ & & 5 & \\
\hline BID12-004Q4 & Dry Scrubber & $10 / 14 / 94$ & $11: 45$ & & 5 & \\
\hline BIW10-006C5 & Baghouse & $10 / 14 / 94$ & $11: 45$ & & & \\
\hline B1G9-013C5 & Glass & $10 / 14 / 94$ & $11: 55$ & & 5 & \\
\hline BIG9-0I3G5 & Glass & $10 / 14 / 94$ & $11: 55$ & & & \\
\hline BIG9-013U5 & Glass & $10 / 14 / 94$ & $11: 55$ & & 5 & \\
\hline B1G9-014C & Glass & $10 / 14 / 94$ & $14: 30$ & & & \\
\hline B1W11-001C & Baghouse & $10 / 18 / 94$ & $16: 10$ & & & \\
\hline
\end{tabular}


Table 2. Off-gas Calculations Based on Mass Balance Set \#1 (in weight \% unless otherwise specified)

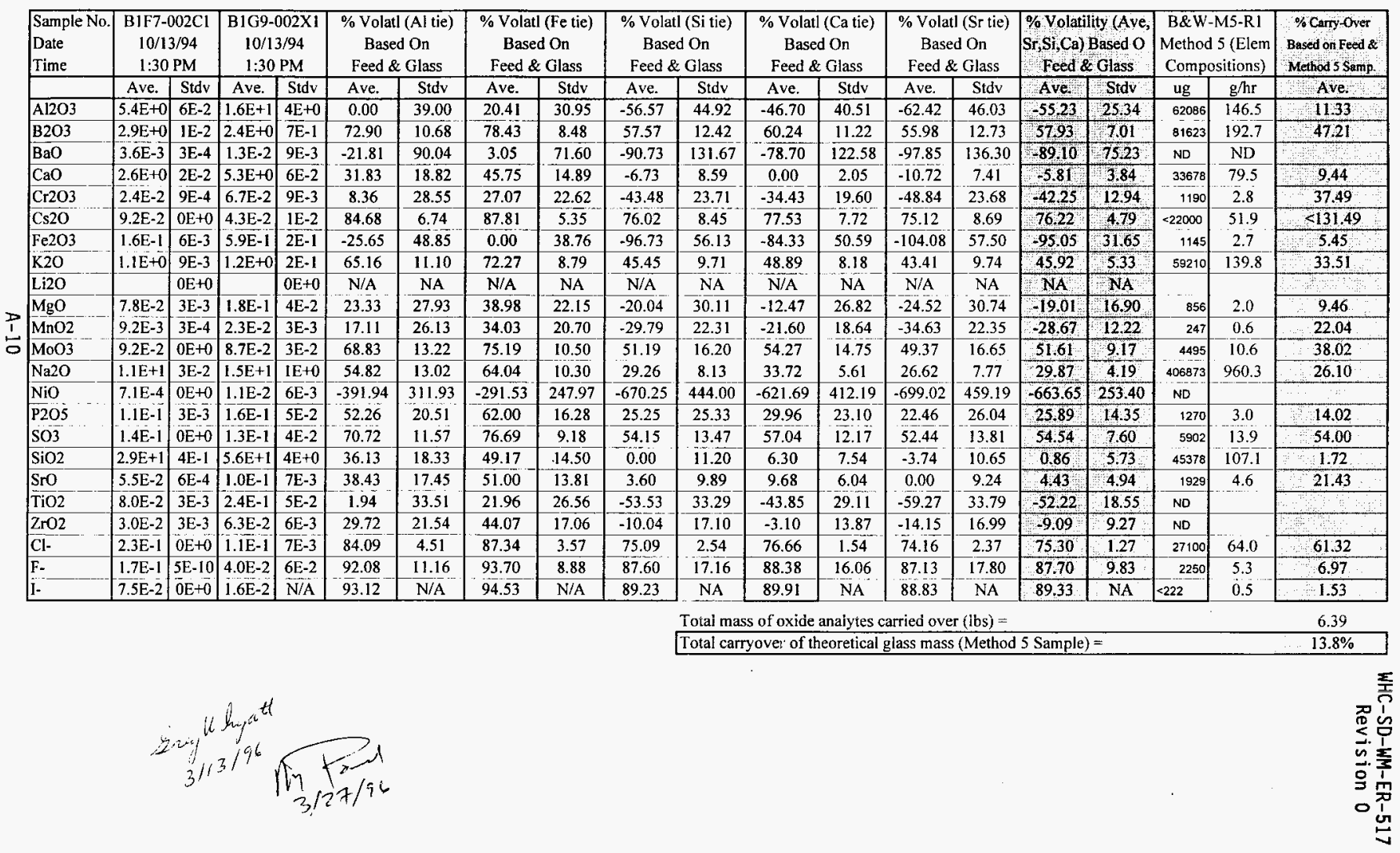


Table 3. Off-gas Calculations Based on Mass Balance Set \#2 (in weight $\%$ unless otherwise specified)

\begin{tabular}{|c|c|c|c|c|c|c|c|c|c|c|c|c|c|c|c|c|c|c|c|}
\hline \multirow[t]{2}{*}{\begin{tabular}{|l|} 
Sample No. \\
Date \\
Time \\
\end{tabular}} & \multicolumn{2}{|c|}{\begin{tabular}{|c|} 
BIF7-005C2 \\
$10 / 13 / 94$ \\
$8: 10 \mathrm{PM}$
\end{tabular}} & \multicolumn{2}{|c|}{$\begin{array}{c}\text { BIG9-005X2 } \\
10 / 13 / 94 \\
7: 50 \mathrm{PM}\end{array}$} & \multicolumn{2}{|c|}{$\begin{array}{l}\% \text { Volatl (Al tie) } \\
\text { Based On } \\
\text { Feed \& Glass }\end{array}$} & \multicolumn{2}{|c|}{$\begin{array}{c}\text { \% Volatl (Fe tie) } \\
\text { Based On } \\
\text { Feed \& Glass }\end{array}$} & \multicolumn{2}{|c|}{$\begin{array}{c}\text { \% Volatl (Si tie) } \\
\text { Based On } \\
\text { Feed \& Glass }\end{array}$} & \multicolumn{2}{|c|}{$\begin{array}{c}\% \text { Volatl (Ca tie) } \\
\text { Based On } \\
\text { Feed \& Glass }\end{array}$} & \multicolumn{2}{|c|}{$\begin{array}{l}\% \text { Volatl (Sr tie) } \\
\text { Based On } \\
\text { Feed \& Glass }\end{array}$} & \multicolumn{2}{|c|}{$\begin{array}{l}\text { \% Volatility (Ave, } \\
\text { Si, Ca, Sr) Based O } \\
\text { Feed \& Glass }\end{array}$} & \multicolumn{2}{|c|}{$\begin{array}{c}\text { B\&W-M5-R2 } \\
\text { Method 5 (Elem } \\
\text { Compositions) }\end{array}$} & \multirow{2}{*}{$\begin{array}{l}\text { \% Cary - Over } \\
\text { Based on Feed \& } \\
\text { Method } 5 \text { Samp. } \\
\text { Ave. }\end{array}$} \\
\hline & Ave. & Stdv & Ave. & Stdv & Ave. & Stdv & Ave. & Stdv & Ave. & $\overline{\text { Stdv }}$ & Ave. & $\overline{S t d v}$ & Ave. & Stdv & Ave: & Stdv & ug $E$ & $\mathrm{gE} / \mathrm{hr}$ & \\
\hline $\mathrm{Al} 2 \mathrm{O}^{3}$ & $5.6 \mathrm{E}+0$ & $1 \mathrm{E}-1$ & $1.2 \mathrm{E}+1$ & $7 \mathrm{E}-1$ & 0.00 & 9.52 & 24.56 & 17.93 & 2.53 & 6.88 & -6.39 & 8.17 & -23.92 & 9.99 & 9.26 & 488 & 51786 & 81.7 & 6.13 \\
\hline $\mathrm{B2} 23$ & $2.8 \mathrm{E}+0$ & $6 \mathrm{E}-3$ & $2.3 \mathrm{E}+0$ & & 61.94 & 2.56 & 71.29 & 6.54 & 62.91 & 0.80 & 59.51 & 1.50 & 52.84 & 2.09 & 58.42 & 090 & 101983 & 160.9 & 40.26 \\
\hline $\mathrm{BaO}$ & $3.6 \mathrm{E}-3$ & $3 \mathrm{E}-4$ & $9.5 \mathrm{E}-3$ & $3 \mathrm{E}-3$ & -27.37 & 43.54 & 3.91 & 38.94 & -24.15 & 41.69 & -35.51 & 45.69 & -57.85 & 53.36 & -39.17 & 2723 & ND & ND & \\
\hline $\mathrm{CaO}$ & $2 . \overline{\mathrm{E}+0}$ & $2 \mathrm{E}-2$ & $5.3 \mathrm{E}+0$ & $2 E-1$ & 6.00 & 7.22 & 29.09 & 16.37 & 8.38 & 3.92 & 0.00 & 5.24 & -16.48 & 6.73 & 2,70 & 3.13 & 25958 & 40.9 & 4.70 \\
\hline $\mathrm{Cr} 2 \mathrm{O}$ & $2.3 \mathrm{E}-2$ & $4 \mathrm{E}-4$ & $4.2 \mathrm{E}-2$ & $\overline{\mathrm{IE}-2}$ & 14.68 & 20.67 & 35.63 & 20.97 & 16.84 & 19.44 & 9.23 & 21.39 & -5.73 & 25.05 & 678 & 12.75 & 1187 & 1.9 & 25.71 \\
\hline $\mathrm{Cs} 2 \mathrm{O}$ & $8.5 \mathrm{E}-2$ & $0 \mathrm{E}+0$ & $3.5 \mathrm{E}-2$ & $8 \mathrm{E}-3$ & 80.45 & 4.79 & 85.25 & 4.83 & 80.94 & 4.50 & 79.20 & 4.96 & 75.77 & 5.80 & 78,64 & 295 & $<22000$ & 34.7 & $<95.07$ \\
\hline $\mathrm{Fe} \overline{20} 3$ & $1.6 \mathrm{E}-1$ & $3 \mathrm{E}-3$ & $4.4 \mathrm{E}-1$ & $1 \mathrm{E}-1$ & -32.55 & 31.50 & 0.00 & 32.23 & -29.20 & 29.57 & -41.02 & 32.56 & -64.27 & 38.14 & 44.83 & 1941 & 521 & 0.8 & 1.66 \\
\hline K2O & $1.0 \mathrm{E}+0$ & $7 \mathrm{E}-3$ & $1.1 \mathrm{E}+0$ & $8 \mathrm{E}-2$ & 48.91 & 5.11 & 61.46 & 9.23 & 50.21 & 3.83 & 45.65 & 4.49 & 36.69 & 5.46 & 4418 & 2,68 & 76100 & 120.0 & 30.92 \\
\hline $\mathrm{Li} 2 \mathrm{O}$ & & $0 \mathrm{E}+0$ & & $0 \mathrm{E}+0$ & N/A & N/A & N/A & N/A & $\mathrm{N} / \mathrm{A}$ & N/A & N/A & N/A & $\mathrm{NA}$ & NA & $\mathrm{NA}$ & $\mathrm{N} / \mathrm{A}$ & & & \\
\hline $\mathrm{MgO}$ & $7.8 \mathrm{E}-2$ & $0 \mathrm{E}+0$ & $1.7 \mathrm{E}-1$ & $9 \mathrm{E}-3$ & -0.56 & 8.83 & 24.14 & 17.81 & 1.99 & 5.91 & -6.98 & 7.22 & -24.62 & 8.94 & 9.87 & 4.31 & 686 & 1.1 & 5,06 \\
\hline $\mathrm{MnO} 2$ & $9.2 \mathrm{E}-3$ & $1 \mathrm{E}-3$ & $2.1 \mathrm{E}-2$ & $6 \bar{E}-3$ & -8.29 & 33.82 & 18.30 & 31.10 & -5.55 & 32.27 & -15.21 & 35.40 & \begin{tabular}{|l}
-34.20 \\
\end{tabular} & 41.36 & -18.32 & 21.09 & 157 & 0.2 & 9.35 \\
\hline $\mathrm{MoO} 3$ & $9.1 \mathrm{E}-2$ & $3 \mathrm{E}-3$ & $8.2 \mathrm{E}-2$ & $6 \mathrm{E}-3$ & 57.05 & 4.60 & 67.60 & 7.86 & 58.14 & 3.60 & 54.31 & 4.16 & 46.78 & 5.02 & 53.08 & 248 & 5110 & 8.1 & 29.30 \\
\hline $\mathrm{Na} 2 \mathrm{O}$ & $1.1 \mathrm{E}+1$ & $3 \mathrm{E}-2$ & $1.3 \mathrm{E}+1$ & $7 \mathrm{E}-1$ & 40.11 & 5.13 & 54.82 & 10.57 & 41.63 & 3.33 & 36.29 & 4.11 & 25.78 & 5.12 & 34.57 & 2.45 & 434873 & 686.0 & 19.11 \\
\hline $\mathrm{NiO}$ & $7.1 \mathrm{E}-4$ & $0 \mathrm{E}+0$ & $3.5 \mathrm{E}-3$ & 4E-3 & -134.64 & 251.65 & -77.01 & 193.72 & -128.70 & 244.84 & -149.62 & 267.35 & -190.77 & 311.50 & -156.36 & 159.32 & NO & & 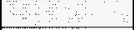 \\
\hline P2O5 & $1.1 \mathrm{E}-1$ & $4 \mathrm{E}-3$ & $1.2 \mathrm{E}-1$ & $4 \mathrm{E}-2$ & 45.68 & 17.29 & 59.02 & 15.80 & 47.06 & 16.51 & 42.21 & 18.11 & 32.69 & 21.15 & 40.65 & 10.79 & 1550 & 2.4 & 11.73 \\
\hline $\mathrm{SO} 3$ & $1.2 \mathrm{E}-1$ & $0 E+0$ & $1.3 \mathrm{E}-1$ & $4 \mathrm{E}-2$ & 52.33 & 13.86 & 64.04 & 13.06 & 53.54 & 13.18 & 49.29 & 14.46 & 40.93 & 16.91 & 47.92 & 8.62 & 8267 & 13.0 & 57.43 \\
\hline $\mathrm{SiO2}$ & $2.9 \mathrm{E}+\mathrm{l}$ & $5 \mathrm{E}-1$ & $6.3 \mathrm{E}+1$ & $7 \mathrm{E}-1$ & -2.60 & 7.24 & 22.60 & 17.72 & 0.00 & 3.02 & -9.15 & 4.66 & -27.14 & 6.26 & 12.10 & 2.79 & 34349 & 54.2 & 0.87 \\
\hline Sro & $5.8 \mathrm{E}-2$ & $1 \mathrm{E}-3$ & $9.8 \mathrm{E}-2$ & $4 \mathrm{E}-3$ & 19.31 & 6.50 & 39.12 & 14.13 & 21.35 & 3.87 & 14.15 & 4.96 & 0.00 & 6.27 & 11.83 & 2.96 & 1673 & 2.6 & 11.95 \\
\hline TiO2 & 8.7E-2 & $4 \mathrm{E}-3$ & $2.0 \mathrm{E}-1$ & $2 \mathrm{E}-2$ & -7.10 & 15.80 & 19.20 & 21.25 & -4.39 & 13.88 & -13.94 & 15.54 & -32.72 & 18.39 & 17.02 & 9.26 & ND & & 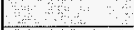 \\
\hline $\mathrm{ZrO} 2$ & $3.0 \mathrm{E}-2$ & IE-3 & \begin{tabular}{|l|}
$7.0 \mathrm{E}-2$ \\
\end{tabular} & $5 \mathrm{E}-3$ & -11.73 & 12.56 & 15.71 & 20.65 & -8.90 & 10.07 & -18.87 & 11.57 & \begin{tabular}{|l|}
-38.46 \\
\end{tabular} & 13.89 & 22.08 & 6.90 & ND & & \\
\hline $\mathrm{Cl}-$ & $2.3 \mathrm{E}-1$ & $0 \mathrm{E}+0$ & $7.0 \mathrm{E}-2$ & $3 \mathrm{E}-3$ & 85.49 & 1.14 & 89.06 & 2.53 & 85.86 & 0.65 & 84.57 & 0.85 & 82.02 & 1.08 & 84.15 & 0.51 & 39901 & 62.9 & 60.33 \\
\hline F- & $1.6 \mathrm{E}-1$ & $O E+0$ & $2.0 \mathrm{E}-2$ & $3 \mathrm{E}-2$ & 94.18 & 7.86 & 95.61 & 6.01 & 94.33 & 7.65 & 93.81 & 8.35 & 92.79 & 9.73 & 93.64 & 4.98 & 2900 & 4.6 & 6.15 \\
\hline I- & $8.4 \mathrm{E}-2$ & $0 \mathrm{E}+0$ & $1.1 \mathrm{E}-2$ & $\mathrm{~N} / \mathrm{A}$ & 94.01 & N/A & 95.48 & $\mathrm{~N} / \mathrm{A}$ & 94.17 & $\bar{N} / \mathrm{A}$ & 93.63 & $\mathrm{~N} / \mathrm{A}$ & 92.58 & $\mathrm{NA}$ & 93.46 & $\mathrm{NA}$ & $<222$ & 0.4 & 0.92 \\
\hline & & & & & & & & & & Total ma & fox & alyt & Tied o & $(\mathrm{bs})=$ & & & & & 4.58 \\
\hline & & & & & & & & & & Total car & over of & eoretical & lass mas: & (Method & d 5 Samp & & & & $9.9 \%$ \\
\hline
\end{tabular}


Table 4. Off-gas Calculations Based on Mass Balance Set $\# 3$ (in weight $\%$ unless otherwise specified)

\begin{tabular}{|c|c|c|c|c|c|c|c|c|c|c|c|c|c|c|c|c|c|c|c|}
\hline \multirow[t]{2}{*}{\begin{tabular}{|l} 
Sample No. \\
Date \\
Time \\
\end{tabular}} & \multicolumn{2}{|c|}{$\begin{array}{c}\mathrm{B} 1 \mathrm{~F} 7-008 \mathrm{C} 3 \\
10 / 14 / 94 \\
1: 20 \mathrm{AM} \\
\end{array}$} & \multicolumn{2}{|c|}{$\begin{array}{c}\mathrm{B} 1 \mathrm{G} 9-008 \mathrm{X} 3 \\
10 / 14 / 94 \\
1: 25 \mathrm{AM} \\
\end{array}$} & \multicolumn{2}{|c|}{$\begin{array}{c}\text { \% Volatl (Al tie) } \\
\text { Based On } \\
\text { Feed \& Glass }\end{array}$} & \multicolumn{2}{|c|}{$\begin{array}{c}\% \text { Volatl (Fe tie) } \\
\text { Based On } \\
\text { Feed \& Glass }\end{array}$} & \multicolumn{2}{|c|}{$\begin{array}{c}\% \text { Volatl (Si tie) } \\
\text { Based On } \\
\text { Feed \& Glass } \\
\end{array}$} & \multicolumn{2}{|c|}{$\begin{array}{c}\% \text { Volatl (Ca tie) } \\
\text { Based On } \\
\text { Feed \& Glass }\end{array}$} & \multicolumn{2}{|c|}{\begin{tabular}{|c|}
$\%$ Volatl (Sr tie) \\
Based On \\
Feed \& Glass \\
\end{tabular}} & \multicolumn{2}{|c|}{$\begin{array}{l}\text { \% Volatility (Ave, } \\
\text { Si,Ca,Sr) Based O } \\
\text { Feed \& Glass }\end{array}$} & \multicolumn{2}{|c|}{$\begin{array}{l}\text { B\&W-M5-R3 } \\
\text { Method } 5 \text { (Elem } \\
\text { Compositions) }\end{array}$} & \multirow{2}{*}{$\begin{array}{l}\text { \% Cary-Over } \\
\text { Based on feed \& } \\
\text { Method } 5 \text { Samp } \\
\text { Ave. }\end{array}$} \\
\hline & Ave. & Stdv & Ave. & Stdv & Ave. & Stdv & Ave. & Stdv & Ave. & Stdv & Ave. & Stdv & Ave. & Stdv & Ave. & Stdv & ppm E & $\mathrm{g} \mathrm{E} / \mathrm{hr}$ & \\
\hline $\mathrm{A} / 2 \mathrm{O} 3$ & $5.6 \mathrm{E}+0$ & \begin{tabular}{|l|} 
IE-1 \\
\end{tabular} & $1.9 \mathrm{E}+\mathrm{I}$ & $6 \mathrm{E}+0$ & 0.00 & 43.77 & 41.29 & 18.57 & -87.41 & 69.66 & -48.51 & 50.41 & -90.69 & 72.54 & -7555 & 37.50 & 93500 & 146.6 & 10.93 \\
\hline $\mathrm{B} 2 \mathrm{O} 3$ & $2.9 \mathrm{E}+0$ & $2 \mathrm{E}-2$ & $7.5 \mathrm{E}-1$ & & 92.49 & 2.33 & 95.59 & 0.29 & 85.92 & $2 . \overline{90}$ & 88.85 & 1.56 & 85.68 & 3.17 & 86.82 & 1.52 & 132983 & 208.5 & 5116 \\
\hline $\mathrm{BaO}$ & $3.6 \mathrm{E}-3$ & $3 \mathrm{E} \sim 4$ & $.1 \mathrm{E}-2$ & $3 \mathrm{E}-3$ & 14.46 & 35.05 & 49.78 & 13.88 & -60.30 & 54.25 & -27.02 & 38.44 & -63.11 & 56.75 & -50.14 & 29.14 & ND & ND & 8 \\
\hline $\mathrm{CaO}$ & $2.7 \mathrm{E}+0$ & $6 \mathrm{E}-2$ & $6.2 \overline{\mathrm{E}+0}$ & $8 \mathrm{E}-1$ & 32.66 & 22.86 & 60.47 & 6.09 & -26.20 & 31.38 & 0.00 & 19.72 & -28.41 & 33.57 & 18.20 & 16.67 & 56858 & 89.1 & 10.32 \\
\hline $\mathrm{Cr} 2 \mathrm{O}^{3}$ & $2.4 \mathrm{E}-2$ & $2 \mathrm{E}-3$ & $1.7 \mathrm{E}-1$ & $1 \mathrm{E}-1$ & -110.44 & 155.97 & -23.55 & 83.59 & -294.40 & 277.73 & -212.52 & 214.92 & -301.29 & 284.44 & 269,40 & 150.64 & 1507 & 2.4 & 22 \\
\hline $\mathrm{Cs} 2 \mathrm{O}$ & $8.6 \mathrm{E}-2$ & $0 \mathrm{E}+0$ & $1.5 \mathrm{E}-2$ & 3E-3 & 95.08 & 1.75 & 97.11 & 0.54 & 90.78 & 2.49 & 92.69 & 1.64 & 90.61 & 2.65 & 9136 & 1.33 & $<22000$ & 34.5 & $<3.82$ \\
\hline $\mathrm{Fe} 2 \mathrm{O} 3$ & $1.6 \mathrm{E}-1$ & $3 \mathrm{E}-3$ & $9.2 \mathrm{E}-1$ & $6 \mathrm{E}-2$ & -70.33 & 53.88 & 0.00 & 9.24 & -219.22 & 68.95 & -152.95 & 38.95 & -224.80 & 74.90 & -198.99 & 36.33 & 776 & 1.2 & 2.43 \\
\hline $\mathrm{K} 2 \mathrm{O}$ & $1.0 \mathrm{E}+0$ & $9 \mathrm{E}-4$ & $7.8 \mathrm{E}-1$ & $9 \bar{E}-2$ & 78.09 & 7.25 & 87.14 & $1 . \overline{73}$ & 58.94 & 9.73 & 67.46 & 5.93 & 58.22 & 10.46 & 61.54 & 5.16 & 93200 & $14 \overline{6}$. & 37.37 \\
\hline $\mathrm{Li} 2 \mathrm{O}$ & & $0 \mathrm{E}+0$ & & $0 \mathrm{E}+0$ & $\mathrm{~N} / \bar{A}$ & $\mathrm{~N} / \mathrm{A}$ & N/A & N/A & $\mathrm{N} / \bar{A}$ & $\mathrm{~N} / \mathrm{A}$ & N/A & N/A & NA & NA & N/A & N/A & & & \\
\hline $\mathrm{MgO}$ & $7.9 \mathrm{E}-2$ & $0 \mathrm{E}+0$ & $2.9 \mathrm{E}-1$ & $7 \mathrm{E}-2$ & -5.53 & 41.79 & 38.04 & 15.83 & -97.78 & 63.60 & -56.72 & 44.46 & -101.24 & 66.72 & 85.24 & 34.11 & 1250 & $2 . \overline{0}$ & 9.10 \\
\hline $\mathrm{MnO} 2$ & $1.1 \mathrm{E}-2$ & $3 \mathrm{E}-4$ & $2.5 \mathrm{E}-2$ & $1 \mathrm{E}-3$ & 33.47 & 20.99 & 60.94 & 3.50 & -24.68 & 26.78 & 1.20 & 15.05 & -26.86 & 29.11 & -16.78 & 14.11 & 214 & 0.3 & 0.90 \\
\hline $\mathrm{MoO} 3$ & $8.4 \mathrm{E}-2$ & $3 \mathrm{E}-3$ & $3.2 \mathrm{E}-2$ & $1 \mathrm{E}-2$ & 88.95 & 5.01 & $9 \overline{3} . \overline{52}$ & 2.19 & 79.30 & 8.08 & $8 \overline{3} . \overline{60}$ & 5.90 & 78.94 & 8.40 & 80.61 & 4.36 & 6280 & 9.8 & 38.54 \\
\hline $\mathrm{Na} 2 \mathrm{O}$ & $1.1 \mathrm{E}+1$ & $3 \mathrm{E}-2$ & $1.2 \mathrm{E}+1$ & $3 \mathrm{E}+0$ & 66.10 & 12.59 & 80.10 & 4.29 & 36.47 & 18.47 & 49.66 & 12.49 & 35.36 & 19.50 & 40.50 & 9.88 & 675873 & 1059.6 & 29.51 \\
\hline $\mathrm{NiO}$ & $7.2 \mathrm{E}-4$ & $0 \mathrm{E}+0$ & $1.3 \mathrm{E}-2$ & $7 \mathrm{E}-3$ & -409.14 & 323.56 & -198.91 & 167.05 & -854.18 & 564.86 & -656.10 & 432.70 & -870.87 & 580.06 & 793.72 & 306.01 & ND & & \\
\hline P2O5 & $1.1 \mathrm{E}-1$ & $2 \mathrm{E}-3$ & 3.2E-1 & $1 \mathrm{E}-1$ & 12.76 & 38.77 & 48.78 & 16.67 & -63.50 & 62.05 & $-29.5 \overline{6}$ & 45.08 & -66.36 & 64.55 & -53.14 & 33.42 & 2240 & 3.5 & 16.85 \\
\hline $\mathrm{SO3}$ & $1.3 \mathrm{E}-1$ & $0 \mathrm{E}+0$ & $1.3 \mathrm{E}-1$ & $4 \mathrm{E}-2$ & 70.84 & 12.23 & 82.88 & 4.97 & 45.35 & 19.12 & 56.70 & 13.65 & 44.39 & 19.96 & 48.81 & 10.28 & 2993 & 4.7 & 0.66 \\
\hline $\mathrm{SiO} 2$ & $3.1 \mathrm{E}+1$ & $3 E-1$ & $5.6 \mathrm{E}+1$ & $1 \mathrm{E}+1$ & 46.64 & 19.83 & 68.67 & 6.77 & 0.00 & 29.11 & 20.76 & 19.70 & -1.75 & 30.74 & 6.34 & 15.57 & 133791 & 209.7 & 3.22 \\
\hline SrO & $5.7 \mathrm{E}-2$ & $1 \mathrm{E}-3$ & $1.0 \mathrm{E}-1$ & $2 \mathrm{E}-2$ & 47.56 & 19.95 & 69.21 & 7.10 & 1.72 & 29.69 & 22.12 & 20.36 & 0.00 & 31.27 & 7.95 & 15.90 & 2993 & 4.7 & 21.36 \\
\hline TiO2 & 9.9E-2 & 9E-4 & $3.3 \mathrm{E}-1$ & $2 \mathrm{E}-2$ & 4.08 & 30.36 & 43.68 & 5.23 & -79.77 & 38.86 & -42.45 & 21.97 & -82.92 & 42.21 & -68.38 & 20.48 & ND & & \\
\hline $\mathrm{ZrO} 2$ & $3.6 \mathrm{E}-1$ & $2 \mathrm{E}-3$ & $6.7 \mathrm{E}-2$ & $2 \mathrm{E}-2$ & 94.58 & 2.43 & 96.82 & 1.06 & 89.85 & 3.91 & 91.96 & 2.85 & 89.67 & 4.07 & 90.49 & 2.11 & ND & & \\
\hline $\mathrm{Cl}-$ & $2 . \overline{\mathrm{E}}-1$ & $\overline{0 \mathrm{E}+0}$ & $1.5 \mathrm{E}-2$ & IE-2 & 98.38 & 1.18 & 99.05 & 0.63 & 96.96 & 2.10 & 97.59 & 1.62 & 96.91 & 2.15 & 97.16 & 1.14 & 41908 & 65.7 & 53.64 \\
\hline F- & $1.6 \mathrm{E}-1$ & $0 \mathrm{E}+0$ & $2.0 \mathrm{E}-2$ & $3 \mathrm{E}-2$ & 96.44 & 4.93 & 97.91 & 2.82 & 93.33 & 9.10 & $94 . \overline{7 l}$ & 7.17 & 93.21 & 9.28 & 93.75 & 4.95 & 53000 & 83.1 & 111.77 \\
\hline I- & $7.3 \mathrm{E}-2$ & $0 \mathrm{E}+0$ & $2.5 \mathrm{E}-3$ & $0 \mathrm{E}+0$ & 99.00 & N/A & 99.41 & N/A & 98.12 & $\mathrm{~N} / \mathrm{A}$ & 98.51 & $\mathrm{~N} / \mathrm{A}$ & 98.09 & NA & 98.24 & NA & $<222$ & 0.3 & 1.05 \\
\hline & & & & & & & & & & otal & $f \circ$ & & ried & $a s)=$ & & & & & 7.41 \\
\hline
\end{tabular}

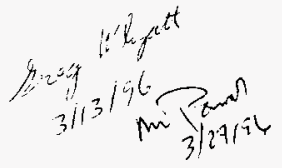


Table 5. Off-gas Calculations Based on Mass Balance Set \#5 (in weight $\%$ unless otherwise specified)

\begin{tabular}{|c|c|c|c|c|c|c|c|c|c|c|c|c|c|c|c|c|c|c|c|}
\hline \multirow[t]{2}{*}{\begin{tabular}{|l} 
Sample No. \\
Date \\
Time \\
\end{tabular}} & \multicolumn{2}{|c|}{$\begin{array}{c}\text { B1F7-013C } \\
10 / 14 / 94 \\
11: 30 \mathrm{AM} \\
\end{array}$} & \multicolumn{2}{|c|}{$\begin{array}{c}\text { B1G9-013XS } \\
10 / 14 / 94 \\
11: 55 \mathrm{AM}\end{array}$} & \multicolumn{2}{|c|}{$\begin{array}{c}\text { \%olatl (Al tie) } \\
\text { Based On } \\
\text { Feed \& Glass } \\
\end{array}$} & \multicolumn{2}{|c|}{$\begin{array}{c}\% \text { Volatl (Fe tie) } \\
\text { Based On } \\
\text { Feed \& Glass }\end{array}$} & \multicolumn{2}{|c|}{$\begin{array}{c}\% \text { Volatl (Si tie) } \\
\text { Based On } \\
\text { Feed \& Glass }\end{array}$} & \multicolumn{2}{|c|}{$\begin{array}{c}\% \text { Volatl (Ca tie) } \\
\text { Based On } \\
\text { Feed \& Glass }\end{array}$} & \multicolumn{2}{|c|}{$\begin{array}{c}\% \text { Volatl ( } \mathrm{Sr} \text { tie) } \\
\text { Based On } \\
\text { Feed \& Glass }\end{array}$} & \multicolumn{2}{|c|}{$\begin{array}{l}\% \text { Volatility (Ave, } \\
\text { Al,Si,Ca) Based O } \\
\text { Feed \& Glass }\end{array}$} & \multicolumn{2}{|c|}{\begin{tabular}{|c|} 
B\&W-M5-R5 \\
Method 5 (Elem \\
Compositions) \\
\end{tabular}} & \multirow[t]{2}{*}{\begin{tabular}{|l|}
$\%$ Carty Over \\
Based on Feed \& \\
Method 5 samp \\
Ave.
\end{tabular}} \\
\hline & Ave. & Stdv & Ave. & Stdv & Ave. & Stdv & Ave. & Stdv & Ave. & Stdv & Ave. & Stdv & Ave. & Stdv & Ave & Stdv & ppm E & $\mathrm{g} \mathrm{E} / \mathrm{hr}$ & \\
\hline$\overline{\mathrm{Al} 2 \mathrm{O3}}$ & $5.7 \mathrm{E}+0$ & $5 \mathrm{E}-2$ & $1.3 \mathrm{E}+1$ & $1 E+0$ & 0.00 & 11.92 & 0.93 & 16.54 & -8.58 & 10.64 & -5.50 & 9.09 & -25.65 & 15.50 & 4.69 & 6.96 & 78286 & 127.3 & 9.37 \\
\hline $\mathrm{B} 2 \mathrm{O} 3$ & $2.7 \mathrm{E}+0$ & $\overline{1 E-2}$ & $2.1 E+0$ & & 65.19 & 2.94 & 65.51 & 4.97 & 62.20 & 1.90 & 63.27 & 0.69 & $56 . \overline{26}$ & 3.95 & 63.55 & 1,48 & 119983 & 195.1 & 50.45 \\
\hline $\mathrm{BaO}$ & $3.6 \mathrm{E}-3$ & \begin{tabular}{|l|}
$4 \mathrm{E}-4$ \\
\end{tabular} & $9.5 \mathrm{E}-3$ & $1 \mathrm{E}-3$ & -20.38 & 22.97 & -19.27 & 26.68 & -30.71 & 23.31 & -27.00 & 21.86 & -51.26 & 29.26 & -26.03 & 14.44 & ND & ND & , \\
\hline $\mathrm{CaO}$ & $2.7 \mathrm{E}+0$ & 2E-2 & $5.7 \mathrm{E}+0$ & $9 \mathrm{E}-2$ & 5.21 & 8.17 & 6.09 & 13.64 & -2.92 & 5.48 & 0.00 & 2.55 & -19.10 & 10.95 & 0.76 & 4,17 & 39758 & 64.7 & 7.38 \\
\hline $\mathrm{Cr} 2 \mathrm{O} 3$ & $2.3 \mathrm{E}-2$ & 7E-4 & $4.9 \mathrm{E}-2$ & $1 \mathrm{E}-2$ & 2.98 & 29.94 & 3.88 & 31.72 & -5.34 & 31.72 & -2.35 & 30.45 & -21.91 & 37.83 & 1.57 & 19.33 & 1457.1 & 2.4 & 33.37 \\
\hline$\overline{C s 2 O}$ & $8.6 \mathrm{E}-2$ & $0 \mathrm{E}+0$ & $2.8 \mathrm{E}-2$ & $2 \mathrm{E}-3$ & 85.48 & 1.58 & 85.61 & 2.29 & 84.23 & 1.34 & 84.68 & 1.08 & 81.76 & 2.06 & 84.80 & 0.90 & $<22000$ & 35.8 & $<97.47$ \\
\hline $\mathrm{Fe} 2 \mathrm{O3}$ & $3.1 \mathrm{E}-1$ & \begin{tabular}{|l|}
$9 \mathrm{E}-3$ \\
\end{tabular} & $6.9 \mathrm{E}-1$ & $1 \mathrm{E}-1$ & -0.93 & 16.85 & 0.00 & 20.37 & -9.59 & 16.71 & -6.48 & 15.46 & -26.82 & 21.55 & -5.67 & 10.45 & 7675.1 & 12.5 & 12.80 \\
\hline $\mathrm{K} 2 \mathrm{O}$ & $1.0 \mathrm{E}+0$ & $3 \mathrm{E}-3$ & $1.0 \mathrm{E}+0$ & $5 \mathrm{E}-2$ & 54.90 & 4.38 & 55.32 & 6.79 & 51.03 & 3.41 & 52.42 & 2.46 & 43.33 & 5.80 & 52.78 & 2.39 & 90100 & 146.5 & 37.79 \\
\hline $\mathrm{Li} 2 \mathrm{O}$ & & $0 \mathrm{E}+0$ & & $0 \mathrm{E}+0$ & $\mathrm{~N} / \mathrm{A}$ & $\mathrm{N} / \mathrm{A}$ & $\mathrm{N} / \mathrm{A}$ & $\mathrm{N} / \mathrm{A}$ & NiA & N/A & N/A & $\mathrm{N} / \mathrm{A}$ & NA & $\mathrm{NA}$ & N/A & $\mathrm{N} / \mathrm{A}$ & & & \\
\hline $\mathrm{MgO}$ & $7.9 \mathrm{E}-2$ & $0 \mathrm{E}+0$ & 1.7E-1 & $2 E-2$ & 2.08 & 12.97 & 2.99 & 17.14 & -6.32 & 12.10 & -3.30 & 10.72 & -23.04 & 16.77 & -2.51 & 7.76 & 1060 & 1.7 & 8.01 \\
\hline $\mathrm{MnO} 2$ & $9.3 \mathrm{E}-3$ & 1E-3 & $2.1 \mathrm{E}-2$ & $6 \mathrm{E}-3$ & -2.35 & 34.46 & -1.40 & 36.14 & -11.13 & 36.65 & $-7 . \overline{98}$ & 35.25 & -28.60 & 43.49 & 7.15 & 22.30 & 208 & 0.3 & 12.70 \\
\hline $\mathrm{MoO} 3$ & $8.4 \mathrm{E}-2$ & \begin{tabular}{|l|}
$3 \mathrm{E}-3$ \\
\end{tabular} & $8.1 \mathrm{E}-2$ & $2 \mathbf{E}-2$ & 56.51 & 9.53 & 56.91 & 10.70 & $52 . \overline{78}$ & 9.84 & 54.12 & 9.32 & 45.35 & 12.10 & 54.47 & 6,06 & 5950 & 9.7 & 37.94 \\
\hline $\mathrm{Na} 2 \mathrm{O}$ & $1.0 \mathrm{E}+1$ & $0 \mathrm{E}+0$ & $1.4 \mathrm{E}+1$ & $\overline{1 E}+0$ & 38.81 & 7.01 & 39.38 & 9.92 & 33.56 & 6.14 & $35 . \overline{45}$ & 5.15 & 23.11 & 9.15 & 35.94 & 4.06 & 590873 & 960.9 & 27.36 \\
\hline $\mathrm{NiO}$ & $7.2 \mathrm{E}-4$ & $0 \overline{\mathrm{E}}+0$ & $4,5 \mathrm{E}-3$ & $4 \mathrm{E}-3$ & -185.12 & 278.31 & -182.48 & 277.71 & -209.58 & 301.46 & -200.80 & 292.57 & -258.25 & 349.89 & -198.50 & 182.24 & ND & & \\
\hline$\overline{\mathrm{P2O} 5}$ & $1.0 \mathrm{E}-1$ & 5E-3 & $1.6 \mathrm{E}-1$ & SE-2 & 29.70 & 22.74 & 30.35 & 23.95 & 23.66 & 24.14 & 25.83 & 23.20 & 11.66 & 28.71 & 26.40 & 14.70 & 2040 & 3.3 & 16.26 \\
\hline SO3 & 1.2E-1 & $0 \mathbf{E}+0$ & $1.3 \mathrm{E}-1$ & $4 \mathrm{E}-2$ & 54.69 & 13.37 & 55.11 & 14.25 & 50.80 & 14.13 & 52.20 & 13.55 & 43.07 & 16.90 & 52.56 & 8.62 & 8207 & 13.3 & 58.78 \\
\hline SiO2 & $3.0 \mathrm{E}+1$ & $4 E-1$ & $6.1 \mathrm{E}+1$ & $3 E+0$ & 7.90 & 9.03 & 8.75 & 13.92 & 0.00 & 7.08 & 2.84 & 5.17 & -15.72 & 11.93 & 3.58 & 4.93 & 47079 & 76.6 & 1.20 \\
\hline Sro & $5.9 \mathrm{E}-2$ & $1 \mathrm{E}-3$ & $1.0 \mathrm{E}-1$ & $9 \mathrm{E}-3$ & 20.41 & 9.82 & 21.15 & 13.40 & 13.59 & 8.91 & 16.04 & 7.72 & 0.00 & 12.75 & 16.68 & 5.79 & 2742.6 & 4.5 & 19.83 \\
\hline TiO2 & $7.2 \mathrm{E}-2$ & $0 \mathrm{E}+0$ & $1.7 \mathrm{E}-1$ & $5 \mathrm{E}-2$ & -4.54 & 31.82 & -3.58 & 33.77 & -13.51 & 33.69 & -10.29 & $32 . \overline{32}$ & -31.36 & 40.21 & -9.45 & 20.54 & ND & & 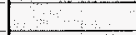 \\
\hline $\mathrm{ZrO} 2$ & $2.6 \mathrm{E}-2$ & $8 \mathrm{E}-4$ & $5.6 \mathrm{E}-2$ & $8 \mathrm{E}-3$ & 2.32 & 16.51 & 3.22 & 19.88 & -6.06 & 16.42 & -3.05 & 15.21 & -22.73 & 21.11 & -2.26 & 10.25 & ND & & \\
\hline Cl- & $3.1 \mathrm{E}-1$ & $0 \mathrm{E}+0$ & $4.5 \mathrm{E}-2$ & $6 \mathrm{E}-3$ & 93.42 & 1.08 & 93.49 & 1.32 & 92.86 & 1.07 & 93.06 & 0.99 & 91.74 & 1.39 & 93.12 & 0.67 & 40901 & 66.5 & 47.30 \\
\hline F. & $1.6 \mathrm{E}-1$ & 5E-10 & $2.0 \mathrm{E}-2$ & $3 \mathrm{E}-2$ & 94.35 & 7.64 & 94.40 & 7.60 & 93.87 & 8.28 & 94.04 & 8.04 & 92.90 & 9.60 & 94.09 & 5.01 & 3400 & 5.5 & 7.60 \\
\hline I- & $8.2 \mathrm{E}-2$ & $0 \mathrm{E}+0$ & $7.5 \mathrm{E}-3$ & $0 \mathrm{E}+0$ & 95.88 & N/A & 95.92 & N/A & 95.52 & $\mathrm{~N} / \mathrm{A}$ & 95.65 & $\mathrm{~N} / \mathrm{A}$ & 94.82 & NA & 95.69 & $\mathrm{NA}$ & $<222$ & 0.4 & 0.97 \\
\hline & & & & & & & & & & Total $\mathrm{m}$ & of oxid & nalyte & ried o & $(\mathrm{lbs})=$ & & & & & 6.15 \\
\hline & & & & & & & & & & Total can & over of & ieoretical & glass mas & (Metho & d 5 Sample & & & & $13.3 \%$ \\
\hline
\end{tabular}




\section{Table 6. Total Carryover Estimates from Cummulative Glass and Feed Values}

Slurry feed rate reported $=$

Total amount of feed reported in figure 5-2 (oxide basis) $=$

Conversion of reported total feed oxides to slurry using B\&W's conversion $(56.47 \%$ of slurry $=$ oxides $)=$

Calculated total feeding time using $B \& W ' s$ feed rate and total $=$

Combined "loss on drying" and "loss on ignition" from feed analytical values =

Revised \%oxides-in-slurry value using loss on drying \& ignition value =

Revised calculation of total oxides fed using using new slurry-to-oxides

conversion $=$

Total amount of glass generated, reported in Figure 5-2 (note that this material was measured wet, after fritting, and $B \& W$ did not correct for water in their report $)=$

Estimated amount of residual water in glass frit (from Vortec glass cullet measurements) $=$

Revised total amount of dry glass generated (correcting for residual water) =

Revised total volatility/entrainment estimate using loss drying \& ignition data and correcting for residual water in glass cullet =
$100 \mathrm{lb}$ slurry/hr

$753 \mathrm{~kg}$ oxides

$1333 \mathrm{~kg}$ slurry

$29.4 \mathrm{hrs}$

$53.7 \%$

$46.3 \%$

$617 \mathrm{~kg}$ oxides

577 kg

$23 \%$

$444 \mathrm{~kg}$

$28.0 \%$

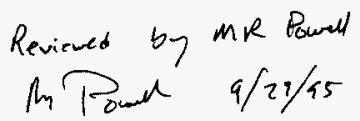


WHC-SD-WM-ER-517

Revision 0
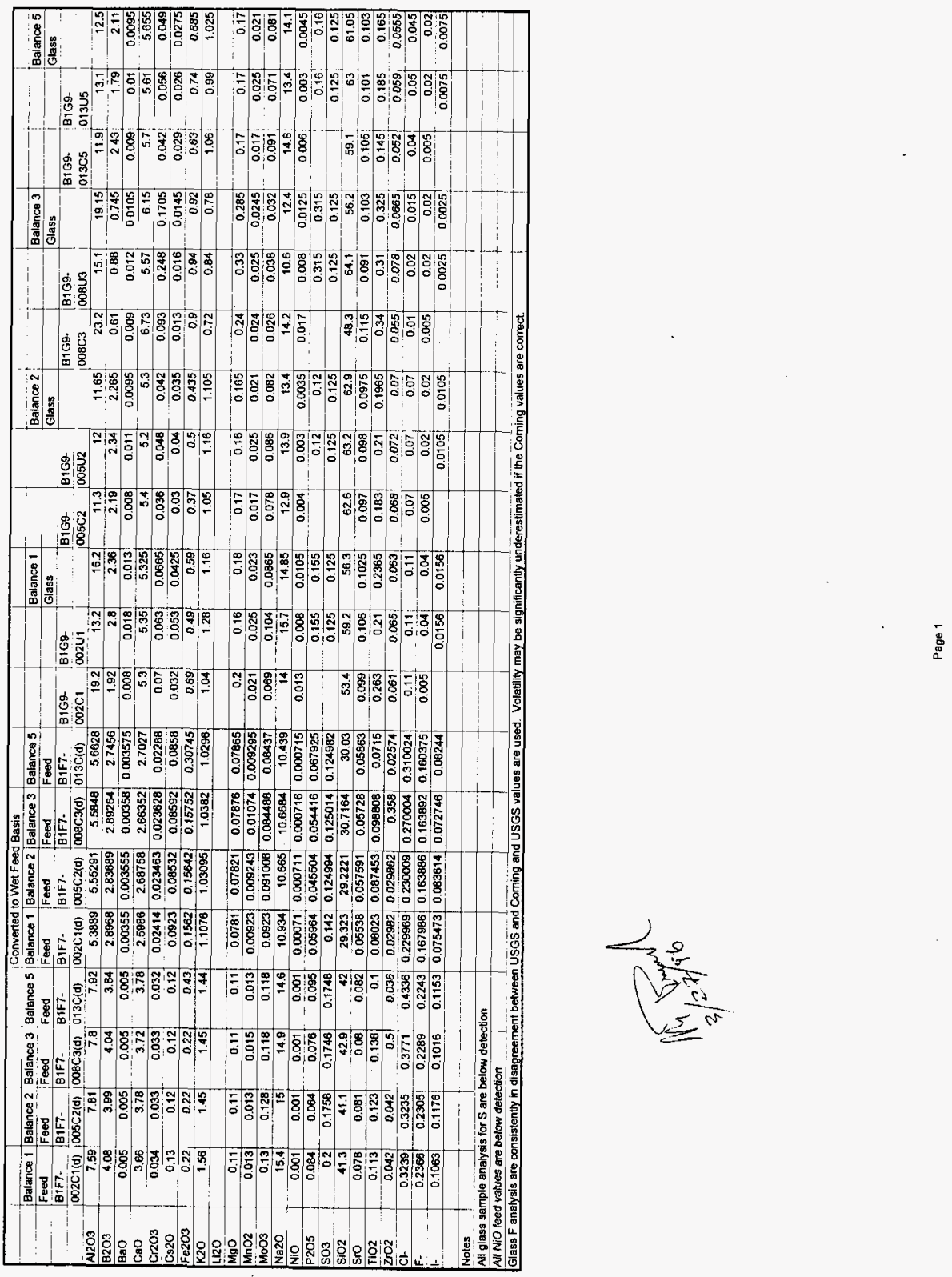


\section{Summary Tie Component Results}

\begin{tabular}{|c|c|c|c|c|c|c|c|c|c|c|}
\hline \multirow{2}{*}{\multicolumn{2}{|c|}{\begin{tabular}{|l|} 
Sample No. \\
Date \\
Time \\
\end{tabular}}} & \multirow{2}{*}{\multicolumn{2}{|c|}{$\begin{array}{l}\text { Balance \#1 } \\
\text { \% Volatility (Ave, } \\
\text { St,Si,Ca) Based On } \\
\text { Feed \& Glass } \\
\text { Ave. Stdv }\end{array}$}} & \multirow{2}{*}{\multicolumn{2}{|c|}{$\begin{array}{l}\text { Balance \#2 } \\
\text { \% Volatility (Ave, } \\
\text { Si,Ca, Sr) Based On } \\
\text { Feed \& Glass } \\
\text { Ave. Stdv }\end{array}$}} & \multicolumn{2}{|c|}{$\begin{array}{l}\text { Balance \#3 } \\
\% \text { Volatility (Ave, } \\
\text { Si,Ca,Sr) Based On } \\
\text { Feed \& Glass }\end{array}$} & \multicolumn{2}{|c|}{$\begin{array}{l}\text { Balance \#5 } \\
\% \text { Volatility (Ave, } \\
\mathrm{Al}, \mathrm{Si}, \mathrm{Ca} \text { ) Based On } \\
\text { Feed \& Glass }\end{array}$} & \multirow[t]{2}{*}{$\begin{array}{l}\text { Overall } \\
\text { Average }\end{array}$} \\
\hline & & & & & & Ave. & Stdv & Ave. & Stdv & \\
\hline & \begin{tabular}{|l|}
$\mathrm{A} 12 \mathrm{O} 3$ \\
\end{tabular} & -55.23184 & 25.338177 & $7 \quad-9.260139$ & 4.8753003 & -75.53531 & 37.500089 & -4.692535 & 6.961635 & -36.17995 \\
\hline & $\mathrm{B} 2 \mathrm{O} 3$ & 57.93127 & 7.0082752 & 58.420222 & 0.8988644 & 86.815453 & 1.5227093 & 63.551294 & 1.4793541 & 66.67956 \\
\hline & $\mathrm{BaO}$ & -89.09538 & 75.231292 & $2-39.16834$ & 27.228643 & -50.14456 & 29.139483 & -26.03306 & 14.443024 & $+\quad-51.11033$ \\
\hline & $\mathrm{CaO}$ & -5.81472 & 3.8423025 & -2.700114 & 3.1278143 & -18.20136 & 16.667274 & 0.7634419 & 4.1680208 & $3-6.488189$ \\
\hline & $\mathrm{Cr} 2$ & -42.24935 & 12.93854 & 6.777186 & 12.748735 & -269.4033 & 150.64078 & -1.572697 & 19.333547 & -76.61204 \\
\hline & Cs2 & 76.223215 & 4.7899457 & 78.636438 & 2.953796 & & 1.32 & 84 & 55057 & \\
\hline & $\mathrm{Fe} 2$ & -95.04593 & 31.652346 & -44.8283 & 19.405396 & -198.9892 & 36.332091 & -5.670314 & 10.450208 & $3-86.13343$ \\
\hline & $\mathrm{K} 2 \mathrm{O}$ & 45.919469 & 5.3332395 & 44.181118 & 2.6793105 & 61.539325 & 5.1571238 & 52.783667 & 2.3868114 & 451.1 \\
\hline & $\mathrm{Li} 2 \mathrm{O}$ & $\mathrm{NA}$ & NA & NA & $\mathrm{N} / \mathrm{A}$ & $\mathrm{N} / \mathrm{A}$ & $\mathrm{N} / \mathrm{A}$ & $\mathrm{N} / \mathrm{A}$ & N/A & NA \\
\hline & $\mathrm{MgO}$ & -19.01108 & 16.901022 & -9.869745 & 4.3082859 & -85.24329 & 34.112609 & -2 & 7.76 & $4-29$ \\
\hline & $\mathrm{MnO} 2$ & -28.67437 & 12.222702 & -18.32126 & 21.094939 & & 14.108605 & -7.153615 & 22.304434 & \\
\hline & $\mathrm{MoO} 3$ & 51.607249 & 9.1709523 & 53.076463 & 2.4836488 & 80.610872 & 4.3552673 & 54.4662 & 6.0556406 & 59.940196 \\
\hline & $\mathrm{Na}_{2}$ & 29.868474 & 4.1904643 & 34.566463 & 2.4543263 & 40.4988 & 9.8750688 & 35.938495 & 4.0551422 & 35.2 \\
\hline & $\mathrm{NiO}$ & -663.6544 & 253.39761 & -156.3627 & 159.32385 & -793.7176 & 306.00693 & -198.4994 & 182.23868 & \\
\hline & $\mathbf{P} 205$ & 25.890506 & 14.350288 & 40.651556 & 10.790597 & -53.1389 & 33.415096 & 26.396538 & 14.700844 & 9.9499262 \\
\hline & $\mathrm{SO} 3$ & - & 7.6004166 & & 8.6206262 & 48.813424 & 10.276358 & 52.564938 & & 50.960475 \\
\hline & $\mathrm{SiO} 2$ & 0.856122 & 5.731869 & -12.09744 & 2.7894269 & 6.3367274 & 15.566048 & 3.5799702 & 4.9342398 & $3-0.331154$ \\
\hline & $\mathrm{SrO}$ & 4.4266472 & 4.9408672 & 11.832921 & 2.9614706 & 7.9470858 & 15.896127 & & 5.7865908 & \\
\hline & $\mathrm{TiO2}$ & -52.21598 & 18.550739 & -17.01574 & 9.264255 & -68.38158 & 20.477714 & -9.449764 & 20.537913 & -36.76577 \\
\hline & $\mathrm{ZrO2}$ & -9.093486 & 9.2682836 & -22.07749 & 6.8992571 & 90.490845 & 2. & -2.263668 & 10.254927 & \\
\hline & $\mathrm{Cl}-$ & 75.300435 & 1.2662675 & 84.150681 & 0.50 & 97.15603 & & & & \\
\hline & & 87.704313 & 9.827274 & 93.644556 & 4.9780117 & 93.752957 & 4.9484233 & 94.085315 & 5.0050353 & 92.2 \\
\hline & & 89.326696 & & 93.460134 & & 98.240713 & & 95.685178 & & 94.17818 \\
\hline
\end{tabular}

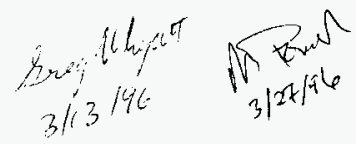


Revision 0

Battelle

CALCULATION WORKSHEET

Calc. No.

Pacifıc Northwest Laboratories

Page 1 of 3

Sperial Estimater $B+w$

Hess Balanes 1- Eeed

- No $\mathrm{SO}_{3}$ data in selected sumplo so we slury and 29 wo lossondryin]

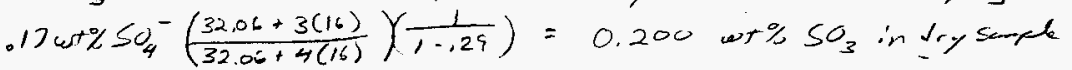

$+50$

- Ho el data, use slurry date .23 wt b amb loss ondry with caocherk check: $.230+\%\left(\frac{3.66}{2.60}\right)=.3165 \mathrm{w} \%$ ol in Jry sarpel

uce loceon dry basis $.23(\sqrt{. .29})=.3239$ ce ind.y sungle

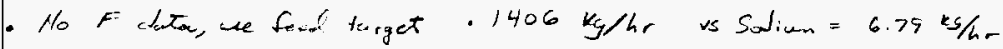

$$
\left(15.4 N_{\pi_{2}} \mathrm{O} \therefore \text { fod }\right)\left(\frac{.1406 \mathrm{~F}}{6.79 \mathrm{~kg} \mathrm{~N}_{4}\left(\frac{46+16}{46}\right)}\right)=.2366 \omega+\mathrm{x} F \text { in dry Sample }
$$

- No $I^{-}$.Jta, use actiration andlysis BIF 7-002U $=0.0904$ wt 2 Sal... $=13.1 .1 \% \mathrm{Na}_{2} \mathrm{O}$

$$
15.4\left(\frac{.0904}{13,1}\right)=.1063 w+\% I \text { in drial sapple }
$$

- Glowed $\mathrm{PO}_{5}$ only I dutu point so we that

SQ neithon sample has dute except Netection limit, wae thet

$I^{-}$I data point une thet

Hass Balcuar $=2$ - Enel

- No 503 Jerzie, une slurry

$$
.15 \mathrm{SO}_{4}=\left(.8334 \frac{50_{3}}{504}\right)\left(\frac{1}{1 . .289}\right)=.1758 \ldots+250_{3}
$$

roel Natew, we slury $.23\left(\frac{1}{1-.289}\right)=.3235$ wty el

No F Late we target $15.0\left(1.536 \times 10^{-2}\right)=.2305 \mathrm{wt} \% \mathrm{~F}$

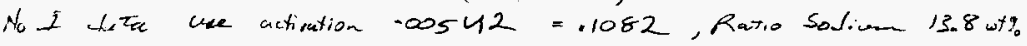

$$
15.0\left(\frac{.1082}{13.8}\right)=.1176 \text { wt\% }
$$

Glues same as mass bulanes.

BEST AMMLABIE COPY

Sitle

Project

LLw yitrification Prepared by:

Greq Whyatt

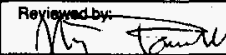

$\frac{3427 / 196}{3 / 27}$ 
Revision 0

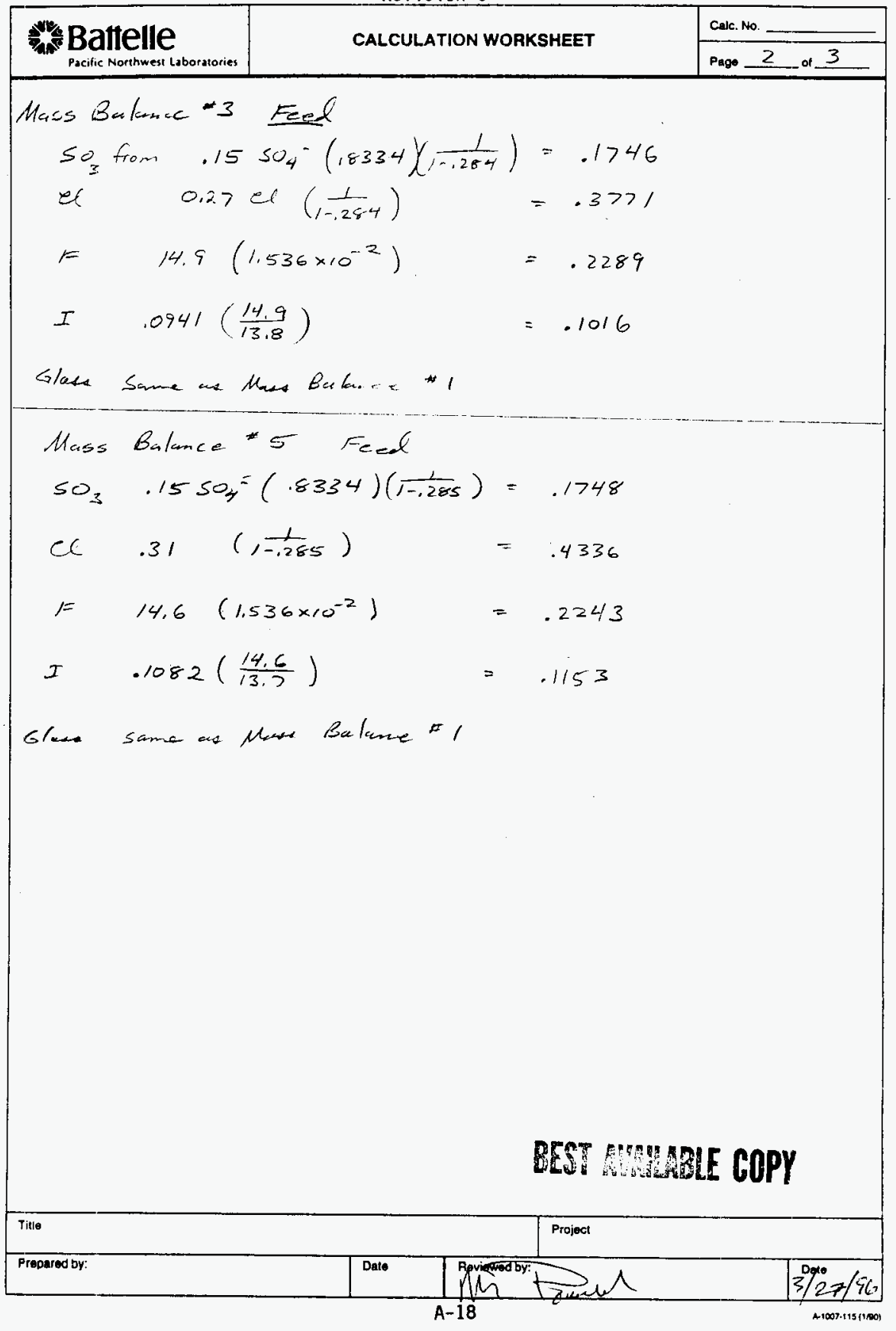


Revisione

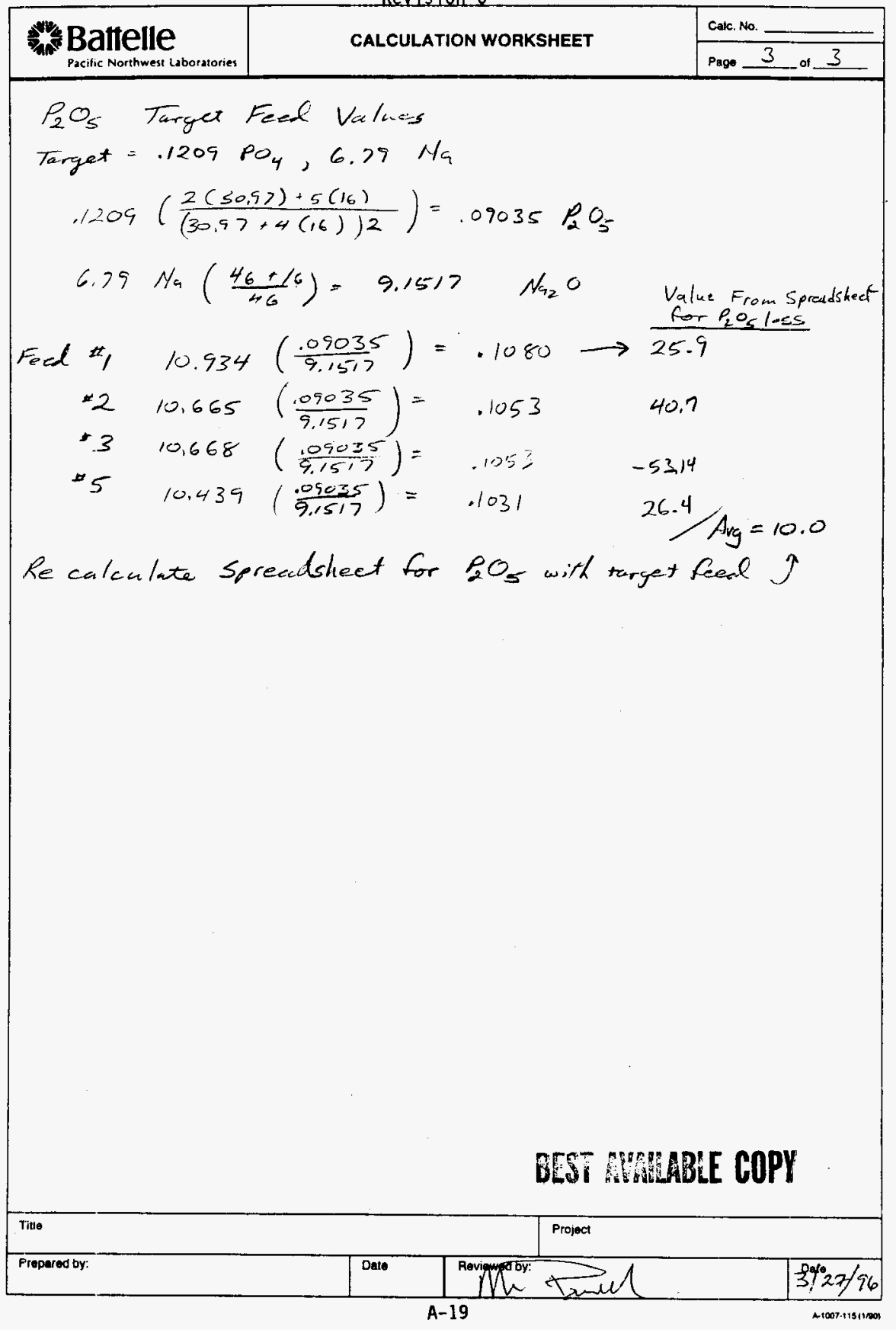


WHC-SD-WM-ER-517

Revision 0

This page intentionally left blank. 
WHC-SD-WM-ER-517

Revision 0

APPENDIX B

REVIEW OF WESTINGHOUSE SCIENCE AND TECHNOLOGY CENTER TEST REPORT 
WHC-SD-WM-ER-517

Revision 0

This page intentionally left blank. 
WHC-SD-WM-ER-517

Revision 0

\section{Westinghouse Science and Technology Center Melter (WSTC) Entrainment and Volatility Estimates}

The WSTC analytical data were examined and estimates made of the relative volatility and entrainment for each oxide analyte. These analyses and the results are described below. The volatility estimates were made by comparing the melter feed and glass product compositions. Assuming uniform entrainment of feed and zero volatility for a known low volatility component, the fractional volatility of each glass oxide species can be estimated. The entrainment of melter feed was estimated based on the observed average aerosol composition and the total mass of oxides known to have escaped to the off-gas system.

\section{Entrainment}

Feed entrainment during the 24-hour demonstration run test is estimated to have been between $2 \%$ and $3 \%$ of the feed oxides. Lower entrainment estimates based on aerosol collection rates are given in the vendor-prepared test report, but these estimates are suspect because of problems with the aerosol sampling procedures and test conditions. These problems include large uncertainties in the off-gas flow rate and the accumulation of solids upstream of the aerosol collection point. Further, the reported aerosol flow rates are much too low to account for the more than $1000 \mathrm{~kg}$ of glass mass that was missing from the glass product.

The $2 \%$ to $3 \%$ entrainment estimate was developed as follows. Based on the feed analyses and the known total mass of feed delivered to the melter, a total of $8383 \mathrm{~kg}$ of glass should have been produced during the 24-h demonstration test assuming no entrainment or volatility losses (p. 6-9 of McLaughlin et al.). Only $7272 \mathrm{~kg}$ of glass were recovered during the test (p. 6-6, ibid.). Therefore, a total of $1011 \mathrm{~kg}$ of glass must have been directed to the melter off-gas system. Assuming that the aerosol samples collected during the test accurately reflect the composition of the aerosols (but not necessarily the aerosol flow rate), the rate of feed entrainment can be estimated using the aerosol sample concentrations of non-volatile oxides like $\mathrm{SiO}_{2}, \mathrm{CaO}$, and $\mathrm{Al}_{2} \mathrm{O}_{3}$. Using this approach (see attached calculation worksheet), the feed entrainment is estimated by $\mathrm{SiO}_{2}$ concentrations to be $1.2 \%$, $\mathrm{CaO}$ gives $3.3 \%$, and $\mathrm{Al}_{2} \mathrm{O}_{3}$ gives $2.1 \%$. Because $\mathrm{SiO}_{2}$ analyses have proven to be somewhat unreliable in the past, the entrainment estimates given by $\mathrm{Al}_{2} \mathrm{O}_{3}$ and $\mathrm{CaO}$ are probably more accurate.

\section{Volatility}

Fractional volatility estimates were generated for each of the glass oxide analytes. To make these calculations, it is necessary to select a tie-component, which is assumed to have zero volatility. Experience has shown that $\mathrm{SiO}_{2}, \mathrm{CaO}$, and $\mathrm{Al}_{2} \mathrm{O}_{3}$ are generally good low volatility tie-components. The volatility calculations were made independently for each tiecomponent for each of the four snapshot samples taken during the 24-h demonstration test. The results are given in the attached spreadsheets. 
The fractional volatilities calculated using $\mathrm{CaO}$ and $\mathrm{Al}_{2} \mathrm{O}_{3}$ as the tie-component are judged most reliable. As noted earlier, $\mathrm{SiO}_{2}$ analyses are often in error because of analytical difficulties. There is no compelling reason to select $\mathrm{CaO}$ as the tie component rather than $\mathrm{Al}_{2} \mathrm{O}_{3}$ or vice versa, so reported in Table 1 for each glass oxide is the average of the CaO-tie volatility and the $\mathrm{Al}_{2} \mathrm{O}_{3}$-tie volatility.

The volatility data for certain glass oxides require some further explanation. The fractional volatilities for $\mathrm{Na}_{2} \mathrm{O}, \mathrm{K}_{2} \mathrm{O}$, and $\mathrm{F}$ show a significant increase over the course of the test. This is consistent with the hypothesis that the glassformer addition rate decreased during the test, thereby increasing the waste loading. As the waste loading is increased, it is reasonable to expect a concomitant increase in the fractional volatilities for the waste-borne volatile components. The effects of the changing glassformer addition rate are explored in detail in the report prepared by McLaughlin et al. (1995).

The $\mathrm{SO}_{3}$ volatilities vary between -0.4 and 0.43 . This is believed to be the result of analytical errors primarily in the analyses of the third feed sample. The $\mathrm{SO}_{4}^{2-}$ concentrations for the first, second, and forth snapshot analyses are $0.17 \mathrm{wt} \%, 0.18 \mathrm{wt} \%$, and $0.14 \mathrm{wt} \%$. The third snapshot, however, was reported to contain only $0.091 \mathrm{wt} \%$. In view of this, it is expected that the best estimate for $\mathrm{SO}_{3}$ fractional volatility is approximately 0.3 to 0.4 .

It is not clear why $\mathrm{MgO}, \mathrm{Fe}_{2} \mathrm{O}_{3}$ and $\mathrm{TiO}_{2}$ are found to have negative volatilities (which imply a net gain of the species as the feed passes through the melter). The concentrations of $\mathrm{MgO}$ and $\mathrm{TiO}_{2}$ are both quite low in the feed and the glass samples, so it may be appropriate to attribute these data to analytical uncertainties. The ferric oxide concentrations, however, are well within the measurable range, so it is unlikely that the negative $\mathrm{Fe}_{2} \mathrm{O}_{3}$ volatilities are due to random analytical uncertainties alone.

\section{Reference:}

McLaughlin, D. F., W. C. Yang, W. R. Gass, S. V. Dighe, N. D'Amico, R. L. Swensrud, and M. F. Darr. 1995. Evaluation of Plasma Melter Technology for Vitrification of HighSodium Content Low-Level Radioactive Liquid Wastes -- Demonstration Test \#4 Final Test Report. 95-9TD2-VITNA-R2. Distributed by Westinghouse Hanford as document WHC-SDWM-VI-016, Rev. A. April 28, 1995. 
WHC-SD-WM-ER-517

Revision 0

Table 1. Fractional Volatility Calculations with $\mathrm{CaO}$ and $\mathrm{Al} 2 \mathrm{O} 3$ Tie Components

\begin{tabular}{||c|c|c|c|c|c||}
\hline \multicolumn{6}{|c|}{ Estimate of Feed Oxide Volatilities (avg. of CaO and $\mathrm{Al}_{2} \mathrm{O}_{3}$ tie values) } \\
\hline Oxide & Snap \#1 & Snap \#2 & Snap \#3 & Snap \#4 & Avg. \\
\hline $\mathrm{Al}_{2} \mathrm{O}_{3}$ & -0.051 & 0.010 & -0.054 & -0.0020 & -0.025 \\
\hline $\mathrm{B}_{2} \mathrm{O}_{3}$ & 0.31 & 0.14 & 0.22 & 0.19 & 0.22 \\
\hline $\mathrm{BaO}$ & 0.30 & 0.0074 & -0.12 & 0.039 & 0.058 \\
\hline $\mathrm{CaO}$ & 0.047 & -0.010 & 0.049 & 0.0019 & 0.022 \\
\hline $\mathrm{Cr}_{2} \mathrm{O}_{3}$ & 0.13 & 0.24 & -0.011 & 0.10 & 0.12 \\
\hline $\mathrm{Cs}_{2} \mathrm{O}$ & 0.83 & 0.83 & 0.81 & 0.88 & 0.84 \\
\hline $\mathrm{Fe}_{2} \mathrm{O}_{3}$ & -0.28 & -0.13 & -0.20 & -0.66 & -0.32 \\
\hline $\mathrm{K}_{2} \mathrm{O}$ & 0.43 & 0.48 & 0.43 & 0.59 & 0.48 \\
\hline $\mathrm{Li}_{2} \mathrm{O}$ & 0.017 & 0.045 & 0.072 & -0.024 & 0.028 \\
\hline $\mathrm{MgO}_{2}$ & -0.14 & -0.039 & -0.10 & -0.23 & -0.13 \\
\hline $\mathrm{MnO}_{2}$ & 0.30 & 0.42 & 0.25 & -0.072 & 0.22 \\
\hline $\mathrm{MoO}_{3}$ & 0.15 & 0.22 & 0.18 & 0.39 & 0.24 \\
\hline $\mathrm{Na}_{2} \mathrm{O}$ & 0.026 & 0.17 & 0.098 & 0.31 & 0.15 \\
\hline $\mathrm{NiO}^{2}$ & 0.69 & 0.69 & 0.69 & 0.73 & 0.70 \\
\hline $\mathrm{P}_{2} \mathrm{O}_{5}$ & 0.38 & 0.56 & 0.41 & 0.36 & 0.43 \\
\hline $\mathrm{SO}_{3}$ & 0.37 & 0.43 & -0.40 & 0.23 & 0.34 \\
\hline $\mathrm{SiO}_{2}$ & 0.14 & 0.092 & 0.12 & 0.092 & 0.11 \\
\hline $\mathrm{SrO}^{(\mathrm{a})}$ & -0.12 & 0.042 & -0.088 & 0.16 & -0.0005 \\
\hline $\mathrm{TiO}_{2}$ & -1.2 & -1.5 & -3.4 & -2.0 & -2.0 \\
\hline $\mathrm{ZrO}_{2}$ & 0.0047 & -0.0082 & 0.017 & -0.038 & -0.0061 \\
\hline $\mathrm{Cl}^{-}$ & 0.88 & 0.87 & 0.85 & 0.90 & 0.88 \\
\hline $\mathrm{F}$ & 0.42 & 0.44 & 0.58 & 0.65 & 0.52 \\
\hline $\mathrm{Th}$ & $\# 3$ & & & & \\
\hline
\end{tabular}

(a) The snapshot \#3 feed result for sulfate is believed to be in error so the average sulfur loss is calculated based on snapshots 1,2 and 4 only. 


\section{WHC-SD-WM-ER-517}

Revision 0

Traceability Aid For Analytical Samples

Sample analysis results are located in WHC-SD-WM-DP-131, Rev.0 "Low-Level Waste

Vitrification Phase 1 Test Sample Analyses Data" under the following sample numbers.

Snapshot \#1 was based on the following samples:

glass W1G07-404C

feed W1F12-401C1(d)

Snapshot \#2 was based on the following samples:

glass W1G07-404C2

feed W1F12-401C2(d)

Snapshot \# was based on the following samples:

glass W1G07-404C3

feed W1F12-401C3(d)

Snapshot \# was based on the following samples:

glass W1G07-404C4

feed W1F12-401C4(d) 


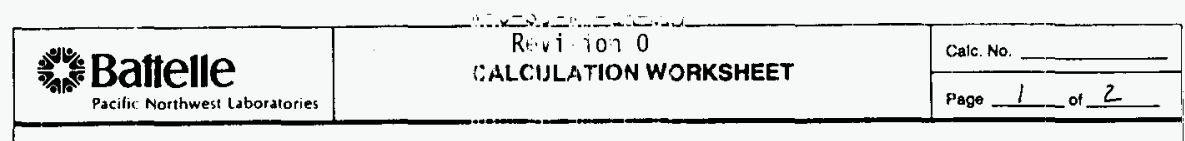

Estimate WSTC entrainment dipping 24 -h run

Data: from Rev. A of "Nuluoticn of Plasma Melter Technology for Vitrification of High-Sodium Content Low -Level Radioactive Liquid Wastes - Demonstration Test 4 final Test Report 4/26/15 p. 6-6: Total glass mass produced $=7372 \mathrm{~kg}$

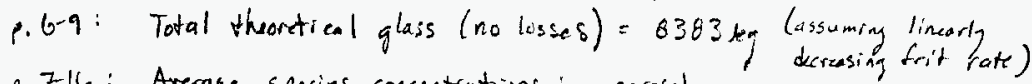

0.716: Average species concentrations in aerosol Samples (from Tab 6 7.6 )

$$
\begin{aligned}
& \mathrm{SiO}_{2}=4,04 \% \\
& \mathrm{CaO}_{2}=1.11 \% \\
& \mathrm{Al}_{2} \mathrm{O}_{3}=2.92 \%
\end{aligned}
$$

p. 5-8: Classtormas analysis (Fable 5.3)

$$
\begin{aligned}
& \mathrm{SiO}_{2}=55.43 \pm 1.35 \% \\
& \mathrm{CaO}_{2}=5.61 \pm 0.27 \% \\
& \mathrm{Al}_{2} \mathrm{O}_{3}=17.97 \pm 1.02 \%
\end{aligned}
$$

p. 5-21: Simulant analysis (Table 5.11)

$$
\begin{aligned}
& \mathrm{SiO}_{2}=0 \\
& \mathrm{CaO}^{2}=0.46 \% / \mathrm{L} \\
& \mathrm{Al}_{2} \mathrm{O}_{3}=56.56 \% / \mathrm{L}
\end{aligned}
$$

p. 5.19: Average simulant flow rate $=3.755 \mathrm{~L} / \mathrm{min}$

p. 6-9: Average glessformer feed rate $\cong 3.95 \mathrm{ki} / \mathrm{min}$,

Calculations:

Total missing oxide mass $=8383.7372=1011 \mathrm{~kg}$

Estimated amount of $\mathrm{CaO}$ in missing mass $=(1011 \mathrm{~kg})(0.011 \%)=11.22 \mathrm{~kg}(.0$ -

$$
\begin{aligned}
\text { Ant of CoO fed to melt re } & =(10561)(3.95 \mathrm{kgm})(1540 \mathrm{~min})+\left(.00046 \frac{\mathrm{kg}}{\mathrm{L}}\right)(3.755 \mathrm{~mm})(154 \mathrm{amm}) \\
& =343.9 \mathrm{~kg} \mathrm{CaO} \mathrm{fed}
\end{aligned}
$$

$$
\therefore \quad \% \mathrm{CaO} \text { entrained }=\frac{11.22 \mathrm{~kg}}{3413.9 \mathrm{~kg}} \times 100 \%=3.26 \%
$$

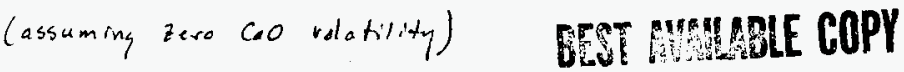

Title

WSTC entrainment estimate

Project

M.R. Powell N in towel

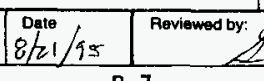
LLW bit.

B-7 
Battelle

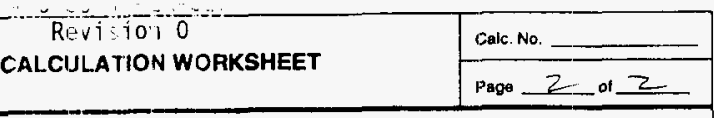

(Cont.):

Similarly for $\mathrm{SiO}_{2}$ and $\mathrm{H}_{2} \mathrm{O}_{3}$,

$$
\begin{aligned}
& \mathrm{SiO}_{2} \text { entrained }=(101(\mathrm{~kg})(.0404)=40.84 \mathrm{~kg} \\
& \mathrm{SiO}_{2} \text { fed }=(.5543)(3.95)(1540)=3372 \mathrm{~kg} \\
& \% \mathrm{SiO}_{2} \text { Entrained }=\frac{40.84}{33 \% 2} \mathrm{Nos}=1.21 \% \\
& \mathrm{H}_{2} \mathrm{O}_{3} \text { entrained }=(1011 \mathrm{~kg})(.0292)=29.5 \mathrm{kq} \\
& \mathrm{Al}_{2} \mathrm{O}_{3} \mathrm{fed}=(.1797)(3.95)(1540)+(.05698)(3.755)(1540)=1422.6 \mathrm{~kg} \\
& \% \mathrm{Al}_{2} \mathrm{O}_{3} \text { Entrained }=\frac{29.5}{1422.6}=2.08 \%
\end{aligned}
$$
Note that some significant problems have been noted with some likely that the $\mathrm{CaO}^{\circ}$ and $\mathrm{Ki}_{2} \mathrm{O}_{3}$ entrainment estimates ane

PEST NAMABLE COPY

WSTC entrainment estimate

Project

Prepared by: R. Powell Ar rand

Reviewed by:

B-8 


\begin{tabular}{|c|c|c|c|c|c|c|}
\hline \multicolumn{7}{|c|}{ WSTC Averaging of Volatile Losses from Spreadsheets (non-linked, copy-paste special) } \\
\hline oxide & snap 1 & snap 2 & $\operatorname{snap} 3$ & snap 4 & Tavg & \\
\hline $\mathrm{Al} 2 \mathrm{O} 3$ & -005142 & 0009849 & -0.05445 & -0.00195 & -0.02449 & \\
\hline $\mathrm{B} 2 \mathrm{O} 3$ & 0.308476 & 0.143535 & 0.219128 & 0.189858 & $0.2 \overline{15249}$ & \\
\hline $\mathrm{BaO}$ & 0.301708 & 0.0074 & -0.11608 & 0.038783 & 0.057953 & \\
\hline $\mathrm{CaO}$ & $0.046 \overline{629}$ & -0.01005 & 0.049103 & 0.00194 & 0.021906 & \\
\hline $\mathrm{C} 2 \mathrm{2O}$ & 0.130415 & 0.242449 & -0.01051 & 0.096615 & 0.114742 & \\
\hline $\mathrm{Cs} 2 \mathrm{O}$ & 0.833688 & 0.829904 & 0.811631 & 0.877869 & 0.838273 & \\
\hline $\mathrm{Fe} 2 \mathrm{O} 3$ & -0.27871 & -0.13047 & -0.20136 & -0.66357 & -0.31853 & \\
\hline $\mathrm{K} 2 \mathrm{O}$ & 0.433001 & 0.477308 & 0.429249 & 0.590225 & 0.482445 & \\
\hline $\mathrm{Li} 2 \mathrm{O}$ & 0.01725 & 0.045006 & 0.07178 & -0.02358 & 0.027613 & \\
\hline $\mathrm{MgO}$ & -0.14202 & -0.03947 & -0.10176 & -0.23173 & -0.12874 & \\
\hline $\mathrm{MnO} 2$ & 0.298794 & 0.416997 & 0.246525 & -0.0716 & 0.222679 & \\
\hline $\mathrm{MoO3}$ & 0.154464 & 0.215962 & 0.184925 & 0.387656 & 0.235752 & \\
\hline $\mathrm{Na} 2 \mathrm{O}$ & 0.025903 & 0.167959 & 0.097619 & 0.313914 & 0.151349 & \\
\hline $\mathrm{NiO}$ & 0.68552 & 0.686385 & 0.689746 & 0.7321 & $0.69843 \overline{7}$ & \\
\hline$P 205$ & 0.380867 & 0.561919 & 0.408577 & 0.363737 & 0.428775 & \\
\hline $\mathrm{SO} 3$ & 0.371946 & 0.428462 & -0.40086 & 0.230171 & 0.157429 & \\
\hline $\mathrm{SiO} 2$ & 0.140758 & 0.091752 & 0.115251 & 0.092388 & 0.110037 & \\
\hline SrO & -0.11815 & 0.041731 & -0.08827 & 0.162811 & -0.00047 & \\
\hline $\mathrm{TiO} 2$. & -1.20136 & -1.50892 & -3.42113 & -2.01388 & -2.03632 & \\
\hline $\mathrm{ZrO2}$ & 0.00468 & -0.00822 & 0.017357 & -0.03837 & -0.00614 & \\
\hline $\mathrm{Cl}^{*}$ & 0.883326 & 0.868023 & 0.849433 & 0.900524 & 0.875327 & \\
\hline$F$ & 0.892696 & 0.883118 & 0.922849 & 0.941508 & 0.910043 & \\
\hline
\end{tabular}

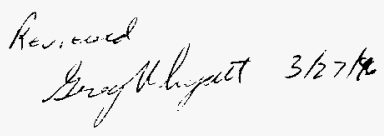




\begin{tabular}{|c|c|c|c|c|c|c|c|c|c|}
\hline \multicolumn{10}{|c|}{ MR Powell $8 / 19 / 95$} \\
\hline \multicolumn{10}{|c|}{ WSTC Data: Estimated Oxide Volatilities based on glass and feed compositions and tie components } \\
\hline & & & & & & & & & \\
\hline \multicolumn{10}{|c|}{ Snapshot \#1 taken 12/07/94 at 17:00 } \\
\hline & & & & Tie $=$ & Tie $=$ & Tie $=$ & Tie $=$ & & Avg of $\mathrm{Ca}$ \\
\hline & Average & Average & & $\mathrm{SiO} 2$ & $\mathrm{Fe} 2 \mathrm{O} 3$ & $\overline{\mathrm{CaO}}$ & $\mathrm{Al2O} 3$ & & and $\mathrm{Al} 2 \mathrm{O} 3$ \\
\hline & Glass & Feed & & $\mathrm{Vi}$ & $\mathrm{Vi}$ & $\mathrm{Vi}$ & Vi & & \\
\hline & & & & & & & & & \\
\hline $\mathrm{Al} 2 \mathrm{O} 3$ & 19.425 & 14.525 & & -0.22367 & 0.177743 & -0.10285 & 0 & & -0.05142 \\
\hline $\mathrm{B} 2 \mathrm{O} 3$ & $6.66 \overline{5}$ & 7.5775 & & 0.195192 & 0.4592 & 0.274654 & 0.342298 & & 0.308476 \\
\hline $\mathrm{BaO}$ & 1.0525 & 1.185 & & 0.187316 & 0.453907 & 0.267555 & 0.335861 & & 0.301708 \\
\hline $\mathrm{CaO}$ & 4.32 & 3.5625 & & -0.10955 & 0.254424 & 0 & 0.093257 & & 0.046629 \\
\hline $\mathrm{Cr} 2 \mathrm{O} 3$ & 0.0365 & 0.033 & & -0.01204 & 0.319949 & 0.087884 & 0.172946 & & 0.130415 \\
\hline $\mathrm{Cs} 2 \mathrm{O}$ & 0.0275 & 0.13 & & 0.806444 & 0.869937 & 0.825554 & 0.841823 & & 0.833688 \\
\hline $\mathrm{Fe} 2 \mathrm{O3}$ & 0.7075 & $0 . \overline{3} \overline{5}$ & & -0.48818 & 0 & -0.34125 & -0.21616 & & -0.27871 \\
\hline $\mathrm{K} 2 \mathrm{O}$ & 0.97 & 1.345 & & 0.340116 & 0.556583 & 0.405269 & 0.460732 & & 0.433001 \\
\hline $\mathrm{Li} 2 \mathrm{O}$ & 0.8375 & $0 . \overline{6}$ & & -0.14374 & 0.231449 & -0.03082 & 0.065315 & & 0.01725 \\
\hline $\mathrm{MgO}$ & 0.08425 & 0.058 & & -0.32911 & 0.10689 & -0.19788 & -0.08617 & & -0.14202 \\
\hline $\mathrm{MnO} 2$ & 0.00825 & 0.00925 & & 0.183925 & 0.451628 & 0.264499 & 0.33309 & & 0.298794 \\
\hline MoO3 & 0.1425 & 0.1325 & & 0.015951 & 0.338756 & 0.113109 & 0.195818 & & 0.154464 \\
\hline $\mathrm{Na} 2 \mathrm{O}$ & 19.7 & 15.9 & & -0.13367 & 0.238216 & -0.02174 & 0.073545 & & 0.025903 \\
\hline $\mathrm{NiO}$ & 0.004 & 0.01 & & 0.634003 & 0.754064 & 0.670139 & 0.700901 & & 0.68552 \\
\hline $\mathrm{P} 2 \mathrm{O} 5$ & 0.1575 & 0.2 & & 0.279443 & 0.515813 & 0.350586 & 0.411149 & & 0.380867 \\
\hline $\mathrm{SO} 3$ & 0.1525 & 0.1909 & & 0.26906 & 0.508836 & 0.341228 & 0.402664 & & 0.371946 \\
\hline $\mathrm{SiO} 2$ & 37.35 & 34.175 & & 0 & 0.328038 & 0.098734 & 0.182783 & & 0.140758 \\
\hline SrO & 0.16 & 0.1125 & & -0.30132 & 0.125559 & -0.17284 & -0.06346 & & -0.11815 \\
\hline TiO2 & 0.028 & 0.01 & & -1.56198 & -0.72155 & -1.30903 & -1.09369 & & -1.20136 \\
\hline $\mathrm{ZrO} 2$ & 2.1775 & 1.72 & & -0.15837 & 0.221618 & -0.044 & 0.05336 & & 0.00468 \\
\hline $\mathrm{Cl}$ & 0.052 & 0.3504 & & 0.864213 & 0.908756 & 0.87762 & 0.889033 & & 0.883326 \\
\hline$F^{*}$ & 0.04 & 0.293075 & & 0.875118 & 0.916084 & 0.887448 & 0.897944 & & 0.892696 \\
\hline & & 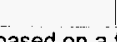 & & & & & & & 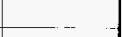 \\
\hline
\end{tabular}

$$
\text { Aevinged ithet } 3 / 27 / 46
$$




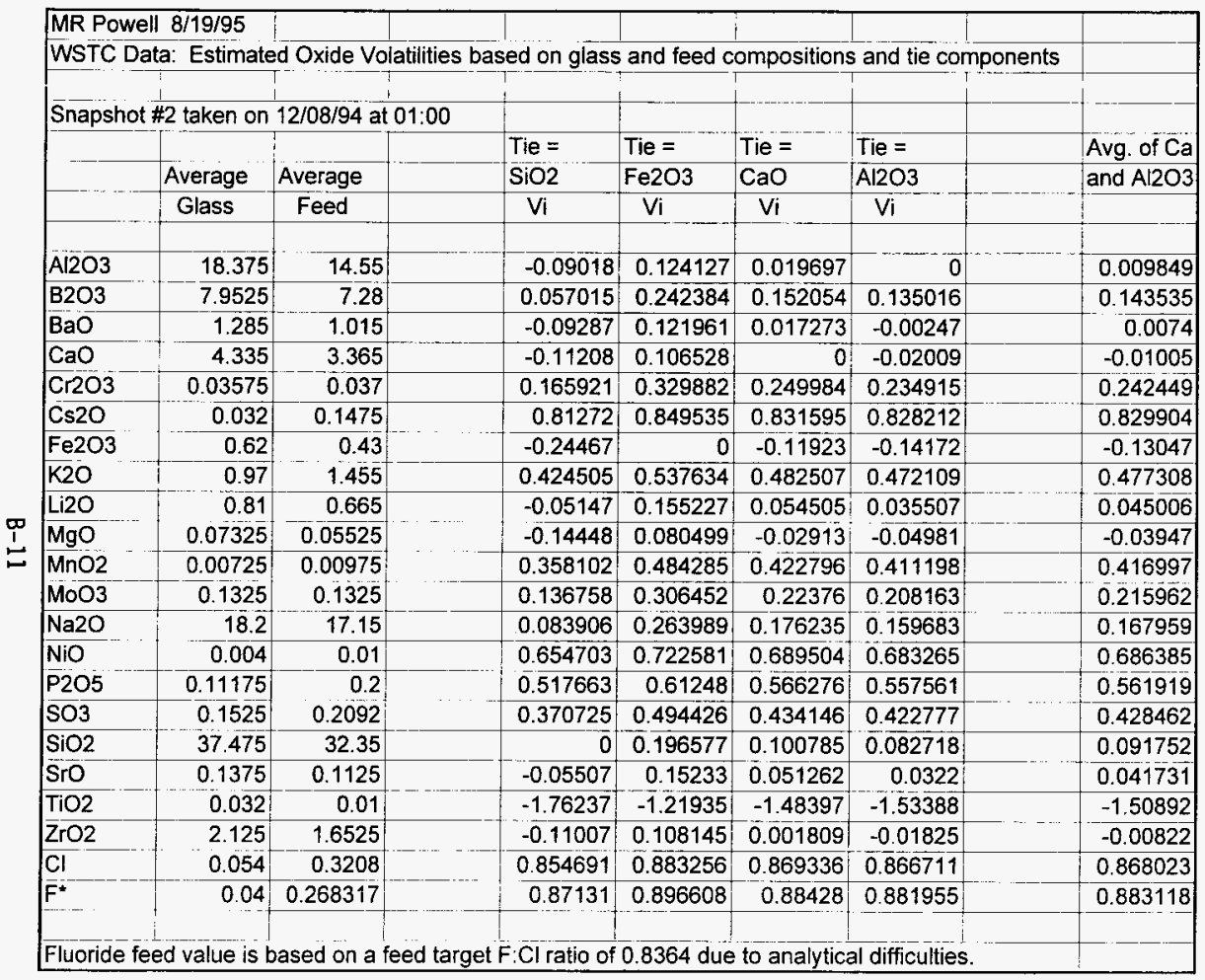

$$
\begin{aligned}
& \text { Kurvecued } \\
& \text { Sizy whight } 32>/ 16
\end{aligned}
$$




\begin{tabular}{|c|c|c|c|c|c|c|c|c|c|}
\hline \multicolumn{2}{|c|}{ MR Powell 8/19/95 } & & & & & & & & \\
\hline \multicolumn{9}{|c|}{ WSTC Data: Estimated Oxide Volatilities based on glass and feed compositions and tie components } & \\
\hline & & & & 1 & & & & & \\
\hline \multicolumn{10}{|c|}{ Snapshot $\# 3$ taken on $12 / 08 / 94$ at $09: 05$} \\
\hline \multirow{4}{*}{} & & & & Tie $=$ & Tie $=$ & $\mathrm{Tie}=$ & Tie $=$ & & Avg of $\mathrm{Ca}$ \\
\hline & Average & Average & & $\widehat{\mathrm{SiO} 2}$ & $\mathrm{Fe} 2 \mathrm{O} 3$ & $\mathrm{CaO}$ & $\mathrm{Al} 2 \mathrm{O} 3$ & & and $\mathrm{Al} 2 \mathrm{O} 3$ \\
\hline & Glass & Feed & & $\mathrm{Vi}$ & $\mathrm{Vi}$ & Vi & Vi & & \\
\hline & & & & & & & & & \\
\hline $\mathrm{Al} 2 \mathrm{O} 3$ & 17.775 & 13.075 & & -0.19181 & 0.122287 & -0.1089 & 0 & & -0.05445 \\
\hline $\mathrm{B} 2 \mathrm{O} 3$ & 7.4575 & 7.4075 & & 0.117409 & 0.350011 & 0.178805 & 0.259451 & & 0.219128 \\
\hline $\mathrm{BaO}$ & 2.5325 & 1.76 & & -0.26146 & 0.070988 & -0.17371 & -0.05845 & & -0.11608 \\
\hline $\mathrm{CaO}$ & $3.9 \overline{2}$ & 3.1975 & & -0.07476 & 0.208484 & 0 & 0.098205 & & 0.049103 \\
\hline $\mathrm{Cr} 2 \mathrm{O} 3$ & 0.04625 & 0.0355 & & -0.14215 & 0.158861 & -0.06269 & 0.041669 & & -0.01051 \\
\hline $\mathrm{Cs} 2 \mathrm{O}$ & 0.034 & 0.14 & & 0.787094 & 0.843204 & 0.801904 & 0.821358 & & 0.811631 \\
\hline $\mathrm{Fe} 2 \mathrm{O} 3$ & 0.515 & 0.3325 & & -0.35786 & 0 & -0.2634 & -0.13933 & & -0.20136 \\
\hline $\mathrm{K} 2 \mathrm{O}$ & 1.0725 & 1.4575 & & 0.3549 & 0.524913 & 0.399776 & 0.458721 & & 0.429249 \\
\hline $\mathrm{Li} 2 \mathrm{O}$ & 0.73 & 0.61 & & -0.04913 & 0.22736 & 0.023848 & 0.119711 & & 0.07178 \\
\hline $\mathrm{MgO}$ & 0.0625 & 0.044 & & -0.24528 & 0.08291 & -0.15865 & -0.04486 & & -0.10176 \\
\hline $\mathrm{MnO} 2$ & 0.0085 & 0.00875 & & 0.148374 & 0.372816 & 0.207617 & 0.285433 & & 0.246525 \\
\hline $\mathrm{MoO}$ & 0.155 & 0.1475 & & 0.07875 & 0.32154 & 0.142836 & 0.227014 & & 0.184925 \\
\hline $\mathrm{Na} 2 \mathrm{O}$ & 20.825 & 17.9 & & -0.01993 & 0.248868 & 0.051021 & 0.144216 & & 0.097619 \\
\hline $\mathrm{NiO}$ & 0.004 & 0.01 & & 0.649331 & 0.741748 & 0.673724 & 0.705767 & & 0.689746 \\
\hline $\mathrm{P} 205$ & 0.1525 & 0.2 & & 0.331536 & 0.507706 & 0.378037 & 0.439117 & & 0.408577 \\
\hline $\mathrm{SO3}$ & 0.19 & 0.1052 & & -0.58335 & -0.16606 & -0.4732 & -0.32853 & & -0.40086 \\
\hline $\mathrm{SiO} 2$ & 35.475 & 31.1 & & 0 & 0.263545 & 0.069564 & 0.160938 & & 0.115251 \\
\hline SrO & $0 . \overline{375}$ & 0.098 & & -0.23003 & 0.09414 & -0.14446 & -0.03207 & & -0.08827 \\
\hline $\mathrm{TiO} 2$ & 0.057 & 0.01 & & $-3 . \overline{99704}$ & -2.6801 & -3.64943 & -3.19283 & & -3.42113 \\
\hline $\mathrm{Z̈rO} 2$ & 1.9225 & 1.5175 & & -0.11065 & 0.182059 & -0.03338 & 0.068099 & & 0.017357 \\
\hline $\mathrm{Cl}$ & 0.07 & 0.3606 & & 0.829819 & 0.87467 & 0.841658 & 0.857208 & & 0.849433 \\
\hline$F^{*}$ & 0.03 & 0.301606 & & 0.912799 & 0.935781 & 0.918865 & 0.926833 & & 0.922849 \\
\hline & & 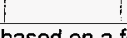 & & 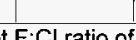 & 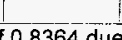 & 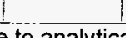 & & & . \\
\hline
\end{tabular}

$$
\text { havend then } h h_{1} a^{\pi} 3 / 2>196
$$




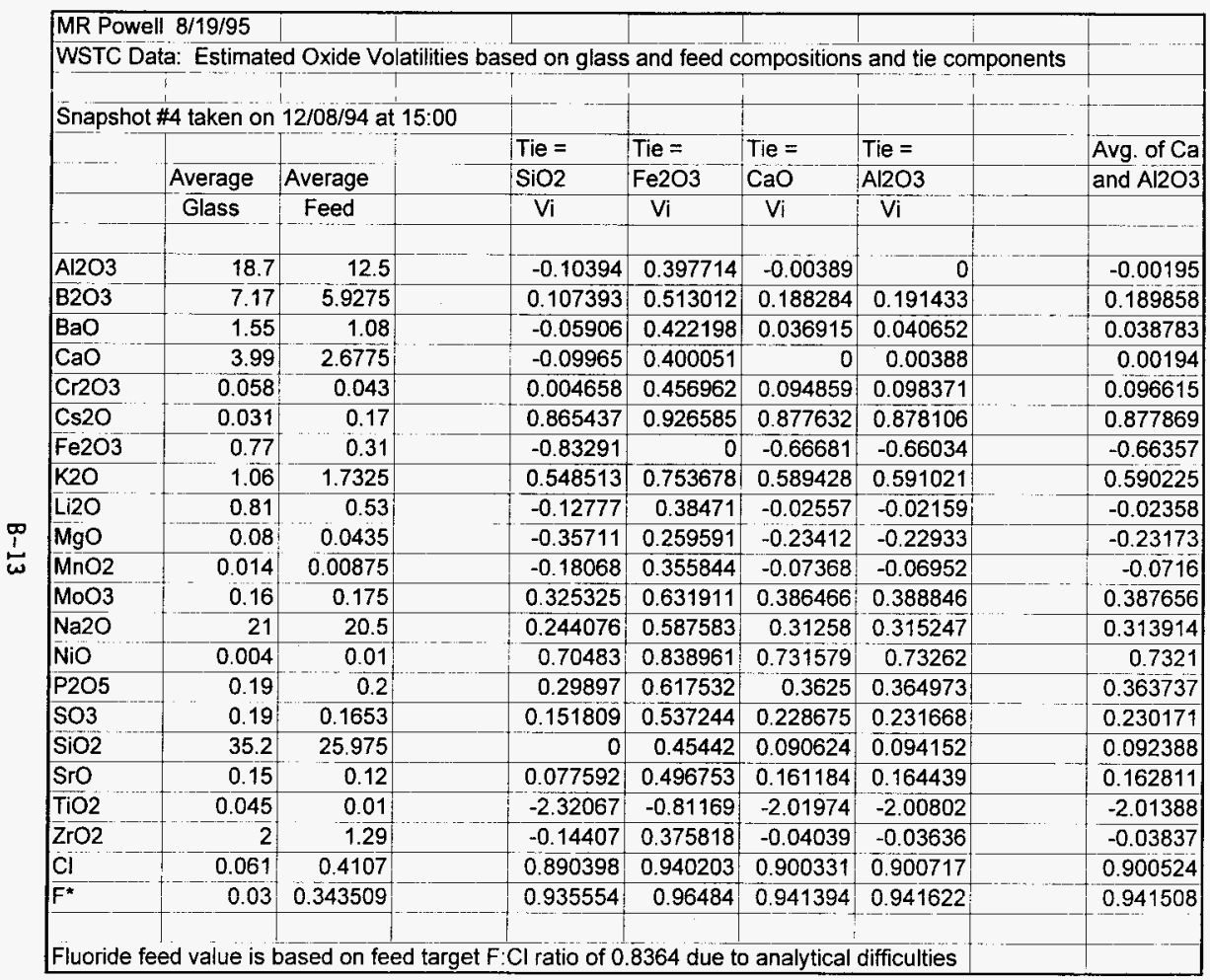

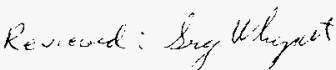

$$
\begin{aligned}
& 3 / 27 / 16
\end{aligned}
$$


WHC-SD-WM-ER-517

Revision 0

This page intentionally left blank. 
WHC-SD-WM-ER-517

Revision 0

APPENDIX C

REVIEW OF UNITED STATES BUREAU OF MINES TEST REPORT, RUN WHC-1 
WHC-SD-WM-ER-517

Revision 0

This page intentionally left blank. 


\section{DON'T SAY IT -- Write It!}

To:

Will Eaton

Subject: $\quad$ Review of USBM test data
Date:

April 28, 1995

From:

I examined the USBM preliminary report along with the recently-obtained sample analytical data. My analyses focused on the data gathered during the 24-hour demonstration test. The following conclusions can be drawn:

\section{Total Entrainment/Volatility:}

The combined entrainment and volatility amounts to $8.7 \pm 2.5 \%$ of the theoretical glass mass. An estimate of $4.0 \pm 2.5 \%$ is obtained if the data are not corrected for the unintended addition of Treehugger feed (estimated to be $540 \mathrm{lbs}$ ). Individual component mass balances are given in Tables $1 \mathrm{a}$ and $1 \mathrm{~b}$. Table 1a has been corrected for the Treehugger feed addition (and gives $8.7 \%$ loss), while Table $1 \mathrm{~b}$ has not (giving $4 \%$ loss). Based on the mass of silica collected in the off-gas system (see Table 3 ), the entrainment amounts to $1.2 \%$ of the theoretical glass mass. The overall mass balance approach (Table 1a) indicates a $1.7 \pm 3.5 \%$ loss of silica. The $1.2 \%$ entrainment estimate is believed to be more accurate (replicate silica analyses for the off-gas solids are more consistent than for the combined feed and glass analyses). However, if off-gas solids accumulations were not fully recovered and weighed, then the $1.2 \%$ entrainment estimate will be too low.

\section{Apparent Addition of Treehugger Feed:}

The glass composition data indicate a net gain of $\mathrm{CaO}$ during the 24 hour test. The mass of $\mathrm{CaO}$ gained by the glass is estimated to be $242 \pm 95 \mathrm{lbs}$ (see Table $1 \mathrm{~b}$ ). It is inferred from the concomitant increase in $\mathrm{TiO}_{2}$ concentration that approximately $540 \mathrm{lbs}$ of "Treehugger" feed became mixed with the WHC glass during the 24 hour test. The Treehugger feed had apparently accumulated on the melter refractory above the glass surface level during an earlier test run. The peak in $\mathrm{CaO}, \mathrm{Al}_{2} \mathrm{O}_{3}$, and $\mathrm{TiO}_{2}$ concentrations observed during the 24 hour run (see plots 1 and 2) can be adequately accounted for by contamination of the WHC glass with Treehugger glass. (see handwritten calculations attached). A significant fraction of the elevation in alumina concentration may have been due to refractory degradation, but this is not certain. The concentrations of $\mathrm{CaO}$ and $\mathrm{TiO}_{2}$ both imply an addition of about $540 \mathrm{lbs}$ of Treehugger feed. If this number is accurate, then $180 \mathrm{lbs}$ of additional alumina would be present in the WHC glass. The feed and glass composition data (Table $1 \mathrm{~b}$ ) show that the additional alumina was $221 \pm 87 \mathrm{lbs}$. Because the predicted $180 \mathrm{lbs}$ alumina is well within the 134-308 $\mathrm{lb}$ range given by this $\pm 87 \mathrm{lb}$ confidence interval, it is not known whether refractory degradation made a significant contribution to the elevated alumina concentrations. It may be possible to determine the refractory contribution (roughly) if we can get $\mathrm{Cr}_{2} \mathrm{O}_{3}$ composition data for both the Treehugger feed and the refractory. 


\section{Calculation of Fractional Volatilities:}

The fractional volatilities of the glass components were estimated based on the ratios of glass and feed oxide concentrations and the assumption of zero $\mathrm{SiO}_{2}$ volatility. These data are presented in Tables $2 a$ and $2 b$. The data in Table $2 a$ have been corrected for the effects of the Treehugger feed on the $\mathrm{SiO}_{2}, \mathrm{CaO}, \mathrm{Al}_{2} \mathrm{O}_{3}$, and $\mathrm{TiO}_{2}$ concentrations (see attached handwritten calculations for correction procedure). The Table $2 \mathrm{~b}$ data have not been corrected. The data in Table $2 \mathrm{a}$ are more accurate. Table $2 \mathrm{~b}$ has been included to show the effect of not correcting for the Treehugger feed addition. Upon applying the correction for the Treehugger feed effects, $\mathrm{Na}_{2} \mathrm{O}$ volatility was estimated to be $20.5 \pm 5.2 \%, \mathrm{~B}_{2} \mathrm{O}_{3}$ volatility was $50.7 \pm 4.4 \%$, and $\mathrm{K}_{2} \mathrm{O}$ volatility was $35.3 \pm 4.5 \%$. Negative volatilities were calculated for $\mathrm{Al}_{2} \mathrm{O}_{3}, \mathrm{CaO}, \mathrm{Cr}_{2} \mathrm{O}_{3}$, $\mathrm{Fe}_{2} \mathrm{O}_{3}, \mathrm{MgO}, \mathrm{TiO}_{2}$, and $\mathrm{ZrO}_{2}$. It is believed that most of these negative volatilities result from the addition of the Treehugger feed to the WHC glass. $\mathrm{Al}_{2} \mathrm{O}_{3}, \mathrm{CaO}, \mathrm{Cr}_{2} \mathrm{O}_{3}, \mathrm{MgO}$, and $\mathrm{TiO}_{2}$ glass concentrations all peak at near 1150 minutes into the 24-hour test (see attached plots 1 and 2). The negative $\mathrm{ZrO}_{2}$ volatility probably results from analytical errors because of the low $\mathrm{ZrO}_{2}$ concentration in the USBM glass. Regardless, all of the computed negative volatilities for these oxides are within their respective $95 \%$ confidence intervals of zero (with the exception of $\mathrm{ZrO}_{2}$ and $\mathrm{MgO}$ ). It may be possible to correct the $\mathrm{ZrO}_{2}$ and $\mathrm{MgO}$ volatilities if we can get a more complete characterization of the Treehugger feed. The $2 \mathrm{wt} \%$ "other" in the given Treehugger composition could easily account for the observed inconsistencies in $\mathrm{ZrO}_{2}$ and $\mathrm{MgO}$ concentrations.

The cause of the negative $\mathrm{Fe}_{2} \mathrm{O}_{3}$ volatility is not known. It is worth noting, however, that the average $\mathrm{Fe}_{2} \mathrm{O}_{3}$ concentration in the feed was within $1 \%$ of the target concentration, but the average $\mathrm{Fe}_{2} \mathrm{O}_{3}$ concentration in the glass was about $12 \%$ higher than the target glass concentration. This implies that there may have been an iron source within the melter. Analytical errors may also be contributing to this discrepancy. The $\mathrm{Fe}_{2} \mathrm{O}_{3}$ concentration for the glass does not show a "peak" at about 1150 minutes (see plot 1), so it is unlikely that this additional ferric oxide can be attributed to the Treehugger feed.

\section{Loss Estimates Based on Collected Off-Gas Solids:}

Using the reported masses of off-gas solids collected during the test and the reported compositions of those solids, \% loss estimates were generated (Table 3). A weighted-average of all the \% loss estimates gives an overall entrainment/volatility loss average of $5.3 \%$ for the 24 hour run. I haven't had time to complete an error analysis on this $5.3 \%$ estimate. I suspect that the confidence interval of the $5.3 \%$ estimate will overlap with the $8.7 \pm 2.5 \%$ estimate given in Table 1a. For now, I recommend using the $8.7 \%$ estimate. It should also be pointed out that the values used for the total Baghouse, Cyclone, and Thermal Oxidizer solids masses are only estimated values. The solids were measured at the end of the test and then it was assumed that since the 24-hour run constituted $59 \%$ of the total melter run time then $59 \%$ of the solids from each of the collection points must have been collected during the 24-hour run. The extent to which this assumption is valid can have a dramatic effect on the $5.3 \%$ loss 
estimate. If all the collected solids were produced solely during the 24-hour run, then the \% loss estimate increases to about $9.0 \%$ (this is an unlikely scenario, though).

The lowest \% loss values calculated in Table 3 were for $\mathrm{SiO}_{2}(1.19 \%)$ and $\mathrm{Fe}_{2} \mathrm{O}_{3}(1.23 \%)$. All others were in excess of $2 \%$. This supports the use of $\mathrm{SiO}_{2}$ as the tie component for the volatility estimates described above. If any of the components had a lower volatility than silica, it would be expected that the \% loss data for those components would be lower than that calculated for silica. Further, the composition of the thermal oxidizer solids (also shown in Table 3) implies that the $\mathrm{SiO}_{2}$ entrainment/volatility was less than that of $\mathrm{Al}_{2} \mathrm{O}_{3}$ and $\mathrm{CaO}$. The thermal oxidizer solids concentrations of alumina and lime are higher (relative to silica and ferric oxide) than would be expected from entrainment alone. It is likely that the elevated level of $\mathrm{CaO}$ is due to entrainment of Treehugger feed or lime. However, it is expected that most of the entichment in $\mathrm{Al}_{2} \mathrm{O}_{3}$ is the result of corrosion of the refractory in the thermal oxidizer which consisted of $80 \mathrm{wt} \% \mathrm{Al}_{2} \mathrm{O}_{3}$.

\section{Review of Feed Pellet Preparation Procedures:}

The Operating Procedure for Feed Preparation was examined in an effort to determine why the prepared feed pellets did not meet target specifications in some cases. Table 4 (attached) shows a comparison of the average feed composition (with $95 \%$ confidence interval in the adjacent column) with the target feed composition. The sodium and potassium concentrations in the feed (and in the resulting glass) were significantly lower than the targets. I reviewed the data given in Tables 4 and 5 of the Operating Procedure and did not find any errors in the procedure used to formulate the simulant recipe. It is possible that the procedure was not followed accurately or maybe there was a calibration problem with their scale. It may be useful to verify the stated glass-former and LLW simulant compositions given in Table 4 of the Operating Procedure. I believe that the specified simulant recipes should have given feed compositions near the targets, so the problem is likely elsewhere. Could something have happened to the sodium and/or potassium in the dryer during denitrification? I can't think of any reasonable possibilities, but that doesn't mean there aren't any. 
Revision 0

\section{Data Sources for USBM Calculations}

This page is intended to aid in the traceability of the data used within this appendix.

Table 2a, 2b Data Sources

Glass average composition and standard deviation are derived from the following glass samples: M1G1-002C, 002U, 004C, 004U, 005C, 005U, 008C, 008U, 010C, 010U, 011U, 011C

Feed average composition and standard deviation are derived from the following feed samples: M1APR-001C, -001U, -002U

M1BPR-022C, -022U, -026U, -043U, -050U

Table 3 data sources

samples analyzed include (average of samples shown)

Baghouse M1B5-001C, -001U

Cyclone M1C4 -001C, $-001 \mathrm{U}$

Thermal Oxidizer M1T3-001C, -001U

Masses Collected in Baghouse, cyclone and thermal oxidizer were initially calculated from the mass of material recovered differ slightly from values given in the report. This is most likely due to differences in the conversion from the sample quantity to the quantity associated with the 24 hour run period. The differences are not deemed significant enough to warrant revision to the calculations.

$\begin{array}{lll} & \text { Values Used } & \text { Page 3-22 of Final Report } \\ \text { Baghouse } & 631 & 645 \\ \text { Cyclone } & 45 & 40 \\ \text { Thermal Oxidizer } & 94 & 97 \\ \text { Feed } & 12954 & 12954\end{array}$

Table 4

Feed Targets are taken from Table 1.1 of test plan. 


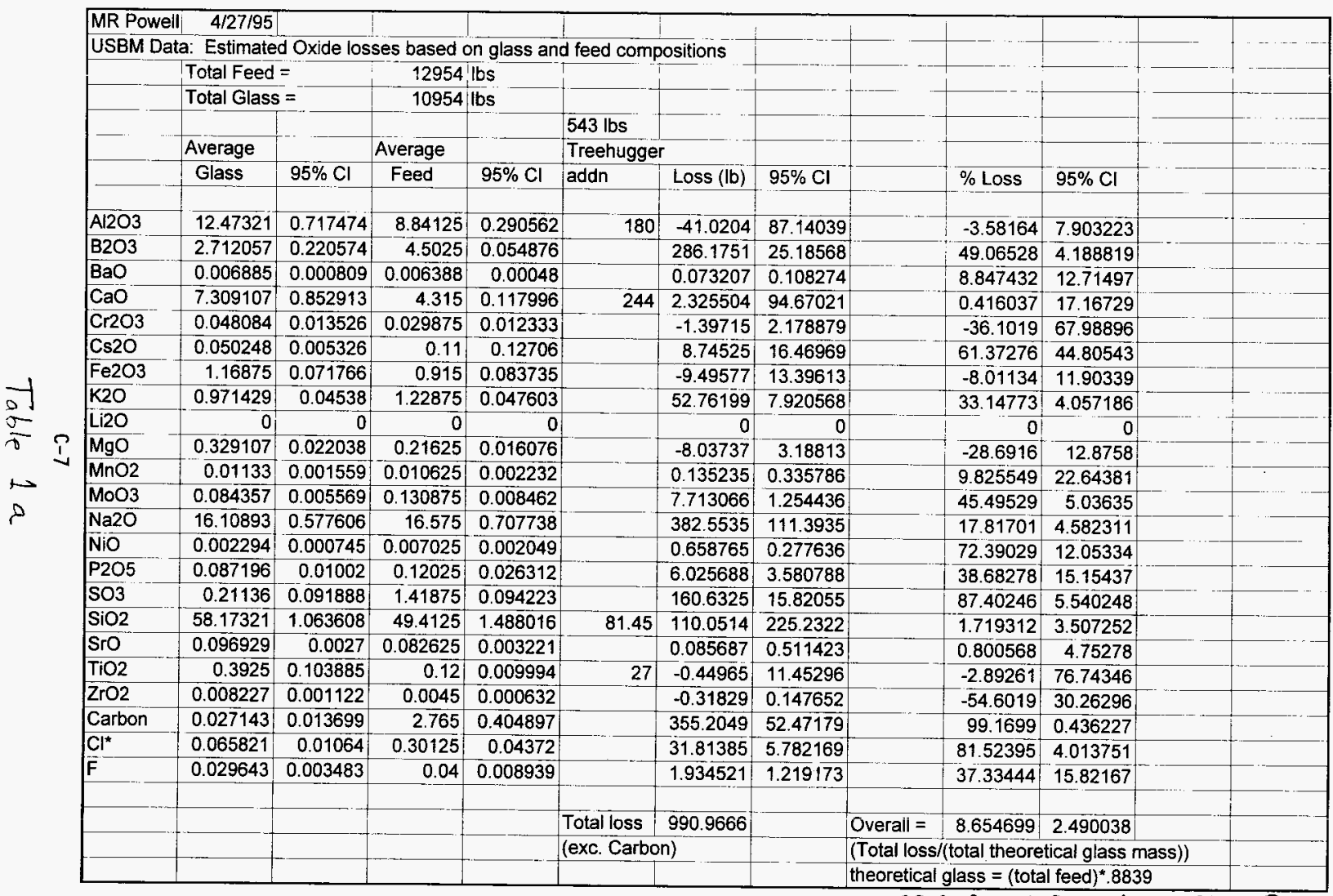

Note: .8839 from LOI sapk MIBPR-022P 


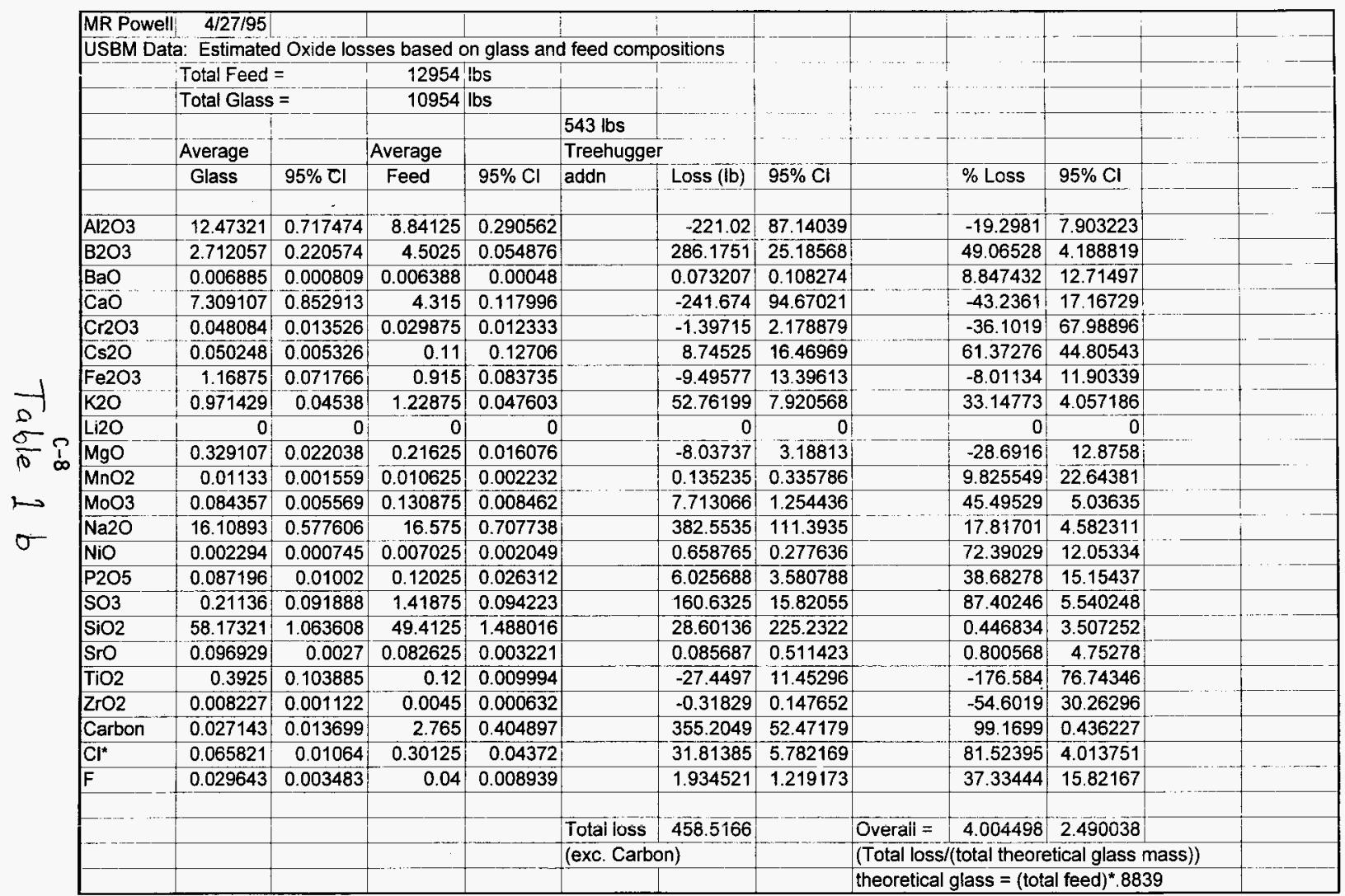




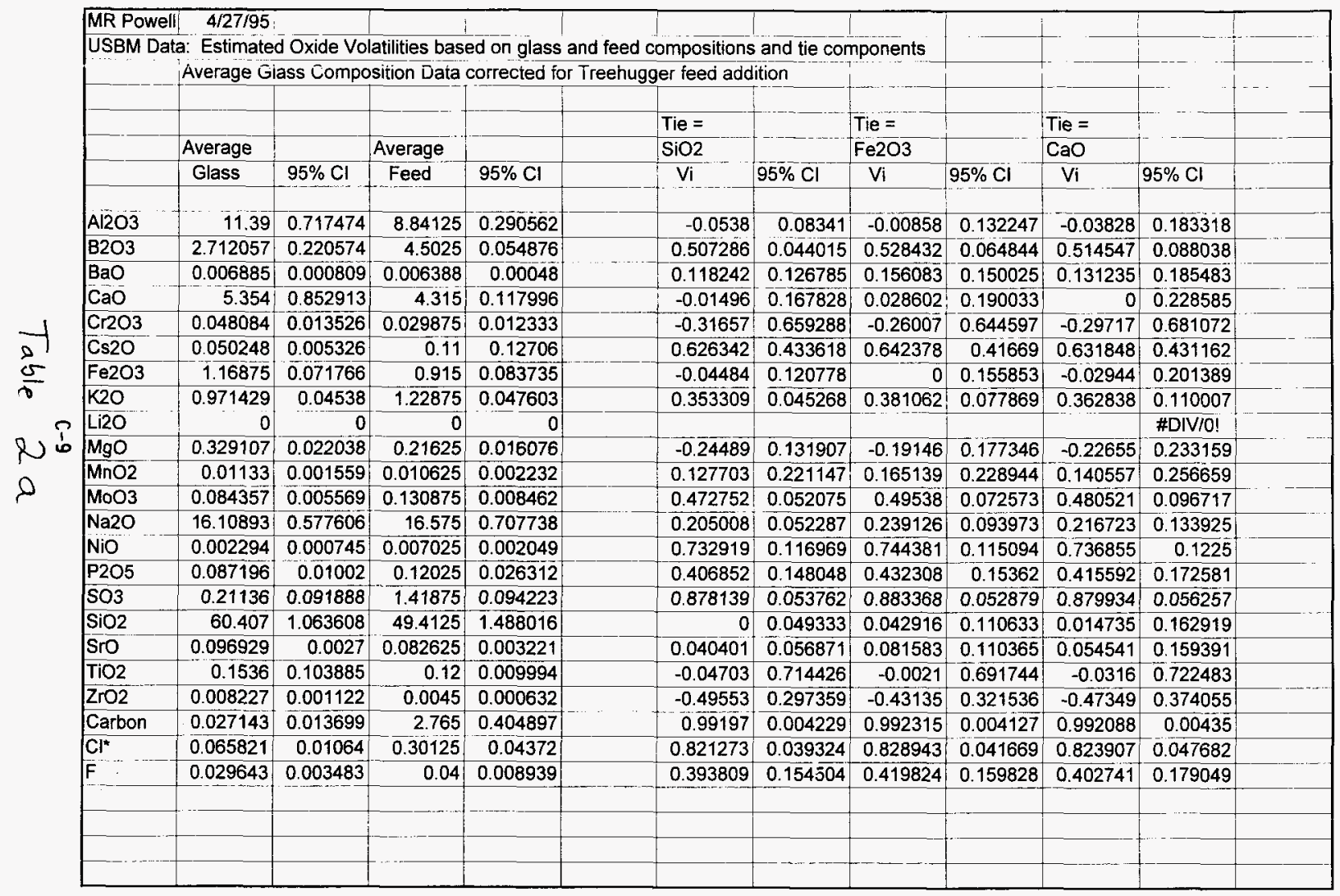




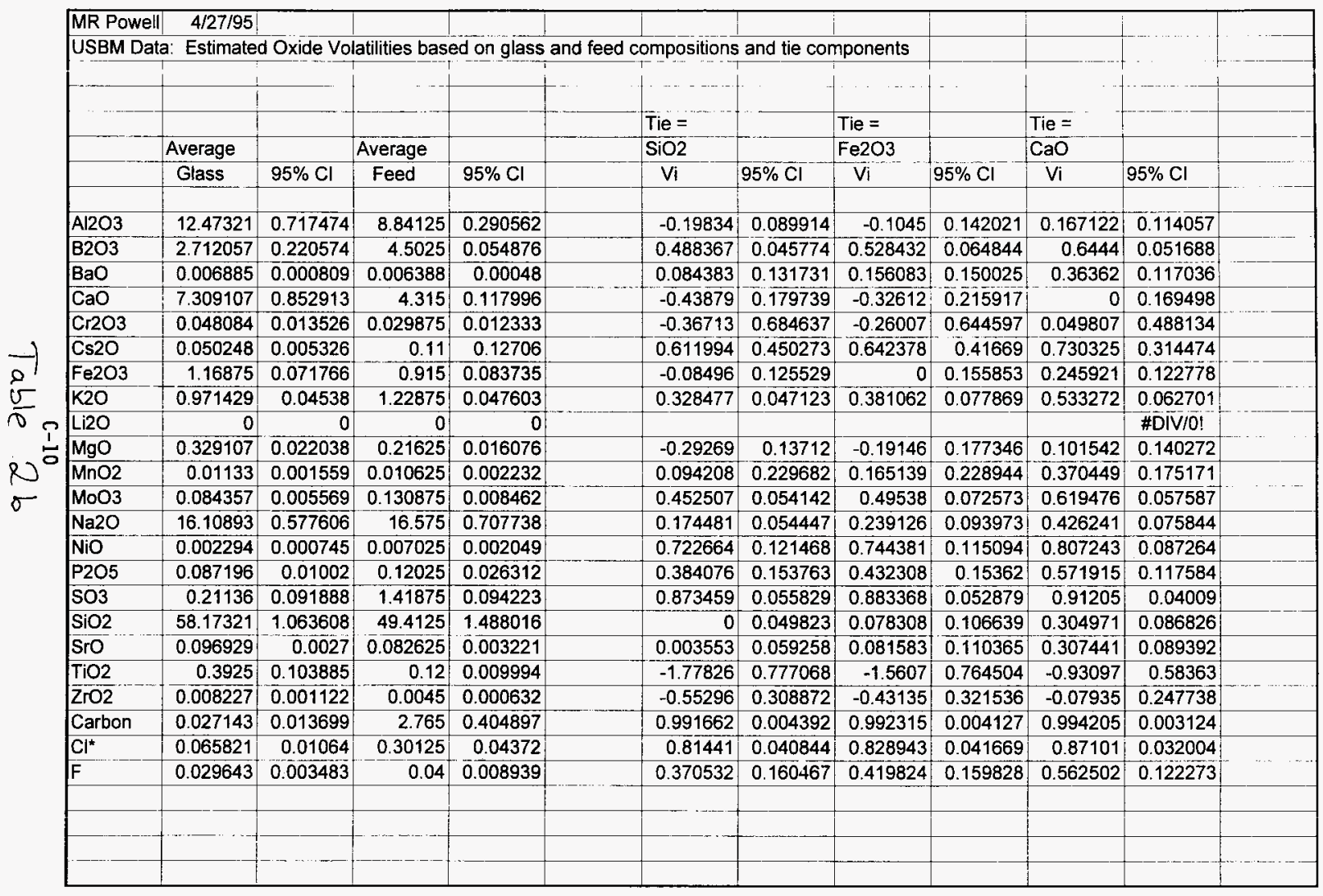

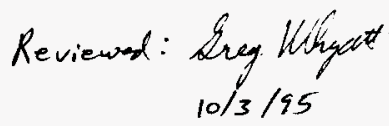




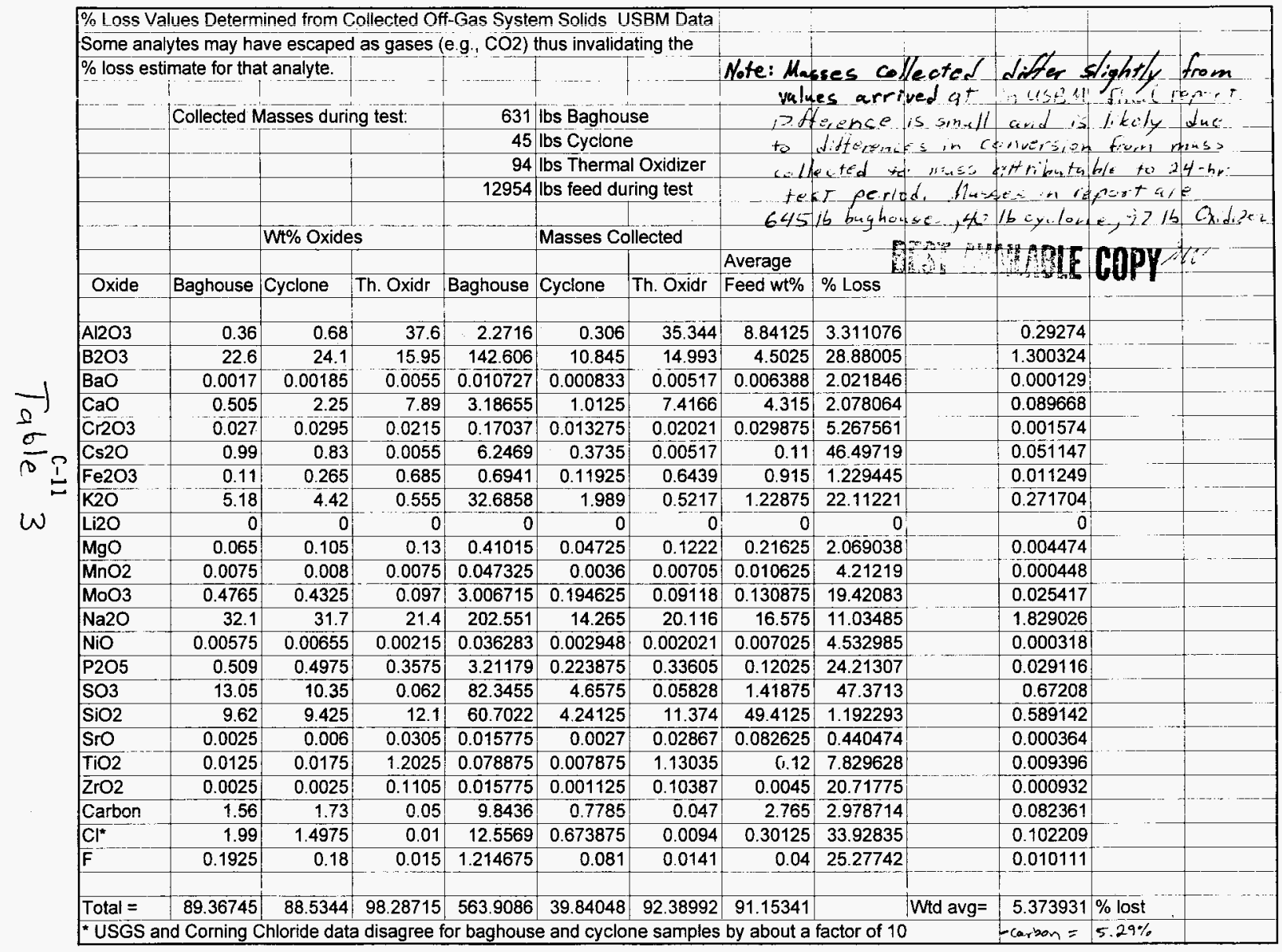




\begin{tabular}{|c|c|c|c|c|c|}
\hline \multicolumn{6}{|c|}{ MR Powell 4/27/95 } \\
\hline & & & & & \\
\hline \multicolumn{6}{|c|}{ Comparison of Target and Actual average Feed Compositions } \\
\hline \multirow{2}{*}{\multicolumn{6}{|c|}{ USBM Data }} \\
\hline & & & & & \\
\hline \multirow{3}{*}{ 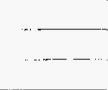 } & & & & & \\
\hline & Average & & Feed & Target & \\
\hline & Feed & $95 \% \mathrm{Cl}$ & Target & Met? & \\
\hline $\mathrm{A} \mid 2 \overline{\mathrm{O}} 3$ & $8 \overline{84125}$ & 0.290562 & 8.968047 & $Y$ & \\
\hline \multirow{2}{*}{$\begin{array}{l}\mathrm{B} 2 \mathrm{O} 3 \\
\mathrm{BaO}\end{array}$} & 4.5025 & 0.054876 & 4.508773 & $\bar{Y}$ & \\
\hline & 0.006388 & 0.00048 & 0 & & \\
\hline $\mathrm{CaO}$ & $4: 315$ & 0.117996 & 4.512308 & $\mathbf{L}$ & \\
\hline $\mathrm{C} 2 \mathrm{O} 3$ & 0.029875 & 0.012333 & 0.035356 & $\bar{Y}$ & \\
\hline \multirow{2}{*}{$\begin{array}{l}\mathrm{Cs} 2 \mathrm{O} \\
\mathrm{Fe} 2 \mathrm{O} 3\end{array}$} & 0.11 & 0.12706 & 0.127282 & $Y$ & \\
\hline & 0.915 & 0.083735 & 0.921907 & $\bar{Y}$ & \\
\hline $\mathrm{K} 2 \mathrm{O}$ & 1.22875 & 0.047603 & 1.447828 & L & \\
\hline $\mathrm{Li} 2 \mathrm{O}$ & 0 & 0 & 0 & & \\
\hline $\mathrm{MgO}$ & 0.21625 & 0.016076 & 0.279312 & $\mathbf{L}$ & \\
\hline \multirow{2}{*}{$\mathrm{MnO2}$} & 0.010625 & 0.002232 & 0.001768 & $\mathrm{H}$ & \\
\hline & 0.130875 & 0.008462 & 0.129049 & $Y$ & \\
\hline $\mathrm{Na} 2 \mathrm{O}$ & 16.575 & 0.707738 & 18.06603 & L & \\
\hline $\mathrm{NiO}$ & 0.007025 & 0.002049 & 0 & & \\
\hline $\mathrm{P} 205$ & 0.12025 & 0.026312 & 0.188271 & $L$ & \\
\hline \multirow{2}{*}{$\frac{\mathrm{SO} 3}{\mathrm{SiO} 2}$} & 1.41875 & 0.094223 & 0.219207 & $\mathrm{H}$ & \\
\hline & 49.4125 & 1.488016 & 47.33018 & $\mathrm{H}$ & \\
\hline $\begin{array}{l}\mathrm{SiO} 2 \\
\mathrm{SiO}\end{array}$ & 0.082625 & 0.003221 & 0.091926 & $L$ & \\
\hline $\mathrm{TiO} 2$ & 0.12 & 0.009994 & 0 & & \\
\hline $\mathrm{ZrO} 2$ & 0.0045 & 0.000632 & 0 & & \\
\hline Carbon & 2.765 & 0.404897 & 0 & & \\
\hline \multirow{2}{*}{$\begin{array}{l}\mathrm{Cl} \\
\mathrm{F}\end{array}$} & 0.30125 & 0.04372 & 0.304062 & $\bar{Y}$ & \\
\hline & 0.04 & 0.008939 & 0.254563 & $L$ & \\
\hline \multicolumn{6}{|c|}{ Feed target estimated using target glass composition data } \\
\hline \multirow{2}{*}{\multicolumn{6}{|c|}{$\begin{array}{l}\text { Feed target estimated using target glass composition data } \\
\text { given in Table } 1.1 \text { of test plan. Target glass concentrations } \\
\text { were multiplied by } 0.8839 \text { to account for mass loss upon }\end{array}$}} \\
\hline & \multicolumn{5}{|c|}{ were multiplied by 0.8839 to account for mass loss upon } \\
\hline melting. & & & & & \\
\hline
\end{tabular}

Note: faed targets from Talle 1.1 are for A pellets while .8839 is LoI for B pollets. Discrepancy is small howeven. SW

Table 4 
WHC-SD-WM-ER-517

Revision 0

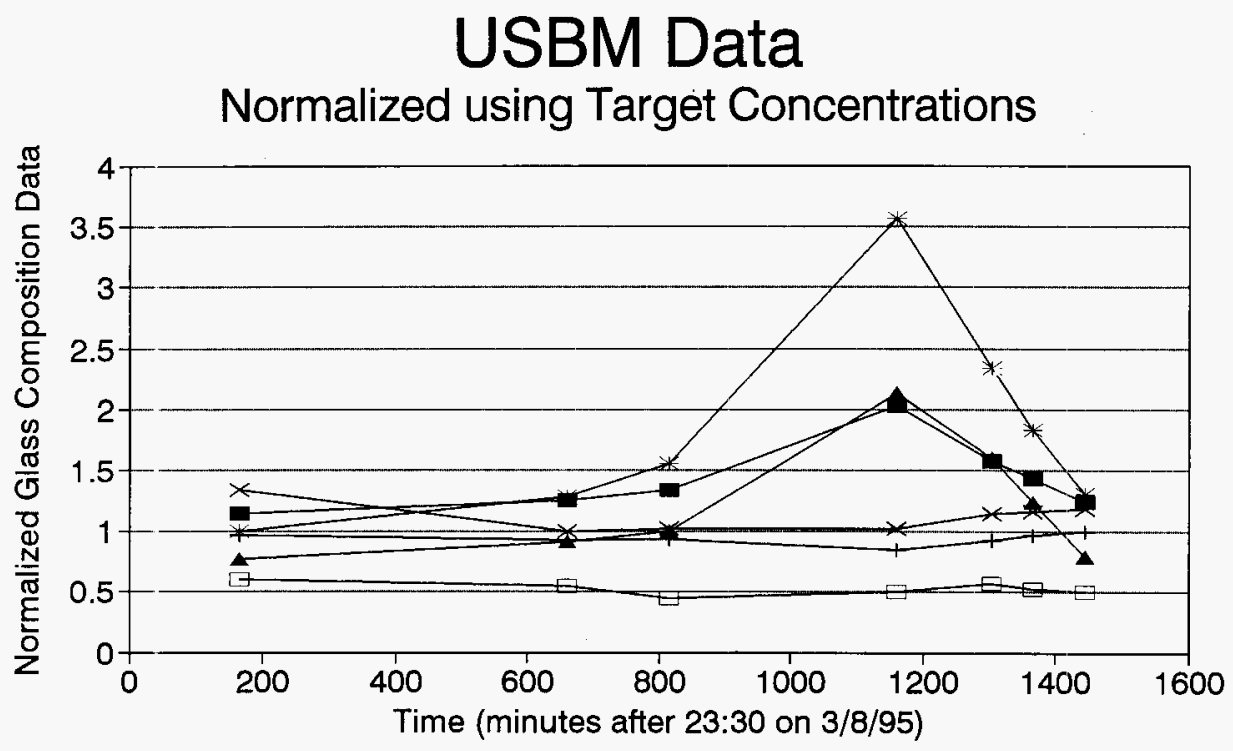

PLOT 1

c-13 


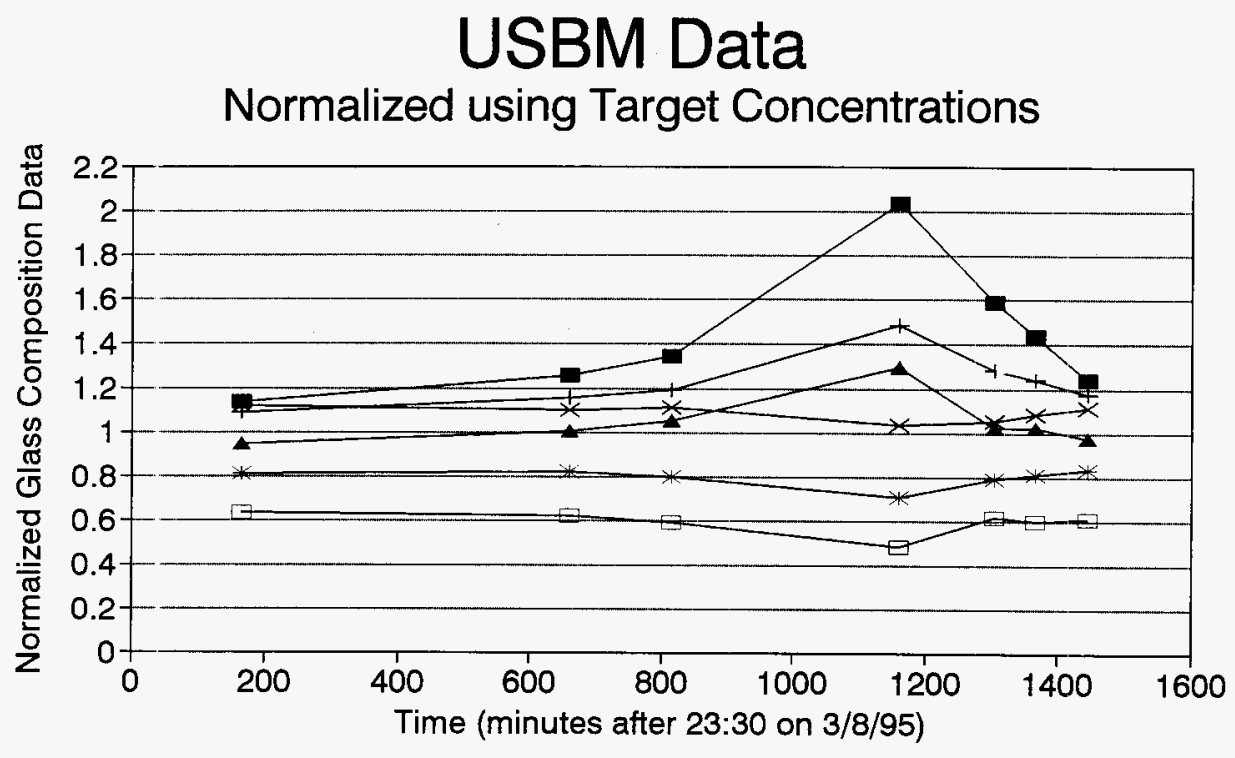

$$
\begin{aligned}
& \rightarrow \mathrm{CaO} \leftarrow \mathrm{Al} 2 \mathrm{O} 3 \rightarrow \mathrm{Na} 2 \mathrm{O} \\
& -\mathrm{K} 2 \mathrm{O} \rightarrow \mathrm{SiO} 2 \leftarrow \mathrm{MgO}
\end{aligned}
$$


Revision 0

Batlelle

\begin{tabular}{|l|l|}
\hline \multirow{2}{*}{ CALCULATION WORKSHEET } & Cake. No. \\
\cline { 2 - 2 } & Page \\
\hline
\end{tabular}

During $\mathrm{CaO}$ spike,

$\mathrm{TiO}_{2}$ increased from target $\equiv .214 \mathrm{wi} \%$ to $.763 \mathrm{w}+\%$

$\mathrm{CaO}$ increased fan target $=5.105 \mathrm{wt} \%$ to $10.35 \mathrm{ut \%}$

$\mathrm{Al}_{2} \mathrm{O}_{3}$ increased from target $=10.146 \mathrm{wtw}$ to $1505 \mathrm{w} 1 \%$

$\mathrm{SiO}_{2}$ decreased from initial $\approx 59.7 \mathrm{w} \% \pm 2$ to $553 \mathrm{w} \%$

Trechugger composition was approximately

$$
\begin{array}{ll}
15 \% & 6 . \mathrm{O}_{2} \\
45 \% & \mathrm{C} O \\
33 \% & \mathrm{Al}_{2} \mathrm{O}_{3} \\
\frac{5 \%}{98 \%} & \mathrm{TiO}_{2}
\end{array}
$$

Let $x$ = mass of treekugger added to glass to get the "peak" concentrations. using 60 as basis gives:

$$
\frac{5.105+.45 x}{100+x}=.1035 \Rightarrow x=15.14 \frac{g \text { true huger }}{100 \text { w wii glass }}
$$

Using $x=15.14$ to estimate expected $\mathrm{T}_{1} \mathrm{O}_{2}$ concentration,

$$
w t \% \mathrm{TiO}_{2}=\frac{.214+(.05)(15.14)}{115.14} \times 100 \%=0.84 \mathrm{wt \%}
$$

Using $T: O_{2}$ as basis gives:

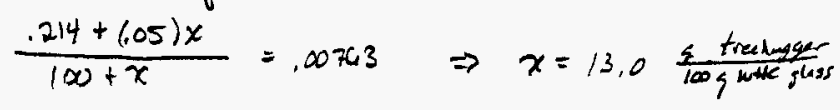

Using $x=13.0$ to estimate COO cone gins:

$$
\omega t \% \mathrm{CaO}=\frac{5.105+(.45)(13)}{113} \times 100 \%=9.7 \mathrm{ut} \%
$$

BEST MURE COPY

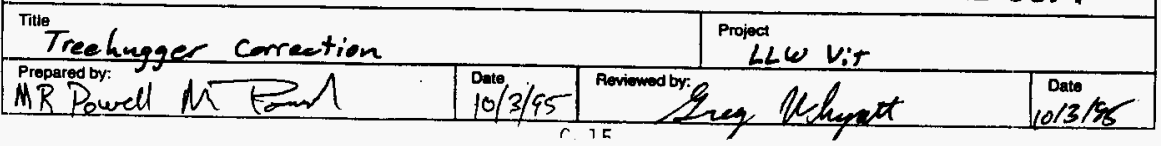


Revisioilo

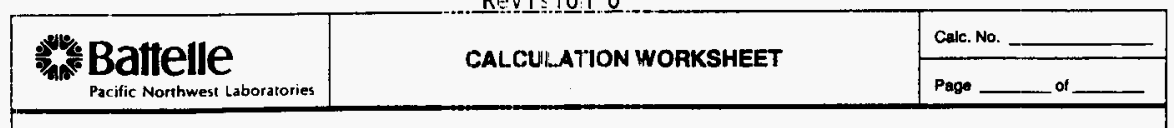

Assuming $\mathrm{THO}_{2}$ estimate for $\chi$ is best, should be 0.66 to estimate mass of any additional $\mathrm{CaO}$.

First estimate $\left[\mathrm{Al}_{2} \mathrm{O}_{3}\right]$ and $\left[\mathrm{S}_{1} \mathrm{O}_{2}\right]$ using $x=13,0$

$$
\begin{aligned}
& {\left[A l_{2} O_{3}\right]=\frac{10.146+(13)(.33)}{113} \times 100 \%=12.0 \mathrm{w1 \%}} \\
& {[5.0 .0]=\frac{59.7+(13)(.15)}{113} \times 100 \%=54.6 \mathrm{w0} \%}
\end{aligned}
$$

$\mathrm{SiO}_{2}$ estimate is within exp error of mustard. wi\% $5 . \mathrm{O}_{2}$. $\mathrm{Al}_{2} \mathrm{O}_{3}$ estimate is significantly low. Therefore expect that some additional $\mathrm{Al}_{2} \mathrm{OS}$ was ad ed to the glass as a result of refractory wear.

Also a fraction of the added C.O (Prob 10-20\%) may have come from original GaO addition -. tough to jade there things though - errors in analytical data candor treehuger fammlation can be significant.

Overall conclusion: Most of the Cal spite 3 due to trehugger material.

How much Treehugger?

Based on Table Ia,

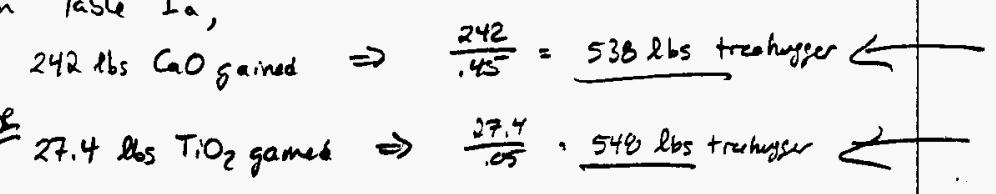

This wail preset that Altos gamed $=(543)(.33)=179$ lbs

This is within the CI of the lbs of $\mathrm{Al}_{2} \mathrm{O}_{3}$ gained $\Rightarrow 2215$ eq $\mathrm{lbs}_{\mathrm{A}} \mathrm{H}_{2} \mathrm{O}_{3}$ gand.

BES MPMUERE COPY

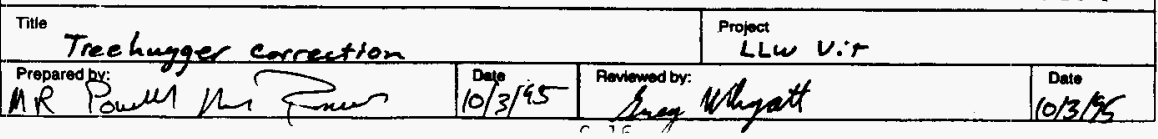


WHC-SD-WM-ER-517

Revision 0

This page intentionally left blank. 
WHC-SD-WM-ER-517

Revision 0

APPENDIX D

REVIEW OF UNITED STATES BUREAU OF MINES TEST REPORT, RUNS WHC-2, 3

D-1 
WHC-SD-WM-ER-517

Revision 0

This page intentionally left blank.

D-2 
DON'T SAY IT - - Write It!

To: Will Eaton
Date: September 5, 1995

From: Greg Whyatt

Subject: Review of WHC2 and WHC3 Run Report Data

I have reviewed the sections of the USBM final report (EDT 611877) dealing with the additional work performed by the USBM after initial phase I testing was completed. The tests are designated as WHC2 and WHC3.

\section{WHC2}

The primary change made prior to $\mathrm{WHC} 2$ was a reduction in the voltage and power input to the furnace. The furnace was initially full of glass and was heated from 07:35 to 11:35 after which type B feed was added and at 12:30 the furnace was tapped. The offgas exit port was plugged or impaired for much of the operation time and the test was terminated at 17:24.

The report cites increasing concentrations of sodium and boron in the product glass as evidence of significant improvements (reductions in volatility) obtained due to the lower power operation. This claim appears to be substantiated. My estimate of the volatility (discussed below) is $20-25 \%$ loss of boron and $16-23 \%$ loss of sodium for the WHC2 test, based on an assumption that the process was at steady state when the final glass sample was taken. Mike Powell had earlier estimated that the losses during the WHC1 test were $50.7+/-4.4 \%$ for Boron and $20.5+/-$ $5.2 \%$ for sodium.

The report claims that the vaporization losses were reduced to $0.57 \%$. Based on analysis of sample WHC2-SP3, I conclude that this claim is overly optimistic. Based only on sodium and boron loss estimates the loss in the glass mass is about $5.0 \mathrm{wt} \%$. A statement is made that the off gases accounted for $11.1 \%$ of the furnace feed but no data is provided to support this. The mass balance provided does not explain how the mass of glass initially in the melter and left in the melter at the end of the run is determined. Thus, I am unable to support conclusions about losses 
of volatile or semi-volatile materials based on the mass balance.

\section{Offgas Calculations for WHC2}

The glass compositions still contain evidence of the contamination by Tree-Hugger feed introduced to the furnace to start the WHC1 test. The final glass sample (WHC2-SP3P(2)) was selected as the most useful sample information to characterize the operating conditions because:

1) Initial heating and prior operation at higher temperatures in WHCl likely resulted in lower concentrations of volatile components in the glass at earlier times. Thus, the earlier glass samples are believed to be less representative of the eventual steady state condition.

2) The analytical information is more complete than for other samples.

Based on increases in the $\mathrm{TiO}_{2}, \mathrm{CaO}$ and $\mathrm{Al} \mathrm{Q}$ content over the target values, the glass is apparently still being contaminated with Treehugger feed material. Using the $\mathrm{TiO}_{2}$ content, the glass composition was adjusted to remove the influence of the Treehugger contamination. This results in tie -component results that are more consistent. $\mathrm{FeO}, \mathrm{SiO}_{2}, \mathrm{CaO}$, and $\mathrm{Al}_{2} \mathrm{O}_{3}$ were used as tie components to estimate volatility. However, the most reliable results are believed to be obtained from the $\mathrm{FeO}$ and $\mathrm{Al}_{2} \mathrm{O}_{3}$ due to the difficulty in getting good analytical data on $\mathrm{SiO}_{2}$ and the potential errors in $\mathrm{CaO}$ content resulting from the use of $\mathrm{CaO}$ as a fluxing agent and the fact that it is present as $\mathbf{4 5} \mathrm{wt} \%$ of the Treehugger feed. Calculations were performed using FeO and $\mathrm{Al}_{2} \mathrm{O}_{3}$ as tie components and for both a feed composition matching the analytical results from WHC1 method B feed and also based on the feed target values from the testplan. This results in four different volatile loss values. The range of values obtained is provided in Table 1 . Note that the ranges provide the range of values obtained for different tie component and feed composition assumptions and do not represent a confidence interval. All results are based on only a single glass analysis result. 
WHC-SD-WM-ER-517

Revision 0

Table 1. Volatility loss estimates for WHC2 sample WHC2-SP3

\begin{tabular}{|l|l|l|}
\hline Oxide Component & Minimum Percent Loss ${ }^{a}$ & Maximum Percent Loss ${ }^{a}$ \\
\hline $\mathrm{Al}_{2} \mathrm{O}_{3}$ & -6 & 0 \\
\hline $\mathrm{B}_{2} \mathrm{O}_{3}$ & 20 & 25 \\
\hline $\mathrm{BaO}$ & -219 & -209 \\
\hline $\mathrm{CaO}$ & -8.8 & -2.5 \\
\hline $\mathrm{Cr}_{2} \mathrm{O}_{3}$ & -91 & -68 \\
\hline $\mathrm{FeO}$ & 0 & 5.8 \\
\hline $\mathrm{K}_{2} \mathrm{O}$ & -156 & -130 \\
\hline $\mathrm{MgO}$ & -42 & -50 \\
\hline $\mathrm{MnO}_{2}$ & -856 & -517 \\
\hline $\mathrm{MoO}_{3}$ & 47 & 53 \\
\hline $\mathrm{Na}_{2} \mathrm{O}^{\circ}$ & 16 & 23 \\
\hline $\mathrm{P}_{2} \mathrm{O}_{5}$ & -50 & -34 \\
\hline $\mathrm{SO}_{3}$ & -318 & -288 \\
\hline $\mathrm{SiO}_{2}$ & 10 & 16 \\
\hline $\mathrm{SrO}$ & -10 & 1.6 \\
\hline $\mathrm{TiO}_{2}{ }^{\circ}$ & 4.3 & 7.3 \\
\hline $\mathrm{ZrO}_{2}{ }^{\circ}$ & -91 & -85 \\
\hline
\end{tabular}

Negative Values indicate a gain (i.e. presence in excess of expected based on feed analysis composition and test plan target values.)

b These components do not have test plan target compositions so the range of values is based only on the feed analysis values. 
Volatility of boron was reduced substantially from the WHC1 test. The effect on sodium volatility was less obvious. A number of the components in Table 1 show negative values which indicate increases above the amount present in the feed. Some such as $\mathrm{Cr}_{2} \mathrm{O}_{3}$ may be due to refractory corrosion and contamination from the Treehugger feed. Others such as $\mathrm{Ba}, \mathrm{Mn}$, and $\mathrm{ZrO}_{2}$ are present is such small quantities in the glass that a small amount of contamination relates to a large percentage change. However, $\mathrm{MgO}$ and $\mathrm{SO}_{3}$ are roughly $0.25 \%$ of oxides in the feed and show significant increases from unknown sources. The most unusual component was the $\mathrm{K}_{2} \mathrm{O}$ which has a feed target of $1.71 \mathrm{wt} \%$ and increased to $4.25 \mathrm{wt} \%$ in the glass. No reason for the elevated $\mathrm{K}_{2} \mathrm{O}$ levels is known. 


\section{WHC3}

During the WHC3 test, the electrodes were made larger to reduce the energy density of the electrodes to $1 / 4$ of the previous configuration. A lance for injection of air or oxygen was installed and failed prior to introduction of method B feed. Graphite inserts intended to control the flow in the drain were tested but failed to provide continuous tapping. The furnace was started on "about $1000 \mathrm{lb}$ " of crushed INEL glass and $98 \mathrm{lb}$ of carbon steel punchings. An additional $200 \mathrm{lb}$ carbon steel punchings were later added to the melter. After 13 hours, the bottom drain was opened and the furnace was emptied. The remainder of the glass and metal in the melter was sufficient to plug the drain hole. Method B pellets were then added and the melter operated. As has been experienced in previous tests, the drain froze and an addition of $303 \mathrm{lb}$ of $\mathrm{CaO}$ was needed fluidize the melt. Four glass samples were taken as well as a cyclone and baghouse sample.

\section{ANALYSIS OF DATA}

Determination of split factors through comparison of the feed and glass composition is complicated by the fact that potential tie components may have significant contamination present due to addition of $\mathrm{CaO}$, iron punchings, possible INEL glass residue of unknown composition, and remaining residue from the Treehugger feed utilized in test WHC1. The analytical results from the four glass samples were adjusted to remove the excess iron and calcium content based on the $\mathrm{SiO}_{2}$ and $\mathrm{Al}_{2} \mathrm{O}_{3}$ content. The glass composition was then normalized to estimate the glass composition without the extra calcium and iron. The results obtained using $\mathrm{SiO}_{2}$ compare very well with the results obtained using $\mathrm{Al}_{2} \mathrm{O}_{3}$ and the two results were averaged to arrive at the glass composition without the excess $\mathrm{CaO}$ and $\mathrm{FeO}$. No adjustments were made to the $\mathrm{TiO}_{2}$ content although it is noted that it is high by about a factor of 2 which is likely due to contamination by the Treehugger feed material which was $5 \mathrm{wt} \% \mathrm{TiO}_{2}$. After adjustment of $\mathrm{CaO}$ and $\mathrm{FeO}$ and normalization, the compositions for the four samples were evaluated using $\mathrm{SiO}_{2}$ and $\mathrm{Al}_{2} \mathrm{O}_{3}$ as tie components and the analysis of feed data from Table 3.5 of the WHC1 report. The volatility 
estimates for each component obtained using each of the two tie components were in good agreement and were averaged for each component. The results are provided in Table 1 . The results are also plotted in the attached figure, which provides a convenient visualization of the volatility results.

Table 1. Estimated Loss of Oxide Components. Data is provided for each of four glass samples. Negative values indicate a gain in composition above the feed analysis.

\begin{tabular}{|l|r|r|r|r|r|}
\hline Oxide & \multicolumn{3}{|c|}{ Mass Fraction Loss of Oxide Component } & Average Percent \\
\hline $\mathrm{Component}$ & $19: 55$ & $20: 42$ & $21: 19$ & $00: 42$ & Loss \\
\hline $\mathrm{Al}_{2} \mathrm{O}_{3}$ & 0 & 0.0175 & 0.001 & 0.001 & 0.49 \\
\hline $\mathrm{B}_{2} \mathrm{O}_{3}$ & 0.19268 & 0.14748 & 0.21917 & 0.14804 & 17.68 \\
\hline $\mathrm{BaO}$ & -2.3538 & -2.3499 & -2.4959 & -2.5392 & -243.47 \\
\hline $\mathrm{Cl}$ & 0.96558 & 0.97355 & 0.9862 & 0.97206 & 97.44 \\
\hline $\mathrm{CaO}$ & -0.016 & -0.016 & -0.016 & -0.016 & -1.61 \\
\hline $\mathrm{Cr}_{2} \mathrm{O}_{3}$ & -0.8294 & -0.3704 & -0.6685 & -0.6892 & -63.94 \\
\hline $\mathrm{Cs}_{2} \mathrm{O}$ & 0.36555 & 0.3855 & 0.45223 & 0.344 & 38.68 \\
\hline $\mathrm{F}$ & 0.9968 & 0.9968 & 0.99332 & 0.99324 & 99.5 \\
\hline $\mathrm{FeO}$ & -0.016 & -0.016 & -0.016 & -0.016 & -1.57 \\
\hline $\mathrm{K}_{2} \mathrm{O}$ & 0.26452 & 0.25362 & 0.27015 & 0.22385 & 25.3 \\
\hline $\mathrm{MgO}$ & -0.6903 & -0.5678 & -0.9717 & -0.7413 & -74.28 \\
\hline $\mathrm{MnO}_{2}$ & -5.7076 & -5.6998 & -7.3902 & -5.7245 & -613.06 \\
\hline $\mathrm{MoO}_{3}$ & 0.51275 & 0.507 & 0.4987 & 0.29215 & 45.27 \\
\hline $\mathrm{Na}_{2} \mathrm{O}$ & 0.0817 & 0.0555 & 0.16318 & -0.041 & 6.48 \\
\hline $\mathrm{P}_{2} \mathrm{O}_{5}$ & 0.45368 & 0.54526 & 0.57289 & 0.5676 & 53.49 \\
\hline $\mathrm{SO}_{3}$ & 0.9165 & 0.9166 & 0.95648 & 0.95594 & 93.64 \\
\hline $\mathrm{SiO}_{2}$ & -0.9913 & -0.989 & -1.2942 & -0.8802 & -0.51 \\
\hline $\mathrm{TiO}_{2}$ & -7.0491 & -5.0298 & -7.3902 & -5.3706 & -103.87 \\
\hline $\mathrm{ZrO}_{2}$ & & -0.018 & & & -621 \\
\hline & & & 0 & \\
\hline
\end{tabular}


The glass analysis indicates quantities of $\mathrm{BaO}, \mathrm{Cr}_{2} \mathrm{O}_{3}, \mathrm{MgO}, \mathrm{MnO}_{2}, \mathrm{TiO}_{2}$ that range from $64 \%$ to $613 \%$ in excess of that in the feed. These components likely come from steel punchings, refractory corrosion, Treehugger feed and possibly INEL glass. However, the precise sources are impossible to determine. Comparison of volatility losses to the results of the WHC1 test creates some confusing observations. The volatility of $\mathrm{B}_{2} \mathrm{O}_{3}, \mathrm{Na}_{2} \mathrm{O}, \mathrm{Cs}_{2} \mathrm{O}$ and $\mathrm{K}_{2} \mathrm{O}$ was reduced significantly. The volatility of the major oxides $\mathrm{Na}_{2} \mathrm{O}$ and $\mathrm{B}_{2} \mathrm{O}_{3}$ was reduced to about $1 / 3$ of the volatility observed in WHC1. The volatility of $\mathrm{Cs}_{2} \mathrm{O}$ was roughly $64 \%$ of the previous quantity and $\mathrm{K} . \mathrm{O}$ volatility was $77 \%$ of its previous rate. However, the estimates for volatility of $\mathrm{Cl}, \mathrm{F}$, $\mathrm{SO}_{3}$ and $\mathrm{P}_{2} \mathrm{O}_{5}$ were much worse for $\mathrm{WHC} 3$ than in $\mathrm{WHC1}$. No reason is known why the volatility of these components would be worse under the WHC3 operating conditions. Volatility of $\mathrm{MoO}_{3}$ was roughly unchanged.

The USBM report makes several claims for the WHC3 run, among them:

\section{1) Sodium present in glass in excess of feed}

While my calculations do indicate that the final sample contained a $4 \%$ excess of sodium, I suspect that this is an analytical problem as sodium clearly deviates from other analytes which can be seen in the attached figure. I would conclude that the average of the four samples which is $6.5 \%$ loss is a better value for sodium volatility.

2) Silica present in excess of feed.

My silica values average to $0.5 \mathrm{wt} \%$ gain in silica which is essentially no volatility which is what would be expected.

3) Over $88 \%$ Boria reports to Glass.

I get a slightly less favorable result for Boria of $18 \%$ loss average and $15 \%$ loss if based on the last sample only. This is only a small discrepancy.

4) Over $77 \%$ Cesia reports to Glass.

I calculate a $39 \%$ loss of cesium which is somewhat greater than the $<23 \%$ loss provided by USBM. 
5) Over $1 / 2$ of Chloride was lost.

I have a major discrepancy on this item as I calculate $97 \%$ of the chloride as being lost from the glass. How USBM reached the lower value is not known.

6) Over $90 \%$ of Fluoride was lost.

I calculate $99.5 \%$ of the fluoride lost which may be somewhat different than greater than 90\% loss. Analytical difficulties have been experienced in the past on fluoride so the accuracy of this value is suspect in the absence of additional information.

The material collected from the offgas system was not used to estimate the volatile losses because the collected material in most cases represents only a small fraction of the material that was not present in the glass. For example the mass balance for $\mathrm{B}_{2} \mathrm{O}_{3}$, indicates that only $18 \%$ of the material not reporting to the glass was recovered in the cyclone or the baghouse. The discrepancy is worse for species which may escape as a gas such as $\mathrm{SO}_{3}$. 
WHC-SD-WM-ER-517

Revision 0

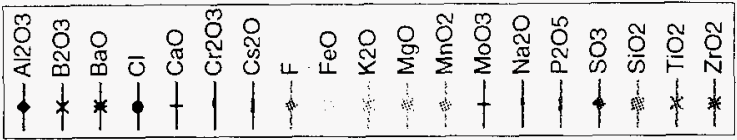

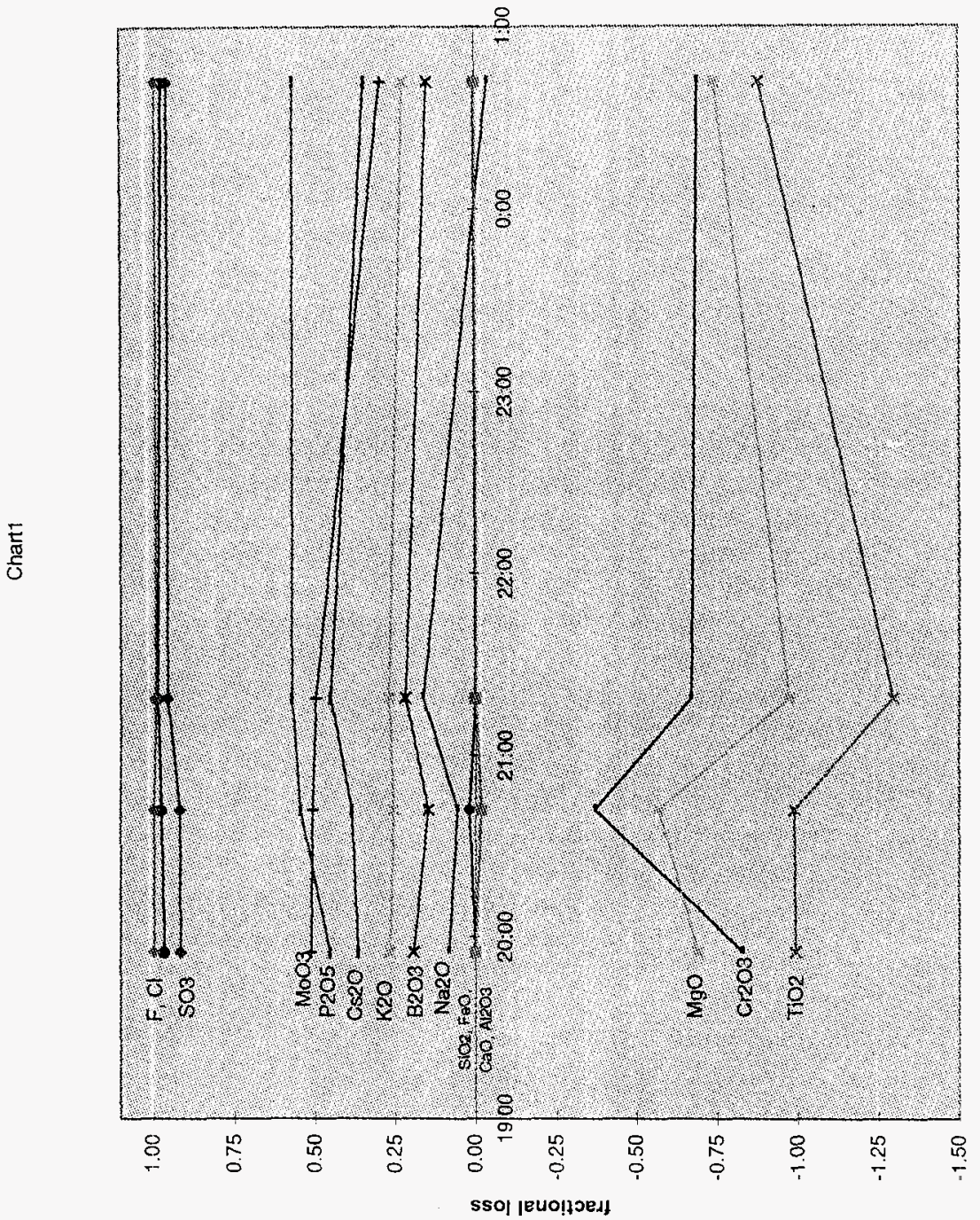

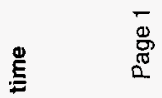




$$
H^{\prime \prime}-(-5)-n^{\prime} M-E F-517
$$

Revision 0

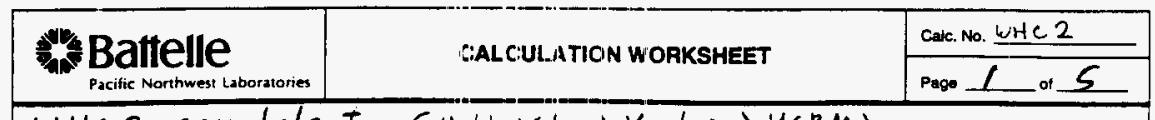

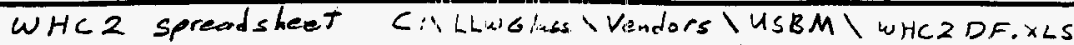

Col A - labels for oxides

col B - Target Composition bused o. Table 3.5 of report for WHCI

col C - Column intentionally blank

ColD - Target Composition bused on feed composition target in test plan Table 13 Jan 12, 55 Test plan

col E - Glass Composition of Simple wHC2-5P3P(2) fro..

$$
\text { Table } 4.2 \text { of report }(2 / 31 / 55)
$$

Col - Loss estimate based ar $5, \mathrm{O}_{2}$ tic Calculation and $B$ feed Target. for sodium (Row 20) :

$$
F 20=(B 20 * E 23 / S B B 23-E 20) /(B 20 * A E B 23 / \$ B \$ 23)
$$

where $B 20=T 4 b k 3.5 \% \mathrm{~N}_{2} \mathrm{O}$ in reneger by feed andy sis

$$
\begin{aligned}
& \text { E2O - Test } 4 \text { lath } \% \mathrm{Na}_{2}
\end{aligned}
$$

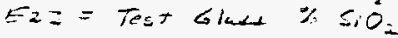

CoG - Same as column F except tostplan rather than curalysis of feed is used to set feet tier set values $(6-110)$

$$
620=(D 20 * \$ E \$=3 / \$ D S=2-E 20) /\left(1020 * \$ E t_{2} 2 / \$ 2 S=3\right)
$$

col lt Loss estimate based on FeD, other wise some as coll

$$
1420=(\$ B 20 * E \$ 4 / \$ B \$ 14-\$ E 20) /(\$ B 20 * \$ E \$ / 4 / \$ B \$ 14)
$$

where $F=$ Row 14

Col I Loss estimate based on Fo (Ra w14) and Testplun (colo)

$$
I 20=(\$ 020 * \$ E \$ 14 / 0,14-\$ E 20) /(\$ 020 * \$ E \$ 14 / \$ 0 \$ 14)
$$

Col I Loss estimate based on GaO (Row 10) and feed Analysis (col) * Note for convenience t's will be eliminate 2 from here on *

$V J_{20}=(B 20 * E 10 / B 10-E 20) /(B 20 * E 10 / B 10)$

BEST AVAILABLE COPY

\begin{tabular}{l|l} 
Tho & Project \\
L SB WHC 2 Spread sheet Documentation & LLWLASS
\end{tabular}

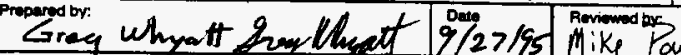

D-12

$9 / 20 / 95$

A1007.115 1000 
WHC-SD-WM-ER-5]7

Revision 0

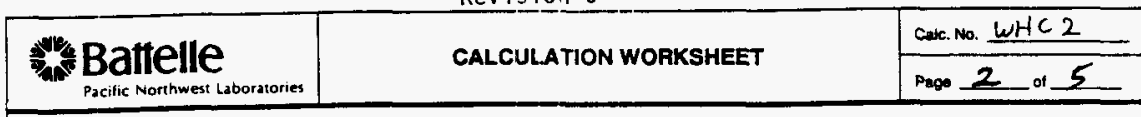

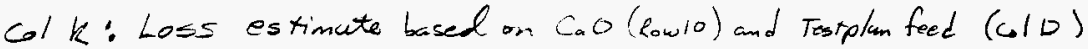

$$
k 20=(D 20 * E 10 / 010-E 20) /(D 20 * E 10 / D 10)
$$

col $L$ Loss estimate bused on $A / 2 \mathrm{O}_{3}\left(R_{0}\right.$ w 6 ) and feed Analysis (C oI)

$$
L 20=(B 20 * E 6 / B 6-E 20) /(B 20 * E 6 / B 6)
$$

6) $M$ Loss exximute baser on $\mathrm{Al}_{2} \mathrm{O}_{3}$ Row is) and Testate (ColD)

$$
M 20=(D 20 * E 6 / 06-E 20) /(020 * E 6 / 106)
$$

Col $N$ Sample analysis adjusted for Tree hugger Compositor on

Assume $1 \mathrm{~kg}$ glass product, tree hugger content is:

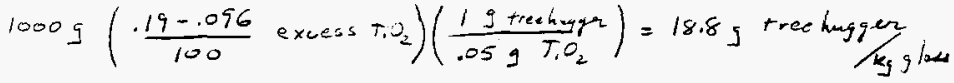

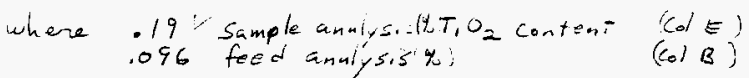

$$
\text { Tresingiger Composition is } \begin{aligned}
& .33 \mathrm{Al}_{2} \mathrm{O}_{3} \\
& .5 \mathrm{C}_{1} \mathrm{O} \\
& .3 \mathrm{~S} \mathrm{~S}_{1} \mathrm{O}_{2} \\
& .05 \mathrm{~T}_{1} \mathrm{O}_{2}
\end{aligned}
$$

Correct masses of affected Compon...te $\mathrm{Al}_{2} \mathrm{O}_{3}=117.1 \mathrm{~g}-18.8(.33)$

$C_{4} O^{3}=65.3-18.8(.45)$

$5 . O_{2}=512.2-18.8(.13)$

$\mathrm{TiO}_{2}=1.9-18.8(.05)$

all other = 10 * Column E or if no ariulysis use feed target

Renormulize to $1 \mathrm{ky}$ product by met-ilyan by $1000 /(1000-18.8)$

Sodium N20 $=E 20 * 10 * 1000 /(1000-18.8)$ genera formula

where 10 - convert col Ex to $1 \mathrm{~kg}$ basis $(100 \%=1 \mathrm{~kg})$

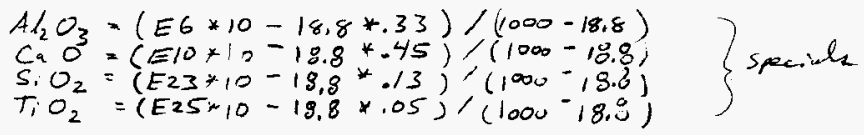

Note: Composition was not normalized. If minor targets are added for non -analyzed species total $\cong 99 \%$

Col Convert from $9 / \mathrm{kg}$ to percent 98.4?

$$
020=N 20 / 10
$$

BEST MWHBBLE COPY

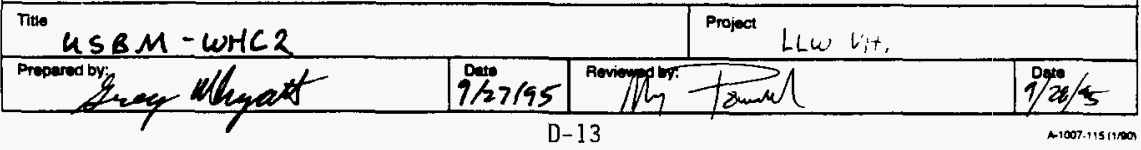


WHC-SD-WM-ER- 517

Revision 0

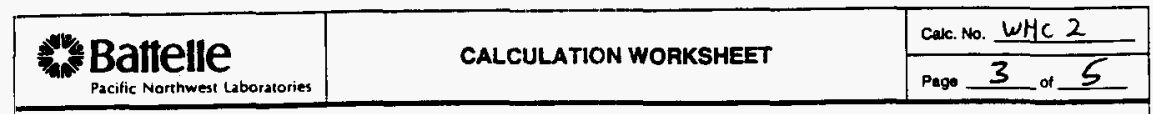

Col P loss estimate based on $\mathrm{SiO}_{2}\left(R_{\text {ow }} 23\right)$ and $B$ feed target (Column B)

$$
=(B 20 * 023 / B 23-020) /\left(B_{20} * 023 / B 23\right)
$$

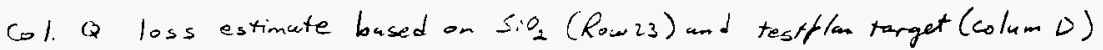

$$
=(D 20 * 023 / 023-020) /(D 20 * 023 / 1023)
$$

col $R$ loss estimate based on $F_{0} O\left(R_{0} / 4\right)$ and feed and lysis (C otB)

$$
=(B 20 * 014 / B 14-020) /(B 20 * 014 / B 14)
$$

Col $S$ loss estimate bused on Foo Row 14 and tesplan $\left(C_{0} / D\right)$

$$
=(020 * 014 / 074-020) /(1220 * 014 / 1014)
$$

Cit $T$ loss estimate bused on $C$ O $(R o w 10)$ and feed andysis (CAB)

$$
=(B 20+010 / B 10-020) /(B 20 * 010 / R 10)
$$

Coll ll loss estimate bused on $\mathrm{CaO}\left(R_{0} \mathrm{w} 10\right)$ and testpkn (Co/10)

$$
=(020 * 010 / 010-020) /(020 * 010 / 010)
$$

col $\mathrm{V}$ loss estimate booed on $\mathrm{Al}_{2} \mathrm{O}_{2}$ (Row 6 ) and food analysis (Col $B$ )

$$
=(B 20 * 06 / \beta 6-020) /(B 20 * 06 / 136)
$$

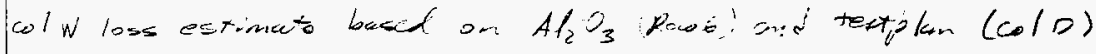

$$
=(1020 * 06 / 06-020) /(020+06 / 06) \text {. }
$$

PEST AMUSABLE COPY

Two $U S B M-W H C 2$

Project

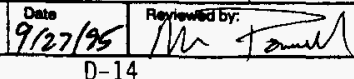

$9 / 20 / 45$ 


\begin{tabular}{|c|c|c|c|c|c|c|c|c|c|c|c|}
\hline$A$ & $B$ & $c$ & $D$ & $E$ & $F$ & $\Leftrightarrow$ & $H$ & $I$ & I & $k$ & $L$ \\
\hline & -1 & -Target Feed & alues-:- & Test Glass & & & & & & & \\
\hline Oxide & B Feed & Tcolumn & Testplan & $\mathrm{WHC2}$ & Tie $=\mathrm{SiO} 2$ & & Tie $=\mathrm{FeO}$ & & $\mathrm{Tie}=\mathrm{CaO}$ & & $\mathrm{T}$ ie $=\mathrm{Al2O} 3$ \\
\hline Compone & Target & intentionally & B feed & & Table 3.5 & Theoretica & Table 3.5 & Theoretical & Table 3.5 & Theoretica & Table 3.5 \\
\hline & Table 3.5 & blank & Target & & B feed & Testplan & B feed & Testplan & $B$ feed & Testplan & B feed \\
\hline & & & & & Target & Target & Target & Target & Target & Target & Target \\
\hline $\mathrm{Al} 2 \mathrm{O} 3$ & $/ 10.28$ & & 10 & $\div \quad 11.71$ & $-22.09 \%$ & $-25.08 \%$ & $-8.94 \%$ & $-11.99 \%$ & $12.78 \%$ & $10.30 \%$ & $0.00 \%$ \\
\hline $\mathrm{B2O} 3$ & 5.01 & & -5.003 & 4.16 & $11.00 \%$ & $11.18 \%$ & $20.59 \%$ & $20.48 \%$ & $36.42 \%$ & $36.31 \%$ & $27.11 \%$ \\
\hline $\mathrm{BaO}$ & 0.003 & & 5 & $\times \quad 0.01$ & $-257.28 \%$ & & $-218.79 \%$ & & $-155.23 \%$ & & $-192.63 \%$ \\
\hline Cl & $\quad 0.38$ & & $<\quad 0.344$ & & & & & & & & \\
\hline $\mathrm{CaO}$ & 5 & & -5.002 & 6.53 & $-39.98 \%$ & $-39.45 \%$ & $-24.90 \%$ & $-24.85 \%$ & $0.00 \%$ & $0.00 \%$ & $-14.65 \%$ \\
\hline $\mathrm{Cr} 2 \mathrm{O} 3$ & $\therefore \quad 0.044$ & & 0.04 & 0.08 & $-94.88 \%$ & $-113.64 \%$ & $-73.89 \%$ & $-91.27 \%$ & $-39.22 \%$ & $-53.20 \%$ & $-59.61 \%$ \\
\hline Cs2O & 0.157 & & -0.144 & & & & & & & & \\
\hline $\mathbf{F}$ & $<0.314$ & & -0.288 & & & & & & & & \\
\hline $\mathrm{FeO}$ & O.811 & & 0.811 & 0.848 & $-12.07 \%$ & $-11.69 \%$ & $0.00 \%$ & $0.00 \%$ & $19.94 \%$ & $19.90 \%$ & $8.21 \%$ \\
\hline $\mathrm{Fe} 2 \mathrm{O} 3$ & & & & 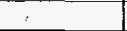 & & & & & & & \\
\hline $\mathrm{K} 2 \mathrm{O}$ & 1.71 & & $<1.585$ & 4.25 & $-166.39 \%$ & $-186.42 \%$ & $-137.69 \%$ & $-156.44 \%$ & $-90.30 \%$ & $-105.40 \%$ & $-118.19 \%$ \\
\hline $\mathrm{MgO}$ & $\quad 0.25$ & & $-\quad 0.248$ & 0.39 & $-67.21 \%$ & $-67.98 \%$ & $-49.19 \%$ & $-50.40 \%$ & $-19.45 \%$ & $-20.46 \%$ & $-36.95 \%$ \\
\hline $\mathrm{MnO} 2$ & $\quad 0.003$ & & $=0.002$ & 0.02 & $-614.56 \%$ & $-968.18 \%$ & $-537.58 \%$ & $-856.37 \%$ & $-410.46 \%$ & $-666.00 \%$ & $-485.25 \%$ \\
\hline$\overline{\mathrm{MoO}} 3$ & $\begin{array}{r}0.159 \\
\end{array}$ & & 0.146 & 0.08 & $46.07 \%$ & $41.47 \%$ & $51.88 \%$ & $47.60 \%$ & $61.47 \%$ & $58.03 \%$ & $55.83 \%$ \\
\hline $\mathrm{Na2O}$ & $\begin{array}{r}19.94 \\
\end{array}$ & & 20.483 & 17.4 & $6.47 \%$ & $9.26 \%$ & $16.55 \%$ & $18.76 \%$ & $33.18 \%$ & $34.93 \%$ & $23.39 \%$ \\
\hline P2O5 & 0.221 & & -0.204 & 0.32 & $-55.20 \%$ & $-67.56 \%$ & $-38.48 \%$ & $-50.02 \%$ & $-10.87 \%$ & $-20.16 \%$ & $-27.11 \%$ \\
\hline $\mathrm{SO} 3$ & $\angle \quad 0.241$ & & $-\quad 0.231$ & 1.01 & $-349.20 \%$ & $-367.04 \%$ & $-300.80 \%$ & $-318.15 \%$ & $-220.89 \%$ & $-234.92 \%$ & $-267.91 \%$ \\
\hline $\mathrm{SiO} 2$ & 54.9 & & 54.712 & 51.22 & $0.00 \%$ & $0.00 \%$ & $10.77 \%$ & $10.47 \%$ & $28.56 \%$ & $28.29 \%$ & $18.10 \%$ \\
\hline Sro & $-\quad 0.113$ & & 0.104 & 0.12 & $-13.82 \%$ & $-23.25 \%$ & $-1.56 \%$ & $-10.35 \%$ & $18.69 \%$ & $11.62 \%$ & $6.77 \%$ \\
\hline $\mathrm{TiO} 2$ & \begin{tabular}{|l} 
\\
\end{tabular} & & 0 & 0.19 & $-112.14 \%$ & & $-89.28 \%$ & & $-51.54 \%$ & & $-73.75 \%$ \\
\hline $\mathrm{ZrO2}$ & 0.005 & & 0 & 0.01 & $-114.37 \%$ & & $-91.27 \%$ & & $-53.14 \%$ & & $-75.58 \%$ \\
\hline t & $\begin{array}{ll}\prime & 0.139\end{array}$ & & $=0.128$ & & & & & & & & \\
\hline unknown? & & & $\prime \quad 0.436$ & & & & & & & & \\
\hline Total & 99.776 & & 99.911 & 98.348 & & & & & & & \\
\hline
\end{tabular}

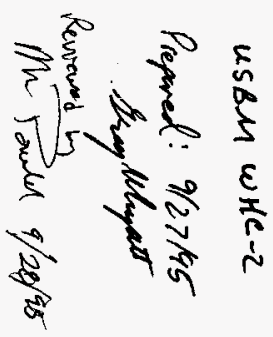




\begin{tabular}{|c|c|c|c|c|c|c|c|c|c|c|c|}
\hline & $M$ & $N$ & 0 & $P$ & $Q$ & $\begin{array}{c}\text { whc2 } \\
R^{*}\end{array}$ & $\begin{array}{l}* \\
5\end{array}$ & $T$ & 4 & $\begin{array}{l}* \\
\psi \\
\end{array}$ & $\begin{array}{c}* \\
W\end{array}$ \\
\hline -......... & & $\mathrm{g} / \mathrm{Kg}$ glass & TiO2 & & & & & & & & \\
\hline Oxide & & ad for THu & Tree-hggr & $\mathrm{Tie}=\mathrm{SiO} 2$ & & Tie $=F e O$ & & Tie $=\mathrm{CaO}$ & & $\mathrm{Tie}=\mathrm{Al} 2 \mathrm{O} 3$ & \\
\hline \multirow[t]{3}{*}{ Compone } & Theoretical & add minors & Adjusted & Table 3.5 & Theoretica| & Table 3.5 & Theoretica & Table 3.5 & Theoretica & Table 3.5 & Theoretical \\
\hline & Testplan & by target & Product & B feed & Testplan & $B$ feed & Testplan & B feed & Testplan & $B$ feed & Testplan \\
\hline & Target & feed analy & $w t \%$ & Target & Target & Target & Target & Target & Target & Target & Target \\
\hline$\overline{A l 203}$ & $0.00 \%$ & $\checkmark 113.02$ & 11.302 & $-16.18 \%$ & $-19.02 \%$ & $-3.23 \%$ & $-6.12 \%$ & $5.11 \%$ & $2.41 \%$ & $0.00 \%$ & $0.00 \%$ \\
\hline $\mathrm{B2O} 3$ & $28.99 \%$ & 42.37 & 4.237 & $10.63 \%$ & $10.81 \%$ & $20.59 \%$ & $20.48 \%$ & $27.00 \%$ & $26.87 \%$ & $23.08 \%$ & $25.07 \%$ \\
\hline $\mathrm{BaO}$ & & 0.10 & 0.010 & $-258.78 \%$ & & $-218.79 \%$ & & $-193.04 \%$ & & $-208.81 \%$ & \\
\hline \multicolumn{12}{|l|}{\begin{tabular}{|l|}
$\mathrm{BaO}$ \\
$\mathrm{Cl}$ \\
\end{tabular}} \\
\hline $\mathrm{CaO}$ & $-11.48 \%$ & 57.93 & 5.793 & $-22.43 \%$ & $-21.96 \%$ & $-8.79 \%$ & $-8.74 \%$ & $0.00 \%$ & $0.00 \%$ & $-5.38 \%$ & $-2.47 \%$ \\
\hline $\mathrm{Cr} 2 \mathrm{O} 3$ & $-70.79 \%$ & 0.81 & 0.081 & $-95.70 \%$ & $-114.53 \%$ & $-73.89 \%$ & $-91.27 \%$ & $-59.84 \%$ & $-75.90 \%$ & $-68.44 \%$ & $-80.24 \%$ \\
\hline \multicolumn{12}{|l|}{$\frac{\mathrm{Cr} 2 \mathrm{O}}{\mathrm{C} 2 \mathrm{O}}$} \\
\hline \multicolumn{12}{|l|}{\begin{tabular}{|l}
$\mathrm{Cs} 2 \mathrm{O}$ \\
$\mathrm{F}$ \\
\end{tabular}} \\
\hline $\mathrm{FeO}$ & $10.71 \%$ & 8.64 & 0.864 & $-12.54 \%$ & $-12.16 \%$ & $0.00 \%$ & $0.00 \%$ & $8.08 \%$ & $8.04 \%$ & $3.13 \%$ & $5.77 \%$ \\
\hline \multicolumn{12}{|c|}{ 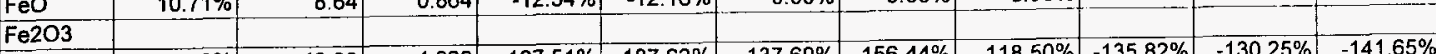 } \\
\hline $\mathrm{K} 2 \mathrm{O}$ & $-128.98 \%$ & 43.29 & 4.329 & $-167.51 \%$ & $-187.62 \%$ & $-137.69 \%$ & $-156.44 \%$ & $-118.50 \%$ & $-135.82 \%$ & $-130.25 \%$ & $-141.65 \%$ \\
\hline $\mathrm{MgO}$ & $-34.29 \%$ & 3.97 & 0.397 & $-67.91 \%$ & $-68.68 \%$ & $-49.19 \%$ & $-50.40 \%$ & $-37.14 \%$ & $-38.30 \%$ & $-44.52 \%$ & $-41.72 \%$ \\
\hline $\mathrm{MnO} 2$ & $-753.97 \%$ & 0.20 & 0.020 & $-617.55 \%$ & $-972.64 \%$ & $-537.58 \%$ & $-856.37 \%$ & $-486.08 \%$ & $-779.48 \%$ & $-517.62 \%$ & $-801.19 \%$ \\
\hline $\mathrm{MoO} 3$ & $53.21 \%$ & 0.81 & 0.081 & $45.85 \%$ & $41.23 \%$ & $51.88 \%$ & $47.60 \%$ & $55.77 \%$ & $51.81 \%$ & $53.39 \%$ & $50.62 \%$ \\
\hline $\mathrm{Na} 2 \mathrm{O}$ & $27.46 \%$ & 177.23 & 17.723 & $6.08 \%$ & $8.88 \%$ & $16.55 \%$ & $18.76 \%$ & $23.29 \%$ & $25.29 \%$ & $19.16 \%$ & $23.44 \%$ \\
\hline $\mathrm{P} 205$ & $-33.96 \%$ & 3.26 & 0.326 & $-55.85 \%$ & $-68.26 \%$ & $-38.48 \%$ & $-50.02 \%$ & $-27.29 \%$ & $-37.96 \%$ & $-34.14 \%$ & $-41.36 \%$ \\
\hline 503 & $-273.38 \%$ & 10.29 & 1.029 & $-351.08 \%$ & $-368.99 \%$ & $-300.80 \%$ & $-318.15 \%$ & $-268.43 \%$ & $-284.53 \%$ & $-288.25 \%$ & $-294.03 \%$ \\
\hline $\mathrm{SiO} 2$ & $20.05 \%$ & $\sqrt{519.52}$ & 51.952 & $0.00 \%$ & $0.00 \%$ & $11.15 \%$ & $10.84 \%$ & $18.32 \%$ & $18.01 \%$ & $13.93 \%$ & $15.98 \%$ \\
\hline Sro & $1.46 \%$ & 1.22 & 0.122 & $-14.30 \%$ & $-23.77 \%$ & $-1.56 \%$ & $-10.35 \%$ & $6.64 \%$ & $-1.48 \%$ & $1.62 \%$ & $-3.98 \%$ \\
\hline TiO2 & & 0.98 & 0.098 & $-7.70 \%$ & & $4.30 \%$ & & $12.03 \%$ & & $7.30 \%$ & \\
\hline ZrO2 & & 0.10 & 0.010 & $-115.27 \%$ & & $-91.27 \%$ & & $-75.82 \%$ & & $-85.29 \%$ & \\
\hline \multicolumn{12}{|l|}{1} \\
\hline \multicolumn{12}{|l|}{ unknown? } \\
\hline Total & & 983.75 & 98.38 & & & & & & & & \\
\hline
\end{tabular}




$$
\begin{gathered}
\text { WHC-SD-WM-ER- } 517 \\
\text { Revision } 0 \\
\hline
\end{gathered}
$$

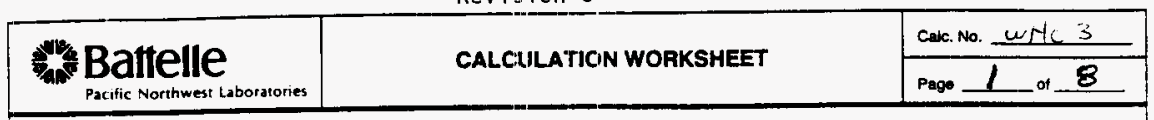

Documentation of WHC 3 spreadsheet:

$$
\text { Fie C: \LLWGlass Vendors IUSBM \WHC3 DF-B. XLS }
$$

The cells are documented only where they contribute to reported values. Other calculations are information only.

f Col. B Table 3.5 of USBM report on WHCl, analysis of B feed (entered) Col. D Target glass composition based on tectpkn (intruded) Cole Analysis of WHC3-spIBM(1) (entered)

$\checkmark$ rot $F$ excess COO and Fe O subtracted, missing analyzed $\mathrm{Sr}_{r}+I$ set e teatphan

$$
\begin{aligned}
& C_{4} O=E 24 * 5.002 / 54_{1}>12 \text {. for normalization purposed only }
\end{aligned}
$$

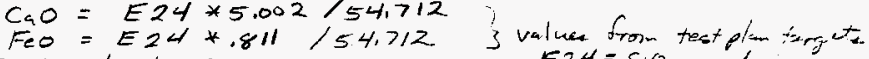

$$
\begin{aligned}
& \text { Total calculation in }=30 \quad E 24=\mathrm{SiO}_{2} \text { andlysi= }
\end{aligned}
$$

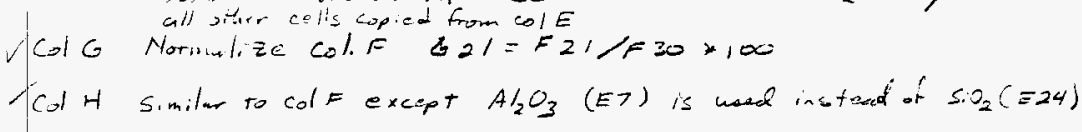

$$
\begin{aligned}
& \operatorname{cul}=E>+5.002 / 10 \\
& F_{e O}=E 7 * \text { aI } / 1 \\
& \text { total H30 } \\
& \text { other til copied from } c / E \\
& S_{r}+I \text { set at test plan tarych for rerenclizintion }
\end{aligned}
$$

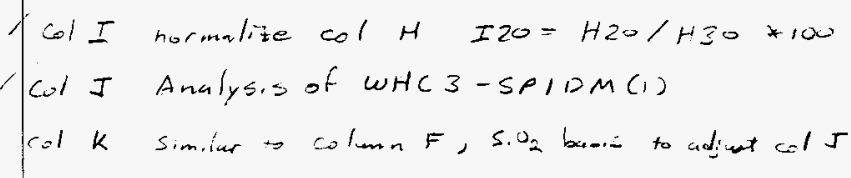

$$
\begin{aligned}
& C_{5} O=J 24 * 5002 / 54.712 \\
& F=0=J 24 \cdot .611 / 5+.7= \\
& \text { total in } K 30 \\
& \text { other lithe from I } \\
& \text { sr t I at te-tplan trivet }
\end{aligned}
$$

C.I L Normalize col k result $L 20=k 20 / k 30 * 100$

Col $M$ similar to col $K$ except $A / O_{3}$ basis (Row 7 )

$$
\begin{aligned}
& C_{4 O}=J 7 * 5,002 / 54.712 \\
& E_{0 O}=J 7 *, 811 / 10
\end{aligned}
$$

BEST ANALABLE COPY

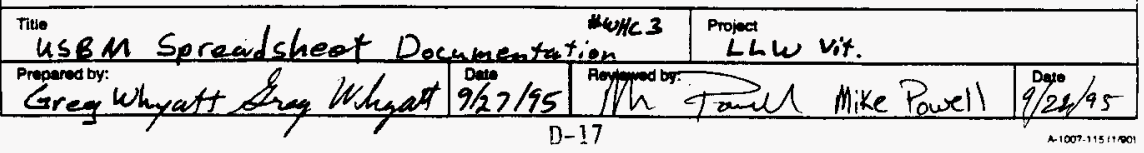


WHC-SD-WM-ER-5]7

Revision 0

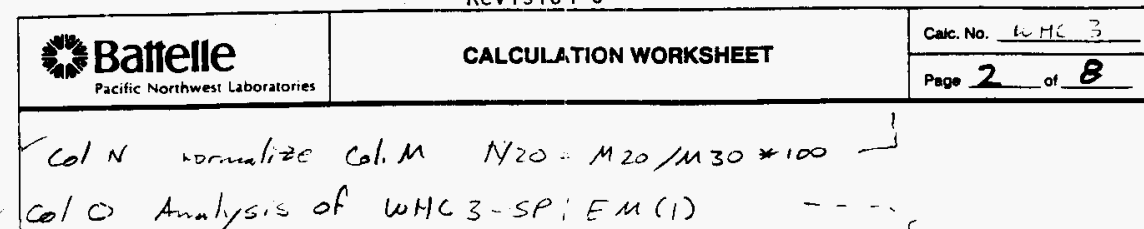

Colc Anulysis of WHC 3-SP:EM(1)

col $\mathrm{P}$ as in $\mathrm{F}$, sion basis, colo

$$
\begin{aligned}
& C_{4} O=024 * 5.002 / 54,7 / 2 \\
& F_{e} O=024 * .811 / 54,712
\end{aligned}
$$

$\operatorname{col} Q$ hormmlize $Q 21=021 / 1030+100$

Col $R$ in $H: \mathrm{Al}_{2} \mathrm{O}_{3}$ basis, ClO

$$
\begin{aligned}
& C . O=0>* 5,002 / 10 \\
& \angle O=07 * 1811 / 10
\end{aligned}
$$

Cols normulize $R: 520=R 20 / R 30 * 100$

col $T$

$$
\text { analysis of WHC3-SPIAM(1) }
$$

$\cot 4$

$$
\begin{aligned}
& \therefore \text { in }=: S_{1} O_{2}, c_{0} l T \\
& c_{a} O=T 24 * 5.002 / 5 \% 1712 \\
& F_{e 0}=T 24 * .811 / 54.712
\end{aligned}
$$

Golv normalize cl 4 : $v_{20}=420 / 430 * 100$

colw as in $H: A / 2 O_{3}$ Col $T$

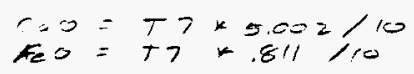

Colx hormalize wl w

$\checkmark$ Col Aw Caiculate losses for Sample wHC3-SPI BMC) by calaluting

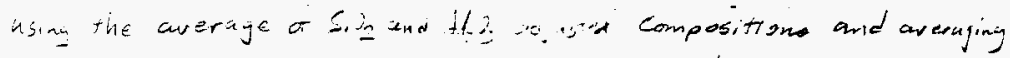

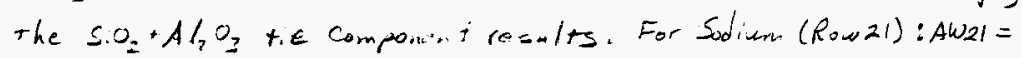

$$
\begin{aligned}
& (((B 21 *(G 24+I 24) / 2 / B 24-(G 21+I 21) / 2) /(B 21 *(G 24+I 24) / 2 / B 24)) \\
& +((C B 21 *(G)+I 7) / 2 / B>-(G 21+I 21) / 2) /(B 21 *(G)+I 7) / 2 / B>)) \\
& / 2
\end{aligned}
$$

BRST AVALABLE COPY

whene $\mathrm{T}=\mathrm{Al}_{2} \mathrm{O}_{3}, 21=\mathrm{Na}_{2} \mathrm{O}, 24=5 . \mathrm{O}_{2}, \mathrm{G}^{2}$ normulized composition adj.

for $\mathrm{CaO}_{1} \mathrm{feO}_{\mathrm{O}}$ using $\mathrm{SiO}_{2}, \mathrm{I}^{\prime}=$ norm. comp adj using $\mathrm{Al}_{2} \mathrm{O}_{3}$

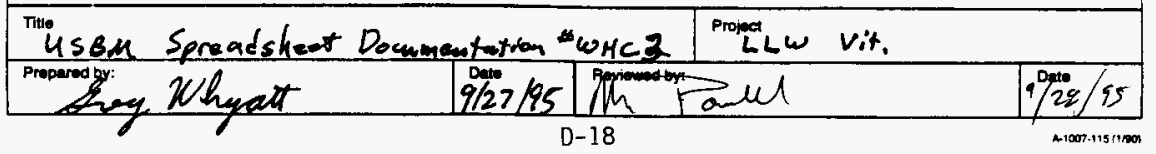




$$
\begin{aligned}
& W H C-S D-W^{\prime} N-E R-5: 7 \\
& \text { Revision } 0
\end{aligned}
$$

Battelle

CALCULATION WORKSHEET

Pacific Northwest Laboratories

Page 3 of 8

$\checkmark$ Col AY: Same as Aw except $\mathrm{Sin}_{2}$ adjusted $=L \quad \mathrm{Al}_{2} \mathrm{O}_{3}$ adjusted $=N$

$$
\begin{gathered}
=(((B 21 *(L 24+N 24) / 2 / B 24-(L 21+N 21) / 2) /(B 21+(L 24+N 24) / 2 / B 24))+ \\
((B 2) *(L 7+N>) / 2 / B>-(L 21+N 21) / 2) /(B B 2 / *(L 7+N 7) / 2 / B 7)) \\
) / 2
\end{gathered}
$$

$\frac{C O \mid B A}{B A 21=}$ : Same us $A w$ except $\quad \mathrm{SIO}_{2}$ adjusted $=Q \quad \mathrm{~A} / 2 \mathrm{O}_{3}$ adjust $=S$

$$
\begin{aligned}
& (((B 2 / *(Q 24+524) / 2 / B 24-(Q 21+521) / 2) /(B 21 *(Q 24+524) / 2 / B 24))+ \\
& ((B 2 / *(Q 7+57) / 2 / B 2-(Q 21+521) / 2) /(B 21 *(Q 7+57) / 2 / B>)) \\
& \quad) / 2
\end{aligned}
$$

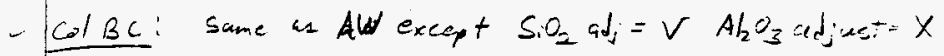

$$
\begin{aligned}
& B C 21=\left(\left(\left(B 321 *\left(V_{24}+x=4\right) / 2 / B=4-\left(V_{21}+x_{2} 1\right) / 2\right) /\left(1221 *\left(V 24+x_{24}\right) / 2 / B 24\right)\right)+\right. \\
& ((B 21 *(v 7+x>) / 2 / B 7-(121+; 2) / 2 ; /(B 21-(17 ; \div 7) / 2 / B 7)) \\
& \text { ) } 1 / 2
\end{aligned}
$$

$$
\begin{aligned}
& \text { Sheet } 2 \operatorname{col} B=\text { sheet } \operatorname{col} A w \\
& \text { shit Col } C=\text { herl col AY } \\
& \text { shr } 2 \text { col } D=\operatorname{sit} 1 \text { ar } B A
\end{aligned}
$$

(Sr, I value deletion)

Reviewers comments:

RECT AVALABIE COPY

*) Cheek marks identify cells, columns, and formulas verified
by the reviewer

*) Some of the input glass concentration value: Shown on the attociued spreadsheet do not match the values given in the user report. The difference is due solely to the cell formats used to display the values in
Excl. The values are correct.

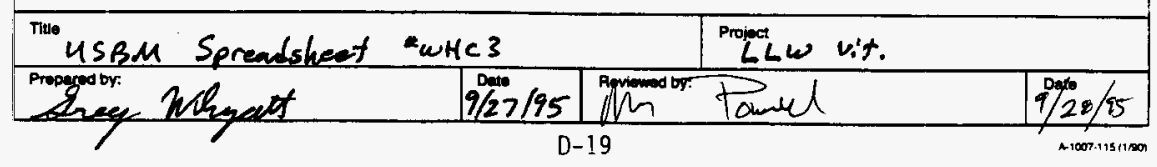




\begin{tabular}{|c|c|c|c|c|c|c|c|c|c|c|c|c|c|c|c|}
\hline & A & B & C & D & $\bar{E}$ & $\mathbf{F}$ & $\mathbf{G}$ & $\mathrm{H}$ & $I$ & $\mathbf{J}$ & $\mathrm{K}$ & $\mathbf{L}$ & $\mathbf{M}$ & $\mathbf{N}$ & \\
\hline 2 & & -Ta & arget Feed Valu & yes-.. & Test Glass & $\mathrm{SiO} 2$ & & $\mathrm{Al2O3}$ & & Test Glass & $\mathrm{SiO} 2$ & & $\mathrm{~A} \mid 203$ & & \\
\hline 3 & Oxide & B Feed & ? & Testplan & WHC3-SP1BM(1) & Ratio on $\mathrm{CaO}$ & & Ratio on $\mathrm{CaO}$ & & WHC3-SPIDM(1) & Ratio on $\mathrm{CaO}$ & & Ratio on $\mathrm{Ca}$ & & \\
\hline 4 & Component & Target & normalized & B feed & 19.55 & and $\mathrm{FeO}$ & & and $\mathrm{FeO}$ & & $20: 42$ & and FeO & & and $\mathrm{FeO}$ & & \\
\hline 5 & & Table 3.5 & Target & Target & analysis & NA entries & & NA entries & & analysis & NA entries & & NA entries & & \\
\hline 6 & & & & & & on TP target & Normalized & on TP target & Normalized & & on TP target & Normalized & on TP targe & Normalized & \\
\hline$\overline{7}$ & $\widehat{\mathrm{Al} 2 \mathrm{O3}}$ & 10.28 & 10.3030789 & 10 & 10.22 & 10.22 & 10.72 & 10.22 & 10.70 & 10.05 & 10.05 & 10.35 & 10.05 & 10.36 & \\
\hline 8 & $\mathrm{B203}$ & 5.01 & 5.021247595 & 5.003 & 4.02 & 4.02 & 4.22 & 4.02 & 4.21 & 4.25 & 4.25 & $4 . \overline{38}$ & 4.25 & 4.38 & \\
\hline 9 & $\mathrm{BaO}$ & 0.003 & 0.003006735 & 0 & 0.01 & 0.01 & 0.01 & 0.01 & 0.01 & 0.01 & 0.01 & 0.01 & 0.01 & 0.01 & \\
\hline 10 & CI & 0.38 & 0.380853111 & 0.344 & 0.013 & 0.013 & 0.01 & 0.013 & 0.01 & 0.01 & 0.01 & 0.01 & 0.01 & 0.01 & \\
\hline 11 & $\mathrm{CaO}$ & 5 & 5.011225144 & 5.002 & 7.25 & 14.987189282 & 5.23 & V 5.112044 & -5.35 & 7.81 & 5.08501316 & 5.24 & 5.02701 & 5.18 & \\
\hline 12 & $\mathrm{Cr}_{2} \mathrm{O}^{\prime}$ & $\therefore \quad 0.044$ & 0.044098781 & 0.04 & 0.08 & 0.08 & 0.08 & 0.08 & 0.08 & 0.06 & 0.06 & 0.06 & 0.06 & 0.06 & \\
\hline 13 & Cs2O & -0.157 & 0.15735247 & 0.144 & 0.099 & 0.099 & 0.10 & 0.099 & 0.10 & $0.096 \quad 0.10$ & 0.10 & 0.10 & 0.10 & 0.10 & \\
\hline 14 & $F$ & $r \quad 0.314$ & 0.314704939 & 0.288 & 0.001 & 0.001 & 0.00 & 0.001 & 0.00 & 1.1 & 0.00 & 0.00 & 0.00 & 0.00 & \\
\hline 15 & FeO & $r \quad 0.811$ & 0.812820718 & 0.811 & 1.4 & 0.808598662 & 0.85 & 0.828842 & 0.87 & 1.38 & 0.824459351 & 0.85 & 0.815055 & 0.84 & \\
\hline 16 & $\mathrm{Fe} 2 \mathrm{O3}$ & & & & 0 & 0 & 0.00 & 0 & 0.00 & 0.00 & 0.00 & 0.00 & 0.00 & 0.00 & \\
\hline 17 & K2O & 1.71 & 1.713838999 & 1.585 & 1.25 & 1.25 & 1.31 & 1.25 & 1.31 & 1.27 & 1.27 & 1.31 & 1.27 & 1.31 & \\
\hline 18 & $\mathrm{MgO}$ & 0.25 & 0.250561257 & 0.248 & 0.42 & 0.42 & 0.44 & 0.42 & 0.44 & 0.39 & 0.39 & 0.40 & 0.39 & 0.40 & \\
\hline 19 & $\mathrm{MnO} 2$ & $r \quad 0.003$ & 0.003006735 & 0.002 & 0.02 & 0.02 & 0.02 & 0.02 & 0.02 & 0.02 & 0.02 & $\overline{0} . \overline{0} \overline{2}$ & 0.02 & $\overline{0.02}$ & 贾 in \\
\hline 20 & MoO3. & 0.159 & 0.15935696 & 0.146 & 0.077 & 0.077 & 0.08 & 0.077 & 0.08 & 0.08 & 0.08 & 0.08 & 0.08 & 0.08 & $\leq 9$ \\
\hline 21 & $\mathrm{Na2O}$ & 19.94 & 19.98476588 & 20.483 & 18.2 & 18.2 & 19.09 & 18.2 & 19.06 & 18.74 & 18.74 & 19.30 & 18.74 & 19.32 & $\ddot{n}$ \\
\hline 22 & $\mathbf{P 2 0 5}$ & 0.221 & 0.221496151 & 0.204 & 0.12 & 0.12 & 0.13 & 0.12 & 0.13 & 0.10 & 0.10 & 0.10 & 0.10 & 0.10 & $0 \hat{1}$ \\
\hline 23 & SO3 & 0.241 & 0.241541052 & 0.231 & 0.02 & 0.02 & 0.02 & 0.02 & 0.02 & 0.02 & $0.0 \overline{2}$ & 0.02 & 0.02 & 0.02 & 罗 \\
\hline 24 & $\mathrm{SiO} 2$ & 54.9 & 55.02325208 & 54.712 & $\overline{54.55}$ & 54.55 & 57.21 & 54.55 & 57.12 & 55.62 & 55.62 & 57.30 & 55.62 & 57.33 & 01 \\
\hline 25 & Sro & $\quad 0.113$ & 0.113253688 & 0.104 & & 0.104 & 0.11 & 0.104 & 0.11 & & 0.104 & 0.11 & 0.104 & 0.11 & $=$ \\
\hline 26 & TिIO2 & 0.096 & 0.096215523 & 0 & 0.19 & 0.19 & 0.20 & 0.19 & 0.20 & 0.19 & 0.19 & 0.20 & 0.19 & 0.20 & \\
\hline 27 & $\mathrm{ZrO} 2$ & 0.005 & 0.005011225 & o & 0.04 & 0.04 & 0.04 & 0.04 & 0.04 & 0.03 & 0.03 & 0.03 & 0.03 & 0.03 & \\
\hline 28 & 1 & $\div \quad 0.139$ & 0.139312059 & 0.128 & & $0.12 \overline{8}$ & 0.13 & 0.128 & 0.13 & & 0.128 & 0.13 & 0.128 & 0.13 & \\
\hline 29 & unknown? & & & 0.436 & & & & & & & & & & & \\
\hline 30 & Total & 99.776 & 100 & 99.911 & 97.98 & 95.35778794 & 100 & 95.502886 & 100 & 100.125 & 97.07647251 & 100 & 97.009065 & 100 & \\
\hline
\end{tabular}

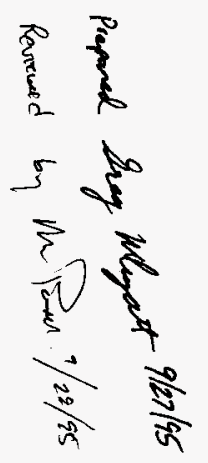




\begin{tabular}{|c|c|c|c|c|c|c|c|c|c|c|c|c|c|}
\hline & A & 0 & $\overline{\mathbf{P}}$ & $\mathbf{Q}$ & $\mathbf{R}$ & 5 & $T$ & $\mathbf{U}$ & v & $\mathbf{w}$ & $x$ & $Y$ & $z$ \\
\hline 2 & & Test Glass & $\mathrm{SiO} 2$ & & $\mathrm{Al} / 2 \mathrm{O} 3$ & & Test Glass & $\mathrm{SiO} 2$ & & Al2O3 & & Test Glass & \\
\hline 3 & Oxide & WHC3-SP1EM(1) & Ratio on $\mathrm{CaO}$ & & Ratio on $\mathrm{CaO}$ & & WHC3-SPIAM(1) & Ratio on CaO & & Ratio on CaO & & WHC3-SP1E & $\mathrm{BM}(1)$ \\
\hline 4 & Component & $21: 19$ & and $\mathrm{FeO}$ & & and $\mathrm{FeO}$ & & $20: 42$ & and $\mathrm{FeO}$ & & and $\mathrm{FeO}$ & & $19: 55$ & \\
\hline 5 & & analysis & NA entries & & NA entries & & anaiysis & NA entries & & NA entries & & & \\
\hline$\frac{2}{6}$ & & & on TP target & Normalized & on TP target & Normalized & & on IP target & Nomalized & on TP target & Normalized & $\mathrm{SiO} 2$ & $\mathrm{~A} \mid 203$ \\
\hline$\frac{6}{7}$ & Al2O3 & -9.79 & 9.79 & 10.89 & 9.79 & 10.87 & 9.67 & 9.67 & 10.40 & 9.67 & 10.38 & 10.72 & 10.70 \\
\hline 8 & $\mathrm{B2O3}$ & -3.73 & 3.73 & 4.15 & 3.73 & 4.14 & 4.02 & 4.02 & 4.32 & 4.02 & 4.32 & 4.22 & 4.21 \\
\hline 9 & $\overline{\mathrm{BaO}}$ & 0.01 & 0.01 & 0.01 & 0.01 & 0.01 & -0.01 & 0.01 & 0.01 & 0.01 & 0.01 & 0.01 & 0.01 \\
\hline 10 & Cl & $.005 \cdot 0.01$ & 0.01 & 0.01 & 0.01 & 0.01 & $\because \quad 0.01$ & 0.01 & 0.01 & 0.01 & 0.01 & 0.01 & 0.01 \\
\hline 11 & $\mathrm{CaO}$ & -13.43 & 4.791541527 & 5.33 & 4.896958 & 5.44 & $r \quad 12.34$ & 4.733030048 & 5.09 & 4836934 & 5.19 & 5.23 & $\overline{5.35}$ \\
\hline 12 & $\mathrm{Cr} 203$ & 0.07 & 0.07 & 0.08 & 0.07 & 0.08 & -0.07 & 0.07 & 0.08 & $0 . \overline{0} 7$ & 0.08 & 0.08 & 0.08 \\
\hline 13 & $\mathrm{Cs} 2 \mathrm{O}$ & $\begin{array}{ll}082 & 0.08\end{array}$ & 0.08 & 0.09 & 0.08 & 0.09 & .0170 .10 & 0.10 & 0.10 & 0.10 & 0.10 & 0.10 & 0.10 \\
\hline 14 & $\bar{F}$ & 0.0020 .00 & 0.00 & 0.00 & 0.00 & 0.00 & $.002 \quad 0.00$ & 0.00 & 0.00 & 0.00 & 0.00 & 0.00 & 0.00 \\
\hline 15 & FeO & $-\quad 1.42$ & 0.776877285 & 0.86 & 0.793969 & 0.88 & -1.13 & 0.767390518 & 0.83 & 0.784237 & 0.84 & 0.85 & 0.87 \\
\hline 16 & $\mathrm{Fe} 2 \mathrm{O} 3$ & 0.00 & 0.00 & 0.00 & 0.00 & 0.00 & $=0.00$ & 0.00 & 0.00 & 0.00 & 0.00 & 0.00 & 0.00 \\
\hline 17 & $\mathrm{~K} 2 \mathrm{O}$ & 1.19 & 1.19 & 1.32 & 1.19 & 1.32 & 1.25 & 1.25 & 1.34 & 1.25 & 1.34 & 1.31 & 1.31 \\
\hline 18 & $\mathrm{MgO}$ & 0.47 & 0.47 & 0.52 & 0.47 & 0.52 & 0.41 & 0.41 & 0.44 & 0.41 & 0.44 & 0.44 & 0.44 \\
\hline 19 & $\mathrm{MnO} 2$ & .024 & 0.02 & 0.03 & 0.02 & 0.03 & 0.02 & 0.02 & 0.02 & 0.02 & 0.02 & 0.02 & 0.02 \\
\hline 20 & $\mathrm{MoO3}$ & .076 & 0.08 & 0.08 & 0.08 & 0.08 & 0.11 & 0.11 & 0.11 & 0.11 & 0.11 & 0.08 & 0.08 \\
\hline 24 & $\mathrm{Na2O}$ & 15.91 & 15.91 & 17.69 & 15.91 & 17.67 & 19.55 & 19.55 & 21.02 & 19.55 & 20.99 & 19.09 & 19.06 \\
\hline 22 & P2O5 & 0.09 & 0.09 & 0.10 & 0.09 & 0.10 & 0.09 & 0.09 & 0.10 & 0.09 & 0.10 & 0.13 & 0.13 \\
\hline 23 & $\mathrm{so3}$ & 0.01 & 0.01 & 0.01 & 0.01 & 0.01 & 0.01 & 0.01 & 0.01 & 0.01 & 0.01 & 0.02 & 0.02 \\
\hline 24 & $\mathrm{SiO} 2$ & 52.41 & 52.41 & 58.29 & 52.41 & 58.21 & 51.77 & 51.77 & 55.66 & 51.77 & 55.58 & 57.21 & 57.12 \\
\hline 25 & Sro & 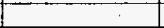 & 0.10 & 0.12 & 0.10 & 0.12 & & 0.10 & 0.11 & 0.10 & 0.11 & 0.11 & 0.11 \\
\hline 28 & TiO2 & 0.21 & 0.21 & 0.23 & 0.21 & 0.23 & 0.17 & 0.17 & 0.18 & 0.17 & 0.18 & 0.20 & 0.20 \\
\hline 27 & ZrO2 & 0.04 & 0.04 & 0.04 & 0.04 & 0.04 & 0.03 & 0.03 & 0.03 & 0.03 & 0.03 & 0.04 & 0.04 \\
\hline 28 & 1 & & 0.13 & 0.14 & 0.13 & 0.14 & & 0.13 & 0.14 & $0 . \overline{13}$ & 0.14 & 0.13 & 0.13 \\
\hline 20 & unknown? & & & & & & & & & & & 0.00 & 0.00 \\
\hline 30 & Total & 798.969 & 89.91941881 & 100 & 90.041927 & 100 & 100.754 & 93.01642057 & 100 & 93.137171 & 100 & 100.00 & 100.00 \\
\hline
\end{tabular}

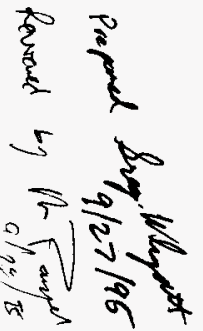




\begin{tabular}{|c|c|c|c|c|c|c|c|c|c|c|c|c|c|c|c|c|}
\hline & & A & AA & $\overline{\mathrm{AB}}$ & AC & AD & $\frac{1}{A E}$ & AF & AG & $\mathbf{A H}$ & $\overline{A l}$ & AJ & $\overline{A K}$ & $\overline{A L}$ & $\overline{A M}$ & $\overline{A N}$ \\
\hline & 2 & & Test Glass & & Test Glass & & Test Glass & & & & & & & \multicolumn{2}{|c|}{ Fractional Volatility } & Final Glas \\
\hline & 3 & Oxide & \multicolumn{2}{|c|}{ WHC3-SP1DM(1) } & WHC3-SP1E & $E M(1)$ & \multicolumn{2}{|c|}{ WHC3-SP1AM(1) } & 4 sampie & $\mathrm{Tie}=\mathrm{SiO}_{2}$ & & Tie $=\mathrm{Al} 2 \mathrm{O} 3$ & & & & Test Glas \\
\hline & 1 & Component & $20: 42$ & & $21: 19$ & & $20: 42$ & & Avg value & Table 3.5 & Theoretica & Table 3.5 & Theoretical & Avg loss & Avg loss & WHC3-SP \\
\hline & 5 & & & & & & & & both methods & B feed & Testplan & $B$ feed & Testplan & $\%$ of & $\%$ of glass & $20: 42$ \\
\hline & 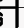 & & $\mathrm{SiO} 2$ & $\mathrm{Al} 2 \mathrm{O3}$ & SiO2 & $\overline{\mathrm{Al} 2 \mathrm{O} 3}$ & $\overline{\mathrm{SiO} 2}$ & $\overline{\mathrm{A} \mid 2 \mathrm{O} 3}$ & & Target & Target & Target & Target & oxide & tot mass & $\overline{\mathrm{SiO} 2}$ \\
\hline 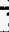 & 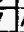 & $\overline{\mathrm{Al} 2 \mathrm{O} 3}$ & 10.35 & 10.36 & 10.89 & 10.87 & 10.40 & 10.38 & $\checkmark 10.58$ & $0.99 \%$ & $-1.44 \%$ & $0.00 \%$ & $0.00 \%$ & $-0.11 \%$ & -0.01138 & 10.40 \\
\hline 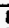 & 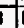 & $\mathrm{B} 2 \mathrm{O} 3$ & 4.38 & 4.38 & 4.15 & 4.14 & 4.32 & 4.32 & $\checkmark 4.26$ & $18.15 \%$ & $18.31 \%$ & $17.33 \%$ & $19.47 \%$ & $18.32 \%$ & 0.916973 & $4 . \overline{32}$ \\
\hline & 5 & $\mathrm{BaO}$ & 0.01 & 0.01 & 0.01 & 0.01 & 0.01 & 0.01 & 0.01 & $-241.73 \%$ & NA & $-245.14 \%$ & NA & $-243.44 \%$ & -0.0073 & 0.01 \\
\hline & ] & $\overline{\mathrm{Cl}}$ & 0.01 & 0.01 & 0.01 & 0.01 & 0.01 & 0.01 & 0.01 & $97.45 \%$ & $97.20 \%$ & $97.43 \%$ & $97.24 \%$ & $97.33 \%$ & \begin{tabular}{|l|}
0.352335 \\
\end{tabular} & 0.01 \\
\hline & 1 & $\mathrm{CaO}$ & 5.24 & 5.18 & 5.33 & 5.44 & 5.09 & 5.19 & 5.26 & $-1.10 \%$ & $-0.72 \%$ & $-2.11 \%$ & $0.71 \%$ & $-0.81 \%$ & -0.04032 & 5.09 \\
\hline 1 & 2 & $\mathrm{Cr} 203$ & 0.06 & 0.06 & 0.08 & 0.08 & 0.08 & 0.08 & 0.07 & $-63.20 \%$ & $-78.90 \%$ & $-64.82 \%$ & $-76.37 \%$ & $-70.82 \%$ & -0.02975 & 0.08 \\
\hline & 3 & $\mathrm{Cs2O}$ & 0.10 & 0.10 & 0.09 & 0.09 & 0.10 & 0.10 & 0.10 & $39.05 \%$ & $33.77 \%$ & $38.44 \%$ & $34.71 \%$ & $36.49 \%$ & 0.054924 & 0.10 \\
\hline & 4 & $\mathbf{F}$ & 0.00 & 0.00 & 0.00 & 0.00 & 0.00 & 0.00 & 0.00 & $99.51 \%$ & $99.46 \%$ & $99.50 \%$ & $99.47 \%$ & $99.49 \%$ & 0.299451 & 0.00 \\
\hline & 6 & $\overline{\mathrm{FeO}}$ & 0.85 & 0.84 & 0.86 & 0.88 & 0.83 & 0.84 & 0.85 & $-1.06 \%$ & $-0.72 \%$ & $-2.07 \%$ & $0.71 \%$ & $-0.79 \%$ & -0.00637 & 0.83 \\
\hline & 6 & $\mathrm{Fe} 2 \mathrm{O3}$ & 0.00 & 0.00 & 0.00 & 0.00 & 0.00 & 0.00 & 0.00 & NA & NA & NA & NA & NA & NA & 0.00 \\
\hline & 7 & $\mathrm{~K} 2 \mathrm{O}$ & 1.31 & 1.31 & 1.32 & 1.32 & 1.34 & 1.34 & 1.32 & $25.71 \%$ & $20.12 \%$ & $24.97 \%$ & $21.25 \%$ & $23.01 \%$ & 0.37911 & 1.34 \\
\hline & 8 & $\mathrm{MgO}$ & 0.40 & 0.40 & 0.52 & 0.52 & 0.44 & 0.44 & 0.45 & $-73.57 \%$ & $-74.37 \%$ & $-75.30 \%$ & $-74.90 \%$ & $-73.79 \%$ & -0.18373 & $0 . \overline{44}$ \\
\hline & 9 & $\mathrm{MnO2}$ & 0.02 & 0.02 & 0.03 & 0.03 & 0.02 & 0.02 & 0.02 & $-610.48 \%$ & $-962.08 \%$ & $-617.57 \%$ & $-947.04 \%$ & $-784.29 \%$ & -0.01961 & 0.02 \\
\hline & 0 & $\mathrm{MoO3}$ & 0.08 & 0.08 & 0.08 & 0.08 & 0.11 & 0.11 & 0.09 & $45.65 \%$ & $41.02 \%$ & $45.11 \%$ & $41.85 \%$ & $43.41 \%$ & 0.066197 & 0.11 \\
\hline & 1 & $\mathrm{Na2O}$ & 19.30 & 19.32 & 17.69 & 17.67 & 21.02 & 20.99 & 19.27 & $7.07 \%$ & $9.85 \%$ & $6.15 \%$ & $11.12 \%$ & $8.55 \%$ & 1.727824 & 21.02 \\
\hline & 2 & P2O5 & 0.10 & 0.10 & 0.10 & 0.10 & 0.10 & 0.10 & 0.11 & $53.71 \%$ & $50.02 \%$ & $53.25 \%$ & $50.73 \%$ & $51.93 \%$ & 0.110345 & 0.10 \\
\hline & 3 & SO3 & 0.02 & 0.02 & 0.01 & 0.01 & 0.01 & 0.01 & 0.02 & $93.67 \%$ & $93.42 \%$ & $93.61 \%$ & $93.51 \%$ & $93.55 \%$ & 0.220789 & 0.01 \\
\hline & 4 & $\mathrm{SiO2}$ & 57.30 & 57.33 & 58.29 & 58.21 & 55.66 & 55.58 & 57.09 & $0.00 \%$ & $0.00 \%$ & $-1.00 \%$ & $1.42 \%$ & $0.10 \%$ & 0.057364 & 55.66 \\
\hline & 6 & $\mathrm{SrO}+\mathrm{t}$ & $6,0,11$ & 0.11 & 0.12 & 0.12 & 0.11 & 0.11 & 0.11 & NÁ & NA & NA & $\overline{N A}$ & NA & NA & 0.11 \\
\hline & 6 & $\overline{\mathrm{T}} \mathrm{O} 2 \mathrm{~K}$ & 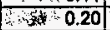 & 00.20 & 0.23 & 0.23 & 0.18 & 0.18 & 0.20 & $-103.09 \%$ & NA & $-105.12 \%$ & NA & $-104.10 \%$ & -0.09994 & 0.18 \\
\hline & 7 & $\mathrm{ZrO2}, 34$ & कर 0.03 & 0.03 & 0.04 & 0.04 & 0.03 & 0.03 & 0.04 & $-618.95 \%$ & NA & $-626.12 \%$ & NA & $-622.53 \%$ & -0.03113 & 0.03 \\
\hline & 8 & $134 x, 4$ & not 0.13 & $\begin{array}{ll}u=0.13 \\
\end{array}$ & 0.14 & 0.14 & 0.14 & 0.14 & 0.14 & $5.59 \%$ & $-2.17 \%$ & $4.65 \%$ & $-0.72 \%$ & $1.84 \%$ & 0.002508 & 0.14 \\
\hline & 9 & unknown? & पि0.00 & 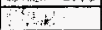 & 0.00 & 0.00 & 0.00 & 0.00 & & $\therefore$ & & & & & & \\
\hline & 0 & Total & 7100.00 & 100.00 & 100.00 & 100.00 & 100.00 & 100.00 & 100 & & & & & & 3.758291 & 100 \\
\hline
\end{tabular}




\begin{tabular}{|c|c|c|c|c|c|c|c|c|c|c|c|c|c|c|c|c|c|}
\hline 5 & Th $\mathbf{A}$ & AO & AP & $\overline{A Q}$ & $\overline{\mathbf{A R}}$ & A5 & AT & $\mathbf{A U}$ & AV & AW & $\overline{A X}$ & $\overline{A Y}$ & $A Z$ & BA & $\overline{B B}$ & $B C$ & $\overline{B D}$ \\
\hline 2 & $\div$ & Sample O & & & & & & & & Test Glass & & Test Glass & & Test Glass & & Test Glass & \\
\hline 83 & Oxide : : & $\therefore 1, \ldots$ & & $\mathrm{Tie}=\mathrm{SiO} 2$ & & Tie $=\mathrm{A}_{12} \mathrm{O} 3$ & & & & WHC3-SP1 & $M(1)$ & WHC3-SP1 & $M(1)$ & WHC3-SP1 & $M(1)$ & WHC3-SP1 & $M(1)$ \\
\hline $4:$ & Component & $A M(1)$ & & Table 3.5 & Theoretica & Table 3.5 & Theoretica & Avg loss & Avg loss & $19: 55$ & & 20:42 & & $21: 19$ & & $0: 42$ & \\
\hline 5 & $4+x+x_{1}^{2} \cdots$ & $3 \cdot 6$ & & $B$ feed & Testplan & B feed & Testplan & $\%$ of & $\%$ of glass & & & & & & & & \\
\hline 6 & $\neq \cdots$ & Al2O3 & avg & Target & Target & Target & Target & oxide & tot mass & & & & & & & & \\
\hline 7 & A1203 ? & 10.38 & 10.39 & $0.25 \%$ & $-2.20 \%$ & $0.00 \%$ & $0.00 \%$ & $-0.49 \%$ & -0.0494 & -0.00027 & & 0.017514 & & 0.00121 & & 0.001233 & \\
\hline B: & $8203 x$ & $7_{\therefore} \ldots 4.32$ & 4.32 & $14.91 \%$ & $15.08 \%$ & $14.70 \%$ & $16.91 \%$ & $15.40 \%$ & 0.770952 & 0.192675 & & $0.14 \overline{48}$ & & 0.219171 & & 0.14804 & \\
\hline 96 & $\mathrm{BaO}$ & $s_{s:}: 0.01$ & 0.01 & $-253.49 \%$ & NA & $-254.36 \%$ & NA & $-253.92 \%$ & -0.00762 & -2.35381 & & -2.3499 & & -2.49594 & & -2.53924 & \\
\hline 10 & Clita: & 0.01 & 0.01 & $97.21 \%$ & $96.93 \%$ & $97,20 \%$ & $96.99 \%$ & $97.08 \%$ & 0.351442 & 0.965579 & & 0.973553 & & 0.9862 & & 0.972059 & \\
\hline 11 & $\mathrm{CaO}$ & $\begin{array}{l}0.19 \\
\end{array}$ & 5.14 & $-1.48 \%$ & $-1.10 \%$ & $-1.74 \%$ & $1.07 \%$ & $-0.81 \%$ & -0.04054 & -0.01612 & & -0.01623 & & -0.0161 & & -0.0161 & \\
\hline 12 & $\overline{C 203: !}$ & 0.08 & 0.08 & $-68.71 \%$ & $-84.94 \%$ & $-69.13 \%$ & $-80.97 \%$ & $-75.94 \%$ & -0.03189 & -0.82935 & & -0.37041 & & -0.66851 & & -0.68918 & \\
\hline 13 & $\overline{C s 20}$ & 0.10 & 0.10 & $34.48 \%$ & $28.81 \%$ & $34.32 \%$ & $30.34 \%$ & $31.99 \%$ & 0.048142 & 0.365553 & & 0.385496 & & 0.452229 & & 0.344002 & \\
\hline 14 & $F \ldots$ & 0.00 & 0.00 & $99.32 \%$ & $99.27 \%$ & $99.32 \%$ & $99.28 \%$ & $99.30 \%$ & 0.29889 & 0.995796 & & 0.996799 & & 0.99332 & & 0.993237 & \\
\hline 16 & $\mathrm{FeO}$ & 0.84 & $0 . \overline{83}$ & $-1.44 \%$ & $-1.10 \%$ & $-1.70 \%$ & $1.07 \%$ & $-0.79 \%$ & -0.00641 & -0.01571 & & -0.01582 & & -0.0157 & & .0 .0157 & \\
\hline 16 & $\mathrm{Fe}_{203}$ & 0.00 & 0.00 & NA & NA & NA & NA & NA & na & & & & & & & & \\
\hline 17 & $\mathbf{K 2 O}$ & 1.34 & 1.34 & $22.48 \%$ & $16.65 \%$ & $.22 .29 \%$ & $18.44 \%$ & $19.97 \%$ & 0.328959 & 0.264515 & & 0.253619 & & \begin{tabular}{|l|}
0.270147 \\
\end{tabular} & & 0.223852 & \\
\hline 18 & $\mathrm{MgO}$ & 0.44 & $\overline{0.44}$ & $-73.92 \%$ & $-74.72 \%$ & $-74.35 \%$ & $-70.96 \%$ & $-73.49 \%$ & -0.18298 & -0.69032 & & -0.56775 & & -0.97171 & & -0.7413 & \\
\hline 19 & $\mathrm{MnO} 2$ & 0.02 & 0.02 & $-571.62 \%$ & $.903 .99 \%$ & $-573.29 \%$ & $-882.42 \%$ & $-732.83 \%$ & -0.01832 & -5.70762 & & -5.6998 & & -7.39024 & & -5.72455 & \\
\hline 22 & $\mathbf{P 2 0 5}$ & 0.10 & 0.10 & $56.81 \%$ & $53.38 \%$ & $56.71 \%$ & $54.38 \%$ & $55.32 \%$ & 0.117551 & 0.453678 & & 0.545262 & & 0.572895 & & 0.567605 & \\
\hline 23 & 503 & 0.01 & 0.01 & $95.60 \%$ & $95.42 \%$ & $95.59 \%$ & $95.52 \%$ & $95.53 \%$ & 0.225461 & 0.916503 & & 0.9166 & & 0.956482 & & 0.955943 & \\
\hline 24 & 5102 & 55.58 & 55.62 & $0.00 \%$ & $0.00 \%$ & $-0.25 \%$ & $2.15 \%$ & $0.48 \%$ & 0.26045 & 0.000271 & & -0.01815 & & -0.00121 & & -0.00124 & \\
\hline 25 & Sro & $u_{1}<0.11$ & 0.11 & $2.40 \%$ & $-5.68 \%$ & $2.16 \%$ & $-3.41 \%$ & NA & na & 0.073992 & & 0.075072 & & 0.034751 & & 0.022795 & \\
\hline 26 & $\mathrm{TiO} 2 \mathrm{~h}$ & 30.18 & 0.18 & $-87.79 \%$ & NA & $-88.25 \%$ & NA & $-88.02 \%$ & -0.0845 & -0.99133 & & -0.989 & & -1.29421 & & -0.88022 & \\
\hline 27 & $\mathrm{ZP2} \mathrm{AP}$ & -0.003 & 0.03 & $-536.28 \%$ & NA & $-537.85 \%$ & na & $-537.06 \%$ & -0.02685 & -7.04915 & & -5.02982 & & -7.39024 & & -5.37062 & \\
\hline 28 & Thoms & 30.14 & $+\infty \quad 0.14$ & $2.35 \%$ & $-5.68 \%$ & $2.10 \%$ & $-3.41 \%$ & $-1.16 \%$ & -0.00155 & 0.073479 & & 0.07456 & & 0.034216 & & 0.022254 & \\
\hline 29 & unknown? & $\because$ Pepe & & & & & & & & $\because$ & & & & & & & \\
\hline 30 & Total & $\therefore 100$ & 100 & & & & & -13.2473 & 1.599721 & & & & & & & & \\
\hline
\end{tabular}
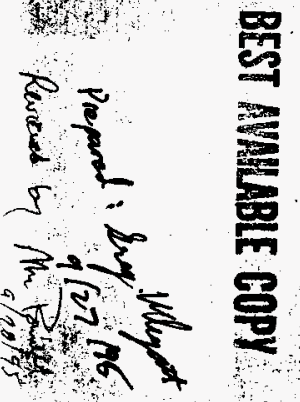


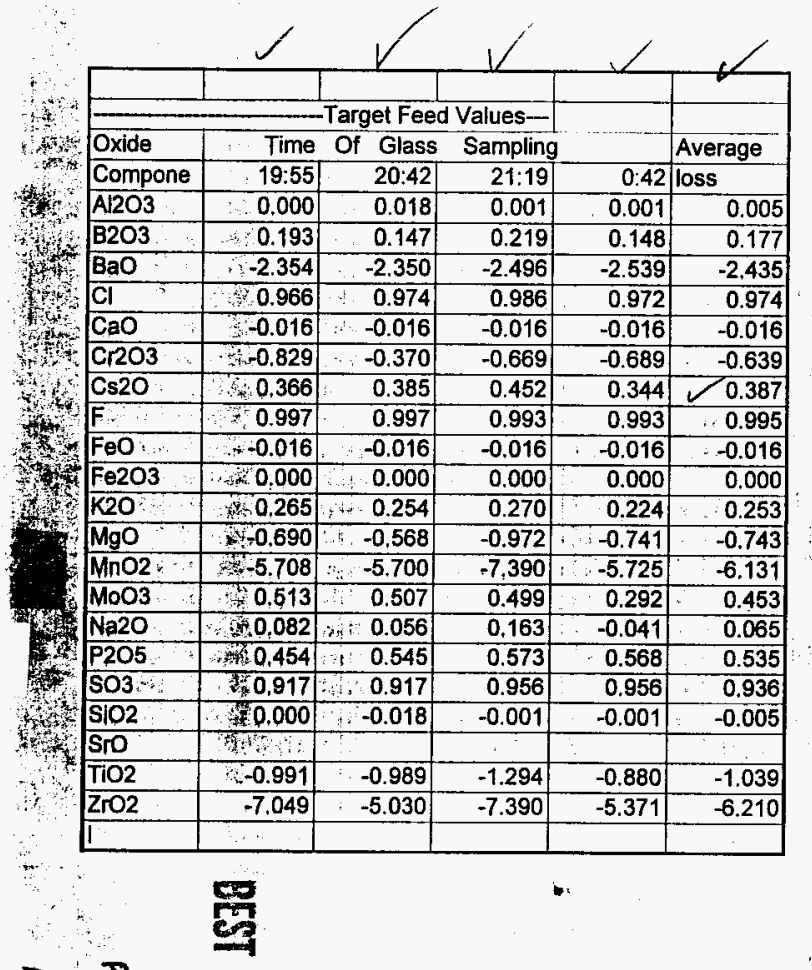

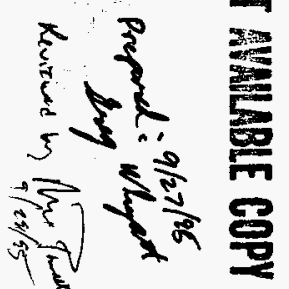


WHC-SD-WM-ER-517

Revision 0

APPENDIX E

REVIEW OF VECTRA TEST REPORT

E-1 
WHC-SD-WM-ER-517

Revision 0

This page intentionally left blank. 


\section{Vectra VE-SKULL Phase I LLW Melter Demonstration Entrainment and Volatility Estimates (MR Powell 9/8/95)}

(Revised 3/27/96 GA Whyatt)

The Vectra melter run analytical data were examined and estimates made of the relative volatility and entrainment for each oxide analyte. These analyses and the results are described below. Volatility estimates were made by comparing the melter feed and glass product compositions. Assuming uniform entrainment of feed and zero volatility for a known low volatility component, the fractional volatility of each glass oxide species can be estimated from feed and glass compositions. The entrainment rate of melter feed was estimated based on the observed average collection rate of low volatility oxides on isokinetically-gathered filter samples.

The phase I Vectra test included three "mass balance" periods, which are referred to as MT-2, MT-3, and MT-4. Mass balance test MT-2 used slurry feed and MT-3 and MT-4 used dried feed. Relatively little glass was produced by the feeds used for MT-3 and MT-4, so it is unlikely that the melter glass composition reached steady-state levels before the mass balance tests. For this reason, volatility estimates using tie component calculations were made only for MT-2. Also, slurry feeding is of greater interest since that is the recommended feeding approach. Aerosol sampling results are summarized for MT-3 and MT-4 at the end of this writeup.

\section{Entrainment}

Feed entrainment during the MT-2 mass balance period is estimated to be between $0.06 \%$ and $1.5 \%$. These estimates are derived from the aerosol sampling data included in the final Vectra report (WHC-SD-WM-VI-031, Rev 0.). The $0.06 \%$ to $1.5 \%$ range is established by the range of non-volatile species entrainment rates. Aerosol collection rates for aluminum, silicon, and iron were used in this calculation (see attached calculation worksheet). The average of the $\mathrm{Al}, \mathrm{Si}$, and $\mathrm{Fe}$ entrainment rates is $0.61 \%$. Two aerosol flow rate samples were taken during MT-2. The average bulk particulate flow rate as determined by these two samples was about $0.81 \mathrm{~kg} / \mathrm{h}$ total solids as oxides. If this solids flow rate was due exclusively to entrainment, then the entrainment rate would be $(0.81 / 33.6) * 100 \%=2.4 \%$ entrainment. Glass and feed composition data, however, indicate that some waste components were lost by volatilization rather than just entrainment. It is recommended that a $0.6 \%$ entrainment rate be taken as representative of the Vectra melter operated with slurry feed.

It should be noted that the aerosol data presented in the Vectra report gives widely varying entrainment estimates depending on which low-volatility analyte is selected for making the estimate. Using the MT-2 data, for example, the entrainment rate given by the second aerosol sample silica data is about $0.015 \%$. For the first aerosol sample alumina data, the entrainment rate is about $0.49 \%$. The calcium data for the first sample yields an entrainment 
rate of $14.2 \%$. Clearly, either the aerosol results are erroneous or the assumption of uniform entrainment of feed is incorrect. The description of the aerosol monitoring and analyses given in the Vectra report is not sufficient to determine whether the aerosol data are likely to be correct. Because of the large variability in entrainment estimates, the estimated $0.5 \%$ entrainment rate should be considered to be an "order of magnitude" entrainment estimate.

Feed entrainment during the MT-3 (dry simulated calcine feed material) was similar to MT-2 while entrainment during MT-4 (actual calcine feed material) may have been lower than during slurry feeding. The rates calculated for MT-3 based on non-volatile analytes of $\mathrm{Al}, \mathrm{Fe}$, Sr were $0.13,0.97,0.20$ respectively for an average of $0.43 \%$. The rates calculated for MT -4 based on non-volatile analytes of $\mathrm{Fe}$ and $\mathrm{Sr}$ were $0.056 \%$ and $0.084 \%$ respectively for an average of $0.07 \%$. Thus, the entrainment during MT-4 appears to have been lower than the slurry feeding or simulated dry calcine feeding periods.

\section{Volatility}

Fractional volatility estimates were generated for each of the glass oxide analytes. To make these calculations, it is necessary to select a tie-component, which is assumed to have zero volatility. Experience has shown that $\mathrm{SiO}_{2}, \mathrm{CaO}, \mathrm{Fe}_{2} \mathrm{O}_{3}$ and $\mathrm{Al}_{2} \mathrm{O}_{3}$ are generally good low volatility tie-components. The volatility calculations were made using the average MT-2 glass composition data and the estimated feed composition based on the feed makeup data records (see attached spreadsheet). An approach based on estimated feed composition was necessary because sampling and analysis of the feed was not adequate to characterize the melter feed. The variabilities of the glass analyses were used to estimate the $95 \%$ confidence intervals for each oxide concentration (uncertainty in the target feed composition was not quantified and was neglected). Systematic analytical errors are not included in these uncertainty estimates. The results are given in the attached spreadsheets and summarized in the tables below.

The attached spreadsheet gives volatility estimates for $\mathrm{MT}-2$ using $\mathrm{SiO}_{2}, \mathrm{Fe}_{2} \mathrm{O}_{3}, \mathrm{CaO}$, and $\mathrm{Al}_{2} \mathrm{O}_{3}$ as tie components. The feed and glass concentration data from mass balance run MT-2 were used to prepare these estimates. Too little feed was supplied to the melter during MT -3 and MT -4 to be sure that the glass composition had reached steady-state conditions. An average \% volatility was calculated for each analyte based on the average of the volatilities given by the four tie components. Along with the average \% volatility values are given estimated $95 \%$ confidence intervals. These confidence intervals were estimated based on the variance amongst the four fractional volatility estimates for each analyte. The fractional volatility data are presented in Table 1 . Table 1 does not include the minor components which were not in the original startup glass and may not have reached their full concentration prior to the mass balance period. The minor components not in the startup glass include $\mathrm{SrO}, \mathrm{Cs}_{2} \mathrm{O}$, $\mathrm{K}_{2} \mathrm{O}, \mathrm{F}, \mathrm{Cl}, \mathrm{I}, \mathrm{SO}_{3}$ and $\mathrm{P}_{2} \mathrm{O}_{5}$. Because these minor components were not at steady state, their volatilities were calculated using $\mathrm{SrO}$ as the tie component. These results are provided in Table 2. If it is assumed that the volatility occurs from the cold cap prior to incorporation 
into the molten glass, then this approach should provide accurate volatility estimates.

Table 1. Volatilities based on $\mathrm{SiO}_{2}, \mathrm{Al}_{2} \mathrm{O}_{3}, \mathrm{CaO}, \mathrm{Fe}_{2} \mathrm{O}_{3}$ tie components ${ }^{(a)}$

\begin{tabular}{|c|c|c|c|c|c|}
\hline \multicolumn{6}{|c|}{ Estimate of Waste Oxide Volatilities } \\
\hline Oxide & $\mathrm{SiO}_{2} \mathrm{Tie}(\%)$ & $\begin{array}{c}\mathrm{Al}_{2} \mathrm{O}_{3} \mathrm{Tie} \\
(\%)\end{array}$ & $\begin{array}{c}\mathrm{CaO} \text { Tie } \\
(\%)\end{array}$ & $\begin{array}{c}\mathrm{Fe}_{2} \mathrm{O}_{3} \\
(\%)\end{array}$ & Avg. $(\%)^{(\mathrm{b})}$ \\
\hline $\mathrm{Al}_{2} \mathrm{O}_{3}$ & $8.59 \pm 5.0$ & 0 & $1.4 \pm 5.3$ & $3.1 \pm 6.1$ & $3.4 \pm 3.0$ \\
\hline $\mathrm{B}_{2} \mathrm{O}_{3}$ & $19.4 \pm 3.4$ & $11.4 \pm 5.6$ & $12.6 \pm 3.5$ & $14.2 \pm 4.4$ & $14.4 \pm 2.2$ \\
\hline $\mathrm{BaO}$ & No Data & No Data & No Data & No Data & No Data \\
\hline $\mathrm{CaO}$ & $7.71 \pm 2.4$ & $-1.4 \pm 5.5$ & 0 & $1.8 \pm 3.9$ & $2.0 \pm 1.9$ \\
\hline $\mathrm{Cr}_{2} \mathrm{O}_{3}$ & $-638 \pm 27$ & $-710 \pm 49$ & $-699 \pm 27$ & $-685 \pm 37$ & $-683 \pm 18$ \\
\hline $\mathrm{Fe}_{2} \mathrm{O}_{3}$ & $6.1 \pm 3.9$ & $-3.2 \pm 6.5$ & $-1.8 \pm 4.0$ & 0 & $0.3 \pm 2.5$ \\
\hline $\mathrm{Li}_{2} \mathrm{O}$ & No Data & No Data & No Data & No Data & No Data \\
\hline $\mathrm{MgO}$ & $3.6 \pm 3.7$ & $-5.9 \pm 6.5$ & $-4.5 \pm 3.8$ & $-2.6 \pm 5.0$ & $-2.3 \pm 2.4$ \\
\hline $\mathrm{MnO}_{2}$ & $-525 \pm 272$ & $-586 \pm 301$ & $-577 \pm 295$ & $-565 \pm 290$ & $-563 \pm 145$ \\
\hline $\mathrm{MoO}_{3}$ & $-40.5 \pm 11.0$ & $-54.3 \pm 14.1$ & $-52.2 \pm 11.8$ & $-49.5 \pm 13$ & $-49 \pm 6.2$ \\
\hline $\mathrm{Na}_{2} \mathrm{O}$ & $17.7 \pm 2.3$ & $-9.6 \pm 5.0$ & $10.8 \pm 2.3$ & $12.4 \pm 3.6$ & $12.6 \pm 1.7$ \\
\hline $\mathrm{NiO}$ & No Data & No Data & No Data & No Data & No Data \\
\hline $\mathrm{SiO}_{2}$ & 0 & $-9.8 \pm 6.1$ & $-8.4 \pm 2.8$ & $-6.4 \pm 4.4$ & $-6.2 \pm 2.1$ \\
\hline $\mathrm{TiO}_{2}$ & No Data & No Data & No Data & No Data & No Data \\
\hline $\mathrm{ZrO}_{2}$ & No Data & No Data & No Data & No Data & No Data \\
\hline
\end{tabular}

(a) The minor components $\mathrm{SrO}, \mathrm{Cs}_{2} \mathrm{O}, \mathrm{K}_{2} \mathrm{O}, \mathrm{F}, \mathrm{Cl}, \mathrm{I}, \mathrm{SO}_{3}$ and $\mathrm{P}_{2} \mathrm{O}_{5}$ are provided in Table 2.

(b) The confidence intervals on the average do not account for cross correlation of the different tie component results and may therefore underestimate the magnitude of the potential error.

(c) Statistics are not presented for $\mathrm{SO}_{3}$ because only two analytical results above detection limits were obtained. 
Table 2. Minor Component Volatile Loss Based on SrO Tie Component

\begin{tabular}{|l|l|}
\hline Component & Percent Volatile Loss \\
\hline $\mathrm{Cl}$ & 64 \\
\hline $\mathrm{Cs}_{2} \mathrm{O}$ & 41 \\
\hline $\mathrm{F}$ & 9 \\
\hline $\mathrm{K}_{2} \mathrm{O}$ & 15 \\
\hline $\mathrm{I}$ & 83 \\
\hline $\mathrm{P}_{2} \mathrm{O}_{5}$ & -45 \\
\hline $\mathrm{SO}_{3}$ & 85 \\
\hline
\end{tabular}

However, this approach could potentially underestimate the volatility if the volatility has a significant dependence on the concentration of the volatile species within the molten glass. In any case, the result should be more accurate than comparing to major glass components that are at steady state concentrations which results in a significant over estimation of volatility. Taking this approach does result in making all tie-component results dependant on the $\mathrm{Sr}$ analysis results.

The tie component volatility analysis indicates that during the MT-2 mass balance run significant volatility was observed for $\mathrm{B}_{2} \mathrm{O}_{3}, \mathrm{Cs}_{2} \mathrm{O}, \mathrm{K}_{2} \mathrm{O}, \mathrm{Na}_{2} \mathrm{O}, \mathrm{SO}_{3}, \mathrm{Cl}$, and $\mathrm{I}$.

The tie component results in Table 1 determined negative volatilities for $\mathrm{Cr}_{2} \mathrm{O}_{3}, \mathrm{MnO}_{2}$, and $\mathrm{MoO}_{3}$. The negative $\mathrm{Cr}_{2} \mathrm{O}_{3}$ volatility is probably due to refractory and electrode sheath degradation during the run. The Vectra report notes that the elevated chrome concentrations in the glass samples are consistent with the amount of observed refractory loss (p. 3-9 of final report). The negative $\mathrm{MnO}_{2}$ volatility is probably just an artifact of the low $\mathrm{MnO}_{2}$ concentrations in the feed and the glass. The $\mathrm{MnO}_{2}$ concentrations reported in the glass samples are all at or near the $\mathrm{MnO}_{2}$ detection limit. Negative $\mathrm{MoO}_{3}$ volatilities are expected as a consequence of the erosion of the molybdenum electrodes in the melter. During the 28 days of total melter operation, more than $140 \mathrm{~kg}$ of electrode mass was lost. This loss rate is more than large enough to account for the elevated molybdenum oxide concentrations observed during the MT-2 mass balance run.

The results in Table 2 determined a significant negative volatility for $\mathrm{P}_{2} \mathrm{O}_{5}$. No source has been identified and the single feed analysis raises suspicions that the feed may have been too high in $\mathrm{P}_{2} \mathrm{O}_{5}$ by roughly a factor of 2 . 


\section{Volatility Comparisons to Aerosol Sampling Data}

A second method to determine volatilities is to examine the method 5 data, accounting for some amount of uniform entrainment of feed ( $0.6 \mathrm{wt} \%$ in this case) and compare the aerosol oxide emission rates with the feed rates to the melter. Two 1 hour aerosol samples were obtained during the MT-2 slurry feeding period. A comparison of the volatility results from aerosol samples to those using the tie component method are shown in Table 3 . The aerosol samples provide volatility estimates that are systematically lower than the tie component method results by a factor of 1.5 to 3.5 (excluding $F$ which typically has larger analytical uncertainty).

Table 3. Comparison of Aerosol and Tie Component Based Volatilities

\begin{tabular}{|l|l|l|l|}
\hline Oxide & Aerosol Sample Volatility & Tie Component Volatility & Factor Difference \\
\hline $\mathrm{B}_{2} \mathrm{O}_{3}$ & 6.8 & 14 & 2.1 \\
\hline $\mathrm{Cs}_{2} \mathrm{O}$ & no data & 41 & NA \\
\hline $\mathrm{Cl}$ & 42 & 64 & 1.5 \\
\hline $\mathrm{F}$ & 15 & 9 & 0.6 \\
\hline $\mathrm{I}$ & 32 & 83 & 2.6 \\
\hline $\mathrm{K}_{2} \mathrm{O}$ & 8.5 & 15 & 1.8 \\
\hline $\mathrm{Na}_{2} \mathrm{O}$ & 3.6 & 13 & 3.6 \\
\hline $\mathrm{P}_{2} \mathrm{O}_{5}$ & 1.1 & -45 & \\
\hline $\mathrm{SO}_{3}$ & 56 & 85 & 1.5 \\
\hline
\end{tabular}

It is expected that the reason for the generally lower loses predicted by the aerosol samples is that there was loss of particulate between the melter exit and the sample point. Sampling was performed on a section of the offgas line outside the building some distance from the melter exit. Deposition in the line between the melter and the sample point cannot be ruled out and in fact some loss is expected. Other possible sources of the differences include:

-Unsteady melter operation such that higher volatility occurred during periods when aerosol sampling was not being performed.

-Detection limits for analysis of scrub solutions was too high to achieve detection of gaseous emissions of acid gases. Thus, offgas sampling results only include the aerosol portion of emissions.

-Variations in offgas flow during operation could account for errors in method 5 
sampling.

In addition, however, errors in feed makeup such that the feed is deficient in the volatile components could account for some of the differences as well. The tie component calculation based on feed targets would then include deficiencies in the volatility result. There is only one slurry feed sample analysis available during the MT-2 processing (V1M2F35108PA). The sample number indicates it is a mixed feed sample taken during MT-2 from the feed slurry tank and analyzed at PNL. The analytical results report very low silica content $\left(\mathrm{SiO}_{2}: \mathrm{Na}_{2} \mathrm{O}\right.$ wt ratio of 0.5 vs 3.1 target) which indicates the sample may not be representative. The ratio of $\mathrm{Na}_{2} \mathrm{O}$ to $\mathrm{CaO}$ for the feed analysis (5.97) and target values (5.89) indicates good agreement between sodium and calcium levels. Similarly, the $\mathrm{Na}_{2} \mathrm{O}$ to $\mathrm{MgO}$ ratios agree to within $3 \%$. However, the aluminum to sodium ratios are feed analysis 1.49 and target 1.80 indicating either a $17 \%$ deficiency in sodium or excess aluminum. The feed analysis does perhaps explain the results for $\mathrm{P}_{2} \mathrm{O}_{5}$ suggesting roughly a factor of 2 excess in the feed. While there is insufficient data to reach a firm conclusion on possible feed deficiencies, it is expected that large measurement errors in feed makeup for major components such as sodium or boron are unlikely.

Conclusion: The aerosol samples are believed to be the better source of volatility data for $F$ due to the analytical difficulties for the glass samples for this analyte. The volatile loss for $\mathrm{P}_{2} \mathrm{O}_{5}$ is also best determined by aerosol measurement. In this case there is uncertainty about the actual feed concentration and the aerosol result is less sensitive to this type of error. For other analytes, the tie component calculation with the appropriate tie component is believed to be the best data.

\section{Overall Mass Balance for MT-2}

At first glance, the entrainment and volatility estimates are not consistent with the overall mass balance data. During mass balance test MT-2, a total of $865 \mathrm{~kg}$ of slurry feed was delivered to the melter. The theoretical glass mass produced by $865 \mathrm{~kg}$ of slurry feed is about $469 \mathrm{~kg}$. Only $406 \mathrm{~kg}$ of glass was withdrawn from the melter during MT-2. Thus, there is about $63 \mathrm{~kg}$ of missing glass mass. The aerosol flow rate data account for roughly $9 \mathrm{~kg}$ of this mass, leaving $54 \mathrm{~kg}$ of glass unaccounted for. Despite the discrepancy, the Vectra report does not address where this mass went. It is expected that due to the intermittent pouring from the Vectra melter, there was some difference between starting and ending levels within the melter. Although the melter was on load cells during the run, no data from the load cells is reported in the final report. Assuming a specific gravity of 2.6 for the glass and a inside diameter of the melter of $3.2 \mathrm{ft}$, the change in level to account for the missing $54 \mathrm{~kg}$ is only 2.8 $\mathrm{cm}$. Some mass may also have collected in the offgas system prior to the aerosol sample point.

\section{MT-3 Aerosol Results}

During MT-3, a simulated calcine material was fed which consisted of dry materials with an 
average composition matching the expected composition of calcined feed material. No feed characterization information was available so any comparisons to the feed rate are made to the target feed compositions. The feed rate was highly variable such that there is significant uncertainty about the appropriate feed rate to use for comparison of aerosol emission rates. The aerosol samples indicate an entrainment rate of $0.43 \%$ (average of $\mathrm{Al}, \mathrm{Fe}, \mathrm{Sr}$ entrainment values). The volatile losses of selected components are shown below. A spreadsheet with complete results is attached. Due to poor detection limits, the results shown here include only particulate losses and acid gases would be in addition to the losses described here.

MT-3 Aerosol Sample Based Volatile Losses

\begin{tabular}{|l|l|l|}
\hline Component & Total Aerosol Loss (\%) & $\begin{array}{l}\text { Volatility (assuming 0.43\% } \\
\text { entrainment) }\end{array}$ \\
\hline $\mathrm{B}_{2} \mathrm{O}$ & 3.3 & 2.9 \\
\hline $\mathrm{MoO}_{3}$ & 3.81 & 3.4 \\
\hline $\mathrm{Na}_{2} \mathrm{O}$ & 0.90 & 0.5 \\
\hline $\mathrm{SO}_{3}$ & 6.84 & 6.4 \\
\hline $\mathrm{Cl}$ & 21.6 & 21.2 \\
\hline $\mathrm{F}$ & 2.1 & 1.7 \\
\hline
\end{tabular}

\section{MT-4 Aerosol Results}

During MT -4 , a calcined feed material was processed through the melter. Due to caking problems in the calciner, boric acid was added after the calcining operation. No feed characterization information was available so any comparisons to the feed rate are made to the target feed compositions. The feed rate was highly variable such that there is significant uncertainty about the appropriate feed rate to use for comparison of aerosol emission rates. The aerosol samples indicate an entrainment rate of $0.07 \%$ (average of $\mathrm{Fe}$ and $\mathrm{Sr}$ entrainment values). The volatile losses of selected components are shown below. A spreadsheet with complete results is attached. Volatile losses for the actual calcine were somewhat higher than the simulated calcine. Significant sources of sulfur and chloride are indicated because the loss of these components exceed the feed by factors of 3.8 and 3.0 based on particulate emissions alone.

Due to poor detection limits, the results shown here include only particulate losses and acid gases would be in addition to the losses described here. Losses of sulfur were high enough to be detected in scrub solutions during this processing but aerosol measurements alone indicate 
that the losses were far in excess of the feed amount so the $\mathrm{SO}_{2}$ emissions were not inlcluded in the calculations. The source of excess sulfur is not known.

MT-4 Aerosol Sample Based Volatile Losses

\begin{tabular}{|l|l|l|}
\hline Component & Total Aerosol Loss (\%) & $\begin{array}{l}\text { Volatility (assuming 0.07\% } \\
\text { entrainment) }\end{array}$ \\
\hline $\mathrm{B}_{2} \mathrm{O}$ & 21.4 & 21.3 \\
\hline $\mathrm{MoO}$ & 19.5 & 19.4 \\
\hline $\mathrm{Na}_{2} \mathrm{O}$ & 10.6 & 10.5 \\
\hline $\mathrm{SO}_{3}$ & 381 & 381 \\
\hline $\mathrm{Cl}$ & 297 & 297 \\
\hline $\mathrm{F}$ & 11.2 & 11.1 \\
\hline
\end{tabular}

\section{Fluid Bed Calciner/RVR Calciner Results}

A fluid bed calciner and rotary dryer were tested for preparation of calcined feed. Discussion of the experiments is provided in the Vectra final report. In general, the rotary dryer suffered from severe caking such that it is not recommended for drying the feed. The fluid bed calciner was most successful operating at about $500^{\circ} \mathrm{C}$ and with $100 \%$ stoichiometric sucrose addition. The mass balance provided indicates about $91 \%$ of the $\mathrm{NO}_{x}$ is converted to $\mathrm{N}_{2}$ with $3.6 \%$ converted to $\mathrm{NO}_{x}, 4.7 \%$ converted to $\mathrm{N}_{2} \mathrm{O}$, and $0.4 \%$ converted to ammonia. Insufficient information is provided in the report to verify the numbers. The sample was apparently taken downstream from a caustic scrubber which adds uncertainty to the result. The results indicate much lower ammonia production and much higher conversion to nitrogen than the USBM tests in which material was heated in a laboratory setting to evaluate the gases generated (USBM used sucrose addition and a belt dryer). 
Vectra Mt2

3/27/96 5:28 PM

\begin{tabular}{|c|c|c|c|c|c|c|c|c|c|}
\hline \multicolumn{3}{|c|}{ Greg A Whyatt 3/27/96 } & & & & & & & \\
\hline \multicolumn{10}{|c|}{ Vectra Slurry Feed Method 5 Loss Estimates } \\
\hline \multicolumn{10}{|c|}{ Mass balance period MT2 } \\
\hline \multicolumn{3}{|c|}{ Total Glass Mass over 14 hours } & 406.42 & $\mathrm{~kg}$ & & & & & \\
\hline \multirow{4}{*}{-} & & & & & average & & & & Volatility \\
\hline & & & & & offgas & Feed & & Percent & (adjust \\
\hline & & & & & mass & Oxides & Feed & Partition & assuming \\
\hline & & & avg & oxide & oxide & MT-2 & Oxides & oxide & $0.6 \mathrm{wt} \%$ \\
\hline species & $\mathrm{kg} / \mathrm{h} \# 1$ & $\mathrm{~kg} / \mathrm{h} \# 2$ & $\mathrm{~kg} / \mathrm{h}$ & form & $\mathrm{kg} / \mathrm{hr}$ & $\mathrm{kg}$ & $\mathrm{kg} / \mathrm{hr}$ & to offgas & entrainment) \\
\hline Al & 0.00857 & 0.00213 & 0.00535 & $\mathrm{Al} 2 \mathrm{O} 3$ & 0.010109 & 46.49655 & 3.321182 & 0.304382 & -0.295618 \\
\hline $\mathrm{B}$ & 0.08026 & 0.04728 & 0.06377 & $\mathrm{~B} 2 \mathrm{O} 3$ & 0.205337 & 39.10279 & 2.793056 & 7.351693 & 6.75169259 \\
\hline $\mathrm{CaCO} 3$ & 0.25623 & 0.16532 & 0.210775 & $\mathrm{CaO}$ & 0.118108 & 14,1717 & 1.012264 & 11.66772 & 11.0677152 \\
\hline $\mathrm{Cl}$ & 0.04776 & 0.04457 & 0.046165 & $\mathrm{Cl}$ & 0.046165 & 1.52798 & $0.109 \overline{141}$ & 42.29833 & 41.6983263 \\
\hline $\mathrm{Cr}$ & 0.00078 & 0.0003 & 0.00054 & $\mathrm{Cr} 2 \mathrm{O} 3$ & 0.000789 & 0.177428 & 0.012673 & 6.227584 & 5.62758431 \\
\hline$F$ & 0.01505 & 0.01321 & 0.01413 & $\mathbf{F}$ & 0.01413 & 1.279603 & 0.0914 & 15.45949 & 14.8594884 \\
\hline $\mathrm{Fe}$ & 0.00531 & 0.0019 & 0.003605 & $\mathrm{Fe} 2 \mathrm{O} 3$ & 0.005154 & 4,88542 & 0.348959 & 1.477033 & 0.87703282 \\
\hline 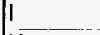 & 0.01711 & 0.0095 & 0.013305 & & 0.013305 & 0.569781 & 0.040699 & 3269148 & 32.0914848 \\
\hline K & 0.04123 & 0.02689 & 0.03406 & $\mathrm{~K} 2 \mathrm{O}$ & 0.041028 & 6.344135 & 0.453152 & 9.054004 & 8.45400384 \\
\hline $\mathrm{Mn}$ & 0.00013 & 000004 & 0.000085 & $\mathrm{MnO} 2$ & $0.000 \overline{135}$ & 0.009757 & $0.00069 \overline{7}$ & 19.29985 & 18.6998461 \\
\hline Mo & 0.00408 & 0.06714 & 0.03561 & $\mathrm{MoO3}$ & 0.053426 & 0.646267 & 0.046162 & 115.7364 & 115.136428 \\
\hline $\mathrm{Na}$ & 0.22073 & 0.14928 & 0.185005 & $\mathrm{Na2O}$ & $0 . \overline{249383}$ & 83.5109 & 5.965064 & 4.180719 & 3.58071866 \\
\hline$P$ & 0.00028 & 0,00058 & 0.00043 & $\mathrm{P} 205$ & 0.000985 & 0.82856 & 0.059183 & 1.664854 & 1.06485352 \\
\hline $\mathrm{SO} 4$ & 0.05368 & 0.03721 & 0.045445 & 503 & 0.037876 & 0.934643 & 0.06676 & 56.7342 & 56.1341957 \\
\hline $\mathrm{Si}$ & 0.00892 & 0,00129 & 0.005105 & $\mathrm{SiO} 2$ & 0.010921 & 258.0784 & 18.43417 & 0.059246 & -0.5407545 \\
\hline \multirow[t]{2}{*}{ Sr } & 0.000062 & 0.000011 & $3.65 \mathrm{E}-05$ & SrO & $4.32 \mathrm{E}-05$ & 0.465237 & 0.033231 & 0.129894 & -0.4701065 \\
\hline & & & & & other & 10.9 & & & \\
\hline totals & 0.760182 & 0.566651 & 0.663417 & totals & 0.806895 & 469.9292 & 32.7878 & & \\
\hline
\end{tabular}

Reviowed 
VSIM (MT-3)

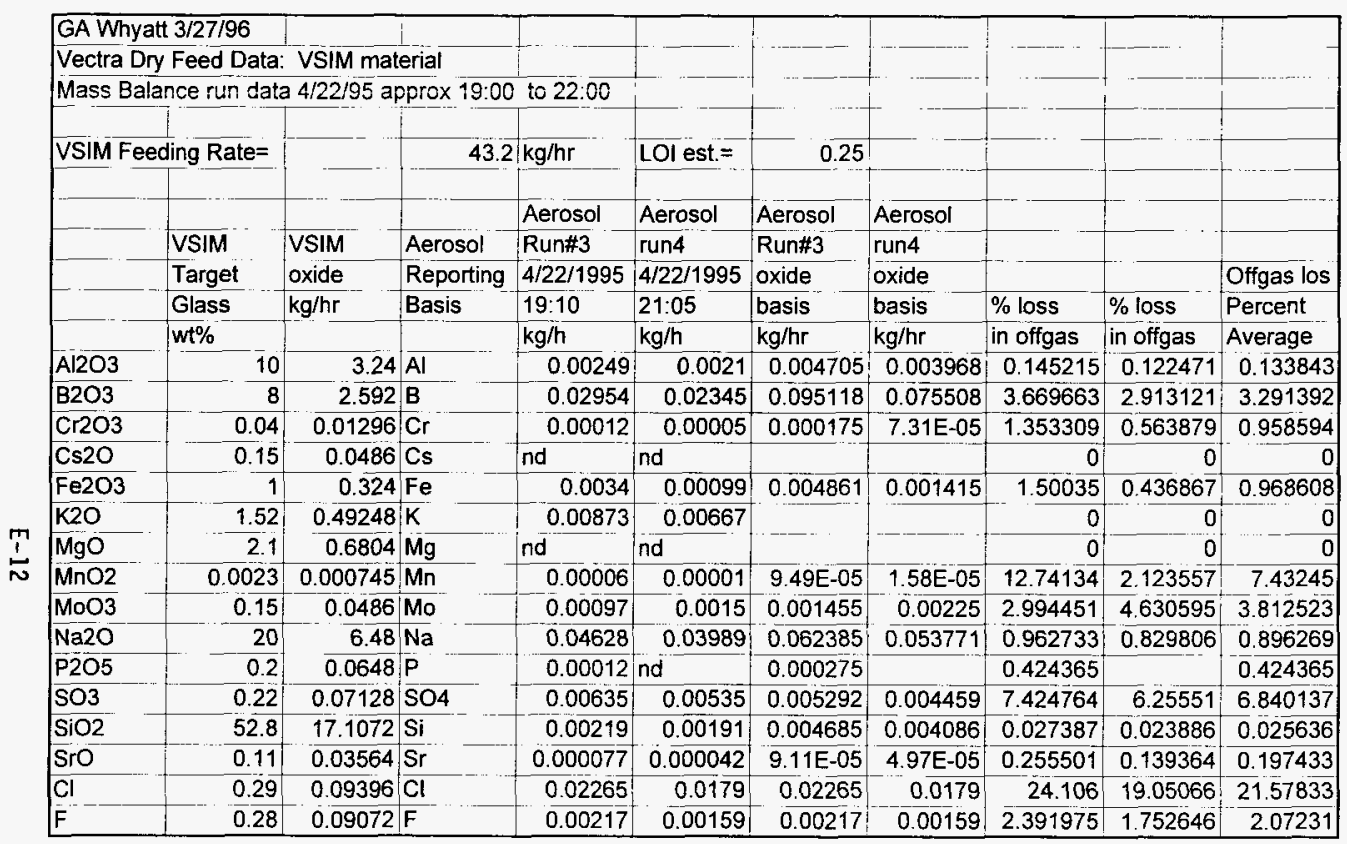

Rewetert bi 


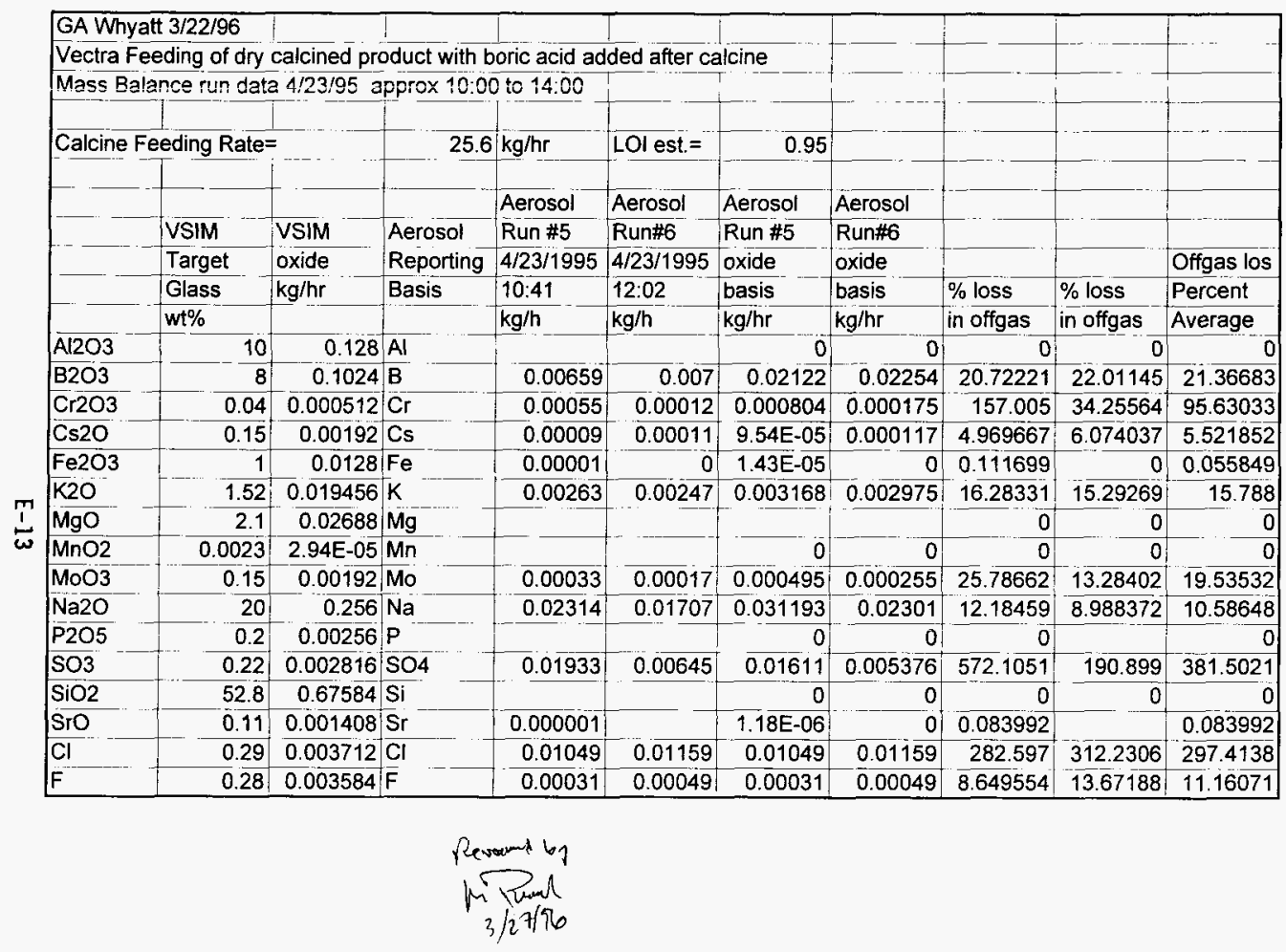


Vectra MT-2

Minor Component

Vólatility Estimate

Based on SrO Tie Calculation

3/17/96 3:20 PM

\begin{tabular}{|c|c|c|c|c|c|c|c|c|c|c|c|c|}
\hline \multirow{2}{*}{\multicolumn{13}{|c|}{\begin{tabular}{|l|} 
Correction of Fractional Volatility Estimates for Vectra MT-2 \\
Mass Balance Test. (GA Whyatt $3 / 17 / 96$ )
\end{tabular}}} \\
\hline & & & & & & & & & & & & \\
\hline & & & & & & & & & & & & \\
\hline & & & & & & & & & & & & $\mathrm{pg} 3-78$ \\
\hline \multirow[t]{3}{*}{ Target } & \multicolumn{2}{|c|}{ Feed Compositions } & \multicolumn{3}{|c|}{ (from feed makeup data, p. 3-76) } & & & & & & & \multirow{2}{*}{$\begin{array}{l}\mathrm{Na} 2 \mathrm{O} \\
\text { target } \mathrm{wt} \%\end{array}$} \\
\hline & & & & & & & & & & & & \\
\hline & SrO & $\mathrm{Cl}$ & $\mathrm{Cs} 2 \mathrm{O}$ & Cs2O & $\mathrm{K} 2 \mathrm{O}$ & $\mathrm{K} 2 \mathrm{O}$ & 1 & $F$ & P205 & $\mathrm{SO} 3$ & $\mathrm{Na2O}$ & in glass \\
\hline g/l simulant & 1.727 & 5.672 & 2.348 & 2.348 & 23.55 & 23.55 & 2.115 & 4.75 & 3.076 & 3.4695 & 310 & 17.17 \\
\hline wt \% glass & 0.095654 & 0.314156 & 0.130049 & 0.130049 & 1.304366 & 1.304366 & 0.117144 & $4 \quad 0.263089$ & 0.170371 & 0.192166 & 17.17 & \\
\hline & & & & & & & & & & & & \\
\hline & Glass Com & positions & & & & & & & & & & \\
\hline & & & USGS/C & PNL & USGS/C & PNL & & & & & & \\
\hline Sample \# & Sro & $\mathrm{Cl}$ & Cs2O & $\mathrm{Cs} 2 \mathrm{O}$ & $\mathrm{K} 2 \mathrm{O}$ & $K 20$ & $\mathrm{~T}$ & $F$ & $\mathrm{P} 205$ & $\mathrm{SO} 3$ & & \\
\hline & 0.0503 & 0.05 & 0.035 & 0.078 & $0.5 \overline{8}$ & 1.16 & 00093 & 0125 & 0.12 & 0.006 & & \\
\hline 2 & $0 . \overline{05} \overline{5}$ & 0.07 & 0.048 & & 0.63 & & 0.0118 & $\begin{array}{r}0.125 \\
0.14\end{array}$ & $\frac{0.12}{0.16}$ & & & \\
\hline 3 & 0.055 & 0.07 & 0.05 & & 0.67 & & 0.0131 & 0.14 & 0.16 & & & \\
\hline & 0.0597 & 0.07 & 0.044 & $0 . \overline{112}$ & 0.68 & 2.22 & 0.0123 & 0.145 & 0.126 & 0.029 & & \\
\hline & & & & & & & & & & & & \\
\hline Percent Volatil & ile Loss Calo & culations & & & & & $---\cdot$ & & & & & \\
\hline & & & & & & & & & & & & \\
\hline & & & USGSIC & PNL & USGS/C & PNL & & & & & & \\
\hline Sample \# & Sro & $\mathrm{Cl}$ & $\mathrm{Cs} 20$ & $\mathrm{Cs2O}$ & $\mathrm{K} 2 \mathrm{O}$ & K & 1 & $F$ & $\mathrm{P} 2 \mathrm{O} 5$ & $\mathrm{SO} 3$ & & \\
\hline & & & & & & & & & & & & \\
\hline 1. & & 69.73378 & 48.8207 & -14.0567 & 15.44069 & -69.1186 & 84.90278 & 9.647379 & -33.9428 & 94.06243 & & \\
\hline 2 & & 61.24824 & 35.8092 & & 16 & & 82.48132 & 7.452632 & -63.329 & & & \\
\hline 3. & & 61.24824 & 33.13458 & & 10.66667 & & 80.5513 & $7 . \overline{452632}$ & $-63.3 \overline{29}$ & & & \\
\hline 4 & & 64.29905 & 45.79085 & -37.9869 & 16.47125 & -172.697 & 83.17664 & 11.69356 & -18.4956 & 75.8204 & & \\
\hline Avg $\%$ volatile & loss & 64.13233 & 40.88883 & -26.0218 & 14.64465 & -120.908 & $82.7 ; 801$ & 9.061549 & -44.7741 & 84.94142 & & \\
\hline & & & & & & & & & & & & \\
\hline$\# 1$ in & & & $1 \mathrm{M} 2 / \mathrm{G}$ & 31 & U1, & & & & & & & \\
\hline plear Inclu & udes s & ea & f $V 1 M 2 / G 3$ & $2-0$ & & & & & & & & \\
\hline $\begin{array}{l}\text { sample\#3 inclu } \\
\text { sample\#4 inclu }\end{array}$ & $\begin{array}{l}\text { udes sampl } \\
\text { udes sampl }\end{array}$ & $\begin{array}{l}\text { eanalysis } \\
\text { e analysis }\end{array}$ & $\mathrm{V} / \mathrm{M} 2 / \mathrm{G}$ & $\frac{2-0380}{20408 \pi}$ & +7 & & & & & & & \\
\hline
\end{tabular}

Revrent in

Page 1 M $3 / 27 / 96$ 


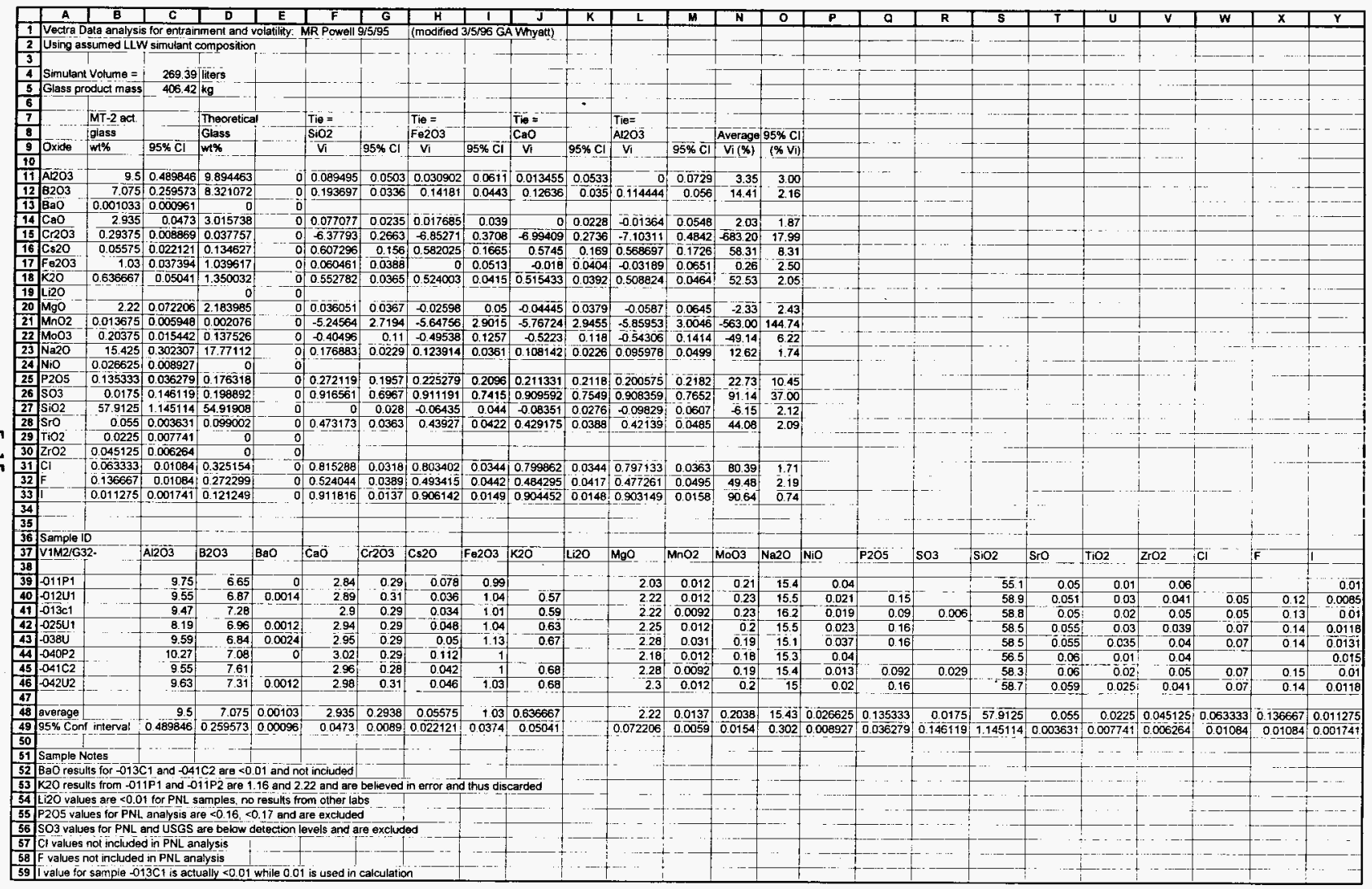


Vectra MT2

3/14/96 2:19 PM

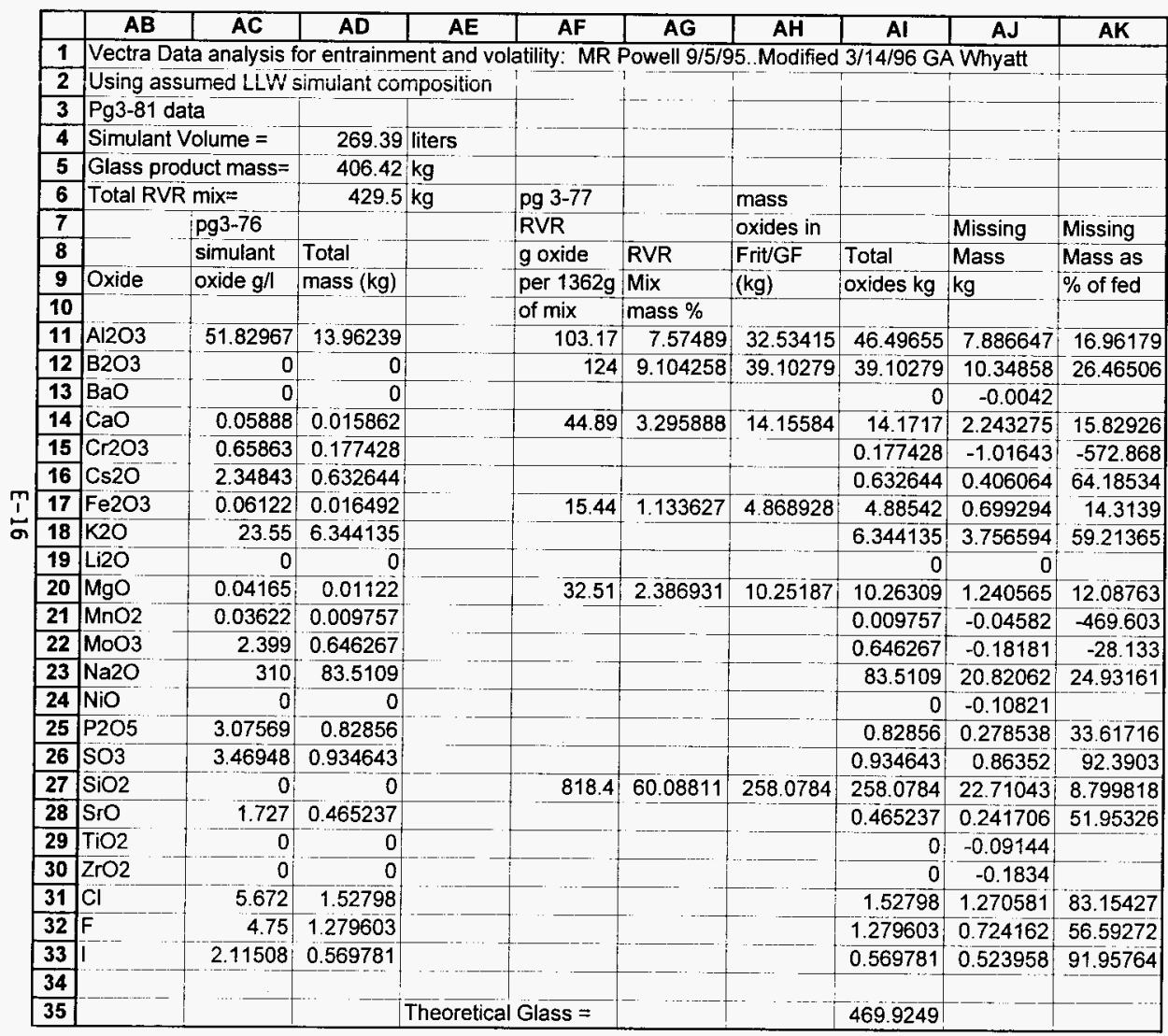




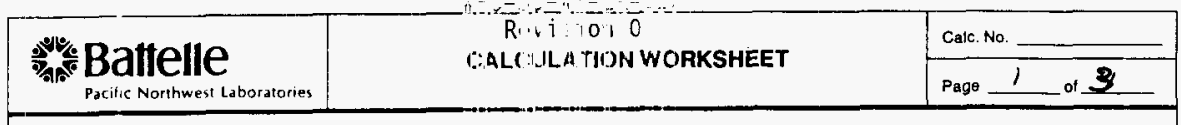

Revised volatility estimates fir Vesta MT -2 mass balance run. The glass composition in the melted during the MT.2 mass balance
was datable for the major aside iomponeds. However, some of the melter before the mit ins. present in the feed delivered to the

Ceases of this, fractional volatilities calculated for certain minor components will be biased' high if a major component oxide is used as the tie cornperent (ie,, if $\mathrm{Al}_{2} \mathrm{O}_{3}, \mathrm{CnO}_{1}$ or $\mathrm{SiO}_{2}$ arr used as vie component). Improved volatility estimates can be obtained by using a zero -volatility (with $V_{1}=0$ ) selected must have not been present in the itart-uf feed, ECO rests the or criteria.

Calculation of revised fractional volatilities for the minor components not present in the start up feed $\left(\mathrm{Cl}, \mathrm{Cs}_{\mathrm{s}}\right.$, and I)

Fractional volatility is calculated by:

$$
V_{i}=1-\frac{c_{g i} \cdot C_{f, t i c}}{c_{f i} \cdot c_{g} \text {, tic }}
$$

BEST MUMBLE COPY

whore

$$
C_{f i}=\text { Concentration of component }
$$

$C_{S i}=$ Concentration of Component $i$ in the glass

$C_{f}$, tic $=$ Concentration of tie component in the feed

$\left(y^{5}\right)+i=$ Concentration of tie component in the glass

The relevant concentration data are:

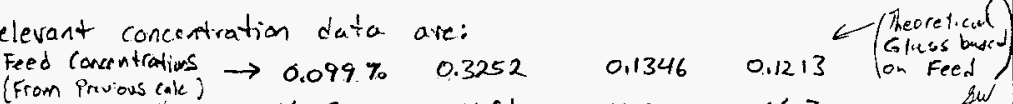

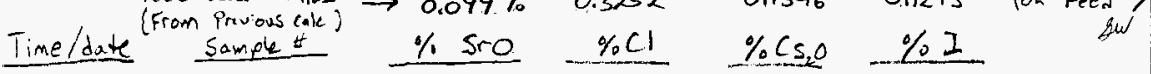

$4 / 21 \quad 22: 30$

22.30

$v 1 m 2 / 632-a 1 p 1 \quad .05$

$.078^{*}, \quad .01$

22.30

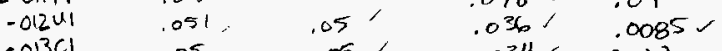

A. average concentrations $\rightarrow \frac{.05}{.0503} \frac{.05}{.05} \frac{.034}{.035} \frac{(.01)<\text { et. limit nat. }}{.0093}$ used.

$4 / 22 \quad 8: 00$

$v 1+2 / 632-025 d$

.055

$.048, .0118 \mathrm{r}$

$4 / 22 \quad 11: 20$

$v 1 m 2 / 6 \geq 2-0384$

.055

.07

$.050 \%, 0131 \mathrm{r}$

4/22 14:00

Vim2/632-040P2 .06

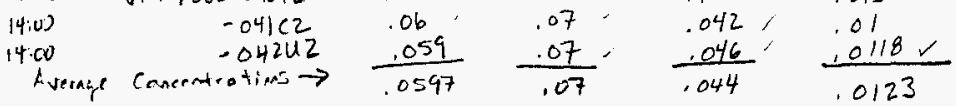

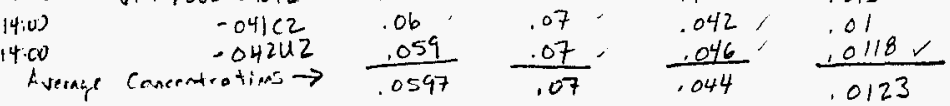

$.112^{2}$

.015

(*) Those concentrations judged to be in error and wat not used in calculations

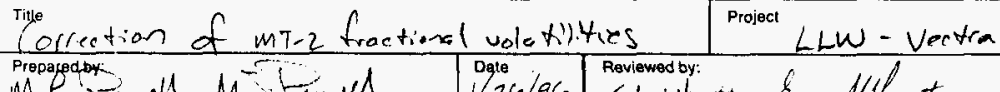

Dale $1 / 26 / 96$ Reviewed by:

E-I7

$2 / 27 / 56$

A. $1007.115(1 / 90)$ 


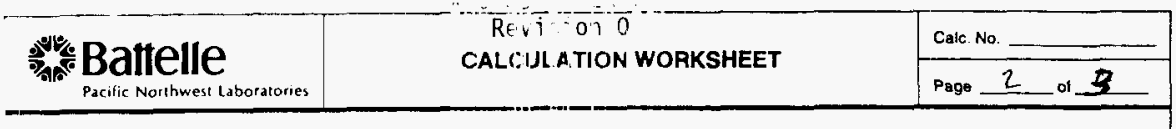

Revised MT -2 volatility rolewiaton for minor components. (cont.)

Sample Calculation:

For the 4/21 $022: 30$ sample,

$$
V_{C 1}=1-\frac{(.05)(.0862)}{(.283)(.0503)}=0.70
$$

Note: - The feed concentrations listed on the preceding page were estimated based on feed makeup records (taken from Appendix 3C of the Vectra report, page 3-76). The given feed conconte-tion data or actually estimated "theoretical glass composition" data, but this dow" adversely attest the woletillty calculation.

- Further, the feed concentrations represent steady-state class component concentrations assuming no volatility losses. The fact that the feed concentrating was changing (for the certain minors) is corrected for by using sib as the

The above volatility calculation is made for the remaining dato on the preceding page to give (see attached sprodshect):

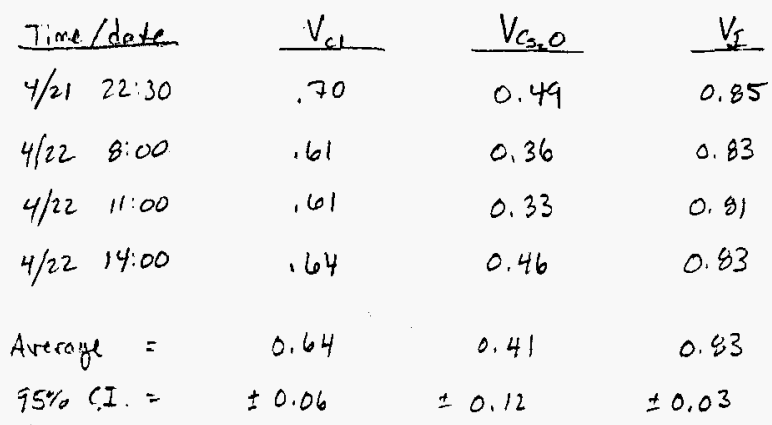

DST DUPABLE COPY

95\% confiders interval is estimated based on the deviation of the 4 calculated values from their mean according to the equation

$$
95 \% \text { CI } \approx \frac{\Sigma_{x} \cdot(\text { student's } t)}{\sqrt{N}}=\frac{\left(S_{x}\right)(3.182)}{\sqrt{4}}
$$

where $S_{x}=$ Estimated population standard deviation

\begin{tabular}{l|l|l} 
Tie & Project \\
Carrion of $m-2$ fractional volatilitios & Vectia
\end{tabular}

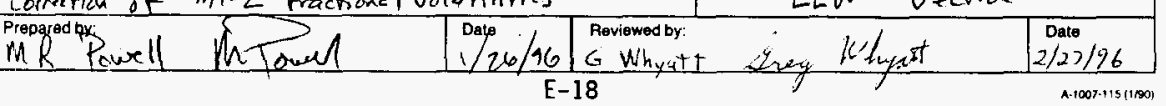


Vectra MT-2

Special Estimate for $\mathrm{I}, \mathrm{Cl}, \mathrm{Cs}$

Based on SrO Tie Calculation 3/14/96 4:36 PM

\begin{tabular}{|c|c|c|c|c|c|c|c|}
\hline Correction & of Fractiona & Volatility & Estimates fo & or Vectra $\mathbf{M}$ & & & \\
\hline Mass Bala & nce Test. (I & R Powell & $1 / 26 / 96)$ & & & & \\
\hline & & & & & & & \\
\hline & & & & & & & \\
\hline & Feed Com & ositions & (from feed & makeup da & ta, p. 3-76) & & \\
\hline & $\mathrm{I}$ & & & & & & \\
\hline Feed & Sro & $\mathrm{Cl}$ & $\mathrm{Cs} 2 \mathrm{O}$ & 1 & & & \\
\hline Feed & & & & & & & \\
\hline Conc $=$ & 0.099 & 0.3252 & 0.1346 & 0.1213 & & & \\
\hline & Glass Com & ositions & & & & & \\
\hline Sample \# & SrO & $\mathrm{Cl}$ & Cs2O & I & $\mathrm{V} \mathrm{cl}$ & Ves2o & $\overline{V I}$ \\
\hline 1 & 0.0503 & 0.05 & 0.035 & 0.0093 & 0.697388 & 0.488212 & 0.8491 \\
\hline 2 & 0.055 & 0.07 & 0.048 & 0.0118 & 0.612546 & 0.358098 & 0.824897 \\
\hline 3 & 0.055 & 0.07 & 0.05 & 0.0131 & 0.612546 & 0.331352 & 0.805606 \\
\hline 4 & 0.0597 & 0.07 & 0.044 & 0.0123 & 0.643049 & 0.457914 & 0.831847 \\
\hline & & & & & & & \\
\hline & & & & Average $=$ & 0.641382 & 0.408894 & 0.827862 \\
\hline & & & & $95 \% \mathrm{Cl}=$ & 0.063656 & $0 . \overline{120774}$ & 0.028624 \\
\hline
\end{tabular}


WHC-SD-WM-ER-517

Revision 0

This page. intentionally left blank. 
WHC-SD-WM-ER-517

Revision 0

APPENDIX $\mathbf{F}$

REVIEW OF DURATEK TEST REPORT

F-1 
WHC-SD-WM-ER-517

Revision 0

This page intentionally left blank. 
To: $\quad$ Greg Whyatt

Subject: Review of Duratek Test Data

From: Eve Tracey

I examined the Phase I Hanford LLW Melter Tests final report by GTS Duratek along with the provided analytical data.

\section{Results of Volatility Calculations}

Total percent losses were calculated using the difference between the mass of oxides in the feed and the mass of oxides in the glass and by using the total mass of oxides trapped in the offgas equipment. First, assuming $3838 \mathrm{~kg}$ oxides are in the feed and $3688 \mathrm{~kg}$ oxides are in the glass, gives an overall loss of $3.9 \pm 1.6 \%$. (The error was calculated using the USGS data.) Using the offgas data, $5.2 \mathrm{~kg}$ oxides were accumulated in the offgas which was divided by the oxides in the feed, giving an overall loss of $0.14 \pm \%$. (The error was not calculated for the offgas data.) Because both the initial volume of feed and the final glass mass were approximations and were used to calculate the total losses, any error associated with those numbers needs to be considered. Once this is done, the two total losses may be in agreement.

Table 1 lists the component masses for the feed (using recipe, USGS, and Corning data), the masses of the components in the glass, the scrubber, the baghouse, and the filtercake. Note that the recipe and USGS data are close while the Corning data is substantially different. Therefore, in most cases the USGS data was used for comparison except for Mg. In this case the Corning data should be used because no $\mathrm{Mg}$ was found in the feed by USGS, but it was found in the glass.

The fractional volatilities of the glass components were estimated four ways: comparison of the oxides in the feed and the glass, comparison of the oxides in the feed and the glass assuming $\mathrm{SiO}_{2}$ is not volatile, total mass of oxides found in the offgas equipment, and using the emissions data directly from the melter. Because there are three different feed values, Table $2 \mathrm{a}$ uses the recipe feed values, Table $2 b$ uses the USGS feed data, and Table $2 c$ uses the Corning feed data. The emissions data was pulled directly from the report and is shown on all three tables even though the values do not change. Table 2a utilizes the recipe feed data to calculate the fractional volatilities. With or without assuming the $\mathrm{SiO} 2$ tie, negative volatilities were calculated for $\mathrm{Al} 2 \mathrm{O} 3, \mathrm{~B} 2 \mathrm{O} 3, \mathrm{P} 2 \mathrm{O} 5$ and $\mathrm{SO} 3$. The sulfur value in glass was much higher than the recipe value. This may be due to error in the analytical results or due to the separation of sulfate salts in the vitrification process. With or without assuming no SiO2 volatility, the losses calculated using the glass data are higher than the losses using the offgas data.

Table $2 \mathrm{~b}$ utilizes the USGS feed data to calculate the fractional volatilities. Negative volatilities were calculated for NiO. Assuming no silica volatility, negative volatilities were calculated for $\mathrm{B2O} 3$, $\mathrm{Fe} 2 \mathrm{O} 3, \mathrm{~K} 2 \mathrm{O}, \mathrm{Na} 2 \mathrm{O}$, and $\mathrm{NiO}$. The glass value of NiO is three times higher than the USGS and Corning analytical data. Again, the losses using the glass data are higher than the losses using the offgas data. 
Table $2 \mathrm{c}$ utilized the Corning feed data to calculate the fractional volatilities. The Corning data appeared to be inaccurate for several of the components. This created negative volatilities for many of the components and larger errors. This data should not be used except to estimate the MgO loss.

For all three tables, in general, the emissions calculated using ES and VSL data were similar to the percent losses calculated using the offgas masses.

\section{Calculations}

1) Feed: The feed values were calculated using the recipe values, the USGS data, and the Corning data. All three values were normalized to $57.5 \mathrm{wt} \%$ oxides (or $3838 \mathrm{~kg}$ oxides). All three feed data were used to calculated losses. Although the Corning analytical feed data were used to calculate volatilities, in general the Corning data should not used because several values, such as $\mathrm{Al}$, were incorrect. No magnesium was found in the USGS feed analysis but it was found in the glass therefore the Corning magnesium value should be used to report percent losses.

The total mass of oxides $(3838 \mathrm{~kg})$ was calculated using Table $5.3 \mathrm{~b}$. Knowing the approximate slurry volume used $(4500 \mathrm{~L})$, the density, and the wt \% oxides, the total mass of oxides was calculated (see attached calculation sheet). Because the numbers $57.5 \mathrm{wt} \%, 4500 \mathrm{~L}$, and $1.75 \mathrm{~g} / \mathrm{cm}^{3}$ were used to calculate the total oxides, they play an important factor in the mass balance calculations and should be accurate. In Table 5.3b, the feed density (with urea and water) is higher than the feed density of the feed without urea and water. This is not likely since urea and water have lower densities than the feed.

3) Glass: The glass values were calculated by averaging the five Corning samples, normalizing to $100 \%$, and multiplying by $3700 \mathrm{~kg}$. Again, because the value of $3700 \mathrm{~kg}$ is very important to the percent loss calculations, an accurate value should be provided rather than an approximation. Because chromium is a refractory component, the target glass value was used rather than the analytical glass value. The sulfur content of the glass is much higher than expected. Sulfur salts separate from the glass during vitrification, therefore the glass sample analyzed may have had a higher sulfur content than the overall glass.

4) Baghouse: The baghouse values were calculated by assuming that no silica was accumulated in the baghouse, averaging the three used baghouse samples, subtracting the difference between the blank and the used baghouse and multiplying by $13 \mathrm{~kg}$. Because the mass accumulated in the baghouse was not directly reported, the $13 \mathrm{~kg}$ was determined by fitting my calculations to the values for the masses reported in Table 5.8.f The baghouse was precoated with diatomaceous earth.

5) Filter Cake: The filter cake values were calculated from one sample which was normalized to $100 \%$ and multiplied by $1.09 \mathrm{~kg}$. Again, the mass accumulated in the filter cake was not directly reported so the same method as described above was used.

6) Scrubber: The scrubber values were calculated by taking the difference between the first and the last samples and multiplying by the scrubber volume (1000 L). The blowdown volume was not used in the calculation. It was assumed that precipitated materials would be captured on the filter rather than being lost in the blowdown volume. (Sodium hydroxide was used to control the $\mathrm{pH}$ in the scrubber.) The authors of the Duratek report took the difference between the first and the second 
samples, then extrapolated. I did not feel comfortable with this method because many of the elements did not increase steadily throughout the melter run.

7) The fractional volatilities of the glass components were calculated by adding the masses found in the scrubber, baghouse, and filter cake then dividing by the mass in the feed (see "Offgas Masses" in Tables $2 a-c)$. The percent losses determined directly from the offgas emissions were reported directly from table 5.8h (see "Emissions" in tables 2a-c).

8) The fractional volatilities of the glass components were also estimated based on the ratios of the glass and feed oxide concentrations (see "Glass" in Tables $2 a-c$ ).

9) The fractional volatilities of the glass components were also estimated based on the ratios of the glass and feed oxide concentrations and the assumption of zero $\mathrm{SiO} 2$ volatility (see "Glass, $\mathrm{SiO} 2$ tie" in Tables $2 \mathrm{a}-\mathrm{c}$ ).

NOTE: This DSI was revised by G. Whyatt in March 1996 to reflect the use of Corning data rather than Pacific Northwest National Laboratory sample data in calculations. 


\section{Addendum to "Review of Duratek Test Data" by Eve Tracey, 9/25/95 Prepared by Greg Whyatt 3/4/96}

Eve Tracey has left PNNL and is not available to address WHC review comments. A few minor comments were addressed within Eve's writeup using redline text while items requiring more lengthy discussion are included here.

\section{Calculation item \#1)}

To perform the calculations a feed density was needed. A value of $1.75 \mathrm{~g} / \mathrm{cm}^{3}$ for the feed prior to urea addition was selected. This density was provided in Table $5.3 \mathrm{~b}$ of the final report and was used along with other data in that table including an $18 \%$ volume increase for the addition of urea and water, and a total oxide content of $57.5 \mathrm{wt} \%$ prior to water and urea addition to conclude a feed:glass ratio of 1.17 liters $/ \mathrm{kg}$ of glass. The final feed density after water and urea addition was measured by WHC at 1.82 to $1.89 \mathrm{~g} / \mathrm{cm}^{3}$ which is greater than the value measured by Duratek of $1.75 \mathrm{~g} / \mathrm{cm}^{3}$ prior to addition of water and urea. The WHC measured density is believed to be in error based on two observations. First, the actual ratio of feed to glass produced, was reported as 1.22 (pg 5-2 of final report) which is closer to the theoretical 1.17 value than the value predicted by the WHC density of 0.94 liters $/ \mathrm{kg}$. Secondly, the feed density is unlikely to increase with the addition of water and urea due to the lower densities of these materials.

\section{Calculation item \#2)}

The glass analysis values were arbitrarily normalized to a $100 \%$ oxide basis. Duratek opted not to perform this normalization. Before normalization, adjustments were made to account for chrome contamination $(0.1 \mathrm{wt} \%), \mathrm{SO}_{3}$ was added because USGS did not report a result $(0.56 \%)$, and $\mathrm{Cl}$ was added because there was no USGS data $(0.19 \%)$. The average percent of total mass that was accounted for prior to normalization was $95.7 \%$.

\section{Additional Comments Pertinent to chloride Losses}

(comments refer to pages 5-5 and 5-6 of the Duratek final report)

Sulfuric acid scrubbers were used to remove $\mathrm{NH}_{3}$ from the sample gas at the melter exit prior to analysis for NOx in the chemiluminescent analyzer. This gas was drawn through a heated filter, scrubbed in the sulfuric acid solution and then transported to the gas analysis trailer and analyzed. During the melter test is was requested that the filter and scrubber solution be analyzed to estimate the melter chloride emissions. Particulates should be deposited on the filter and $\mathrm{HCl}$ would show up in the scrub solution while any $\mathrm{Cl}_{2}$ would not be detected. Capture of $\mathrm{HCl}$ in a sulfuric acid scrub solution would be expected. In fact, capture of $\mathrm{HCl}$ in a $0.1 \mathrm{~N}$ sulfuric acid scrubbing solution is the basis of 40CFR60 app A, Method 26 "Determination of Hydrogen Chloride from Stationary Sources". The Duratek final report contains fundamental errors with respect to this data.

Duratek Final Report, pg 5-5 par 3 states:

"Lastly, analysis of these solution permits an estimate of particulate fluorides and chlorides to be made. Acidic gases such as $\mathrm{HCl}$ and $H F$ would not have been trapped in this strongly acidic 


\section{WHC-SD-WM-ER-517 \\ Revision 0}

scrubbing solution, however particulates such as $\mathrm{NaCl}$ might have been retained."

Duratek Final Report, Pg 5-6 par 3 states:

"Particulate halide salts were only detected at the melter exhaust location, as would be expected. VSL measurements using electrochemical sensors showed the presence of $\mathrm{HCl}$ in the final emissions but no chloride was detected in the sulfuric acid scrubbing solution. This supported the notion that particulate chlorides, not $\mathrm{HCl}$, were detected in the acid scrubbing solutions."

The errors in this text include:

1)Based on the sampling train, there should be no particulates transported to the scrub solution so analysis of the scrub solution will not provide information on particulates.

2) $\mathrm{HCl}$ should be captured by the sulfuric acid scrub solution.

3) Analysis of the filter was not reported so it is not known if any data exists on particulate chloride emissions from the melter.

4) Data in Table $5.7 \mathrm{~h}$ indicates an air concentration at the melter exit of $83 \mathrm{mg}$ chloride/ $\mathrm{m}^{3}$ based on the chloride collected in the sulfuric acid scrub solution. Therefore, it appears that the lack of detection statement applies to the sulfuric acid scrub solutions from downstream of the offgas scrubber. The lack of detection directly conflicts with the reading on the electrochemical $\mathrm{HCl}$ analyzer and does not support the chloride being detected at the melter exit being particulate.

Chloride losses are reported in the Duratek Final Report based on an electrochemical (pg 5-6) offgas analyzer. It is my opinion that none of the data from this instrument are of any value. Concentrations from this analyzer averaged $55 \mathrm{ppm} \mathrm{HCl}$ (pg 8-40). The concentrations reported passing the scrubber are much higher than would be expected. The reason for the error in the instrument is not clear but may relate to interference, drift or other problems.

Probably of greater value is the analysis of the sulfuric acid scrub solutions from the downstream locations. Both the post demister, and post HEPA measurements resulted in air concentrations $<1 \mathrm{mg} / \mathrm{m}^{3} \mathrm{Cl}$ (pg 5-41). The $55 \mathrm{ppm}$ on the CEM suggests a concentration of about $90 \mathrm{mg} / \mathrm{m}^{3}$ $\mathrm{Cl}$. Thus, it is concluded that the scrub solution result is more likely correct and the CEM is more likely incorrect.

Very little about chloride losses can be concluded based on the offgas scrubber. Analysis results for chloride in the scrubber solution were very erratic and the solids collected were not analyzed for $\mathrm{Cl}$.

The sulfuric acid scrub solution at the melter exhaust indicates about $7 \%$ of the feed chloride exits as $\mathrm{HCl}$. Duratek reports 5\% "particulate" (pg 5-6). The reason these values do not agree is not clear although it is noted that there is significant uncertainty due to the poor characterization of the offgas flowrate at the melter exit. 
The analysis of the glass and feed indicates that about $1 / 2$ of the chloride is lost and this result is believed to be valid. Approximately $7 \%$ of the chloride is lost as $\mathrm{HCl}$ (based on analysis of sulfuric acid scrub solution). It is highly likely that during sampling a significant fraction of the chloride withdrawn from the melter exhaust existed as particulate and was deposited on the filter (based on difference between feed/glass analysis calculated losses and losses as $\mathrm{HCl}$ in the scrub solution).

\section{Sample Identification Aid}

The tables attached provide summary results of the mass balance, tie component and emission measurements. This section is intended to improve traceability of the results to the analytical samples on which results are based.

Table 1.

USGS analytical feed is based on samples D1F3-111U, -112U, and $-114 \mathrm{U}$.

Corning analytical feed is based on samples D1F3-111C, 112C, 114C.

Scrubber results are based on D106-101P and -148P.

Baghouse results are based on D107-101C, $-102 \mathrm{C},-103 \mathrm{C}$.

Filter Cake results are based on sample D1010-101C(d).

Glass results are based on Corning glass samples D1G4-108C5, $-109 \mathrm{C} 6,-110 \mathrm{C} 7,-112 \mathrm{C} 8$, and $-115 \mathrm{C} 9$. The normalized concentration is then applied to a glass quantity of $3700 \mathrm{~kg}$.

Table 2a.

"Glass $\mathrm{SiO} 2$ Tie" is based on corning glass results and "recipe feed" vlues in Table 1.

"Offgas Masses" is based on scrubber, baghouse and filtercake from Table 1 compared to the recipe composition with $3838 \mathrm{~kg}$ of glass.

"Glass" is based on recipe feed in Table 1, and corning glass analysis in Table 1. The total mass of feed is $6675 \mathrm{~kg}$ total ( $3838 \mathrm{~kg}$ oxide at $57.5 \mathrm{wt} \%$ oxide) and glass is $3700 \mathrm{~kg}$. Loss is then calculated for each component as $1-(\mathrm{kg}$ in glass $) /(\mathrm{kg}$ in feed).

"Emissions" are based on aerosol sampling results.

Table $2 b$.

" $\mathrm{SiO}_{2}$ Tie" is based on USGS analytical feed in Table 1 and on Corning glass samples in Table 1. "Offgas Mass" is based on scrubber, baghouse and filter cake samples from Table 1 compared to the USGS feed composition applied to 6675 total mass feed ( $3838 \mathrm{~kg}$ feed oxide).

"Flowsheet" is based on the Corning glass analysis from Table 1 compared to USGS feed samples in Table 1. It is assumed that there are $3700 \mathrm{~kg}$ glass and $6675 \mathrm{~kg}$ total mass feed with \% loss as 1 -(glass/feed).

Table 2e.

" $\mathrm{SiO}_{2}$ Tie" is based on Corning glass samples in Table 1 along with corning feed samples D1F3 $-111 \mathrm{C},-112 \mathrm{C}$ and $-114 \mathrm{C}$.

"Offgas" is based on scrubber, baghouse and filter cake samples from Table 1 compared to corning feed samples D1F3-111C, $-112 \mathrm{C}$ and $-114 \mathrm{C}$.

"Glass" is based on Corning feed samples D1F3-111C, $-112 \mathrm{C}$ and $-114 \mathrm{C}$, and corning glass 


\section{WHC-SD-WM-ER-517}

Revision 0

analysis in Table 1. The total mass of feed is $6675 \mathrm{~kg}$ total $(3838 \mathrm{~kg}$ oxide at $57.5 \mathrm{wt} \%$ oxide) and glass is $3700 \mathrm{~kg}$. Loss is then calculated for each component as $1-(\mathrm{kg}$ in glass $) /(\mathrm{kg}$ in feed $)$.

"Emissions" are based on aerosol sampling results. 
Table 1

\section{EVE TRACEY}

DURATEC

MASS DISTRIBUTION

\begin{tabular}{|c|c|c|c|c|c|c|c|}
\hline & $\begin{array}{c}\text { Recipe } \\
\text { Feed } \\
\text { (KG) } \\
\end{array}$ & $\begin{array}{c}\text { USGS } \\
\text { Analytical } \\
\text { Feed } \\
(\mathrm{KG}) \\
\end{array}$ & $\begin{array}{c}\text { Corning } \\
\text { Analytical } \\
\text { Feed } \\
(\mathrm{KG}) \\
\end{array}$ & $\begin{array}{c}\text { Scrubber } \\
(\mathrm{KG})\end{array}$ & $\begin{array}{c}\text { Baghouse } \\
\text { (KG) }\end{array}$ & $\begin{array}{l}\text { Filtercake } \\
\text { (KG) }\end{array}$ & $\begin{array}{l}\text { Glass } \\
\text { (KG) }\end{array}$ \\
\hline $\mathrm{Al}_{2} \mathrm{O}_{3}$ & 240.74 & 257.00 & 55.24 & 0.01 & 0.07 & 0.01 & 241.04 \\
\hline $\mathrm{B}_{2} \mathrm{O}_{3}$ & 239.19 & 245.40 & 281.83 & 0.60 & 0.11 & 0.01 & 249.62 \\
\hline $\mathrm{BaO}$ & 0.00 & 0.53 & 0.63 & 0.00 & 0.01 & 0.00 & 0.43 \\
\hline $\mathrm{CaO}$ & 304.50 & 305.86 & 143.32 & 0.02 & 0.04 & 0.05 & 272.58 \\
\hline $\mathrm{Cr}_{2} \mathrm{O}_{3}{ }^{2}$ & 1.55 & 2.68 & 2.64 & 0.02 & 0.02 & 0.00 & 1.55 \\
\hline $\mathrm{Cs}_{2} \mathrm{O}$ & 5.80 & 5.23 & 5.52 & 0.14 & 0.16 & 0.00 & 4.25 \\
\hline $\mathrm{Fe}_{2} \mathrm{O}_{3}$ & 291.36 & 243.62 & 278.36 & 0.01 & 0.41 & 0.08 & 242.12 \\
\hline $\mathrm{K}_{2} \mathrm{O}$ & 143.36 & 133.22 & 152.92 & 0.63 & 0.56 & 0.01 & 132.81 \\
\hline $\mathrm{Li}_{2} \mathrm{O}$ & NA & NA & NA & 0.00 & NA & NA & NA \\
\hline $\mathrm{MgO}$ & 0.00 & 0.00 & 12.01 & 0.00 & 0.00 & 0.00 & 11.60 \\
\hline $\mathrm{MnO}_{2}$ & 0.00 & 6.42 & 5.20 & 0.00 & 0.00 & 0.00 & 4.64 \\
\hline $\mathrm{MoO}_{3}$ & 5.80 & 5.40 & 5.36 & 0.03 & 0.01 & 0.00 & 5.02 \\
\hline $\mathrm{Na}_{2} \mathrm{O}$ & 707.14 & 677.72 & 786.51 & NA & 0.79 & 0.10 & 682.60 \\
\hline $\mathrm{NiO}$ & NA & 0.13 & 0.11 & 0.0000 & 0.0015 & 0.0001 & 0.87 \\
\hline $\mathrm{P}_{2} \mathrm{O}_{5}$ & 7.34 & 9.10 & 7.66 & NA & 0.01 & 0.00 & 10.82 \\
\hline $\mathrm{SO}_{3}$ & 8.11 & NA & NA & 0.42 & NA & NA & 21.52 \\
\hline $\mathrm{SiO}_{2}$ & 1642.28 & 1697.86 & 1857.51 & 0.05 & 0.00 & 0.79 & 1590.93 \\
\hline $\mathrm{SrO}$ & 4.25 & 4.41 & 0.13 & 0.00 & 0.00 & 0.00 & 4.25 \\
\hline $\mathrm{TiO}_{2}$ & 38.64 & 37.81 & 32.29 & 0.00 & 0.00 & 0.00 & 33.09 \\
\hline $\mathrm{ZrO}_{2}$ & 198.23 & 205.46 & 210.57 & 0.00 & 0.00 & 0.00 & 178.03 \\
\hline Carbon & $\mathrm{NA}$ & 187.09 & 178.28 & NA & NA & NA & NA \\
\hline $\mathrm{Cl}$ & 13.52 & 14.62 & 13.08 & 1.42 & NA & $\mathrm{NA}$ & 7.15 \\
\hline $\mathrm{F}$ & 11.21 & 3.75 & 4.80 & NA & 0.12 & 0.00 & 5.10 \\
\hline SUM OF OXIDES & 3838 & 3838 & 3838 & 1.93 & 2.20 & 1.09 & 3688 \\
\hline
\end{tabular}

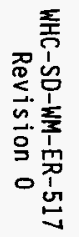




\section{EVE TRACEY}

PERCENT LOSS

\section{DURATEC}

\section{BASED ON Recipe}

\begin{tabular}{|c|c|c|c|c|c|c|c|c|}
\hline & \multirow[b]{2}{*}{$\begin{array}{c}\text { Glass, } \mathrm{SiO}_{2} \text { Tie } \\
(\%)\end{array}$} & \multirow[b]{2}{*}{$\begin{array}{c}95 \% \mathrm{Cl} \\
(+\%) \\
\end{array}$} & \multirow[b]{2}{*}{\begin{tabular}{|c} 
Offgas Masses \\
$(\%)$
\end{tabular}} & \multirow[b]{2}{*}{$\begin{array}{c}95 \% \mathrm{Cl} \\
\text { (not calculated) }\end{array}$} & \multirow[b]{2}{*}{$\begin{array}{c}\text { Glass } \\
(\%)\end{array}$} & \multicolumn{3}{|c|}{ Emissions } \\
\hline & & & & & & $\begin{array}{c}95 \% \mathrm{Cl} \\
(+\%)\end{array}$ & $\begin{array}{c}\text { VSL } \\
\text { (\%Loss) }\end{array}$ & $\begin{array}{c}\text { ES } \\
(\% \text { Loss })\end{array}$ \\
\hline $\mathrm{Al}_{2} \mathrm{O}_{3}$ & -3.4 & 2.1 & 0.0 & & -0.8 & 0.7 & & \\
\hline $\mathrm{B}_{2} \mathrm{O}_{3}$ & -7.7 & 2.8 & 0.3 & & -5.1 & 1.8 & 0.5 & $0.2-0.4$ \\
\hline $\mathrm{BaO}$ & \#DIV/0! & \#DIV/0! & \#DIV/0! & & \#DIV/0! & \#DIV/0! & & \\
\hline $\mathrm{CaO}$ & 7.6 & 2.1 & 0.0 & & 9.9 & 1.1 & & \\
\hline $\mathrm{Cr}_{2} \mathrm{O}_{3}$ & -3.3 & 36.6 & 2.4 & & -0.7 & 35.6 & 2.3 & $1.8-2.1$ \\
\hline $\mathrm{Cs}_{2} \mathrm{O}$ & 24.3 & 1.4 & 5.4 & & 26.1 & 0.0 & 5.5 & $4.9-6.5$ \\
\hline $\mathrm{Fe}_{2} \mathrm{O}_{3}$ & 14.2 & 1.8 & 0.2 & & 16.3 & 0.9 & & \\
\hline $\mathrm{K}_{2} \mathrm{O}$ & 4.4 & 2.0 & 0.9 & & 6.7 & 0.9 & 0.6 & $0-0.2$ \\
\hline $\mathrm{Li}_{2} \mathrm{O}$ & \#VALUE! & \#VALUE! & NA & & \#VALUE! & \#VALUE! & & \\
\hline $\mathrm{MgO}$ & \#DIV/0! & \#DIV/0! & \#DIV/0! & & \#DIV/0! & \#DIV/0! & & \\
\hline $\mathrm{MnO}_{2}$ & \#DIV/0! & \#DIV/0! & \#DIV/0! & & \#DIV/0! & \#DIV/0! & & \\
\hline $\mathrm{MoO}_{3}$ & 10.5 & 1.7 & 0.6 & & 12.7 & 0.0 & 0.8 & $0.4-0.5$ \\
\hline $\mathrm{Na}_{2} \mathrm{O}$ & 0.4 & 1.9 & 0.1 & & 2.8 & 0.4 & 0.3 & $0.1-0.15$ \\
\hline $\mathrm{NiO}$ & \#VALUE! & \#VALUE! & NA & & NA & NA & & \\
\hline $\mathrm{P}_{2} \mathrm{O}_{5}$ & -52.2 & 10.0 & 0.2 & & -48.4 & 9.3 & & \\
\hline $\mathrm{SO}_{3}$ & -173.7 & 19.4 & NA & & -167.0 & 18.2 & & \\
\hline $\mathrm{SiO}_{2}$ & 0.0 & 2.7 & 0.1 & & 2.5 & 1.8 & & \\
\hline $\mathrm{SrO}^{2}$ & -3.3 & 1.9 & 0.0 & & -0.7 & 0.0 & 0.6 & $.08-.1$ \\
\hline $\mathrm{TiO}_{2}$ & 11.6 & 4.1 & 0.0 & & 13.8 & 3.7 & & \\
\hline $\mathrm{ZrO}_{2}$ & 7.3 & 3.0 & 0.0 & & 9.6 & 2.4 & & \\
\hline Carbon & \#VALUE! & \#VALUE! & NA & & NA & NA & & \\
\hline $\mathrm{Cl}$ & 45.4 & 17.7 & 10.6 & & 46.8 & 17.3 & & \\
\hline $\mathbf{F}$ & 53.0 & 12.3 & 1.1 & & 54.2 & 12.0 & & \\
\hline
\end{tabular}


EVE TRACEY

PERCENT LOSS

DURATEC

BASED ON USGS ANALYTICAL RESULTS

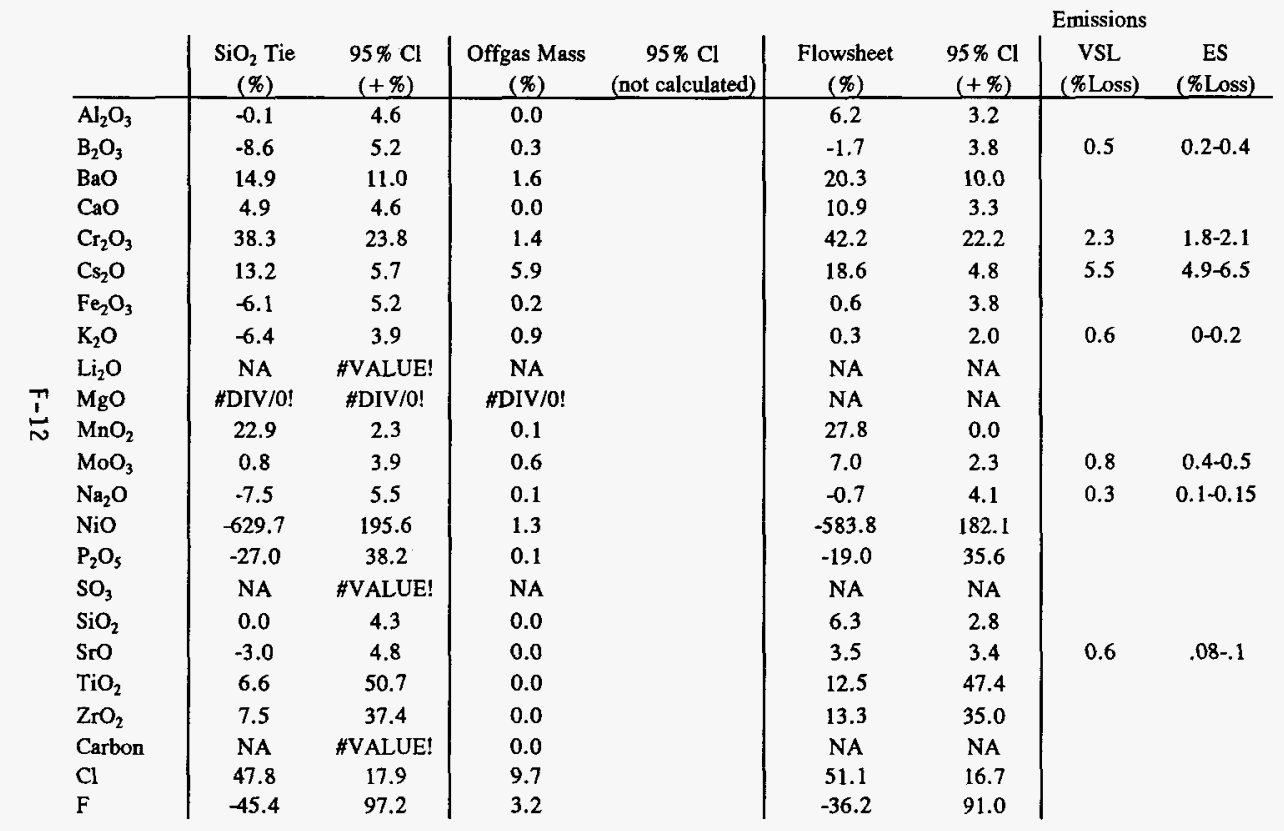


Table 2c

EVE TRACEY

PERCENT LOSS

DURATEC

BASED ON CORNING ANALYTICAL RESULTS

\begin{tabular}{|c|c|c|c|c|c|c|c|c|}
\hline & \multirow[b]{2}{*}{$\begin{array}{c}\text { Glass, } \mathrm{SiO}_{2} \mathrm{Tie} \\
(\%)\end{array}$} & \multirow[b]{2}{*}{$\begin{array}{c}95 \% \mathrm{Cl} \\
(+\%)\end{array}$} & \multirow[b]{2}{*}{$\begin{array}{c}\text { Offgas } \\
(\%)\end{array}$} & \multirow[b]{2}{*}{$\begin{array}{c}95 \% \mathrm{Cl} \\
\text { (not calculated) }\end{array}$} & \multirow[b]{2}{*}{$\begin{array}{c}\text { Glass } \\
(\%)\end{array}$} & \multicolumn{3}{|c|}{ Emissions } \\
\hline & & & & & & $\begin{array}{r}95 \% \mathrm{Cl} \\
(+\%) \\
\end{array}$ & $\begin{array}{c}\text { VSL } \\
\text { (\%Loss) }\end{array}$ & $\begin{array}{c}\text { ES } \\
(\% \text { Loss }) \\
\end{array}$ \\
\hline $\mathrm{Al}_{2} \mathrm{O}_{3}$ & -409.4 & 63.6 & 0.2 & & -336.3 & 16.0 & & \\
\hline $\mathrm{B}_{2} \mathrm{O}_{3}$ & -3.4 & 24.2 & 0.3 & & 11.4 & 17.9 & 0.5 & $0.2-0.4$ \\
\hline $\mathrm{BaO}$ & 21.5 & 38.5 & 1.4 & & 32.8 & 32.0 & & \\
\hline $\mathrm{CaO}$ & -122.1 & 35.4 & 0.1 & & -90.2 & 20.1 & & \\
\hline $\mathrm{Cr}_{2} \mathrm{O}_{3}$ & 31.7 & 28.9 & 1.4 & & 41.5 & 23.8 & 2.3 & $1.8-2.1$ \\
\hline $\mathrm{Cs}_{2} \mathrm{O}$ & 10.1 & 19.4 & 5.6 & & 23.0 & 13.9 & 5.5 & $4.9-6.5$ \\
\hline $\mathrm{Fe}_{2} \mathrm{O}_{3}$ & -1.6 & 21.6 & 0.2 & & 13.0 & 15.3 & & \\
\hline $\mathrm{K}_{2} \mathrm{O}$ & -1.4 & 18.3 & 0.8 & & 13.2 & 11.8 & 0.6 & $0-0.2$ \\
\hline $\mathrm{Li}_{2} \mathrm{O}$ & NA & \#VALUE! & NA & & NA & NA & & \\
\hline $\mathrm{MgO}$ & -12.7 & 94.8 & 0.0 & & 3.4 & 80.3 & & \\
\hline $\mathrm{MnO}_{2}$ & -4.1 & 17.2 & 0.1 & & 10.9 & 10.2 & & \\
\hline $\mathrm{MoO}_{3}$ & -9.4 & 16.9 & 0.6 & & 6.3 & 9.2 & 0.8 & $0.4-0.5$ \\
\hline $\mathrm{Na}_{2} \mathrm{O}$ & -1.3 & 18.9 & 0.1 & & 13.2 & 12.4 & 0.3 & $0.1-0.15$ \\
\hline $\mathrm{NiO}$ & -846.9 & 1049.2 & 1.5 & & -711.0 & 893.4 & & \\
\hline $\mathrm{P}_{2} \mathrm{O}_{5}$ & -65.0 & 75.8 & 0.1 & & -41.3 & 62.7 & & \\
\hline $\mathrm{SO}_{3}$ & NA & \#VALUE! & NA & & NA & $\mathrm{NA}$ & & \\
\hline $\mathrm{SiO}_{2}$ & 0.0 & 16.9 & 0.0 & & 14.4 & 10.2 & & \\
\hline $\mathrm{SrO}$ & -3620.1 & 3232.1 & 0.7 & & -3086.2 & 2742.1 & 0.6 & $.08-.1$ \\
\hline $\mathrm{TiO}_{2}$ & -19.6 & 117.6 & 0.0 & & -2.5 & 99.9 & & \\
\hline $\mathrm{ZrO}_{2}$ & 1.3 & 53.3 & 0.0 & & 15.5 & 44.5 & & \\
\hline Carbon & NA & \#VALUE! & 0.0 & & NA & NA & & \\
\hline $\mathrm{Cl}$ & 36.2 & 22.8 & 10.9 & & 45.3 & 18.4 & & \\
\hline $\mathrm{F}$ & -24.0 & 62.5 & 2.5 & & -6.2 & 52.0 & & \\
\hline
\end{tabular}


WHC-SD-WM-ER-517
Revision 0

Calculations

Feed:

$4500 \mathrm{~L}$ LbW and urea

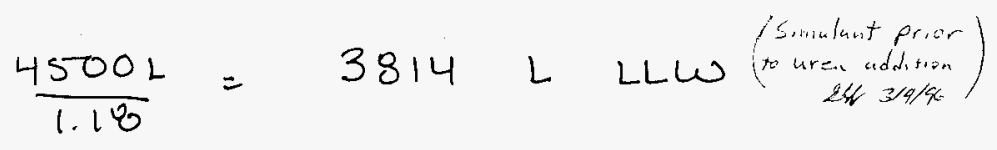

$(3814 \mathrm{~L})\left(1.75 \mathrm{~g} / \mathrm{cm}^{3}\right)=6474.5 \mathrm{~kg}$

$57.5 \omega+\%$ oxides

$(6674.5 \mathrm{~kg})(.575)=3838 \mathrm{~kg}$ oxides

Glass:

$3700 \mathrm{~kg}$ glass

BEST RIRULABLE COPY

Review Frey Why eat $10 / 3 / 45$ 


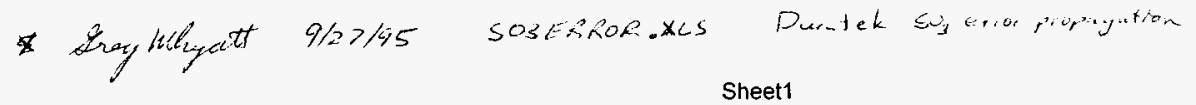

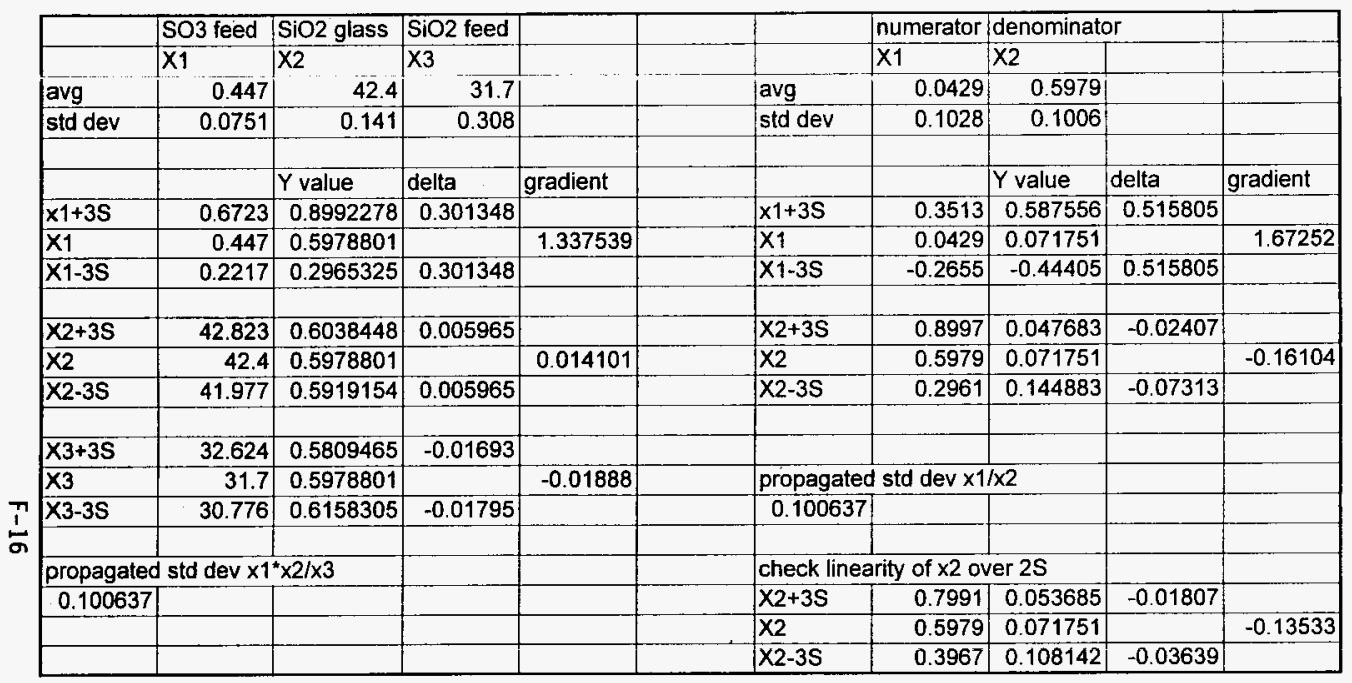

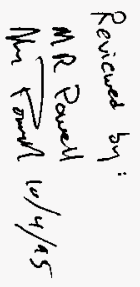


WHC-SD-WM-ER-517

Revision 0

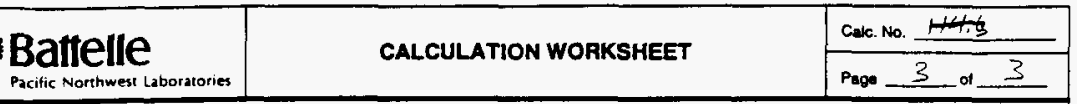

or $\% 655=\frac{.0429 \pm .1029}{.5979 \pm .1006}$

See attached spreadsheet. One to nonlinearity, only linearize over a 20 range.

$$
\begin{aligned}
s+1 \operatorname{ter}=\theta_{1}^{2} \sigma_{1}^{2}+\theta_{2}^{2} \sigma_{2}^{2} & =\left[(1.6725)^{2}(.1028)^{2}+(-.1353)^{2}(.1006)^{2}\right]^{1 / 2} \\
& =\left(2.750 \times 10^{-2}+1.853 \times 10^{-4}\right)^{1 / 2}=.173 .
\end{aligned}
$$

$\therefore$ Std der on calculated result is $17.3 \%$

or $7.2 \% \pm 17.3 \%$ indicating significant uncertainty

Compare $\mathrm{SO}_{2}$ emissions to Source

$\mathrm{SO}_{2}$ CEM data from Environmental Services, Table 6.3 HEPA Exit

$$
\text { Run } \text { R }_{1}=243 \mathrm{pm}
$$

Run $2=245$ Am

How at HEPA exit table 6.2 (Environmental Services)

$$
\begin{aligned}
& \text { Run } * 1 \text { 593 dscfm } \\
& \text { Run } 2 \text { 565 dscfm }
\end{aligned}
$$

assume $s+d=60^{\circ} \mathrm{F}$

Target $\mathrm{SO}_{3}$ (from Table / of Duratek review)

$\mathrm{SO}_{2}=8,11 \mathrm{~kg}$ Total g lase $=3838 \mathrm{~kg} \frac{8,11}{3838}=2.113 \times 10^{-3} \frac{\mathrm{kg} 5 \mathrm{O}_{3}}{\mathrm{kgghas}}$

Total glass production $=1800 \mathrm{~kg} / \mathrm{dar}$

Target Source $\left.\left(1800 \frac{\mathrm{kg}}{\mathrm{d}}\right)\left(2.113 \times 10^{-3}\right)\left(\frac{1000 \mathrm{~g}}{\mathrm{~kg}}\right)\left(\frac{\mathrm{mol}}{32.06+3(16)}\right)\left(\frac{\alpha}{24 h}\right) \frac{\mathrm{h}}{60 \mathrm{~min}}\right)=3.30 \times 10^{-2} \mathrm{mad} / \mathrm{min} 5 \mathrm{O}_{3}$

offers flow, $f_{T}^{3}=\left(1 f_{T}^{3}\right)\left(\frac{28.317 l}{f_{T}^{3}}\right)\left(\frac{273.2}{288.8}\right)\left(\frac{\mathrm{mol}}{22.4 \ell}\right)=1.196 \mathrm{gmal}$

$\mathrm{SO}_{2}$ ran ${ }_{1}=\left(593 \frac{\mathrm{dscf}}{\mathrm{min}}\right)\left(\frac{1.176 \mathrm{gmd}}{\mathrm{f}^{3}}\right)\left(\frac{243 \mathrm{su}}{10^{6}}\right)=.1723 \mathrm{~mol} / \mathrm{min}$

$$
\begin{aligned}
\mathrm{SO}_{2} \text { e } \mathrm{run}_{2} \mathrm{H}_{2}=\left(\mathrm{S65} \frac{\mathrm{dscf}}{\min }\right)\left(\frac{1.196 \mathrm{gmd}}{\mathrm{F}^{3}}\right)\left(\frac{245 \mathrm{SO}}{10^{6}}\right) & =.1656 \mathrm{~mol} / \mathrm{min} \\
\text { Avg } & =.169 \mathrm{~mol} / \mathrm{min}
\end{aligned}
$$

$\therefore$ detected $\mathrm{SO}_{2}>$ feed target quantity

BEST MAILABLE COPY

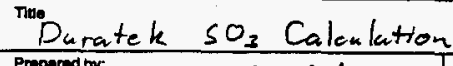

Project Lu Nit

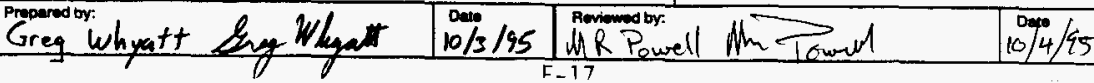




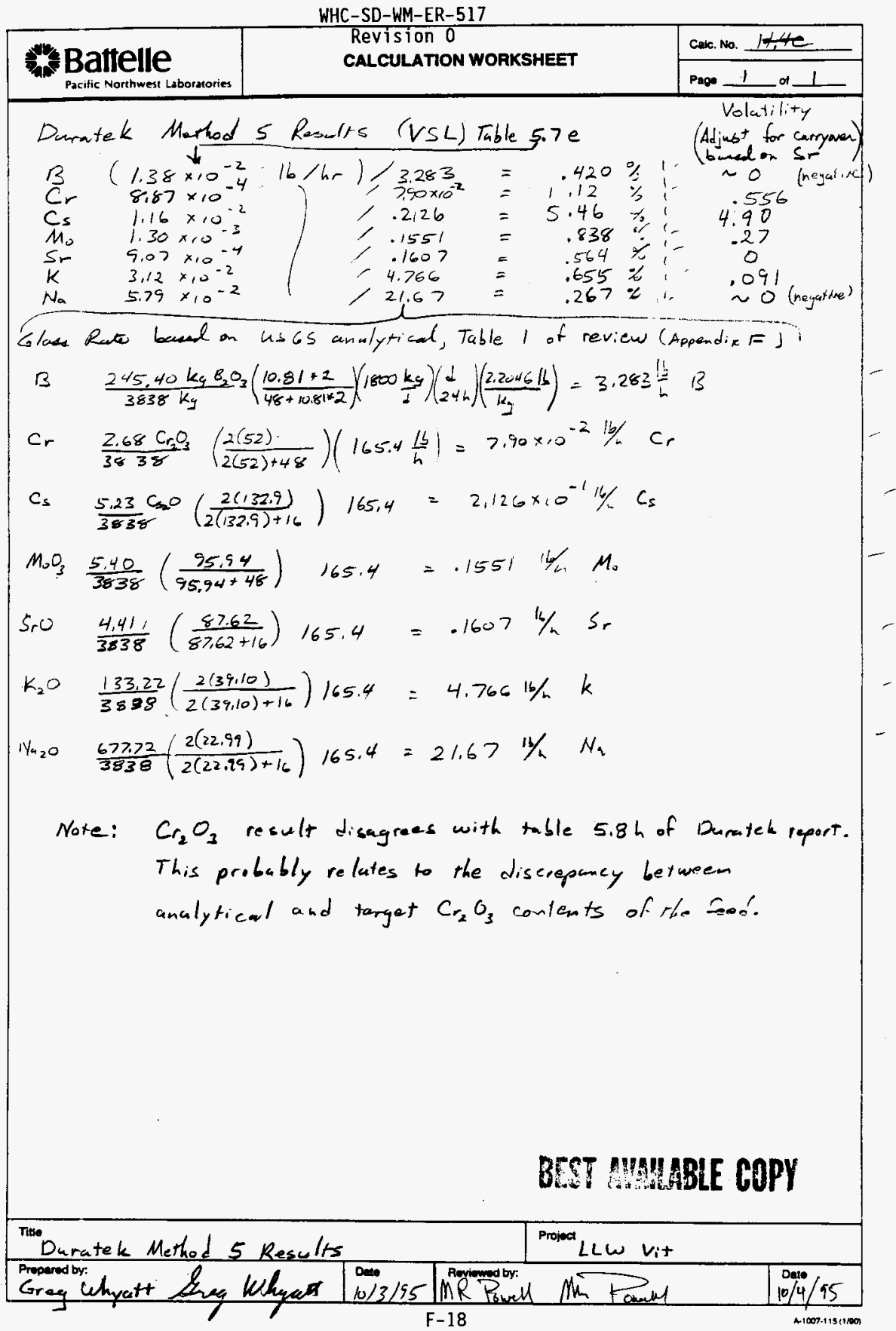


WHC-SD-WM-ER-517

Revision 0

APPENDIX G

REVIEW OF ENVITCO TEST REPORT

G-1 
WHC-SD-WM-ER-517

Revision 0

This page intentionally left blank. 


\section{Envitco, Inc. LLW Melter Demonstration \\ Entrainment and Volatility Estimates (MR Powell 9/1/95) \\ (Modified GA Whyatt 3/21/96)}

The Envitco melter run analytical data were examined and estimates made of the relative volatility and entrainment for each oxide analyte. These analyses and the results are described below. Volatility estimates were made both by comparing the melter feed and glass product compositions and by examining the aerosol collection rates and compositions. Assuming uniform entrainment of feed and zero volatility for a known low volatility component, the fractional volatility of each glass oxide species can be estimated from feed and glass compositions. The entrainment of melter feed was estimated based on the observed average aerosol collection rate on isokinetically-gathered filter samples. Errors were noted in the vendorprovided analysis of the off-gas data and these are described below.

\section{Entrainment}

Feed entrainment between 11:25 and 22:25 on 10/12/94 is estimated to have been on the order of $0.05 \%$ to $0.10 \%$ wt of the feed oxides. During this period of relatively steady operation, the wetted feed pellets were fed to the melter at a rate of approximately $50 \mathrm{lbs} / \mathrm{h}$. Other periods of testing utilized either dried feed pellets or slurry feed, but insufficient off-gas c'iaracterization data are available to evaluate the entrainment of these feed types. The wetted feed pellets were made by adding roughly $7 \%$ wt water to the dried feed pellets. The water was added to suppress dust generation that was noted when the dried feed pellets were fed to the meiter. Entrainment rates for the dried feed pellets and the slurry feed would likely be higher than that observed for the wetted feed pellets.

The $0.05-0.10 \%$ entrainment estimate was developed as follows. Two aerosol samples were collected during the $11-\mathrm{h}$ mass balance period. These samples indicate that the total particulate flow rate was about $0.0345 \mathrm{lbs} / \mathrm{h}$. Over 11 hours, this amounts to about $0.38 \mathrm{lbs}$ of total particulate. During this same period, enough feed was delivered to the melter to produce about $468 \mathrm{lbs}$ of glass assuming no entrainment and zero volatility (p.6-5 Envitco final report). If it is assumed that all of the particulate flow was due to entrainment (i.e., no volatility losses), then the rate of feed entrainment is estimated to be $(0.38 / 468)^{*} 100 \%=0.08 \mathrm{wt} \%$. If there were significant volatility losses of feed oxides the actual entrainment rate would be lower. An entrainment rate of $0.05 \mathrm{wt} \%$ is used for estimating fractional volatilities from aerosol composition data below. Comparing the measured aluminum aerosol flow rate with the aluminum feed rate yields an estimated $0.044 \%$ entrainment rate. These calculations are provided on an attached calculation worksheet.

This low entrainment rate is consistent with the overall mass balance given by Envitco in the final report. A total of $598 \mathrm{lbs}$ of feed was delivered to the melter and a total of $466 \mathrm{lbs}$ of glass was collected during the mass balance run. The feed mass includes $7 \mathrm{wt} \%$ water which was added to reduce dusting. The mass loss on ignition (LOI) for the spray-dried feed was measured 
at $19.1 \mathrm{wt} \%$ on sample E1F3-006U. Envitco chose to average this result with a LOI data point for a feed sample prior to spray drying (E1F2-002U, 24.2\%) to arrive at a LOI for the spray dried feed of $21.7 \mathrm{wt} \%$. Laboratory samples are dried prior to measurement of LOI. However, in the spray dry operation some reaction apparently occurred which destroyed $20-30 \%$ of the nitrate nitrite content of the feed. Although this destruction is much less than hoped for, this destruction is sufficient to make it inappropriate to apply pre-spray dry LOI results to post spray dry material. Thus, a LOI of $19.1 \mathrm{wt} \%$ is believed a superior choice. Adjusting for loss of water and LOI, the expected glass mass is $598(1-0.07)(1-0.191)=450 \mathrm{lbs}$. This is in excellent agreenent with the mass of glass collected $(466 \mathrm{lbs})$. The error could be explained by only a 0.5 inch change in melt level. It is not certain what magnitude of melt level change could be detected.

\section{Volatility}

Fractional volatility estimates were generated for each of the glass oxide analytes. To make these calculations, it is necessary to select a component which is assumed to have zero volatility. Experience has shown that $\mathrm{SiO}_{2}, \mathrm{SrO}, \mathrm{CaO}, \mathrm{Fe}_{2} \mathrm{O}_{3}$ and $\mathrm{Al}_{2} \mathrm{O}_{3}$ are potentially good low volatility tie-components. In this case, $\mathrm{Fe}_{2} \mathrm{O}_{3}$ is not a good tie component because the $\mathrm{Fe}_{2} \mathrm{O}_{3}$ concentration in the glass was systematically increasing during the 11-h mass balance run presumably in response to the change from UN57 batch feed to UN6 batch feed which contained added iron. The $\mathrm{CaO}$ was not selected as a tie component because of the relatively low $\mathrm{CaO}$ concentration in the feed. The results were calculated for the remaining tie components $\mathrm{SiO}_{2}$, $\mathrm{SrO}$ and $\mathrm{Al}_{2} \mathrm{O}_{3}$ and then averaged (see attached spreadsheet). The volatility calculations were made using the average feed and glass composition data after normalization to $100 \%$ on an oxide basis. The variabilities of the feed and glass analyses were used to estimate the $95 \%$ confidence intervals for each oxide concentration. Systematic analytical errors are not included in these uncertainty estimates. The results are given in the attached spreadsheets and summarized in the table below. The NiO concentrations for both glass and feed samples were below the analytical detection limit so no results are presented for $\mathrm{NiO}$.

The tie-component volatilities are within expected uncertainty of zero volatility for most components. The exceptions include $\mathrm{Cr}_{2} \mathrm{O}_{3}, \mathrm{ZrO}_{2}, \mathrm{SO}_{3}$, and $\mathrm{F}$. The $\mathrm{Cr}_{2} \mathrm{O}_{3}$ and $\mathrm{ZrO}_{2}$ sources are attributed to refractory corrosion. Due to analytical difficulties, the target feed concentration (as a ratio to sodium) was used for both chloride and fluoride. The ratio to sodium was considered more accurate than the the overall target because the sodium and $\mathrm{Cl} / \mathrm{F}$ are introduced with the simulant while the feed appeared to be richer in silica than glass target values. The fluoride result indicates a source although no source of fluoride is known in the melter. This result may relate to a deviation from the estimated feed concentration. The final oxide showing a significant loss was $\mathrm{SO}_{3}$ which was based on only 1 glass sample. Two analytical results in glass were available with the higher value being a less than detection limit result. Therefore, only the lower result was utilized. The second glass sample with a less than detection limit glass result would result in an estimate of $>34 \%$ loss of $\mathrm{SO}_{3}$. 
WHC-SD-WM-ER-517

Revision 0

\begin{tabular}{|l|l|}
\hline \multicolumn{2}{|l|}{$\begin{array}{l}\text { Estimate of Waste Oxide Volatilities Based on Tie Component Calculation. } \\
\text { (Average result based on } \mathrm{SiO}, \mathrm{SrO} \text { and } \mathrm{Al} 2 \mathrm{O} \text { tie components.) }\end{array}$} \\
\hline Oxide & Percent Selective Loss (negative indicates gain) \\
\hline $\mathrm{Al}_{2} \mathrm{O}_{3}$ & $-0.5 \pm 7.9$ \\
\hline $\mathrm{B}_{2} \mathrm{O}_{3}$ & $-1.3 \pm 18$ \\
\hline $\mathrm{BaO}$ & $-61 \pm 62$ \\
\hline $\mathrm{CaO}$ & $14 \pm 18$ \\
\hline $\mathrm{Cr}_{2} \mathrm{O}_{3}$ & $-13 \pm 9$ (refractory source) \\
\hline $\mathrm{Cs}_{2} \mathrm{O}$ & $-6.4 \pm 8.8$ \\
\hline $\mathrm{Fe}_{2} \mathrm{O}_{2}$ & $25 \pm 65$ \\
\hline $\mathrm{K}_{2} \mathrm{O}$ & $-1.3 \pm 9.7$ \\
\hline $\mathrm{Li}_{2} \mathrm{O}$ & No Data \\
\hline $\mathrm{MgO}$ & $2.9 \pm 10.7$ \\
\hline $\mathrm{MnO}_{2}$ & $1.3 \pm 69$ \\
\hline $\mathrm{MoO}_{3}$ & $-1.1 \pm 8.9$ \\
\hline $\mathrm{Na}_{2} \mathrm{O}$ & $-0.7 \pm 9.3$ \\
\hline $\mathrm{NiO}$ & All feed and glass results below detection limits \\
\hline $\mathrm{P}_{2} \mathrm{O}_{5}$ & $-10.8 \pm 18.5$ \\
\hline $\mathrm{SO}_{3}$ & $52.7(1$ glass sample, no error estimate possible) \\
\hline $\mathrm{SiO}_{2}$ & $0.8 \pm 7.6$ \\
\hline $\mathrm{SrO}$ & $-0.3 \pm 7.8$ \\
\hline $\mathrm{TiO}_{2}$ & $-6.9 \pm 16$ \\
\hline. $\mathrm{ZrO}_{2}$ & $-441.6 \pm 155$ (refractory source) \\
\hline $\mathrm{Cl}^{-}$ & $0.2 \pm 8.0$ (based on feed target) \\
\hline $\mathrm{F}^{-}$ & $-9.0 \pm 7.3$ (based on feed target) \\
\hline
\end{tabular}




\section{WHC-SD-WM-ER-517 \\ Revision 0}

Based on the tie-component method, it cannot be stated whether any of the analytes other than $\mathrm{SO}_{3}$ exhibited a non-zero volatility during the 11-hour mass balance period. The $\mathrm{SO}_{3}$ result is consistent with a second less than detection limit glass sample, as well as on-line monitors measuring $\mathrm{SO}_{2}$ emissions. $\mathrm{SO}_{2}$ emissions from the melter account for $37 \%$ of the feed sulfur content. In addition to $\mathrm{SO}_{2}$, there may be additional sulfur as filterable aerosols. Therefore, the result from the tie component calculation is believed consistent with the gas monitoring result.

An alternative method for estimating the volatilities of feed oxides is to examine the composition of the collected aerosol samples. Two filter samples were collected during, the 11 hour mass balance period. The samples were weighed by Trigon Engineering Consultants and provided to Quanterra for analysis to provide an estimate of the flow rate of each analyte to the off-gas system. The average of the two reported emission rates for each analyte was taken to be representative of the entire 11-hour run. Based on the average reported loss rate, the total mass loss of each analyte was computed and then compared to the mass of each analyte fed to the melter. The wt\% of each analyte reported by Trigon lost to the off-gas line is shown in the table below. The spreadsheet used to generate these estimates is attached. Also shown in the table is the estimated volatility for each analyte based on an assumed feed entrainment rate of $0.05 \%$ wt. This entrainment rate was estimated from the fractional weight loss of alumina (0.044) and ferric oxide (0.055). Data for silica were not reported and the calcium oxide analytical data are suspect, so neither $\mathrm{SiO}_{2}$, or $\mathrm{CaO}$ can be used to provide entrainment estimates. Sulfur emissions were not quantified as part of these aerosol measurements. 
WHC-SD-WM-ER-517

Revision 0

\begin{tabular}{|c|c|c|}
\hline \multicolumn{2}{|c|}{ Estimate of Waste Oxide Volatilities Based on Aerosol Composition } \\
\hline Oxide & Weight \% of Total Mass Lost & $\begin{array}{c}\text { Estimated volatility (\%) } \\
\text { (assumes 0.05\% wt } \\
\text { Entrainment) }\end{array}$ \\
\hline $\mathrm{Al}_{2} \mathrm{O}_{3}$ & 0.044 & 0 \\
\hline $\mathrm{B}_{2} \mathrm{O}_{3}$ & 0.186 & 0.14 \\
\hline $\mathrm{CaO}$ & $17.2^{*}$ & $17.1^{*}$ \\
\hline $\mathrm{Cr}_{2} \mathrm{O}_{3}$ & 0.022 & 0 \\
\hline $\mathrm{Cs}_{2} \mathrm{O}$ & 0.641 & 0.6 \\
\hline $\mathrm{Fe}_{2} \mathrm{O}_{3}$ & 0.055 & 0 \\
\hline $\mathrm{K}_{2} \mathrm{O}$ & $<0.048$ & 0 \\
\hline $\mathrm{MnO}_{2}$ & 0.179 & 0.13 \\
\hline $\mathrm{MoO}_{3}$ & 0.027 & 0 \\
\hline $\mathrm{Na}_{2} \mathrm{O}$ & 0.016 & 0 \\
\hline $\mathrm{P}_{2} \mathrm{O}_{5}$ & $<0.056$ & 0 \\
\hline $\mathrm{SrO}_{2}$ & 0.146 & 0.10 \\
\hline $\mathrm{Cl}^{-}$ & $1.0-13.4$ & $0-13.4$ \\
\hline $\mathrm{F}^{*}$ & $0.0-0.90$ & 0.85 \\
\hline
\end{tabular}

* This value is probably too high. See discussion in text following table.

Included in the table above are weight loss and volatility estimates for chlorine and fluorine. These values were determined from similar offgas sampling data using Method 26A. The calculations supporting the $\mathrm{F}$ and $\mathrm{Cl}$ values are given on the attached calculation worksheets. A range of values is given for both $\mathrm{F}$ and $\mathrm{Cl}$ because some of the analyses were reported as below detection limit. The \% weight loss range for $\mathrm{F}$ and $\mathrm{Cl}$ was determined by assuming all values below detection limit were zero (establishing the lower limit of the range) or assuming they were equal to the detection limit (establishing, the higher limit of each range).

The volatility estimate for $\mathrm{CaO}$ is given in the table at $17.1 \mathrm{wt} \%$. This is clearly too high based on previous experience. It has been established that $\mathrm{CaO}$ has a very low volatility. The $17.1 \mathrm{wt} \%$ estimate is likely the result of a high blank value for $\mathrm{CaO}$, a failure to correct for the Method 29 blank analysis (see discussion under errors), and the fact that the $\mathrm{CaO}$ concentration 
in the Envitco feed was considerably lower than many of the other vendor tests. Considering the improbability of a $17.1 \% \mathrm{CaO}$ volatility, it is recommended that zero volatility be assumed for calcium oxide in the Envitco melter. It should be noted, however, that a non-zero volatility result could result from selective entrainment of $\mathrm{CaO}$. No mechanisms for selective $\mathrm{CaO}$ entrainment have been postulated. An extensive review of the aerosol sampling analytical work would be required to resolve whether selective entrainment of $\mathrm{CaO}$ is occurring.

\section{Discussion of Errors in Final Envitco Report}

As part of the above entrainment and volatility calculations it was necessary to carefully review the aerosol characterization information provided by Trigon in their report which is included as appendix $\mathrm{G}$ of the Envitco final report. During this review, several calculation errors and inconsistencies were noted. These are documented below. In view of these errors, it is recomrnended that the entire Trigon report be carefully reviewed to ensure there are no additional errors.

On page G-9 a summary table is presented giving the "Multiple Metal Sampling" data obtained from each of the 4 isokinetic aerosol samples. The "Metal Emission Rate" data given for the second filter sample are in error. The total "Particulate Emission Rate" is calculated correctly to be $0.043 \mathrm{lbs} / \mathrm{hr}$, but the emission rates for each of the specific metals aie incorrect. The error, it seems, resulted from the use of the "Run 1 " column of total metal mass data given on page G-28 for the calculation of the respective "lbs/hr" data for both the "Run 1 " and "Run $2^{\text {" }}$ columns in the table on page G-29. Only the "Run 2" column is in error. The data columns for runs 1, 3, and 4 appear to be correct. The corrected data for the "Run 2" column on page G-29 (and the same data on page G-9) are given in the table below. This correction also applies to Table 4.6 on page $4-11$. 
WHC-SD-WM-ER-517

Revision 0

\begin{tabular}{|l|l|}
\hline Metal Species & Run 2 Emission Rate $(\mathrm{lbs} / \mathrm{hr})$ \\
\hline Chromium & $1.77 \mathrm{E}-06$ \\
\hline Calcium & $2.48 \mathrm{E}-03$ \\
\hline Sodium & $1.06 \mathrm{E}-03$ \\
\hline Iron & $2.93 \mathrm{E}-05$ \\
\hline Boron & $2.58 \mathrm{E}-06$ \\
\hline Cesium & $9.55 \mathrm{E}-05$ \\
\hline Aluminum & $2.71 \mathrm{E}-04$ \\
\hline Potassium & $<2.21 \mathrm{E}-04$ \\
\hline Phosphorus & $<2.21 \mathrm{E}-05$ \\
\hline Manganese & $4.28 \mathrm{E}-06$ \\
\hline Molybdenum & $1.16 \mathrm{E}-05$ \\
\hline Strontium & $5.91 \mathrm{E}-05$ \\
\hline
\end{tabular}

These corrected "Run 2 " values were used when generating, the volatility estimates given above. However, it appears there may be an additional error concerning this data. In the tables on page G-28 and G-29 a footnote states "Note: Values are not blank corrected". The data is then summarized on page G-9 and 4-11 and reported without performing a blank correction. The results of one method 29 field blank are included on page G-33. It is not possible to tell without original source information which (the footnote or the data treatment) is in error. Since not performing the blank correction results in conservatively high losses to the offgas, calculations were performed without the blank correction. However, the emission values adjusted for the method 29 blank are shown in the table below for the 4 isokinetic offgas samples. 
Aerosol Mass Flows of Analytes in lb/hr After Correction for Method 29 Blank Analysis ${ }^{(a)}$

\begin{tabular}{|l|l|l|l|l|}
\hline Analyte & Sample \#1 & Sample\#2 & Sample \#3 & Sample \#4 \\
\hline $\mathrm{Al}$ & $1.63 \mathrm{e}-03$ & $6.93 \mathrm{E}-05$ & $4.77 \mathrm{E}-05$ & $1.30 \mathrm{E}-03$ \\
\hline $\mathrm{B}$ & 0 & 0 & 0 & $7.65 \mathrm{E}-04$ \\
\hline $\mathrm{Ca}$ & $3.03 \mathrm{e}-3$ & $5.16 \mathrm{E}-04$ & 0 & $2.44 \mathrm{E}-03$ \\
\hline $\mathrm{Cr}$ & $3.77 \mathrm{e}-5$ & $1.03 \mathrm{E}-06$ & $7.46 \mathrm{E}-07$ & $9.14 \mathrm{E}-06$ \\
\hline $\mathrm{Cs}$ & $9.30 \mathrm{e}-5$ & $9.55 \mathrm{E}-05$ & $5.49 \mathrm{E}-05$ & $3.01 \mathrm{E}-04$ \\
\hline $\mathrm{Fe}$ & $6.59 \mathrm{E}-05$ & $1.36 \mathrm{E}-05$ & $1.36 \mathrm{E}-05$ & $2.92 \mathrm{E}-04$ \\
\hline $\mathrm{K}$ & $<2.22 \mathrm{E}-04$ & $<2.21 \mathrm{E}-04$ & $3.03 \mathrm{E}-04$ & $1.34 \mathrm{E}-03$ \\
\hline $\mathrm{Mn}$ & 0 & $4.03 \mathrm{E}-05$ & 0 & $2.96 \mathrm{E}-06$ \\
\hline $\mathrm{Mo}$ & $9.61 \mathrm{E}-06$ & $1.16 \mathrm{E}-05$ & $2.12 \mathrm{E}-05$ & $6.54 \mathrm{E}-05$ \\
\hline $\mathrm{Na}$ & $1.39 \mathrm{E}-04$ & $4.75 \mathrm{E}-04$ & $6.79 \mathrm{E}-04$ & $5.56 \mathrm{E}-03$ \\
\hline $\mathrm{P}$ & $<2.22 \mathrm{E}-05$ & $<2.21 \mathrm{E}-05$ & $<2.24 \mathrm{E}-05$ & $5.43 \mathrm{E}-05$ \\
\hline $\mathrm{Sr}$ & $3.92 \mathrm{E}-05$ & $5.91 \mathrm{E}-05$ & $1.34 \mathrm{E}-04$ & $4.77 \mathrm{E}-04$ \\
\hline
\end{tabular}

${ }^{(a)}$ Blank analysis results below detection limits are treated as zero results, samples below detection are calculated at the detection level and indicated as < values. Negative results after blank correction are shown as " 0 " values.

The lack of an expected trend in the data was noted in Table G-9. The table provides values in $\mathrm{lb} / \mathrm{hr}$ units for individual analyte metals as well as a total particulate emission rate. The sum of the metal analytes do not compare in a predictable manner with the total particulate emissions. The total metal emissions and particulate emissions are reproduced below. Samples 1 and 2 correspond to the mass balance period. The data indicate that the total metals and the mass of aerosol collected are not well correlated (i.e. high masses of particulate collection do not result in high mass of metals collected). This indicates either analytical problems or possibly that the nature of the aerosol changed significantly between different sample times. Analytical difficulties are considered the more likely cause.

\begin{tabular}{|l|l|l|l|l|}
\hline & Sample \#1 & Sample \#2 & Sample \#3 & Sample \#4 \\
\hline Total Particulate Emission $\mathrm{lb} / \mathrm{hr}$ & 0.026 & 0.043 & 0.038 & 0.114 \\
\hline Total Metal Emission $\mathrm{lb} / \mathrm{hr}$ & 0.00918 & 0.00659 & 0.00508 & 0.0229 \\
\hline \% of mass accounted for by metals & 35 & 15 & 13 & 20 \\
\hline
\end{tabular}


It is recommended that the analyte mass data given on page G-28 be verified against the aerosol sample chemical analyses. These analyses were apparently performed by Quanterra directly for Trigon and are therefore not included in the compilation of analytical data for the LLW melter tests. It would be useful to have the aerosol sample analytical data included in the Trigon report to maintain traceability of the reported aerosol flow rates.

On page 4-7 of the Envitco report there is a discussion of $\mathrm{NO}_{x}$ generation and conversion rates. The sentence ending "gives an offgas rate of $3.4 \mathrm{~g} \mathrm{NO} / \mathrm{min}^{\prime}$ is incorrect. Multiplying a flow rate of $1.56 \mathrm{~m}^{3} / \mathrm{min}$ by a NO concentration of $5.56 \mathrm{~g} / \mathrm{m}^{3}$ gives an offgas rate of $8.7 \mathrm{~g} \mathrm{NO}_{\mathrm{x}} / \mathrm{min}$. This change results in the "1.31 gN/min" value in the next sentence being changed to $3.20 \mathrm{~g} \mathrm{~N} / \mathrm{min}$. The "indicated nitrate reduction factor" of $76 \%$ given in the next paragraph is apparently correct $((13.0-3.2) / 13 * 100 \%=$ $75 \%$ ). The calculation of $5560 \mathrm{mg} / \mathrm{m}^{3} \mathrm{NO}_{\mathrm{x}}$ from $5000 \mathrm{ppm}$ at $130^{\circ} \mathrm{C}$ and 1 atm should also be checked. Assuming ideal gas behavior and a $50 / 50 \mathrm{~mol} \%$ mixture of $\mathrm{NO}$ and $\mathrm{NO}_{2}$ gives $5.745 \mathrm{~g} / \mathrm{m}^{3} \mathrm{NO}_{\mathrm{x}}$.

\section{Page References in Attached Calculations}

The page references for data in the attached calculations refer to the draft Envitco test report. To adjust the references to the final Envitco report change appendix $D$ to appendix $G$ and add 2 to the page number. For example page D22 becomes G24, D23 becomes G25, etc. 


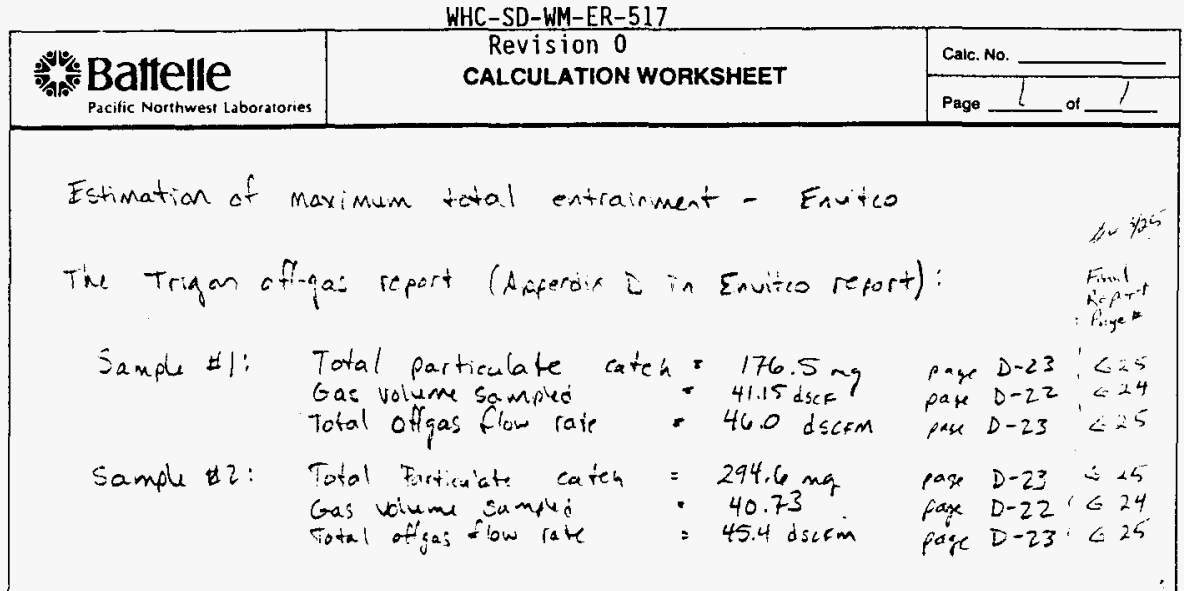

Average particulate flow rate:

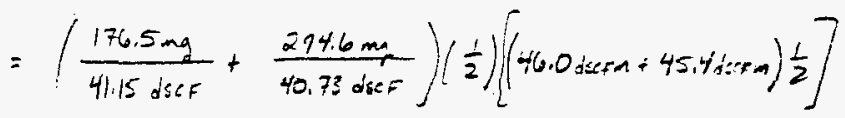

$$
\begin{aligned}
& =263.3 \mathrm{ma} \text { partientet } / \mathrm{min}=15.8 \mathrm{~g} / \mathrm{h} \\
& =0.0348 \mathrm{lb} / \mathrm{h} \text { particalete }
\end{aligned}
$$

over 11 hours, total particulate $=(11)(.0348)=0.383 \mathrm{lbs}$

Total theoretical glass production $\cong 468$ lb: (page $x-8$ Enviteo Report)

Assuming the particulate are in the form of glass oxides, the maximum t entrainment is:

$$
\max E_{n} t=\frac{0.383 l i s}{468 l_{6}} \times 100 \%=0.082 \%<
$$

Because some of the sample mars is likely due to volatilization, the actual entrainment is freckly lower. Assuming Aluminum aerosol flow data on pase $0-27$ an accurate, the entrainmus

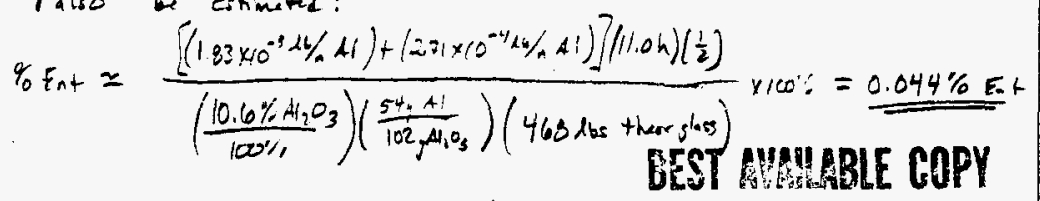

$\therefore$ Actual entrainment B probably about $0.04-0.05 \%$ ut.

File

Project

Prepared by:

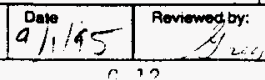

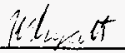

Dato

$\therefore / 4 / 56$ 


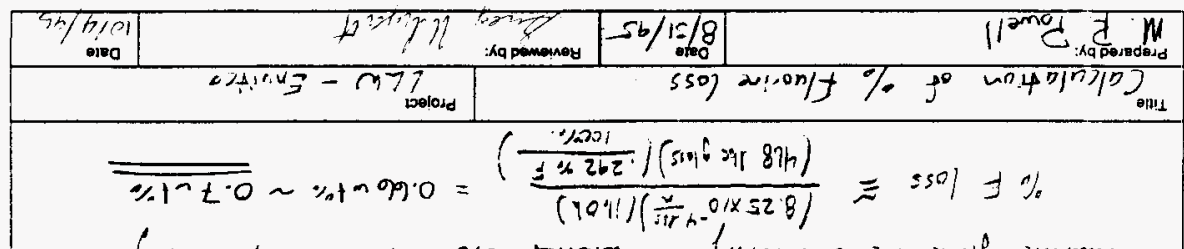

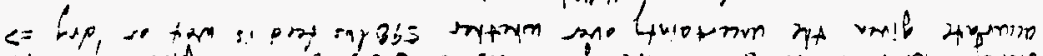

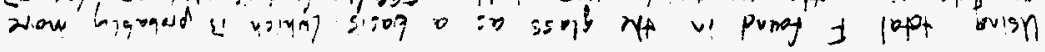

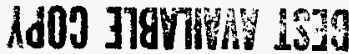

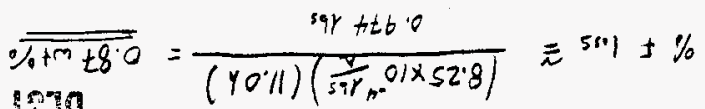

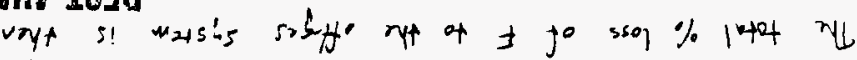

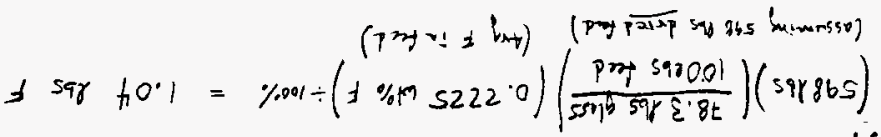

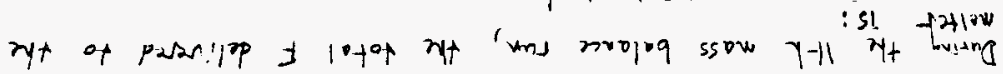

$$
\begin{aligned}
& \sqrt[y]{\operatorname{sir} n^{-01 \times 528}}=14 / 5 s y \quad \Leftarrow \\
& \tau\left(n_{-} 0 \times 99_{b} i+5-01 \times 82 t\right)+
\end{aligned}
$$

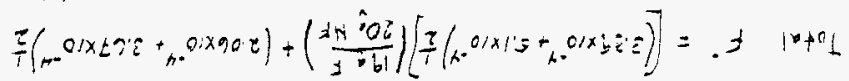

iz of pot mwisz myt s! molf

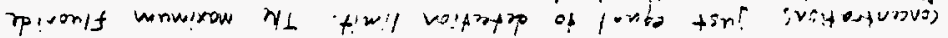

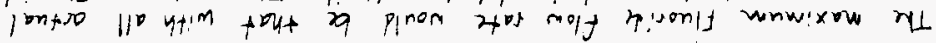

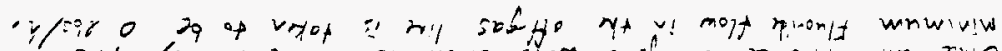

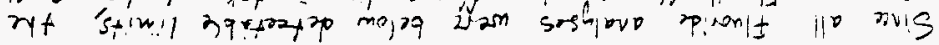

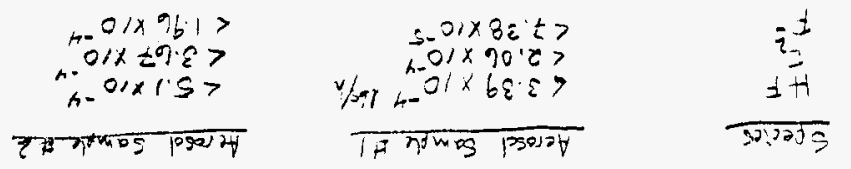

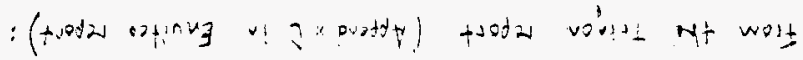

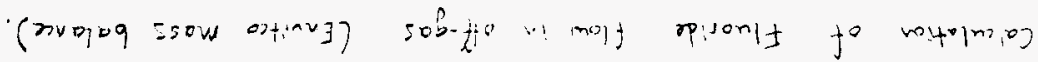




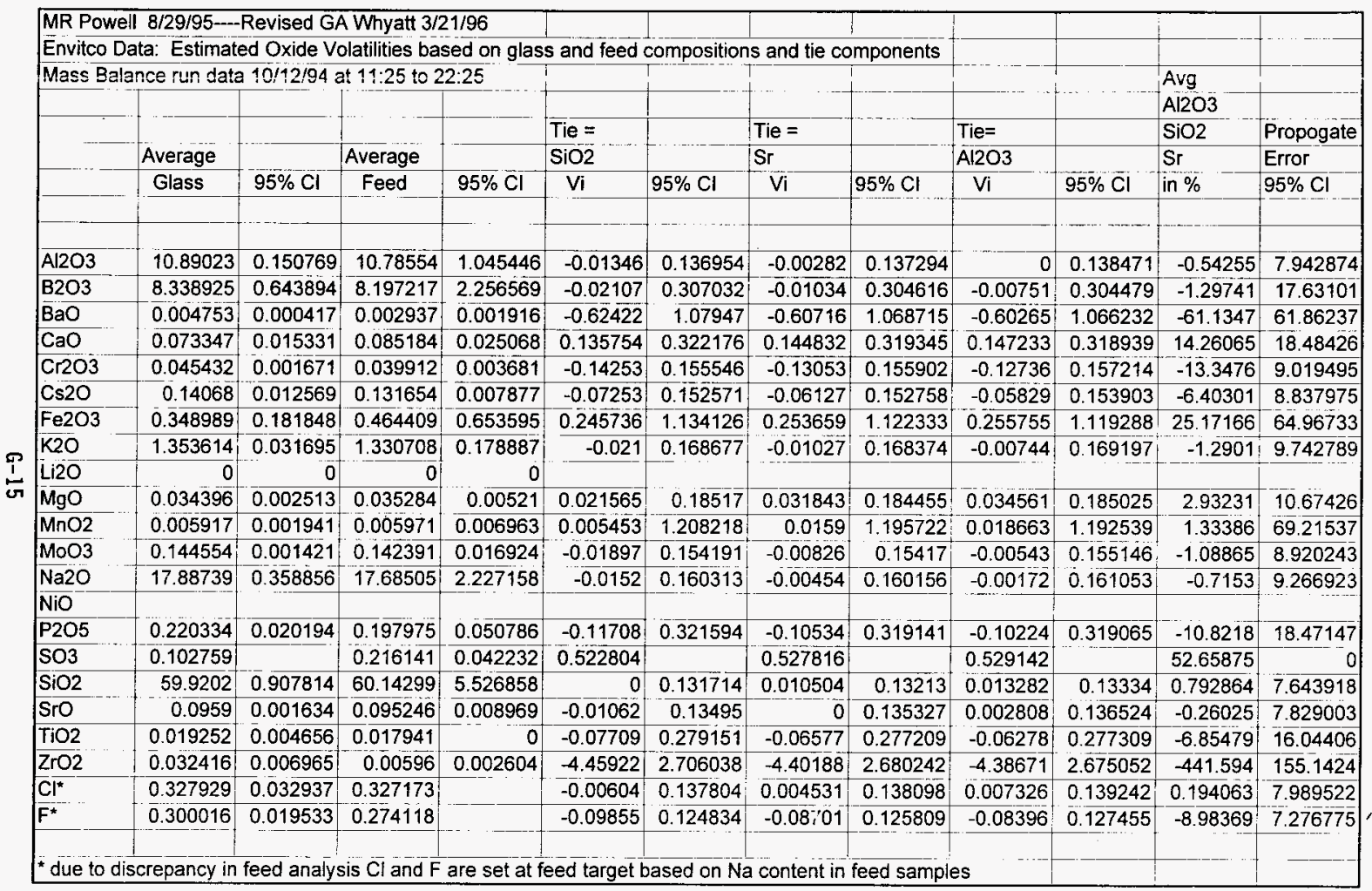

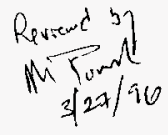


WHC-SD-WM-ER-517

Revision 0

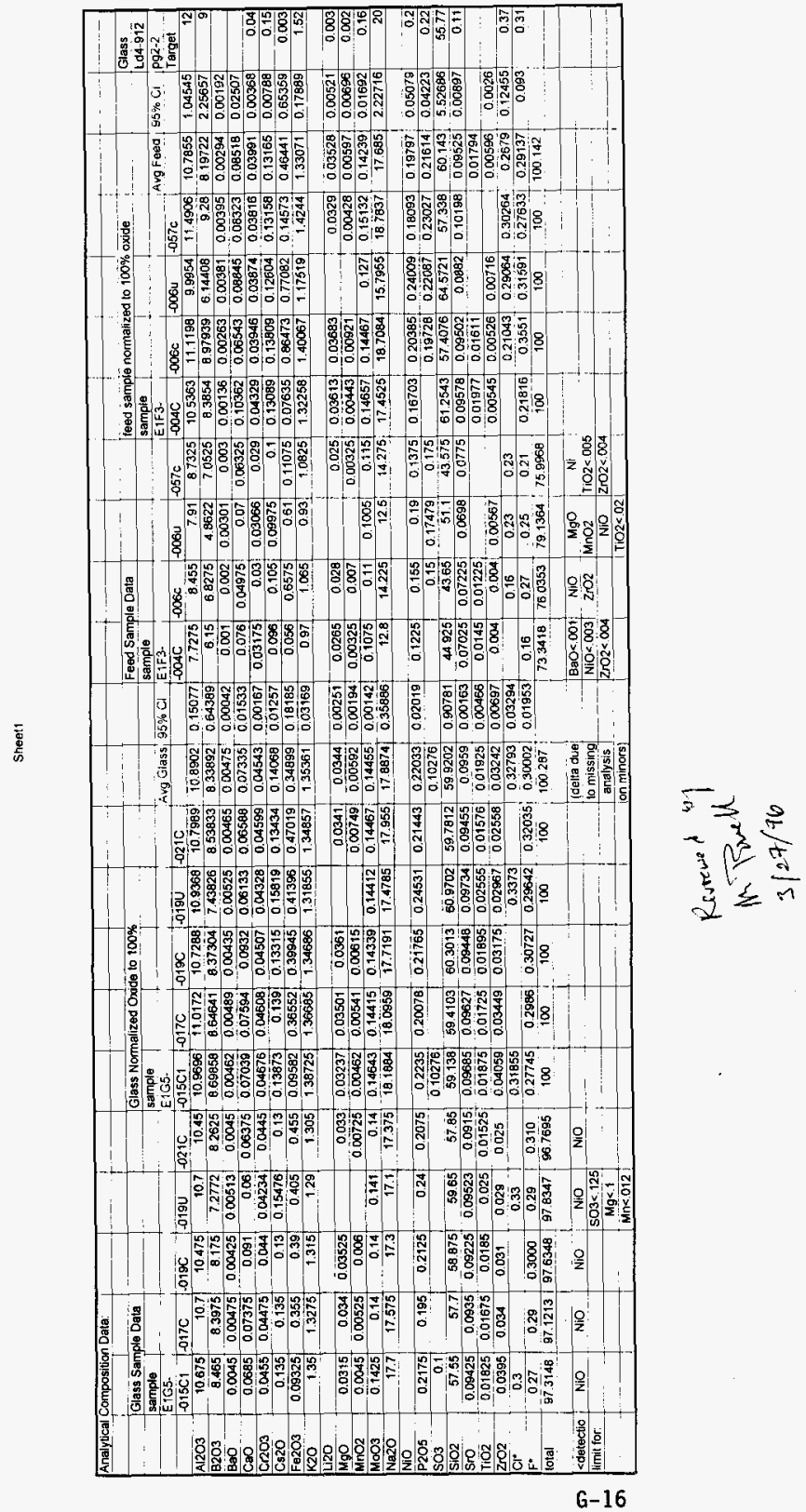




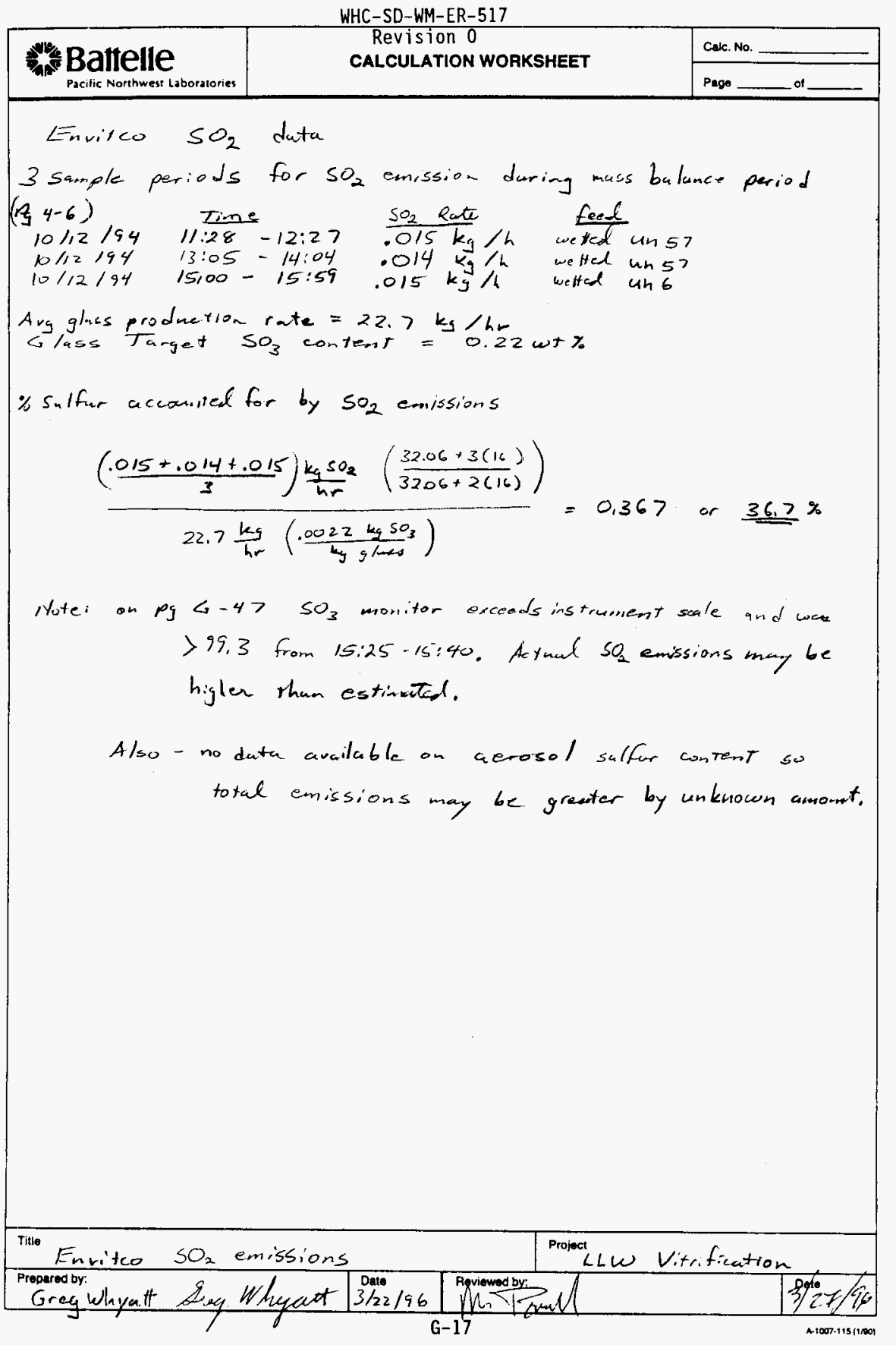


WHC-SD-WM-ER-517

Revision 0

This page intentionally left blank. 
WHC-SD-WM-ER-517

Revision 0

\section{APPENDIX H}

\section{MISCELLANEOUS SUPPORTING CALCULATIONS}

H.l Estimation of $\mathrm{NO}_{x}$ Yields

H.2 Estimation of Iodine Volatility

H.3 Total Loss Calculation 
WHC-SD-WM-ER-517

Revision 0

This page intentionally left blank. 
Revision o

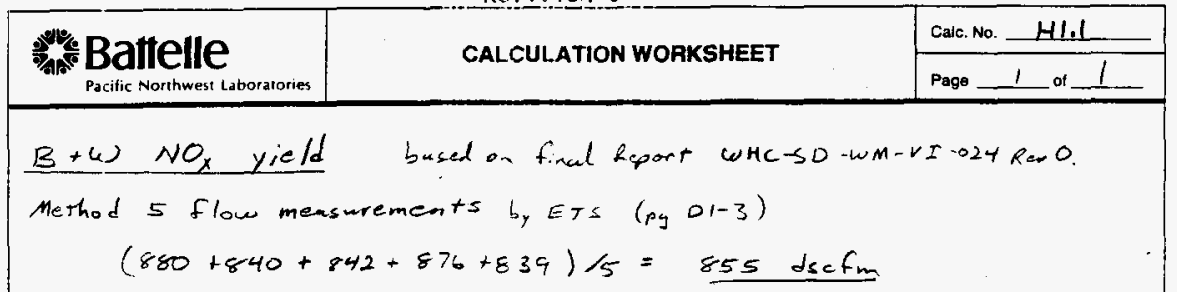

Note: results in Table 5-1 appear inconsistent with flow measwement above results in appendix are as sums correct

Final report appears to be void of any NO x analyzer information. Data from reelimigury report provided the following 5 -minute average data far lox at dry scrubber inlet (See Tables) (dutataken at approx, i hr inter dis)

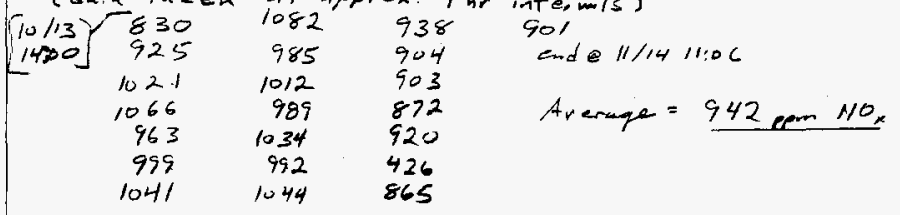

Feed Source

From notes taken during test target feed was $100 \mathrm{lb} / \mathrm{hr}$ mixed feed (Also this rate its consistent with preliminuily data report)

$$
\begin{aligned}
& \mathrm{N}_{3}{ }^{-} \mathrm{NO}_{2}^{-} \mathrm{Content} \text { from Simple analysis } \\
& \text { kIF) }-002 \mathrm{Cl} \text { (s) } 6.82 \text { 2.90 } \\
& \text { BIF7-005C2 (s) 6.56 } 2.79 \\
& B \mid F>-\infty 8<3(5) \quad 7.58 \quad 3.23 \\
& B \mid F=013 C \text { (S) 8.33 } 3.65 \\
& \text { lng } 7.32 \% 3.14 \% \\
& \text { Source }=100 \frac{16}{h}\left(\frac{h}{60 \mathrm{~min}}\right)\left(\frac{453.6 \mathrm{~g}}{16}\right)\left[.0732\left(\frac{\mathrm{mol}}{14+48 \mathrm{gNO}_{3}}\right)+.0314\left(\frac{\mathrm{miN}}{14+32 \mathrm{gNO}_{2}}\right)\right]=1.41 \mathrm{mot} / \mathrm{min}
\end{aligned}
$$

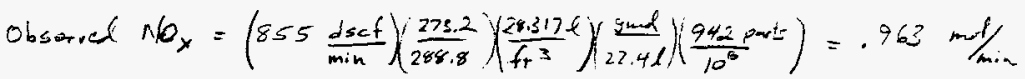

$$
\begin{aligned}
& \text { ie } / d=.963 / 1.41=68 \%
\end{aligned}
$$

Note, Resulsare for no reborn $N_{0}$ INCR

BEST AVaiLABLE COPY

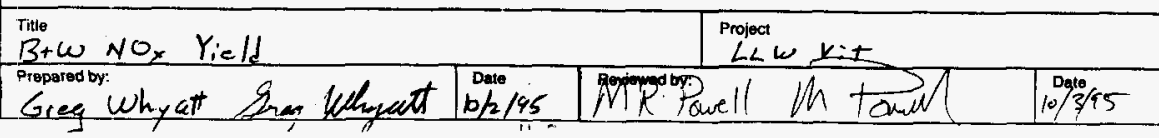


Revisior 0

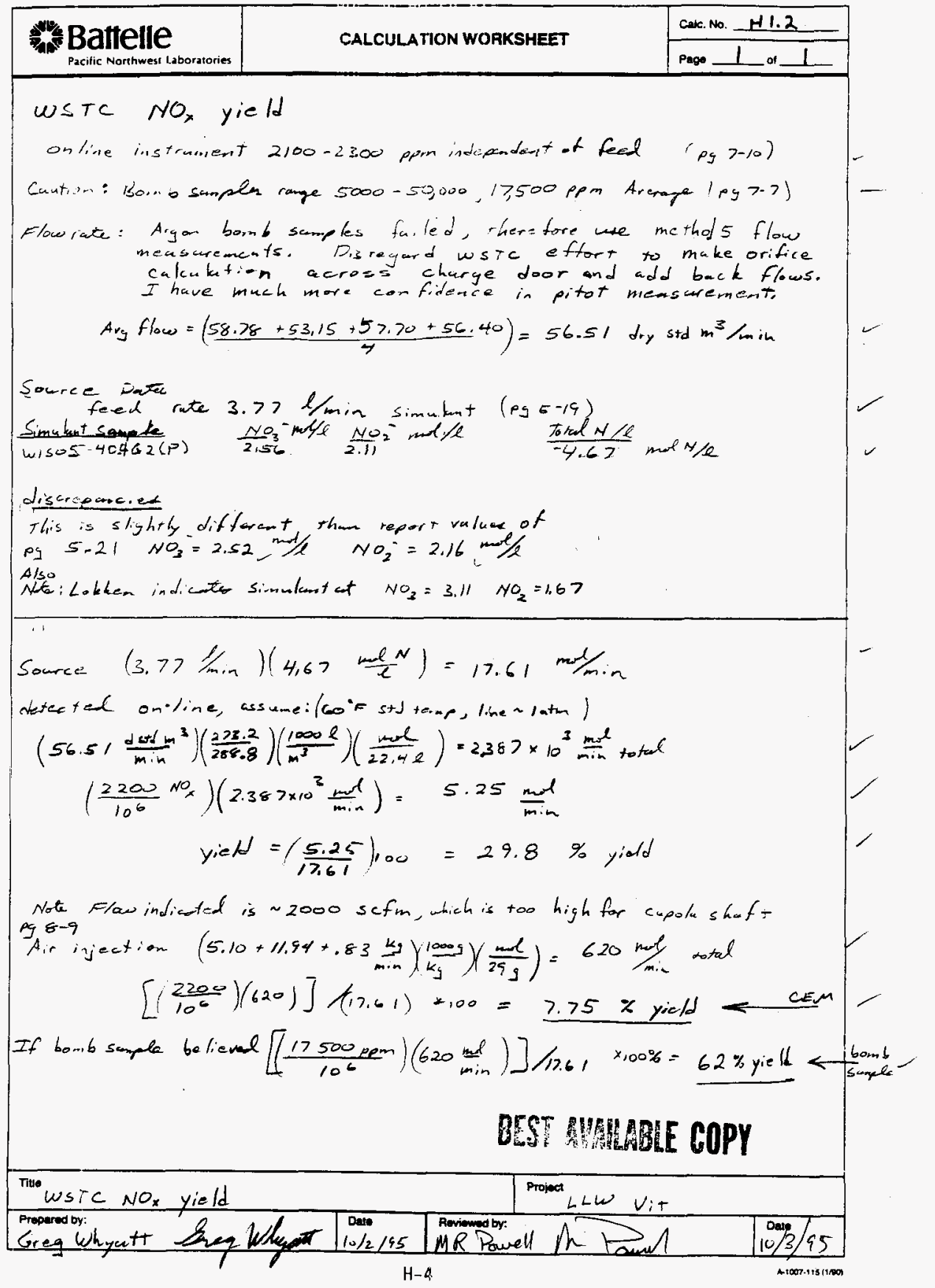


Revisioา 0

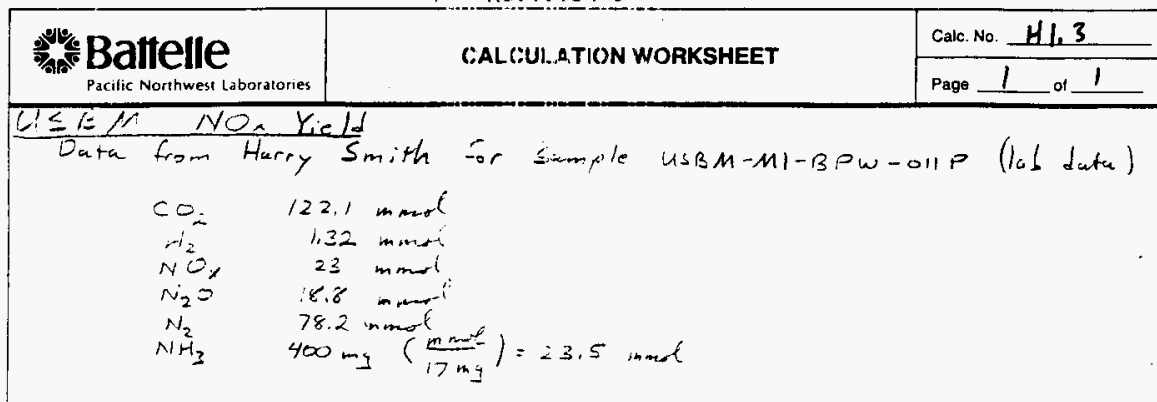

feed prep yicld

$$
\frac{23}{(23+2(18,8)+2(78.2)+23.5}=9.56 \%
$$

Oftyas Data from Malten.

verify that furnuce is 10 t a sugntiand Eactor in AOx yield: pg 24 final report $3 / 9$ e $\sim 22: 25$ feed rate $=750 \mathrm{k} / \mathrm{h}$

Type A feos.', Na candpsis res.lis $=(15.3+15.5+17.6) / 3=16.1$ wr $\% \mathrm{Ala}$

Feed siniaturt target values $\mathrm{Na}_{4}=10 \mathrm{M} \quad \mathrm{NO}_{3}^{-}=3.11 \mathrm{M} \quad \mathrm{NO}_{2}^{-}=1.67 \mathrm{M}$

Entropy leport oy $<48$ approx 2.0 ppren $N O_{x}$ for 6:41-7.41 on $3 / 9$

Entropy Repor pg A 14 cyclone inlet flow (unclene it $\mathrm{Jilwi=mi}$ ) $223 \mathrm{dsct} / \mathrm{m}$ duta taken 3/923:50 only mensurement provided, $5+d=68^{\circ} \mathrm{F}$

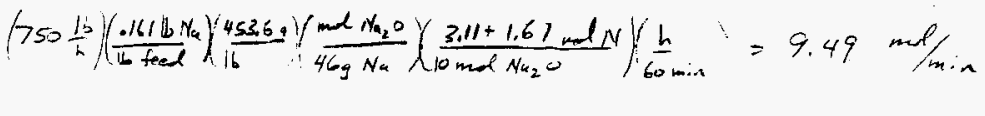

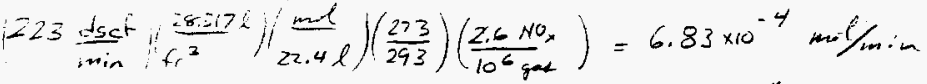

$$
\begin{aligned}
& \%=1 \text { ive ....... } \frac{3.2 .2^{-4}}{9.74} \times 100 \%=.007 \% \\
& \therefore \text { negligible }
\end{aligned}
$$

$$
\text { Nox yiel }=9.6 \%
$$

Title

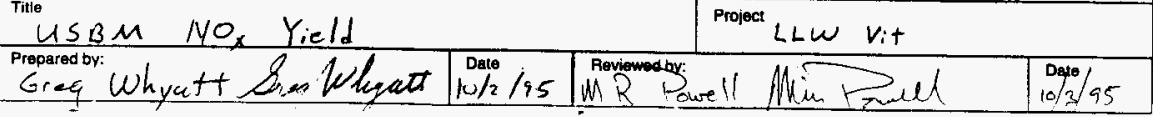


$h^{\prime \prime}-r_{-}-52-h^{2}-5=7-5 \ldots i$

Revision 0

Battelle

\begin{tabular}{|l|l|}
\multirow{2}{*}{ CAL.CUI_ATION WORKSHEET } & CaKc. No. $\frac{H 1.4}{1}$ \\
\cline { 2 - 2 } & Page 1 of 2 \\
\hline
\end{tabular}

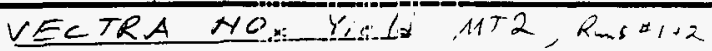

Om+ii report:

$$
\begin{aligned}
& \text { Rum } * 1360 \text { pem 110, !349 pom NO) dr busis } \\
& \text { Elow } 2,35 \text { dscm/m ste } 211 \mathrm{c} \text { latm } \\
& 23: 05 \text { to } 4 / 22 \text { ouros } \\
& \text { Filten }(.700655660)=34.66 \mathrm{my} / \mathrm{dscm} \mathrm{N/O}
\end{aligned}
$$

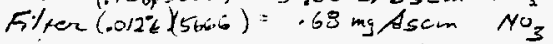

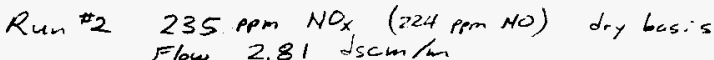

$$
\begin{aligned}
& 4 / 2=11: 56 \quad 4 / 2212: 56 \\
& \text { fitien } .718 \text { \& }(4236)=30.4) \mathrm{mg} / \mathrm{dsam} \mathrm{NO} \\
& .027 \div(4236)=1.14 \mathrm{my} / \mathrm{cscm} \mathrm{Hos}_{3}
\end{aligned}
$$

9323 report $865 \mathrm{~kg}$ slurry fead addel oren 14 hrs consisting of :

$$
141 \mathrm{~kg} \text { butch } \% 1 \text { e } 49.72 \text { woth simulart }
$$

$724 \mathrm{~kg}$ butch 20,39 \& 46.33 utt simulart

Note $57 \%$ by waigh is glues

Appendix 8.3

Mass butmee sodium conclude $10 \mathrm{M}$ simulaht

$$
\text { density } 210 \mathrm{M} \mathrm{Nu}=1.42 \mathrm{~kg} / \mathrm{e} \quad \begin{array}{r}
\mathrm{NO}^{-} \\
\mathrm{NO}_{2}^{-}=3.11 \mathrm{M}
\end{array}
$$

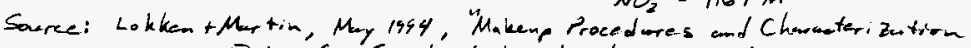
Data for Simulited Lav-level Wastes"

Potential Source of $N$

$$
\begin{gathered}
{\left[141 \mathrm{~kg}(.4972)\left(\frac{l}{1.42 \mathrm{~kg} \operatorname{sim}}\right)\left(3.11+1.67 \frac{\mathrm{ml} N}{l}\right)+724(.4638)\left(\frac{l}{1.42 \mathrm{~kg}_{\mathrm{g}}}\left(3.11+1.67 \frac{\mathrm{kg}}{\mathrm{l}} \mathrm{N}\right)\right]\right.} \\
-/\left(14 \mathrm{hr} \cdot \frac{60 \mathrm{~min}}{\mathrm{~h}}\right)=1.63 \mathrm{~mol} / \mathrm{min} N \text { source }
\end{gathered}
$$

Run 1 Rate

$\left(2.95 \frac{\mathrm{m}^{3}}{\mathrm{~min}} \sqrt{\frac{1000 \mathrm{l}}{\mathrm{m}^{3}}}\left(\frac{\mathrm{gmdl}}{24.1 \mathrm{l}}\right)\left(\frac{360 \mathrm{~N}}{10^{6} \mathrm{tor}}\right)=4.406 \times 10^{-2} \mathrm{mal} \mathrm{N} / \mathrm{min}\right.$ gard

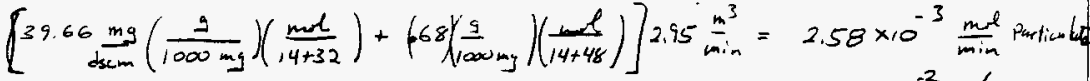

$$
\begin{aligned}
& \text { Total }=4.66 \times 10^{-2} \mathrm{~m} / \mathrm{min} \\
& \text { yie/d }=4.46 \times 10^{-2} / 1.63=2.74 \%
\end{aligned}
$$

MET RPREARE COPY

Theoctra NOX Yield

Proper LL Vit.

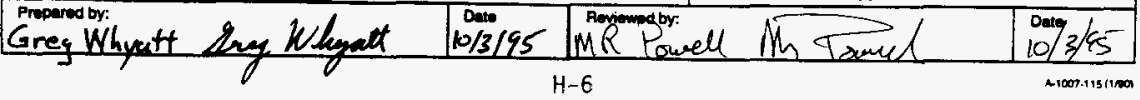


WHC-SD-W/M-ER-517

Revision 0

Battelle

CALCULAITION WORKSHEET

Pacific Northwest Laboratories

Caic. No. $\mathrm{Hl} .4$

$R_{\text {unn }} * 2$ Rate

$$
2.81 \mathrm{~m}^{3} \frac{1000 \mathrm{lgmd} / 235 \mathrm{~N}}{\mathrm{~m}^{2} 24,1 \mathrm{l} 10^{6}}=2.7 .1 \times 10^{-2}
$$

on Method 5

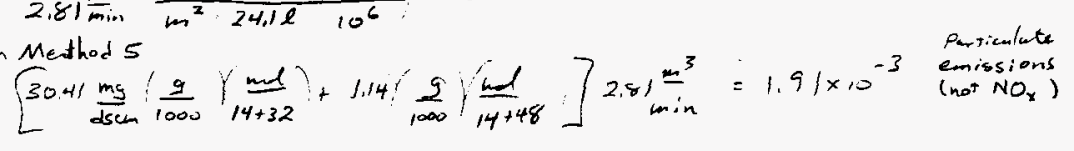

Partimbats yiell

$$
\begin{gathered}
\text { NOx yield }=\frac{2.74 \times 10^{-2}}{1.63}=1.68 \% \text { yield } \\
\frac{1.9 \times 10^{-3}+2.58 \times 10^{-3}}{1.63}=0.28 \% \text { Farticubute } \\
N O_{x} \text { yield Arg }=\frac{2.74+1.68}{2}=2.21 \%
\end{gathered}
$$

Gencref cheak on assumptions bued on Na

$$
\begin{aligned}
& 865 \mathrm{~kg} \text { fad }\left(.57 \frac{\mathrm{kg} / \mathrm{kg}}{\mathrm{kg} \text { feed }}\right)=493 \mathrm{~kg} \text { glase }
\end{aligned}
$$

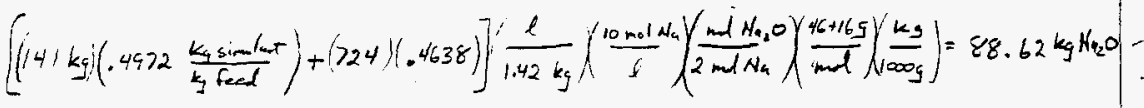

$$
\begin{aligned}
& \text { wt \% } N_{n_{2}} O \text { with no } 100 \%=\frac{\$ 8.62}{493}{ }^{1000}=18.0 \% \\
& \text { Actrad gluss composition } 15.54 \%
\end{aligned}
$$


WHC-SD-AM-EF-5:.7

Revision 0

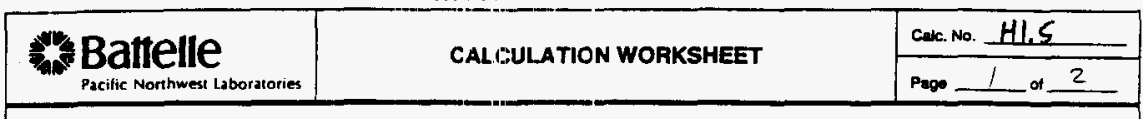

Duratek NOx yield

Claim in 6-6 <9\% emitted past scrublen during steady sinteperiod clain py $6-7<20 \%$ with urea

Steaty state period $1 / 19 / 95 \rightarrow 1 / 2,155$

slury Anolysis $5-2$ aug s.s feed $=1.55 \mathrm{l} / \mathrm{min}$ - but density unrelicele

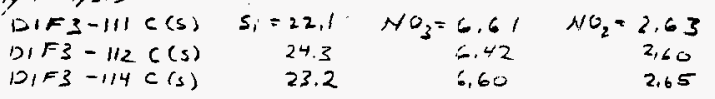

Glups Amalysis, $25 \mathrm{O}_{2} 41.4$ (avg of 8)

Glase Produced $1800 \mathrm{~kg} / \mathrm{day}(\mathrm{pg} \mathrm{5-2)}$

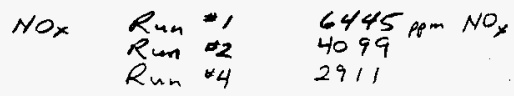

Run 3 likely a pollem

Take Flowe $90 \mathrm{SCFM} S+d=60^{\circ} \mathrm{F}$

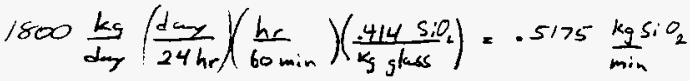

slury sumples:

$$
\begin{aligned}
& \text { IIIC(S) }\left[6.6 / 9 \mathrm{NO}_{3}\left(\frac{\mathrm{mol} N}{14+48 \mathrm{~g}}\right)+2.63 \mathrm{NO}_{2}\left(\frac{\mathrm{mlN}}{14+32}\right)\right] / 22.1 \mathrm{~g} 5 \mathrm{OO}_{2}=7.41 \times 10^{-3} \\
& 112 \text { (s) }\left[6.42\left(\frac{m o l}{62}\right)+2.60\left(\frac{6-1}{46}\right)\right] / 24.3 \\
& =6.59 \times 10^{-3}
\end{aligned}
$$

or $7,02 \frac{\mathrm{molH}}{\mathrm{Kg} \mathrm{SiO}_{2}}$

Source rate $=.5175 \frac{\mathrm{kg} s l_{2}}{\mathrm{~min}} 7.02 \frac{\mathrm{mdN}}{\mathrm{kg} 5 \mathrm{O}_{2}}=3.64$ mol/min

oftyas

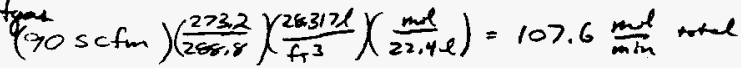

DOT MOMABIE COPY

Tuo Duratek NOX Yield

Project

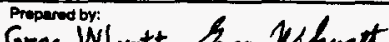

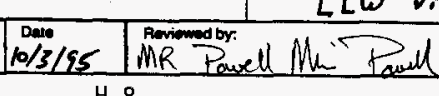

$\frac{0.04 / 3 / 45}{10 / 4}$ 
WHC-ST-W/M-ER-517

Revision 0

\section{* Battelle}

Pacific Northwest Laboratories

\section{CALCIULATION WORKSHEET}

Calc. No. $1+1.5$

$\left[107.6 \min \left(\frac{6445}{10^{6}}\right) / 3.64\right] \times 100=19.1$

$\left[107.6 \frac{\mathrm{mel}}{\min }\left(\frac{4099}{10^{6}}\right) / 3.64 \quad 12.1\right.$

$\left[107.6 \frac{\operatorname{mel}}{\min }\left(\frac{2911}{10^{6}}\right) / 3.64 \quad 7 \times 100 \quad 8.6\right.$

$$
A r g=13.3 \text { yield }
$$


Revision 0

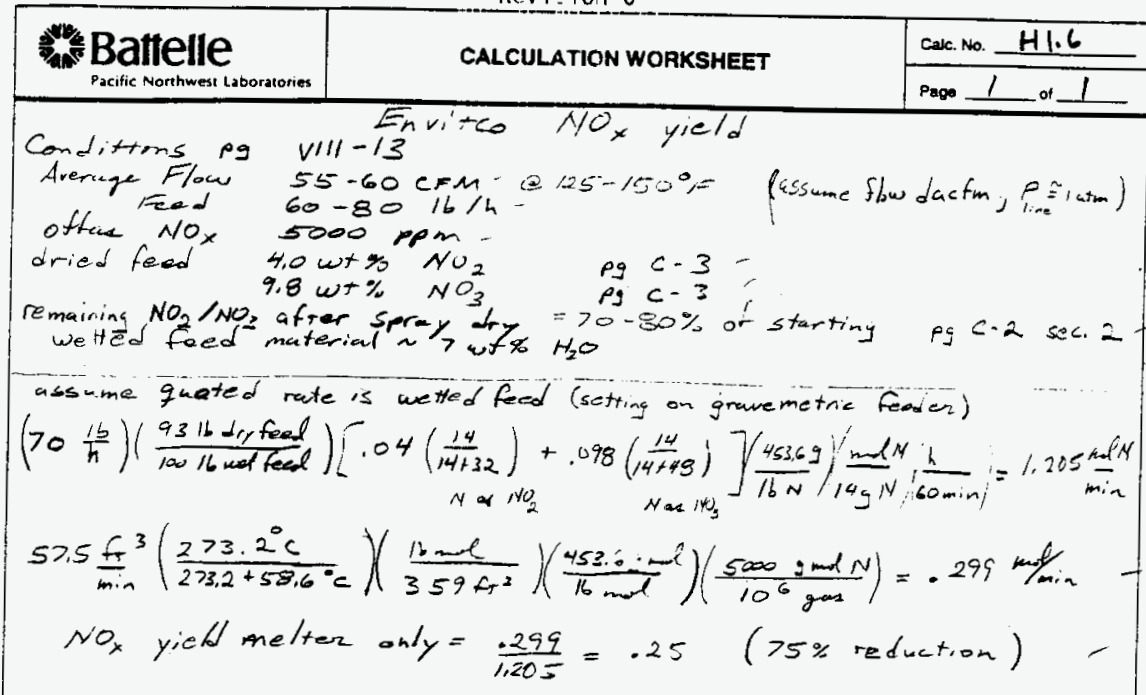

However, 20-30\% hay he evolved in spray dry operation using $25 \%$ evolved in spray dry: overall Mo $x$ yield' is $25-50 \%$

depending on behavior daring spray dry

Th eke value as hid point of range $25 \longleftrightarrow 25+25$

or $37.5 \%$

RET MU ABLE COPY

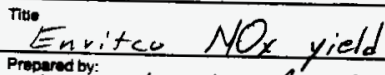

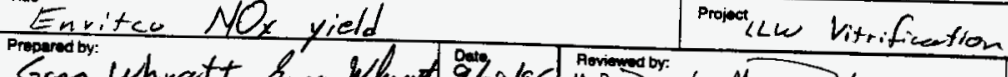

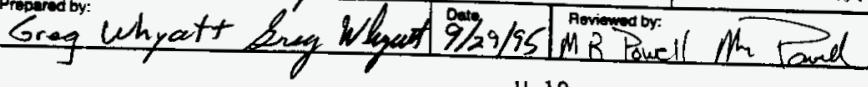

$100 \% 345$

H-10

A1007-115 tan 
Revision 0

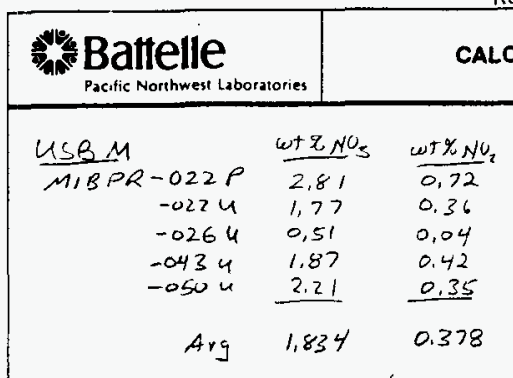

Source to melton $=\left(750 \frac{16}{\mathrm{~h}}\right.$ feed $\left(\frac{\mathrm{h}}{60 \mathrm{~min}}\right)\left(\frac{453.69}{16}\right)\left[.01834\left(\frac{\mathrm{mol}}{14+48}\right)+.00378\left(\frac{\mathrm{mol}}{14+32}\right)\right]$$$
=2,14 \mathrm{molm} / \mathrm{m}
$$

From H1.3 gaseous $\mathrm{NO}_{Y}=6.83 \times 10^{-\mathrm{H}} \mathrm{mol/min}$, Raw feed $=9.49 \mathrm{mot/min}$

$\therefore$ Fad reaction destroys $(9,49-2,14) / 9,49=27.5 \%$ of $\mathrm{NH}_{3} / \mathrm{NO}_{2}$

of gases released, $9.6 \%$ is as $\mathrm{MO}_{\mathrm{X}}$

Residue in feal is destroyed with $\frac{2.14-6.83 \times 10^{-4}}{2,14}=99.97 \%$ efficiency

Prove=- Overall yield $=\left(9.6 * N \mathrm{NO}_{*}\right)(.775)=7.4 \%$

Envitco

$$
\text { NO x yield from melter }=\frac{.299 \mathrm{wr} / \mathrm{m}}{1.205 \frac{\mathrm{moln}}{\mathrm{min}}(.25)}=33 \%
$$

WO, yield from spay dry is unknown so overall process yield is $\geqslant 33 \%(.75)=25 \%$ or minimum $25 \%$

If spray dry was all MOp would be $25+25=50 \%$

BET PAYABLE COPY

Trio secosome

Tito
US BM $/$ Separate
Prepared by:

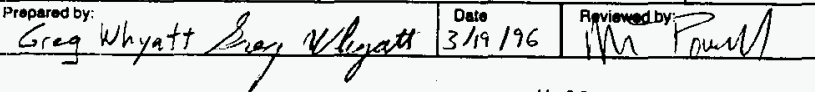

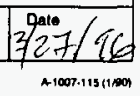

H-11 


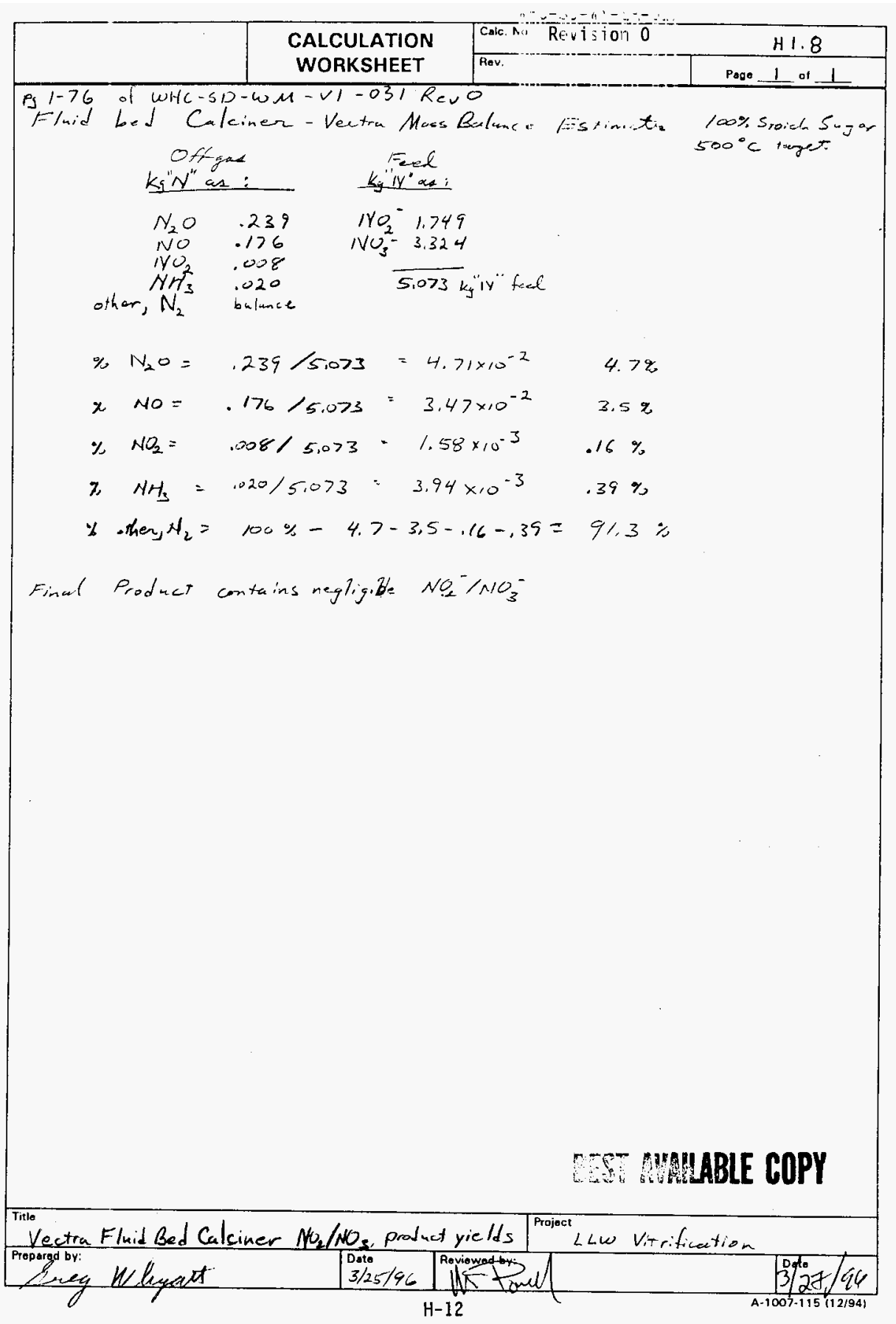


WHC-SD-WM-ER-517

Battelle

Revision 0
CALCULATICN WORKSHEET

$\frac{\text { Calc No. }-H 1,9}{\text { Page } 1 \text { of } 1}$

Duratel 95 -41 of Report

$\mathrm{NH}_{3}$ e $1934 \mathrm{mg} / \mathrm{m}^{2}$ in $99 \mathrm{dsefm}$, H1.5; N source $=3.64 \mathrm{~mm} / \mathrm{min}$

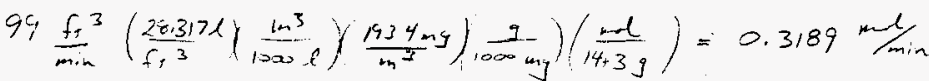

ighore urea - $\mathrm{NH}_{3}$ yield busod on $\mathrm{NO}_{2}^{-} \mathrm{NO}_{3}^{-}=\frac{.3189}{3.64}=8.8 \%$

USBM see 11.3 feed $=9.49$ and l\%inin, Lub duta

$\mathrm{NH}_{3}$ yield on feed prep $=\frac{23.5}{(23+2(18.8)+2(78.2)+23.5)}=9.8 \%$

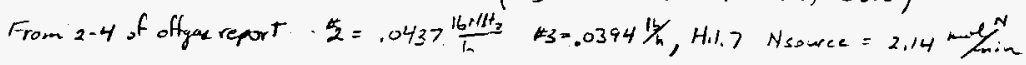

$$
\begin{gathered}
\left(\frac{.0437+.0394}{2} \frac{\mathrm{hl}}{\mathrm{hr}}\right)\left(\frac{453,6 \mathrm{~g}}{16}\right)\left(\frac{\mathrm{ml}}{17 \mathrm{~g}}\right)\left(\frac{\mathrm{h}}{60 \mathrm{~min}}\right)=1.85 \times 10^{-2} \mathrm{~mol} / \mathrm{min} \mathrm{NH}_{3} \\
\text { Melten } \mathrm{NHH}_{3} \text { yidd } \cong \frac{1.85 \times 10^{-2}}{2.14}=0.87 \%
\end{gathered}
$$

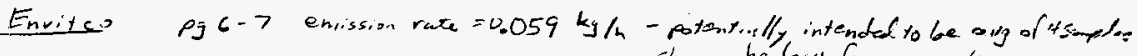

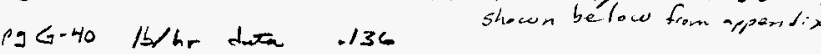

.124

.0715

$\frac{.0715}{A_{\mathrm{rg}}}=.1121 \mathrm{l} / \mathrm{hr} \quad .0509 \mathrm{~kg} / \mathrm{hr} \mathrm{HH}_{3}$

From 11.16 source to me $/ \mathrm{ten}=1.205$ wot $\mathrm{V} / \mathrm{min}$

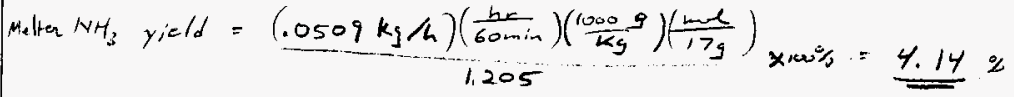

WOAUABL COPY

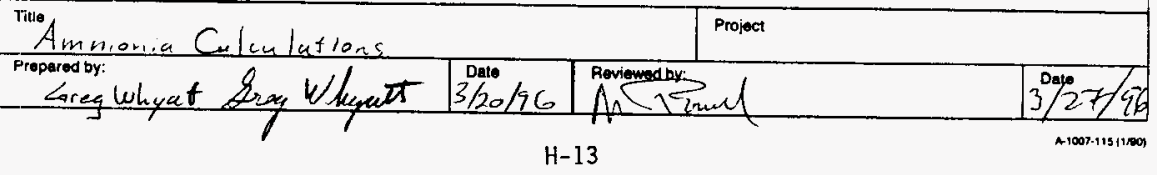


Estriate volutility of Iotine bajed on neutrom areyiation anclysis

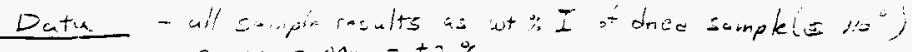

$$
\begin{aligned}
& \text { aneracey } \varrho 1000 \mathrm{HPm}= \pm 2 \% \\
& \text { e } 100 \mathrm{ppm}= \pm 10 \mathrm{t}
\end{aligned}
$$

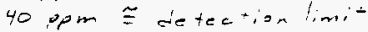

\begin{tabular}{|c|c|c|c|c|c|}
\hline & & $w+\% I$ & $A L_{2} O_{=}$ & $\mathrm{S}_{1} \mathrm{O}_{2}$ & $\mathrm{SaO}$ \\
\hline$B I F>$ & 00241. & .0904 & 8.12 & 43.8 & 3,85 \\
\hline & 00542 & .1082 & 7.87 & 42,5 & 2.68 \\
\hline & $\infty \& u ?$ & .0941 & 7.92 & 42.4 & 3.79 \\
\hline & 0134 & .1082 & 2,85 & $\dot{y}=3$ & 3.74 \\
\hline 3169 & 00241 & .0156 & 13.2 & 59.2 & 5.35 \\
\hline & 00542 & .0105 & 12.0 & 63.2 & 5.20 \\
\hline & $006 u$ & $.00 \% 5$ & 14,5 & 60.3 & 5.72 \\
\hline & $\begin{array}{l}00843 \\
01345\end{array}$ & $\begin{array}{l}.025 \\
.0075\end{array}$ & $\begin{array}{l}15.1 \\
13.1\end{array}$ & $\begin{array}{l}64,1 \\
63.0\end{array}$ & $\begin{array}{l}5.57 \\
5.61\end{array}$ \\
\hline
\end{tabular}

Bcosese + Wikox

\begin{tabular}{|c|c|c|c|c|c|}
\hline$\omega \subseteq T \subseteq$ & $w+6 E$ & $\mathrm{Al}_{2} \mathrm{O}_{3}$ & $\mathrm{C.O}$ & $I_{\text {in }}=$ & \\
\hline$w 1 F 12-40744$ & $.0978\left(\begin{array}{c}A r y \\
2 \\
2\end{array}\right)$ & 14.9 & 3.76 & $12 / 3$ is:- & $\operatorname{loc}_{4}$ \\
\hline wi $\mathrm{Go}^{7}-425: 14$ & $<.0025$ & 19.1 & 4.06 & $=18 \quad 15: 00$ & \\
\hline
\end{tabular}

\section{USBM}

$M 1$

$$
\begin{array}{rr}
A P R-\infty & .1093 \\
A R K-0024 & .1206 \\
B P R-0224 & .1129 \\
-0264 & .1247 \\
0434 & .1095 \\
0504 & .1117
\end{array}
$$

$\begin{array}{rr}M 161-0024 & .0118 \\ M 161-0.44 & .0081 \\ 6054 & .0061 \\ 0884 & .0032 \\ 0104 & .0076 \\ 0114 & <.0014 \\ 0124 & .0053\end{array}$

$\begin{array}{lll}\frac{5.0_{2}}{47.1} & & \text { tions } \\ 41 / 10 & 8: 30 \\ 49.7 & 2110 & 10: 0 \\ 50.1 & 1 / 27 & 8: 40 \\ 51.3 & 1127 & 1620 \\ 50.3 & 1 / 31 & 1120 \\ 50.2 & 211 & 0: 15\end{array}$

59,4

58,5

58.8

54.6

55.7

57.2

58.4
Correspondence betwec-

feed Sumpte 0 in meiter ferd t,.elc not deterininail. Use awerage feed Valmes.

\section{a.M HOSE COPY}

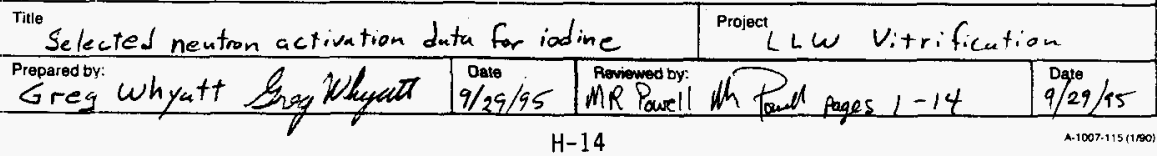




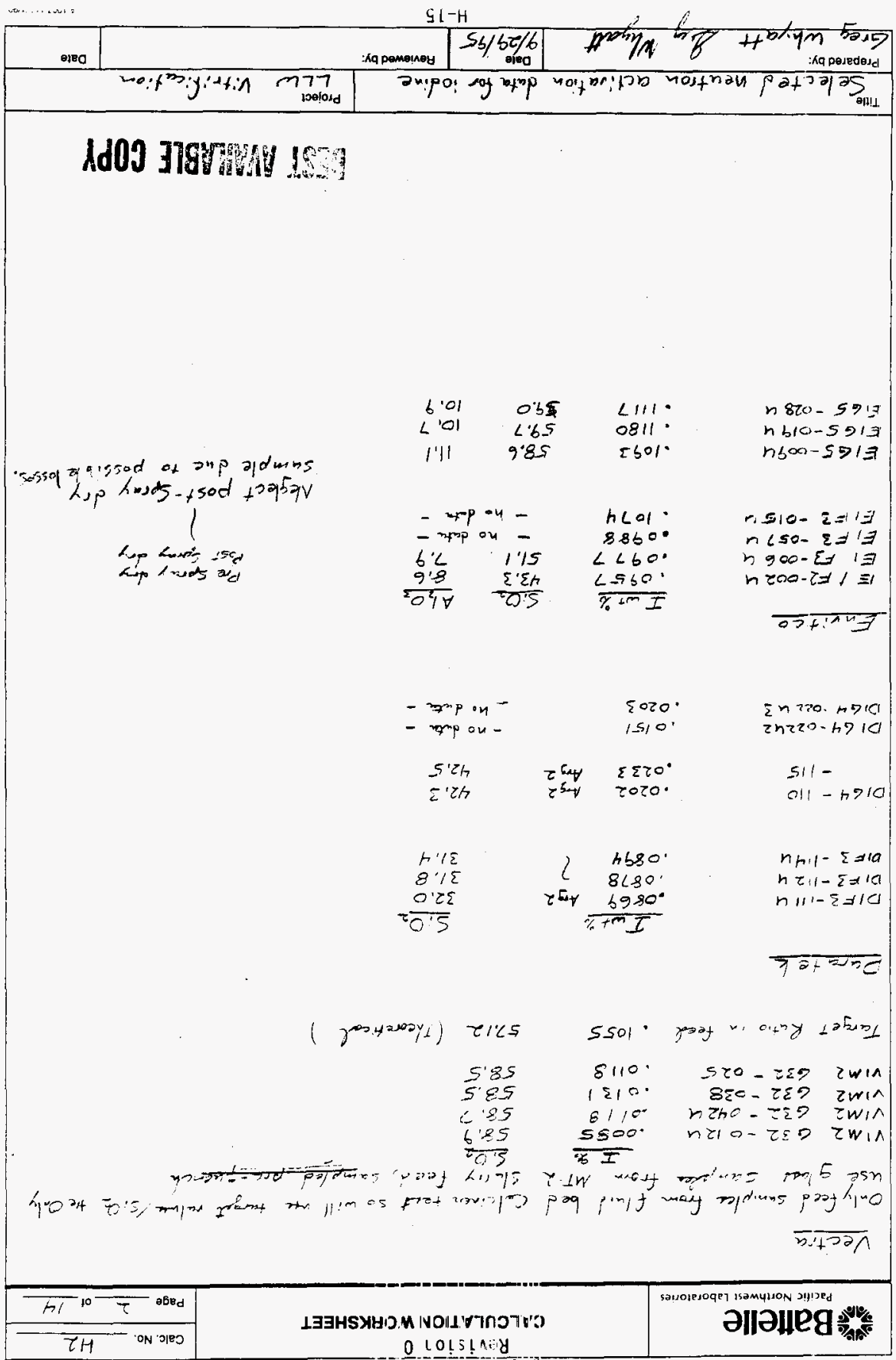


$B \& W$

\begin{tabular}{|c|c|c|c|c|c|c|c|c|c|c|c|c|}
\hline Greg AV & $28 / 95$ & $6: 47 \mathrm{pm}$ & $10 D 1$ & INE VC & DLATILITY & $\overline{\text { ESTIMA }}$ & ATES & & & & & \\
\hline & & & & & & & & & & & & \\
\hline BABCOCK & D WILCO & & & & & & & & & & & \\
\hline Feed Infor & & & & & Glass Infor & mation & & & $\mathrm{Al} 2 \mathrm{O} 3$ & $\mathrm{SiO} 2$ & $\mathrm{CaO}$ & \\
\hline (samples & $-X X X X)$ & & & & (samples & $31 \mathrm{G} 9-\mathrm{X}$ & $x(x)$ & & Tie Calc & Tie Calc & Tie Calc & \\
\hline sample \# & $w t \%$ & $w t \%$ & $w t \%$ & $w t \%$ & $w t \%$ & $w t \%$ & $w t \%$ & $w t \%$ & percent & percent & percent & Average \\
\hline B1F7- & 1 & $\mathrm{Al} 2 \mathrm{O} 3$ & $\mathrm{SiO} 2$ & $\mathrm{CaO}$ & 1 & $\mathrm{Al} 2 \mathrm{O} 3$ & $\mathrm{SiO} 2$ & $\mathrm{CaO}$ & volatile & volatile & volatile & Volatility \\
\hline $002 \cup 1$ & 0.0904 & 8.12 & 43.8 & 3.85 & 0.0156 & 13.2 & 59.2 & 5.35 & $89 . \overline{38455}$ & 87.23242 & 87.58167 & 88.06622 \\
\hline 005u2 & 0.1082 & 7.87 & 42.5 & 3.68 & 0.0105 & 12 & 63.2 & 5.2 & 93.63563 & 93.4742 & 93.13238 & 93.41407 \\
\hline 00843 & 0.0941 & 7.92 & 42.4 & 3.79 & 0.0025 & 15.1 & 64.1 & 5.57 & 98.60653 & 98.24265 & 98.19227 & 98.34715 \\
\hline $013 \mathrm{U}$ & 0.1082 & 7.85 & 42.3 & 3.74 & 0.0075 & 13.1 & 63 & 5.61 & 95.84633 & 95.34592 & 95.37893 & 95.52372 \\
\hline & & & & & & & & & & & & \\
\hline & & & & & & & & & & Overall Ave & rage & 93.83779 \\
\hline
\end{tabular}

站 
WSTC

\begin{tabular}{|l|l|l|l|l|l|l|l|l|l|l|}
\hline WESTINGHOUSE SCIENCE AND TECHNOLOGY CENTER IODINE VOLATILITY CALCULATION \\
\hline
\end{tabular}

$\stackrel{ }{ \pm}$ 
USBM

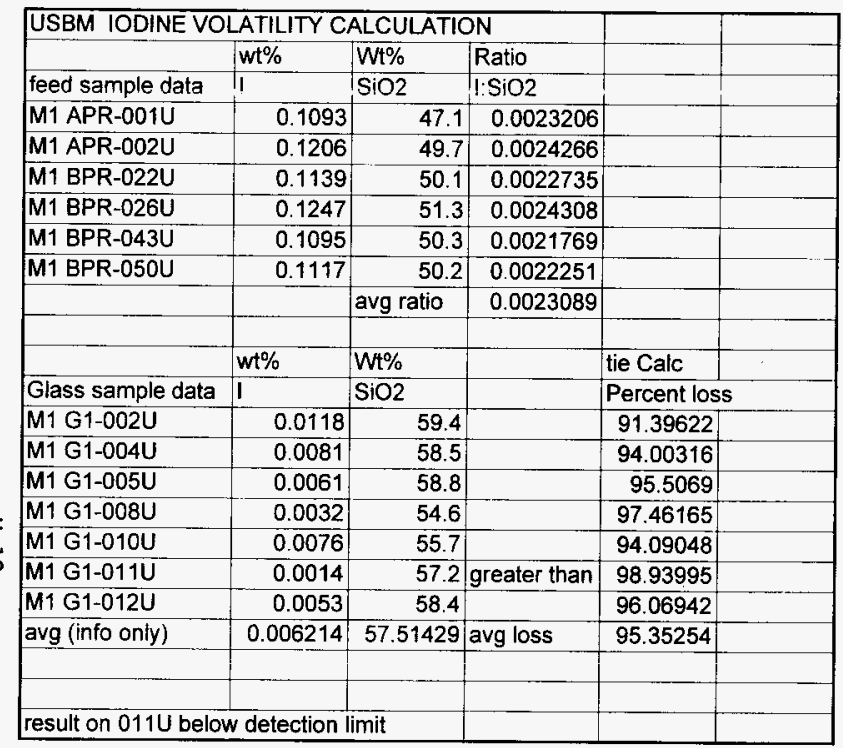


Vectra

\begin{tabular}{|c|c|c|c|c|c|c|}
\hline \multicolumn{7}{|c|}{ VECTRA IODINE VOLATILITY CALCULATION } \\
\hline & & & SiO2 tie & SrO glass & SrO tie & \\
\hline Glass Samples & $w t \%$ & $\mathrm{SiO} 2 \mathrm{wt} \%$ & $\%$ loss & $w t \%$ & $\%$ loss & \\
\hline V1M2 G32-012u & 0.0085 & 58.9 & 93.23543 & 0.051 & 86.38571 & \\
\hline V1M2 G32-042u & 0.0118 & 58.7 & 90.57719 & 0.059 & 83.66285 & \\
\hline V1M2 G32-038u & 0.0131 & 58.5 & 89.50332 & 0.055 & 80.54394 & \\
\hline \multirow[t]{2}{*}{ V1M2 G32-025u } & 0.0118 & 58.5 & 90.54498 & 0.055 & 82.4747 & \\
\hline & & average & 90.96523 & & 83.2668 & \\
\hline \multirow{2}{*}{\multicolumn{7}{|c|}{$\begin{array}{l}\text { Feed data was not available for slurry feed } \\
\text { therefore based on target recipie pg } 3-76,78\end{array}$}} \\
\hline & & & & & & \\
\hline \multirow{4}{*}{ Target Feed } & & & & & & \\
\hline & $i$ & $\mathrm{SiO} 2$ & $\mathrm{SrO}$ & & & \\
\hline & 0.1171 & 54.89 & 0.095654 & & & \\
\hline & & & & & & \\
\hline \multirow{2}{*}{\multicolumn{7}{|c|}{ Note: In this case, SrO tie result is preferred due to possible non-steady state concentrations }} \\
\hline & & \multicolumn{4}{|c|}{ of SrO and I because these components were not in the startup glass. } & \\
\hline
\end{tabular}




\begin{tabular}{|c|c|c|c|c|c|}
\hline DURATEK IC & DINE VOLA & ATILTY CAL & CULATION & & \\
\hline & & & & & \\
\hline & & & Ratio & & \\
\hline feed & $w t \%$ & $\mathrm{SiO} 2 \mathrm{wt} \%$ & I:SiO2 & & \\
\hline D1 F3-111U & 0.0869 & 32 & 0.002716 & & \\
\hline D1 F3-112U & 0.0878 & 31.8 & 0.002761 & & \\
\hline D1 F3-114U & $0 . \overline{0894}$ & 31.4 & 0.002847 & $\mathrm{SiO} 2 \mathrm{Tie} \mathrm{Calc}$ & \\
\hline & & & & Percent Loss= & 81.5148 \\
\hline & & avg & 0.002775 & & \\
\hline D1 G4-110 & 0.0202 & & & & \\
\hline D1 G4-115 & 0.0233 & 42.5 & 0.000548 & & \\
\hline & & & & & \\
\hline & & avg & 0.000513 & & \\
\hline
\end{tabular}

\begin{tabular}{l}
$T$ \\
\multirow{1}{0}{}
\end{tabular} 


\section{Envitco}

\begin{tabular}{|c|c|c|c|c|c|c|c|}
\hline \multicolumn{8}{|c|}{ ENVITCO IODINE VOLATILITY } \\
\hline & & & & ratio & ratio & & \\
\hline feed sample & $1 \mathrm{wt} \%$ & SiD2 $w t \%$ & Al $203 \mathrm{wt} \%$ & !:SiO2 & 1:Al2O3 & & \\
\hline \multirow[t]{4}{*}{ E1 F2-002U } & 0.0957 & 43.3 & 8.6 & 0.00221 & 0.0111279 & & \\
\hline & & & & & & & \\
\hline & & & & & & & \\
\hline & & & & ratio & ratio & $\mathrm{SiO} 2 \mathrm{Tie}$ & $\mathrm{Al} 2 \mathrm{O} 3 \mathrm{Tie}$ \\
\hline Glass Samples & $1 \mathrm{wt} \%$ & $\mathrm{SiO} 2 \mathrm{w} t \%$ & $\mathrm{Al} 2 \mathrm{O} 3 \mathrm{wt} \%$ & $1: \mathrm{SiO} 2$ & I:AL2O3 & $\%$ loss & $\%$ loss \\
\hline E1 G5-009u & 0.1093 & 58.6 & 11.1 & 0.001865 & 0.0098468 & 15.60854 & 11.51214 \\
\hline E1 G5-019u & 0.118 & 59.7 & 10.7 & 0.001977 & 0.011028 & 10.56992 & 0.89747 \\
\hline \multirow[t]{5}{*}{ E1 G5-028u } & 0.1117 & 59 & 10.9 & 0.001893 & 0.0102477 & 14.34019 & 7.909848 \\
\hline & & & & & & & \\
\hline & & & & & avg & 13.50621 & 6.773152 \\
\hline & & & & & & & \\
\hline & & & & \multicolumn{2}{|c|}{ overall average \% loss } & $10 . \overline{13968}$ & \\
\hline
\end{tabular}

$\underset{1}{ \pm}$ 
MAY. OQ-95 TUE 16:43 BR OF GEOCHEMISTRY

FAX NO. 83032363200

\section{U.S. GEOLOGICAL SURVEY \\ BRANCH OF GEOCHEMMSTRY BOX 25046, DFC, M/S 973 Denver, Colorado 80225-0046}

DATE: $5 / 9 / 95$

TO: ERLL MAST

FAX\#: (509) $372-2.810$

FROM: PauL Lamothe

BGC FAX\#: (303)236-3200

\section{COMMENTS:}

Here is AU THE IODIUE DATA

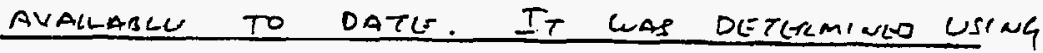

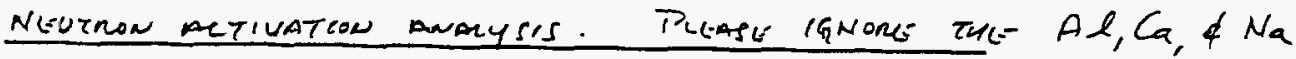

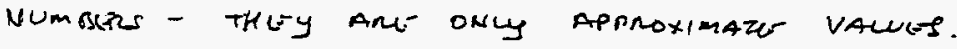

COVER SHEET \& 1 PAGES

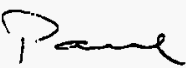


JOB HO: WDE9

SUBMITTER: =AMOTHE, PAUL

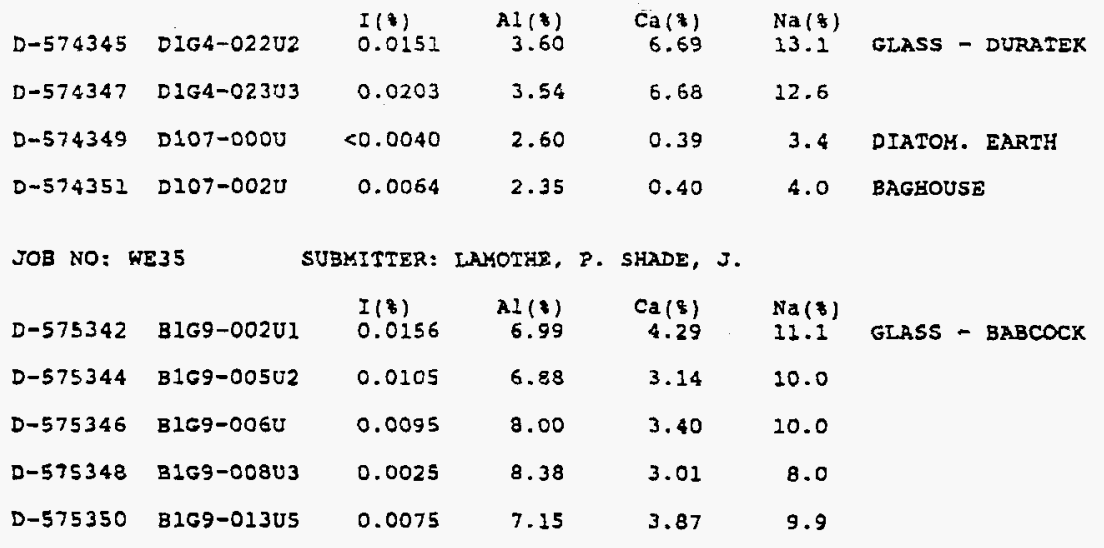

JOB NO: WFO1

$\begin{array}{ll}D-576639 & E 1 G 5-009 U \\ D-576641 & E 1 G 5-019 U \\ D-576643 & E 1 G 5-028 U\end{array}$

JOB NO:

D-577402 BIF7002U

D-577403 BIF7005U2

D-577404 BIE7008U3

D-577405 BIF70130

D-577406 EIF2002U

D-577407 EIF3006U
SUBMITIER: LAMOTHE, PAUL
$I(t)$
0.1093
AI (3)
Ca(z)
0.19
$\mathrm{Na}(\mathrm{z})$
GLASS - ENVICTO
0.1180
5.95
0.25
12.6
0.1117
5.98
0.24
12.7

SUBMITTER: WILSON, STEVE LAMOTHE, PAUL

$\begin{array}{lrrrr}I(\$) & A L(8) & C a(8) & \text { Na(z) } \\ 0.0904 & 4.47 & 2.73 & 9.7 & \text { FEED - BABCOCK } \\ 0.1082 & 4.33 & 2.71 & 10.3 & \\ 0.0941 & 4.24 & 3.00 & 9.8 & \\ 0.1082 & 4.40 & 2.89 & 10.2 & \\ 0.0957 & 4.54 & <0.05 & 9.7 & \text { FEED - ENVICTO } \\ 0.0977 & 4.21 & <0.04 & 8.3 & \end{array}$


JOB NO: WF65 SIUBHITTER: LAMOTHE, PAUI,

\begin{tabular}{|c|c|c|c|c|c|c|}
\hline-577817 & H1G07-425U4 & $\begin{array}{c}=(8) \\
<0.0025\end{array}$ & $\begin{array}{l}\text { Al (5) } \\
10.4\end{array}$ & $\begin{array}{r}\mathrm{Ca}(\$) \\
3.35\end{array}$ & $\begin{array}{l}\mathrm{Na}(3) \\
15.4\end{array}$ & GIASS - WSTC \\
\hline & W1A13-406U4 & $<0.0030$ & 9.69 & 4.10 & 0.21 & IE \\
\hline
\end{tabular}

JOB NO: พH58

SUBHITTER: WILSON, STEVE

\begin{tabular}{|c|c|c|c|c|c|c|}
\hline$D-583266$ & MIAPR-0OIU & $\begin{array}{l}=(8) \\
0.1093\end{array}$ & $\begin{array}{r}\text { AL ( }(2) \\
4.97\end{array}$ & $\begin{array}{r}C a(3) \\
3.51\end{array}$ & $\begin{array}{l}\mathrm{Na}(8) \\
12.4\end{array}$ & $\operatorname{FIED}-$ BOK (USBM) \\
\hline$D-583268$ & MIAPR-002U & 0.1206 & 5.19 & 3.16 & 23.9 & \\
\hline$D-583270$ & HLBPR-022U & 0.1139 & 4.99 & 3.42 & 12.6 & \\
\hline$D-583272$ & HIBPR-026U & 0.1247 & 5.21 & 3.24 & 13.2 & \\
\hline$D-583274$ & MLBPR $-043 U$ & 0.1095 & 5.06 & 3.42 & 12.5 & \\
\hline D-583276 & MIBPR-OSOU & 0.1117 & 4.99 & 3.40 & 11.8 & \\
\hline$D-583278$ & $M 1 C 4-001 U$ & 0.7201 & 0.35 & 1.10 & 22.9 & CYCLONE - USBY \\
\hline$D-583280$ & H1BS-001U & 1.0350 & 0.18 & 0.26 & 23.2 & BAGHOUSE - USBM \\
\hline
\end{tabular}

JOB NO: WH81

SUBMITTER: SHADE, J. WIESON, STEVE

\begin{tabular}{|c|c|c|c|c|c|c|}
\hline$D-583636$ & V1PP1I21-015 & $\begin{array}{l}I(z) \\
0.0797\end{array}$ & $\begin{array}{r}A 1(8) \\
4.81\end{array}$ & $\begin{array}{r}\mathrm{Ca}(8) \\
2.30\end{array}$ & $\begin{array}{l}\mathrm{Na}(8) \\
10.4\end{array}$ & FEED - VECTRA \\
\hline$D-583638$ & V1FPII21-016 & 0.0866 & 5.04 & 1.98 & 10.6 & \\
\hline
\end{tabular}

JOB NO: WH99 SUBKITTER: IAMOTHE, PAUL SHADE J.

\begin{tabular}{|c|c|c|c|c|c|c|}
\hline$D-583957$ & VLK2/G320120 & $\begin{array}{l}I(8) \\
0.0085\end{array}$ & $\begin{array}{r}A 1(8) \\
5.08\end{array}$ & $\begin{array}{r}\text { Ce }(8) \\
2.17\end{array}$ & $\begin{array}{l}\mathrm{Na}(8) \\
11.3\end{array}$ & GLASS - VECTRA \\
\hline D-583959 & V1M2/G34-016 & 0.0115 & 5.39 & 2.28 & 11.4 & \\
\hline D-58396I & $v_{1 K 2 / G 32042 U}$ & 0.0118 & 5.37 & 2.25 & 11.7 & \\
\hline$D-583963$ & VIK3/G34-072 & 0.0197 & 5.31 & 2.24 & 21.7 & \\
\hline$D-583965$ & $v 1 K 3 / 632076 U$ & 0.0202 & 5.35 & 2.02 & 12.3 & \\
\hline$D-583967$ & $V 1 M 4 / G 32090 U$ & 0.0189 & 5.32 & 2.06 & 12.4 & \\
\hline$D-583969$ & V1H4/G32098U & 0.0255 & 5.32 & 2.09 & 12.2 & \\
\hline$D-583971$ & v1M3/G32060u & 0.0176 & 5.54 & 1.95 & 12.3 & \\
\hline
\end{tabular}

JOB NO: HJOl SUBMITIER: LAMOTHE PAUL SHADE, J

\begin{tabular}{|c|c|c|c|c|c|c|}
\hline D-583975 & v142/632-038 & $\begin{array}{l}I(5) \\
0.0131\end{array}$ & $\begin{array}{r}A 1(3) \\
5.05\end{array}$ & $\begin{array}{r}\mathrm{Ca}(z) \\
2.24\end{array}$ & $\begin{array}{l}\operatorname{Ma}(3) \\
11.3\end{array}$ & GLASS - VECTRA \\
\hline$D-583977$ & $v_{1} \times 2 / G 32-025$ & 0.0118 & 5.45 & 2.28 & 12.2 & \\
\hline
\end{tabular}


JOB NO: HGO5

$\begin{array}{ll}D-578562 & \text { WIF12-407U4 } \\ D-578563 & \text { W1F } 12-40704\end{array}$

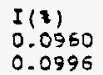

SUBKITTER:
I(I)
$4 \quad 0.0960$
$4 \quad 0.0996$
WILSON, 5. AND LAMOTHE, $P$.

$$
\begin{array}{r}
\text { A1 (8) } \\
8.30
\end{array}
$$$$
\text { 8.21 }
$$

$\mathrm{Na}(8)$
9.74
9.39
FEED - WSTC
JOB NO: WG1O

$\begin{array}{ll}\text { D-578605 } & \text { D1G4-110U7 } \\ \text { D-578606 } & \text { D1G4-110U7 } \\ \text { D-578607 } & \text { D1G4-115U9 } \\ \text { D-578608 } & \text { D1G4-115U9 } \\ & \\ \text { D-578609 } & \text { ARG-1 } \\ & \text { LITERATURE } \\ & \text { ICP (WFO1) }\end{array}$

JOB NO: HG13

$\begin{array}{ll}D-578651 & \text { DIF3-111UA } \\ D-578652 & \text { DIF3-111UB } \\ D-578653 & \text { DIF3-112UA } \\ D-578654 & D 1 F 3-112 U B \\ & \\ D-578655 & D 1 F 3-114 U A \\ D-578656 & \text { DIF3-114UB }\end{array}$

JOB NO: WG49

$\begin{array}{ll}D-583113 & M 1 G 1-0020 \\ D-583115 & M 1 G 1-004 U \\ D-583117 & M 1 G 1-005 U \\ D-583119 & M 1 G 1-008 U \\ D-583121 & \text { MGI-010U } \\ D-583123 & M 1 G 1-011 U \\ D-583125 & \text { MIG1-012U } \\ D-583127 & \text { MIT3-001U }\end{array}$

SUBMITTER: LAYOTHE, RAUL

$\begin{array}{lrrr}I(7) & \text { Al (8) } & \text { Ca(8) } & \text { Na(8) } \\ 0.0193 & 3.55 & 5.59 & 12.25 \\ 0.0211 & 3.57 & 5.55 & 12.07 \\ 0.0224 & 3.63 & 5.10 & 12.17 \\ 0.0241 & 3.52 & 5.55 & 12.06 \\ & & & \\ 0.0032 & 2.69 & 0.60 & 8.08 \\ \text { NA } & 2.47 & 1.01 & 8.31 \\ - & 2.4 & 1.1 & -\end{array}$

\begin{tabular}{|c|c|c|c|c|c|}
\hline $\begin{array}{l}I(1) \\
0.0895 \\
0.0843\end{array}$ & $\begin{array}{r}A 1(8) \\
2.65 \\
2.61\end{array}$ & $\begin{array}{r}\mathrm{Ca}(3) \\
3.93 \\
4.06\end{array}$ & $\begin{array}{r}\text { Na (3) } \\
9.19 \\
9.20\end{array}$ & EEED & - DURATLR \\
\hline $\begin{array}{l}0.0876 \\
0.0880\end{array}$ & $\begin{array}{l}2.59 \\
2.51\end{array}$ & $\begin{array}{l}4.38 \\
3.63\end{array}$ & $\begin{array}{l}9.25 \\
8.94\end{array}$ & FEED & '. \\
\hline $\begin{array}{l}.0895 \\
0.0893\end{array}$ & $\begin{array}{l}2.60 \\
2.60\end{array}$ & $\begin{array}{l}3.48 \\
3.73\end{array}$ & $\begin{array}{l}9.13 \\
8.95\end{array}$ & FEED & 1. \\
\hline
\end{tabular}

SUBMITTER: WILSON, $S$. AND SHADE

SUBMITTER: LAYOTHE, PAUL

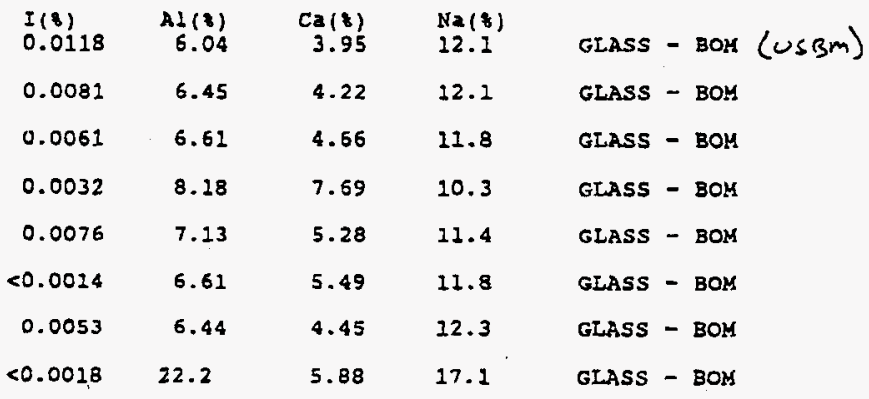


WHIS-SD-WM-ER-E 17

INATA H2 13/14 Revision 0

JOB ND: WG55

D-583220 ELF3-05\%U

D-583222 EIF3-015U
SUBKITTER.: LAMOTHE, PAUL

$\begin{array}{lrlll}I(8) & \text { Al }(8) & C a(8) & \text { Na(8) } & \\ 0.0988 & 4.87 & <0.09 & 10.3 & \text { FEED - ENVICTO } \\ 0.1074 & 6.08 & <0.09 & 13.3 & \text { EEED - ENVICTO }\end{array}$




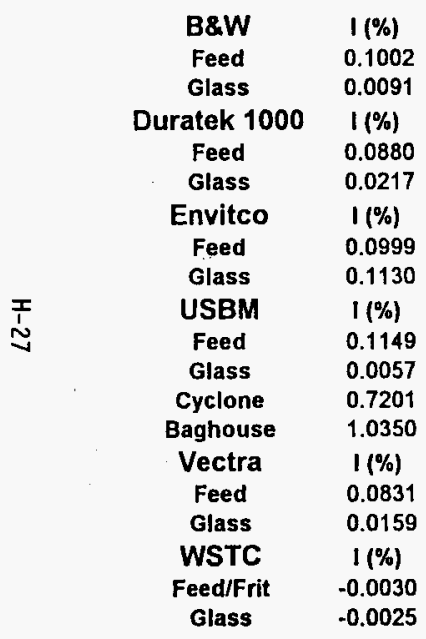
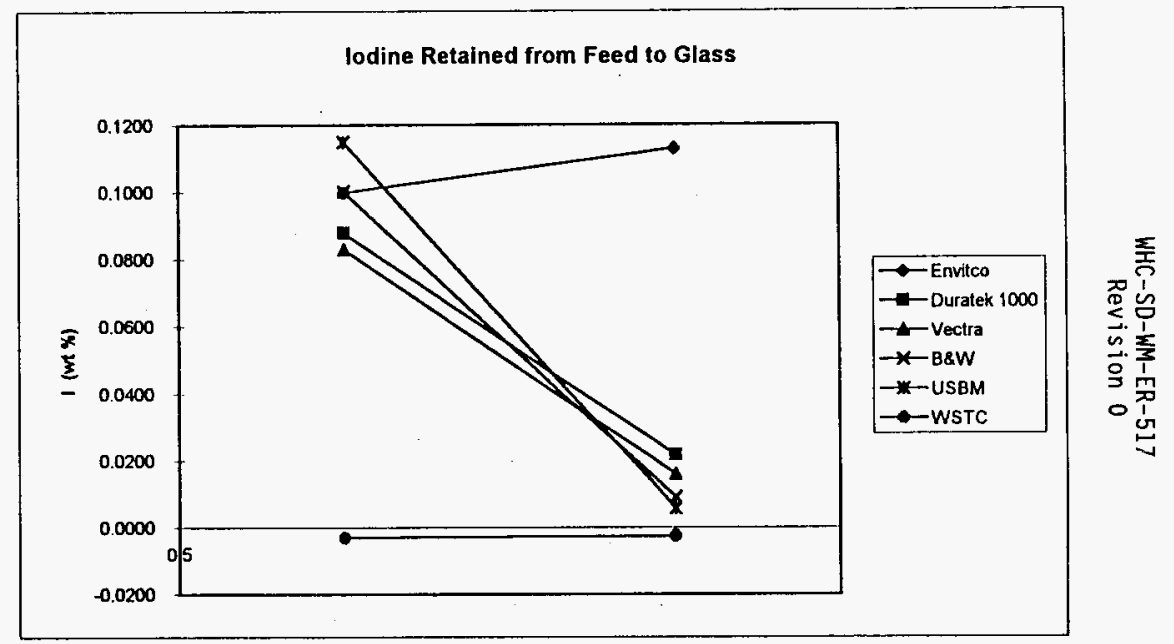


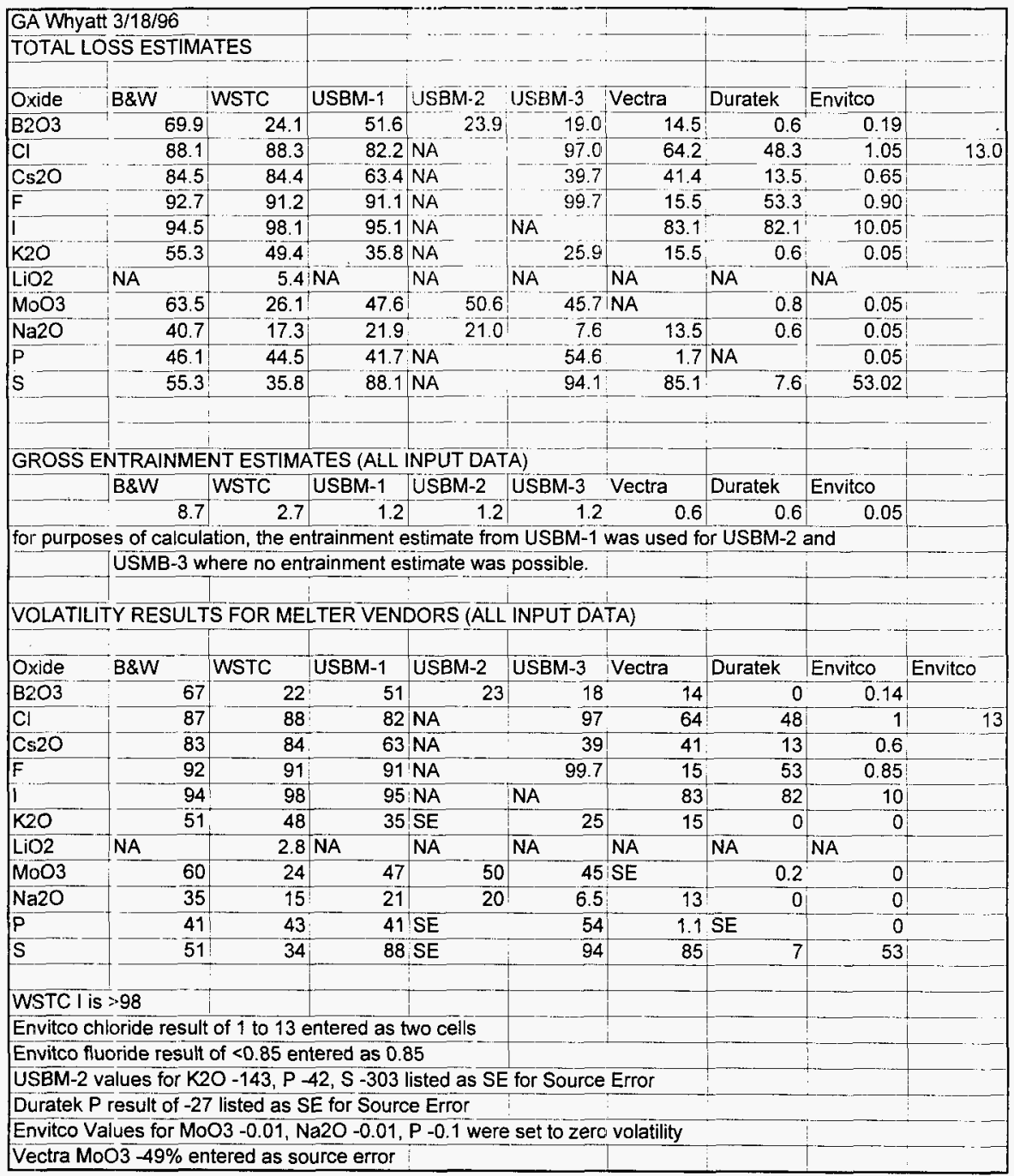


WHC-SD-WM-ER-517

Revision 0

APPENDIX I

REVIEW OF FLUORIDE ANALYTICAL DISCEPANCIES

I-1 
WHC-SD-WM-ER-517

Revision 0

This page intentionally left blank.

I-2 
LETTER REPORT: FLUORIDE ANALYTICAL DISCREPANCIES FOR PHASE- 1 LOW-LEVEL WASTE VENDOR TESTING

\section{C.J. Freeman}

June 1995

Prepared for

The U.S. Department of Energy

Under Contract DE-AC06-76RLO 1830

Pacific Northwest Laboratory

Richland, Washington 99352 


\section{INTRODUCTION}

Analyses of Hanford LoW-Level Waste (LLW) feed and glass constituents from the various vitrification testing vendors were provided from the U.S. Geological Survey (USGS), Corning Incorporated (CELS Laboratory Services), Quanterra, and Pacific Northwest Laboratory (PNL). The feed materials were composed of a standard high sodium LLW simulant (prepared by Optima Chemicals) mixed with glass forming additives which were specified by the vendor. After the first few samples were analyzed it was apparent that the measured fluoride in the feeds differed from the targets for those simulants. As a result, Westinghouse Hanford Company (WHC) requested that PNL investigate this data to ascertain its validity for subsequent mass balance calculations. Possible explanations for the observed fluoride discrepancies in the waste simulant and feed materials were that the fluoride analytical errors were larger than expected, the fluoride was not fully homogenized in the samples (since a significant fraction of the fluoride is present as solid sodium fluoride in the waste simulant), or the fluoride was not added accurately to the simulant to start with.

\section{FLUORIDE ANALYTICAL TECHNIQUES}

From an analytical standpoint fluoride analyses in solid materials can be extremely difficult to obtain. Soluble fluoride can be analyzed quite well with ion Chromatography (IC) or Specific lon Electrode (SIE) techniques. The difficulty with measuring fluoride in solid samples arises when the sample is "fused" (for example, a peroxide/acid fusion) and the fluoride is driven off during heating the sample. Additionally, solid samples with an appreciable degree of silica content can produce silica-fluoride complexes (where silica is present in each of the vendor's glass forming additives) after fusing which can not be quantified with IC or SIE instruments. As a result, fluoride values from fused samples are typically not reported by PNL, only "soluble fluoride" values from IC or SIE are reported as accurate. LLW simulant samples from the original batch make-up tanks, prepared by Optima Chemical, were only analyzed by PNL and Quanterra. Like PNL, Quanterra's fluoride analysis capacity is limited by the techniques/sample preparations just described. As a result, both PNL and Quanterra analytical reports showed 57 to $94 \%$ lower fluoride than the target values for each of the waste simulant batches produced by Optima.

Both USGS and CELS reported having much more developed techniques for fluoride analysis of solid and slurry samples. The following fluoride analysis description was provided by Paul J. Lamothe of USGS. This procedure was used for all of the LLW samples analyzed by USGS. 
Additionally, it is a routine method they have used for the past 15 years for the determination of fluoride in alumino-silicate rocks.

If the material is in a slurry form, 10 grams of the slurry are first added to a $50 \mathrm{~mL}$ glass beaker, dried for 24 hours at $110^{\circ} \mathrm{C}$, followed by an additional 24 hour drying period at $150^{\circ} \mathrm{C}$. The dry residue is then weighed, collected, homogenized, and analyzed for fluoride content using the following method: $200-\mathrm{mg}$ of sample is mixed with 1.2 grams of lithium metaborate $\left(\mathrm{LiBO}_{2}\right)$ in a graphite crucible followed by fusing the mixture in a muffle furnace at $900^{\circ} \mathrm{C}$ for 10 minutes. The molten bead is then poured from the crucible into a polypropylene beaker containing $100 \mathrm{~mL}$ of $4 \%$ nitric acid. After complete dissolution $100 \mathrm{~mL}$ of a buffer solution is added. (The buffer is a solution of diaminocyclohexanetetracetic acid (DCTA) in $40 \% \mathrm{NaOH}$, sodium citrate, and sodium chloride, adjusted to a $\mathrm{pH}$ of 6.85 with $\mathrm{HCl}$ ). The fluoride concentration is then measured by fluoride-selective electrode potentiometry. The results are then reported for the sample as received, i.e. corrected for the weight loss that occurred by drying, etc. Two quality control check samples are analyzed along with each batch of unknowns. These check samples are comprised of solid alumino-silicate standard reference materials of known fluoride composition.

Note that this technique is equivalent to a standard glass fusion which, as previously mentioned, is prone to fluoride volatilization during fusing as well as fluoride-silica complexing. These problems presently drive PNL glass analysts to report such fluoride analyses as "indication only," if at all.

CELS reported using two different fluoride analyses for solid and liquid/slurry samples. For solid analysis (with expected levels $>0.01 \mathrm{wt} \%$ ) a pyrohydrolysis technique is used where the material is ground, mixed with $\mathrm{U}_{3} \mathrm{O}_{8}$, and heated to $1130^{\circ} \mathrm{C}$ in the presence of steam. The fluoride is then distilled as HF, scrubbed and titrated with thorium nitrate or directly analyzed by an ion-selective electrode method. The ion-selective electrode measurements were used for nearly all of the LLW glass samples since the resulting fluoride concentrations were too low for the titration approach. For liquid and dilute slurry samples CELS directly measured fluoride with IC. As a result, these analyses only provide soluble fluoride data. 


\section{WASTE SIMULANT AND MELTER FEED DISCREPANCIES}

Before looking into the various analytical techniques for sources of error it was first decided to inspect the fluoride data from the various melter feeds (Optima waste simulant with glass forming additives) generated by USGS and CELS to see if some to the discrepancies could be attributed to a solid phase separation. If sampling were performed such that the solids were not completely homogeneous, the error in the fluoride data may be directly attributable to this effect.

In order to accurately assess solids inhomogeneity, other known insoluble waste constituents were compared with fluoride. Chromium was chosen as the comparison species because it was present in the original waste simulant and as a precipitation product during simulant make-up. Similar make-up schemes would have resulted in smaller particle sizes compared to many of the bulk glass formers used, such as silica. In order to calculate comparison values of fluoride and chromium, another wastespecific reference ion was selected which was also fairly soluble in the simulant. Using a soluble reference ion enables easy calculations to be made of the fluoride and chromium content without having to rely on the accuracies of each vendor feed target. The reference ion chosen was chloride since chloride analyses are typically quite good and the data from all the waste and feed simulants showed reasonable (close to target) values for this ion.

The calculation made for fluoride, chromium was "percent unaccounted for ion." This calculation was made with the following equation where $X$ refers to either fluoride or chromium:

\% Unaccounted for $X=100\left(1-\frac{\frac{X_{\text {measured }}}{C l_{\text {measured }}}}{\frac{X_{\text {target }}}{C l_{\text {target }}}}\right)$ 
Table 1. Unaccounted for fluoride and unaccounted for chromium from various analyzed LLW melter feeds

\begin{tabular}{|l|c|c|c|c|c|c|c|}
\hline Material: & $\begin{array}{c}\text { B\&W } \\
\text { Feed }\end{array}$ & $\begin{array}{c}\text { B\&W } \\
\text { Feed }\end{array}$ & $\begin{array}{c}\text { Envitco } \\
\text { Feed }\end{array}$ & $\begin{array}{c}\text { WSTC } \\
\text { Feed }\end{array}$ & $\begin{array}{c}\text { Duratek } \\
1000 \\
\text { Feed }\end{array}$ & $\begin{array}{c}\text { Duratek } \\
1000 \\
\text { Feed }\end{array}$ & $\begin{array}{c}\text { SSHTM } \\
\text { Feed }\end{array}$ \\
\hline Analytical Firm: & Corning & USGS & Corning & Corning & Corning & USGS & PNL \\
\hline Unaccounted for.. & & & & & & & \\
& & $86 \%$ & $44 \%$ & $83 \%$ & $54 \%$ & $68 \%$ & $84 \%$ \\
Fluoride & $79 \%$ & $86 \%$ & $15 \%$ & $2 \%$ & $11 \%$ & $17 \%$ & $38 \%$ \\
Standard Dev & $15 \%$ & $25 \%$ & $17 \%$ & & & & \\
Chromium & $52 \%$ & $33 \%$ & $58 \%$ & $19 \%$ & $-12 \%$ & $1 \%$ & $-11 \%$ \\
Standard Dev & $7.7 \%$ & $1.6 \%$ & $17.1 \%$ & $0.9 \%$ & $1.0 \%$ & $0.1 \%$ & $3.4 \%$ \\
\hline
\end{tabular}

The previous equation assumes that the chloride was added on target and completely accounted for upon analysis. The chloride values for the various feeds analyzed appeared close to their target levels. The following table and plot show the results of applying the previous equation to the Babcox \& Wilcox (B\&W), Envitco, Westinghouse Science and Technology Center (WSTC), Duratek, and SSHTM (PNL) LLW melter feeds. Each of these feeds used the same waste simulant compositions. Note that the standard deviation values were calculated using data from repeat analyses.

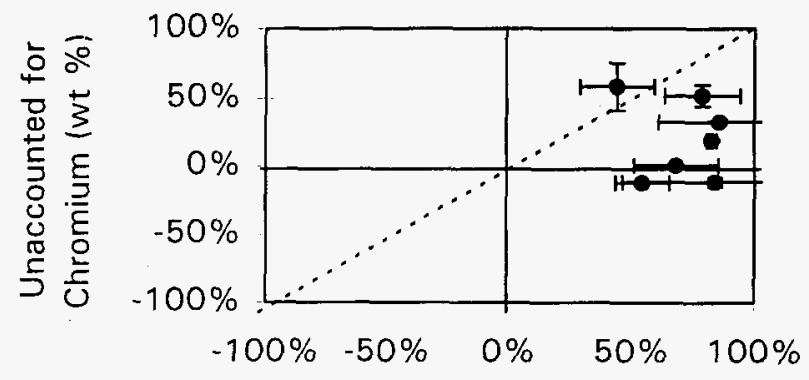

\section{Unaccounted for Fluoride (wt \%)}

Figure 1. Unaccounted for fluoride versus unaccounted for chromium from various analyzed LLW melter feeds

Figure 1 shows the percent of unaccounted for fluoride plotted against unaccounted for chromium. If the variations in all of these species were 
simply due to inhomogeneity of solid constituents, these data would have varied around zero along the $45^{\circ}$ line shown. Instead, only the average chromium data varies around zero, indicating inhomogeneity in some of the samples, and the fluoride values are highly skewed leven within the error ranges shown) to a range of 44 to $86 \%$ unaccounted for fluoride. Note that the Envitco feed was the only sample in this data set which was calcined. The higher degree of chromium than target from the analyses may indicate that this particular sample was slightly richer in waste simulant solids.

Since the previous calculations indicate that the fluoride discrepancies are not largely due to simple sample inhomogeneity the next possibility examined was whether or not the fluoride was accurately added to the waste simulants, at Optima, in the first place. Although strict quality assurance was maintained during these simulant make-ups the possibility of a mismarked container, etc. was hypothesized by several WHC staff. Since the only fluoride analysis performed on the waste simulants were made by PNL and Quanterra, who only analyzed for soluble fluoride, the accuracy of these original analytical checks was poor. Therefore, PNL made a few "control" samples of simulant to cross-check the accuracies of the CELS and USGS fluoride data. These control samples consisted of carefully made individual $250 \mathrm{ml}$ batches of LLW simulant. These samples were then sent to both CELS and USGS for analysis. The results from those analyses are shown in Table 2.

The USGS results, shown in Table 2, accounted for only $17.7 \%$ of the fluoride in the sample. This discrepancy is mostly likely due to the high temperature fusion step used in sample preparation as well as silica-fluoride complexing in such fusions. The CELS results report two sets of numbers. The first set was generated by a simple IC analysis of the sample supernate. As a result these numbers should reflect only the soluble halides in the sample. Indeed, fluoride values from the IC analyses account for only $41 \%$ of that ion. Finally, the last CELS data in Table 2 show the results from their pyrohydrolysis procedure. The fluoride value reported from this procedure accounts for more than the first two, however, it is still low lonly $59 \%$ accounted for). 
Table 2. Fluoride and Chloride Analytical Results for LLW Simulant Control Samples (G1S1-001U \& G1S1-001C)

\begin{tabular}{|c|c|c|c|c|c|c|c|}
\hline Halide & Target & \multicolumn{2}{|c|}{$\begin{array}{c}\text { USGS } \\
\text { (Fusion/IC) }\end{array}$} & \multicolumn{2}{c|}{$\begin{array}{c}\text { CELS } \\
\text { (lon Chrom., } \\
\text { solubles) }\end{array}$} & \multicolumn{2}{c|}{$\begin{array}{c}\text { CELS } \\
\text { (Pyrohydrolysis) }\end{array}$} \\
\cline { 2 - 7 } & $\begin{array}{c}\text { Mass } \\
\text { Content } \\
\text { of Halide }\end{array}$ & $\begin{array}{c}\text { Mass } \\
\text { Content } \\
\text { of Halide }\end{array}$ & $\begin{array}{c}\text { Amount of } \\
\text { Halide } \\
\text { Missing }\end{array}$ & $\begin{array}{c}\text { Mass } \\
\text { Content } \\
\text { of Halide }\end{array}$ & $\begin{array}{c}\text { Amount of } \\
\text { Halide } \\
\text { Missing }\end{array}$ & $\begin{array}{c}\text { Mass } \\
\text { Content } \\
\text { of Halide }\end{array}$ & $\begin{array}{c}\text { Amount of } \\
\text { Halide } \\
\text { Missing }\end{array}$ \\
\hline Chloride & $0.36 \%$ & $0.27 \%$ & $25.8 \%$ & $0.41 \%$ & $-11.3 \%$ & $0.33 \%$ & $9.3 \%$ \\
Fluoride & $0.31 \%$ & $0.05 \%$ & $\mathbf{8 2 . 3} \%$ & $0.13 \%$ & $\mathbf{5 9 . 0 \%}$ & $0.18 \%$ & $41.0 \%$ \\
\hline
\end{tabular}

The ranges of unaccounted for fluoride reported in Table 2 are within the ranges observed for the LLW vendor feeds (see Table 1). Since these same ranges of analytical discrepancy were observed for the control samples as were for each of the vendor feed analyses it is apparent that the fluoride was indeed added near target in the original Optima batches. Furthermore, the target feed values for fluoride are expected to be more accurate for subsequent mass balance calculations than the measured values.

\section{FLUORIDE ANALYSES OF GLASS SAMPLES}

In addition to investigating waste simulant and melter feed fluoride discrepancies WHC also requested looking at the glass analyses to ascertain the validity of this data. The method chosen to present this data was to use an approach similar to the one shown in the previous section, where a reference ion is used to calculate the unaccounted for fluoride based on its target value (i.e. feed composition targets used, not analyzed values). Two reference ions were chosen for this calculation, manganese and molybdenum. However, manganese was expected to provide a better comparison since molybdenum is known to volatilize from high temperature glasses. Nevertheless, sets of calculations for both of these ions are shown in the following table. Note that the standard deviations shown in this table were not generated from duplicate sample analyses but from all of the glass samples analyzed for each test. As a result, this standard deviation is representative of total sample variation and is not equal to the analytical error. However, these standard deviations do encompass the analytical error. 
Table 3. Phase-1 LLW Vendor Testing Glass Fluoride Analyses

\begin{tabular}{|l|c|c|c|c|c|c|c|c|c|c|}
\hline Glass Sample: & B\&W & B\&W & WSTC & WSTC & Duratek & Duratek & Envitco & Envitco & USBM & USBM \\
\hline Analytical Firm: & Corning & USGS & Corning & USGS & Corning & USGS & Corning & USGS & Corning & USGS \\
\hline F/MnO2: & & & & & & & & & \\
Target (waste) & 205.5 & 205.5 & 205.5 & 205.5 & 205.5 & 205.5 & 205.5 & 205.5 & 205.5 & 205.5 \\
Average (glass) & $<0.27$ & 0.73 & 2.62 & 0.95 & 1.10 & 0.74 & 55.86 & $>23.81$ & 2.90 & 2.47 \\
Standard Dev & 0.04 & 0.40 & 1.79 & & 0.25 & 0.29 & 11.02 & 2.90 & 0.64 & 0.45 \\
Unaccounted for F & $>99.9 \%$ & $99.6 \%$ & $98.7 \%$ & $99.5 \%$ & $99.5 \%$ & $99.6 \%$ & $72.8 \%$ & $<88.4 \%$ & $98.6 \%$ & $98.8 \%$ \\
Standard Dev & $0.0 \%$ & $0.2 \%$ & $0.9 \%$ & & $0.1 \%$ & $0.1 \%$ & $5.4 \%$ & $1.4 \%$ & $0.3 \%$ & $0.2 \%$ \\
& & & & & & & & & & \\
F/MoO2: & & & & & & & & & \\
Target (wastel & 205.5 & 205.5 & 205.5 & 205.5 & 205.5 & 205.5 & 205.5 & 205.5 & 205.5 & 205.5 \\
Average (glass) & $<0.09$ & 0.25 & 0.20 & 0.21 & 1.01 & 0.86 & 2.13 & 2.05 & 0.33 & 0.38 \\
Standard Dev & 0.05 & 0.11 & 0.06 & & 0.22 & 0.33 & 0.65 & 0.29 & 0.04 & 0.06 \\
Unaccounted for F & $>100 \%$ & $99.9 \%$ & $99.9 \%$ & $99.9 \%$ & $99.5 \%$ & $99.6 \%$ & $99.0 \%$ & $99.0 \%$ & $99.8 \%$ & $99.8 \%$ \\
Standard Dev & $0.0 \%$ & $0.1 \%$ & $0.0 \%$ & & $0.1 \%$ & $0.2 \%$ & $0.3 \%$ & $0.1 \%$ & $0.0 \%$ & $0.0 \%$ \\
\hline
\end{tabular}

Table 3 shows that the unaccounted for fluoride calculated from both the manganese and molybdenum references are between 98 and $100 \%$ in all cases but the Envitco glasses calculated with a manganese reference ion. Since the molybdenum reference values were suspect due to potential molybdenum volatilization, the manganese referenced values are expected to be the better of the two data sets. The Envitco glass analyzed by USGS shows a less than sign in front of its unaccounted for fluoride value. This was a result of analytical values being less than their detection limits. Since the Corning analyses of the Envitco glass did not run into detection limit problems, it is expected that this is the more accurate of the two values.

Unfortunately, the glass analytical values can not be used to ascertain the analytical errors of the measurement techniques. The few glass fluoride standards available for doing such a calibration have fluoride contents in the range of a few weight percent, far from the expected LLW glasses contents of less that 0.1 weight percent. As a result the analytical errors quantified from the control waste simulant analyses for fluoride are the best known values to apply to the glass analyses for this range of fluoride content.

\section{CONCLUSIONS}

Based on the glass analytical techniques described by both Corning and USGS and the discrepancies found for fluoride in the control samples it is believed that substantial errors could also lie in the glass fluoride analyses provided by each of these firms. It would be recommended that, in addition to using feed target values for fluoride in the mass balance calculations, 
fluoride measured in the off-gas samples aiso be used for those calculations instead of the glass values. This is accomplished by assuming that the portion of the constituent which goes to the glass is equal to feed target amount minus that observed in the off-gas. However, many Phase- 1 vendor tests used off-gas sample filters comprised of silica in the form of quartz or glass. As a result, those samples may also be prone to fluoride analytical problems. Still, this data or the data collected from scrubber and quencher samples (in addition to feed target values for fiuoride) may have the least amount of error for melter mass balance calculations.

Further quantification of USGS and CELS fluoride analytical error may show the reported values to be reasonable for mass balance calculations, however, neither of those firms could explain the analytical discrepancies for the waste simulant control samples. Consequently, it is recommended that PNL's pyrohydrolysis analytical equipment be used to substantiate this data. Previous pyrohydrolysis data, reported by PNL in 1989 (Goles, 1989), showed good comparability (less than a $20 \%$ difference for fluoride) with the known concentrations. Although the differences between this equipment and the set-up used by CELS are small they may be important enough to explain the CELS discrepancies.

\section{REFERENCES}

(1) R.W. Goles. August 1989. "Determination of Halogen Content in Glass for Assessment of Melter Decontamination Factors." HWVP-89IVJ0020500D. Pacific Northwest Laboratory, Richland, WA. 
WHC-SD-WM-ER-517

Revision 0

This page intentionally left blank. 


\section{DISTRIBUTION SHEET}

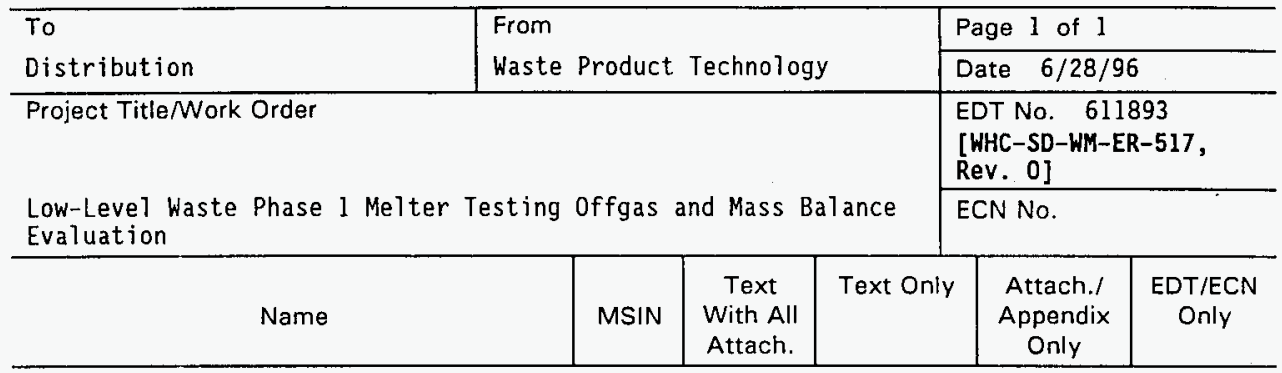

U.S. Department of Energy,

Richland Operations office

N. R. Brown

K6-51

S7 -53

$x$

K6-51

$\mathrm{K} 6-51$

R. A. Gilbert

S7 -53

L. A. Huffman

$X$

. E. Lamont

Pacific Northwest National Laboratory

$X$. Feng

C. J. Freeman

P. R. Hrma

J. M. Perez

M. R. Powel?

D. Vela

J. H. Westsik

G. A. Whyatt

K. D. Wiemers

Westinghouse Hanford Company

M. J. Bowman

K. C. Burgard

R. B. Calmus

D. W. Duncan

W. C. Eaton

J. S. Garfield

R. L. Gibby

D. W. Hendrickson

B. A. Higley

S. L. Lambert

A. F. Manue?

E. S. Mast

R. J. Murkowskj

S. R. Nelson

J. W. Shade

G. E. Stegen

D. J. Washenfelder

C. N. Wilson (3)

Central Files (Orig. +1)
P8-37

P7-41

P8-37

P7-41

P7-19

$\mathrm{K} 6-51$

K9-80

P7-19

K6-51 $x$

$x$

$X$
$X$

$\hat{x}$

$x$

$\hat{x}$
G1-25

H5-03

H5-27

B4-55

H5-27

H5-49

H5-27

ᄂ5-31

H5-27

H5-27

H5-49

S3-90

H5-03

B4-55

H5-27

H5-27

H5-27

H5-27

A3-88
X

$\underset{x}{x}$

$X$

$x$

$x$

$x$

$\mathrm{X}$

$x$

$\underset{x}{x}$

$x$

$X$
$X$

$x$

$x$

$x$

$x$ 